Florida International University FIU Digital Commons

\title{
The Discrimination and Association of Float Glass and The Quantitative Analysis of Liquids from Aerosols and Microdrops using Laser Induced Breakdown Spectroscopy
}

Erica M. Cahoon

Florida International University, ecaho001@fiu.edu

DOI: $10.25148 /$ etd.FI12050227

Follow this and additional works at: https://digitalcommons.fiu.edu/etd

\section{Recommended Citation}

Cahoon, Erica M., "The Discrimination and Association of Float Glass and The Quantitative Analysis of Liquids from Aerosols and Microdrops using Laser Induced Breakdown Spectroscopy" (2012). FIU Electronic Theses and Dissertations. 621.

https://digitalcommons.fiu.edu/etd/621 


\title{
FLORIDA INTERNATIONAL UNIVERSITY
}

Miami, Florida

\section{THE DISCRIMINATION AND ASSOCIATION OF FLOAT GLASS AND THE QUANTITATIVE ANALYSIS OF LIQUIDS FROM AEROSOLS AND MICRODROPS USING LASER INDUCED BREAKDOWN SPECTROSCOPY}

\author{
A dissertation submitted in partial fulfillment of \\ the requirements for the degree of \\ DOCTOR OF PHILOSOPHY \\ in \\ CHEMISTRY \\ by
}

Erica M. Cahoon 
To: Dean Kenneth G. Furton

College of Arts and Sciences

This dissertation, written by Erica M. Cahoon, and entitled The Discrimination and Association of Float Glass and The Quantitative Analysis of Liquids from Aerosols and Microdrops using Laser Induced Breakdown Spectroscopy, having been approved in respect to style and intellectual content, is referred to you for judgment.

We have read this dissertation and recommend that it be approved.

$\begin{array}{r}\hline \text { Yong Cai } \\ \hline \text { Surendra Saxena } \\ \hline \text { Rudolf Jaffe } \\ \hline \text { Kenneth Furton } \\ \hline \text { José Almirall, Major Professor }\end{array}$

Date of Defense: April 10, 2012

The dissertation of Erica M. Cahoon is approved.

Dean Kenneth G. Furton College of Arts and Sciences

Dean Lakshmi N. Reddi University Graduate School

Florida International University, 2012 
(C) Copyright 2012 by Erica M. Cahoon

All rights reserved. 


\section{DEDICATION}

This dissertation is dedicated to my family and friends. My mother, you demonstrated by example throughout my life that nothing is impossible and there is no incident too severe for which you allow the smile to be removed your face and strength from your heart. Without seeing this first-hand Mom, I don't believe I could have succeeded. You are my inspiration. My father, you have always been there for your little girl. Thank you for my second chance at life. I am blessed to have you both as parents. To all my friends who have walked beside me throughout the good and bad, I thank you all for your support. 


\section{ACKNOWLEDGMENTS}

The pages contained in this dissertation extend far further than a culmination of years of study. Throughout my graduate studies, I have been able to meet and interact with many generous and inspiring people. These pages also reflect those relationships. I value each contribution to my development as a scholar.

Dr. José Amirall, thank you for persevering with me as my mentor for the time it has taken me to accomplish this research and dissertation. I feel privileged to have been part of this program and worked underneath you. It is one of the most significant and formative experiences of my life.

I would like to express my gratitude to my graduate committee members: Dr. Yong Cai, Dr. Surendra Saxena, Dr. Rudolf Jaffe, and Dr. Kenneth Furton. Thank you for the generosity of your time and the expertise to further my studies.

I would like to acknowledge the Department of Chemistry and Biochemistry at FIU. To all the professors who challenged me to think, teach and explain. Thank you to the International Forensic Research Institute (IFRI), the Dean's Office, the Graduate Student Funding Committee and grants that allowed me funding to showcase my research and represent FIU at many scientific conferences. Thank you to the University Graduate School for awarding me the Dissertation Year Fellowship.

To my friends and loved ones, your support, love, kindness and forgiveness have kept me strong when I have been weak. Thank you to Adrian, Madelyn, Indira, Audrey, and Sarah.

To my colleagues, both past and present Dr. A team members, for sharing their thoughts and passion. A special thank you belongs with Sarah Jantzi, Emily Schenk, 
Tatiana Trejos, Howard Holness, Mimy Young, Wen Fan, Maria Perez, Steve Wise, Ben Naes and Monica Joshi. You have all enlightened my life. Please forgive me if I have failed to list anyone. 


\begin{abstract}
OF THE DISSERTATION
THE DISCRIMINATION AND ASSOCIATION OF FLOAT GLASS AND THE

QUANTITATIVE ANALYSIS OF LIQUIDS FROM AEROSOLS AND

MICRODROPS USING LASER INDUCED BREAKDOWN SPECTROSCOPY

by
\end{abstract}

Erica M. Cahoon

Florida International University, 2012

Miami, Florida

Professor José Almirall, Major Professor

Glass is a common form of trace evidence found at many scenes of crimes in the form of small fragments. These glass fragments can transfer to surrounding objects and/or persons and may provide forensic investigators valuable information to link a suspect to the scene of a crime. Since the elemental composition of different glass sources can be very similar, a highly discriminating technique is required to distinguish between fragments that have originated from different sources.

The research presented here demonstrates that Laser Induced Breakdown Spectroscopy (LIBS) is a viable analytical technique for the association and discrimination of glass fragments. The first part of this research describes the optimization of the LIBS experiments including the use of different laser wavelengths to investigate laser-material interaction. The use of a $266 \mathrm{~nm}$ excitation laser provided the best analytical figures of merit with minimal damage to the sample. The resulting analytical figures of merit are presented. The second part of this research evaluated the sensitivity of LIBS to associate or discriminate float glass samples originating from the 
same manufacturing plants and produced at approximately the same time period. Two different sample sets were analyzed ranging in manufacturing dates from days to years apart. Eighteen (18) atomic emission lines corresponding to the elements $\mathrm{Sr}, \mathrm{K}, \mathrm{Fe}, \mathrm{Ca}$, $\mathrm{Al}, \mathrm{Ba}, \mathrm{Na}, \mathrm{Mg}$ and $\mathrm{Ti}$, were chosen because of their detection above the method detection limits and for presenting differences between the samples. Ten elemental ratios producing the most discrimination were selected for each set. When all the ratios are combined in a comparison, $99 \%$ of the possible pairs were discriminated using the optimized LIBS method generating typical analytical precisions of $\sim 5 \%$ RSD.

The final study consisted of the development of a new approach for the use of LIBS as a quantitative analysis of ultra-low volume solution analysis using aerosols and microdrops. Laser induced breakdown spectroscopy demonstrated to be an effective technique for the analysis of as low as $90 \mathrm{pL}$ for microdrop LIBS with $1 \mathrm{pg}$ absolute LOD and $20 \mu \mathrm{L}$ for aerosol LIBS with an absolute LOD of $\sim 100 \mathrm{fg}$. 


\section{TABLE OF CONTENTS}

CHAPTER

PAGE

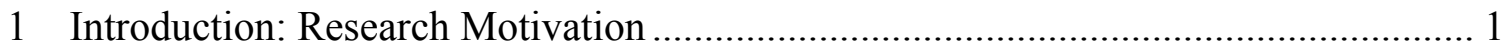

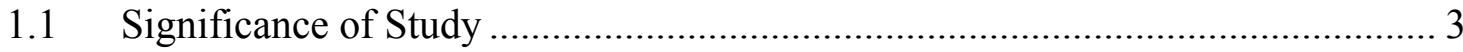

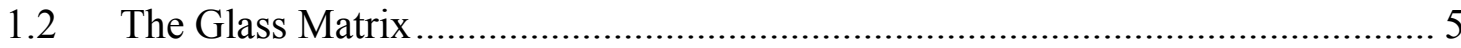

$1.3 \quad$ Elemental Analysis of Glass ......................................................................... 9

1.4 Sample Preparation of Solutions and Solid Samples for Chemical Analysis ... 15

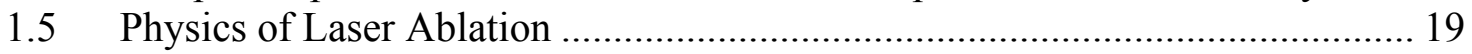

1.6 Introduction to Laser Induced Breakdown Spectroscopy …………………....... 22

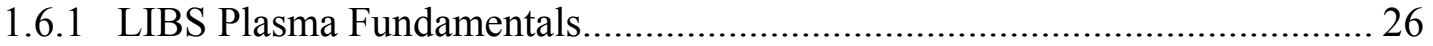

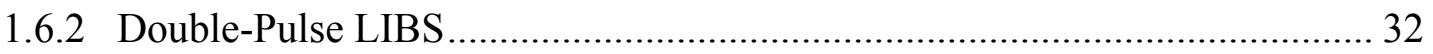

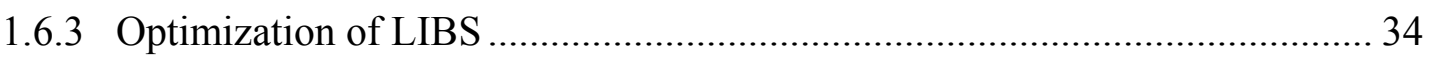

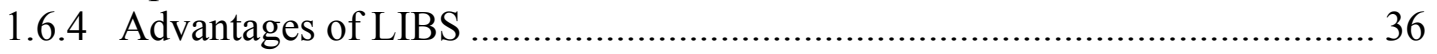

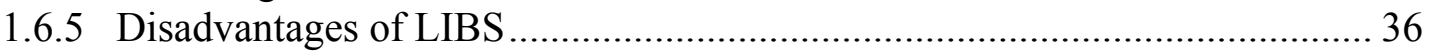

1.7 Principles of Laser Ablation Inductively Coupled Mass Spectrometry .............. 39

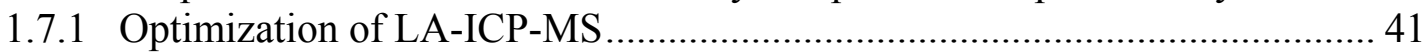

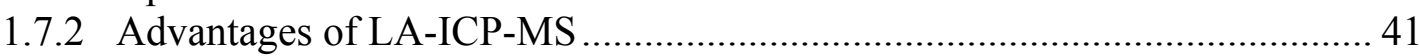

1.7.3 Disadvantages of LA-ICP-MS................................................................ 42

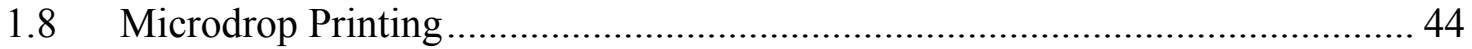

1.8.1 Piezoelectric Microdrop Printing................................................................ 46

1.8.2 Terminal Velocity of Microdrops ............................................................... 48

2 Wavelength Dependence of the Forensic Analysis of Glass by Laser Induced

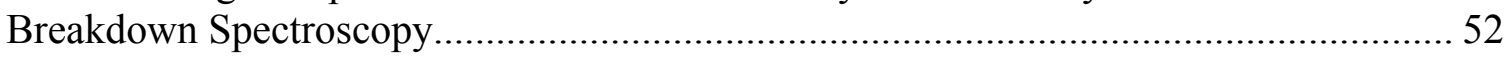

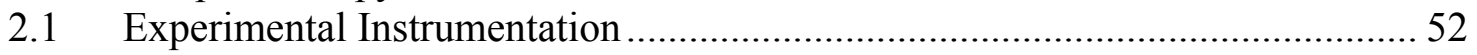

2.2 Effects of Laser Wavelength ................................................................ 57

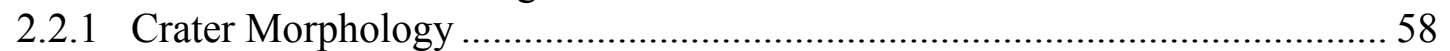

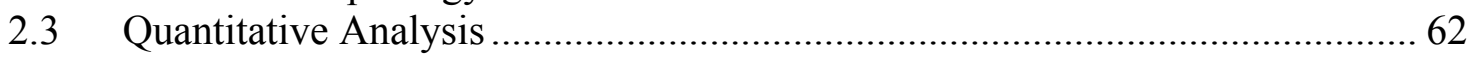

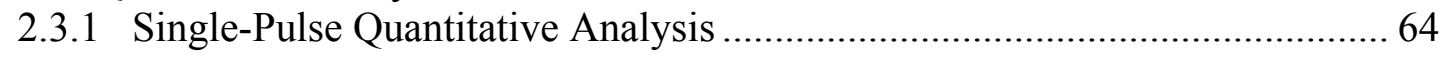

2.3.2 Double-Pulse Quantitative Analysis.............................................................. 68

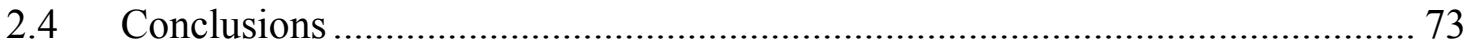

3 Discrimination and Association of Float Glass by Laser Induced Breakdown Spectroscopy from Single Manufacturing Plants ……………………......................... 75

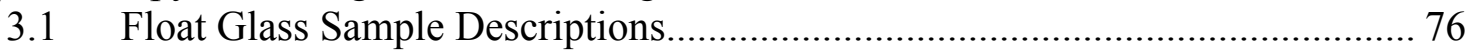

3.1.1 Sample Set 1: Cardinal Glass Industries...................................................... 76

3.1.2 Sample Set 2: Pilkington North America, Inc. …………………….............. 79

3.2 Experimental Instrumentation and Procedure .................................................. 80

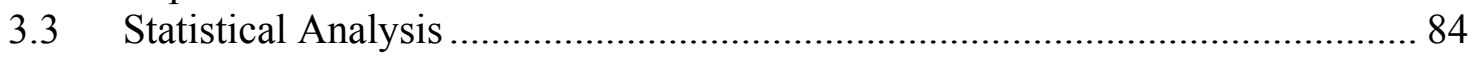

3.3.1 Discrimination Results LIBS: Cardinal Industries .......................................... 84

3.3.2 Discrimination Results LIBS: Pilkington North America, Inc....................... 87

3.4 Round Robin Glass Studies..................................................................... 93

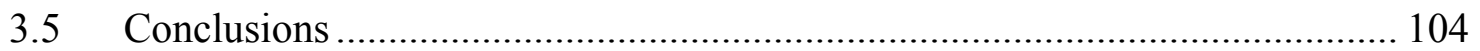


4 Laser Ablation Inductively Coupled Mass Spectrometry for the Discrimination and

Association of Float Glass from Single Manufacturing Plants...................................... 106

4.1 Glass Sample Set for LA-ICP-MS ………….......................................... 106

4.2 LA-ICP-MS Experimental Instrumentation for Glass Analysis ...................... 107

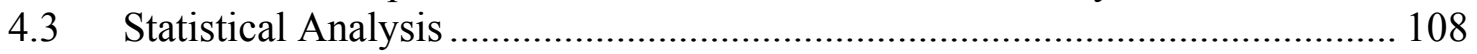

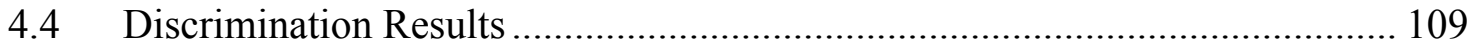

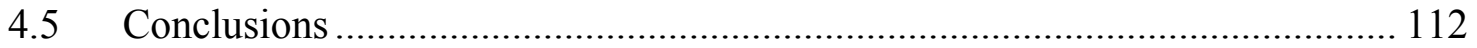

5 Quantitative Analysis of Liquids from Aerosols and Microdrops using Laser Induced Breakdown Spectroscopy ..................................................................................... 113

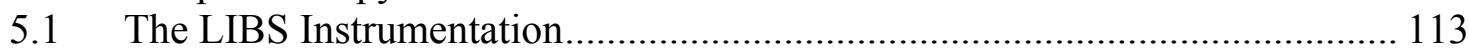

5.2 LIBS Optimization for Aerosol and Microdrop analysis ................................ 115

5.2 Aerosol LIBS Experimental Instrumentation................................................. 123

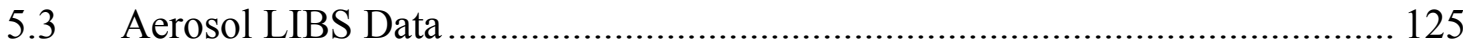

5.3.1 Aerosol LIBS: Single-pulse and Double-pulse quantitative analysis........... 125

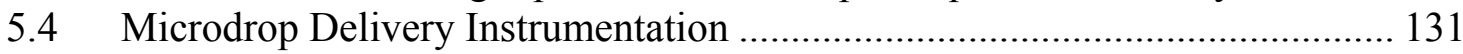

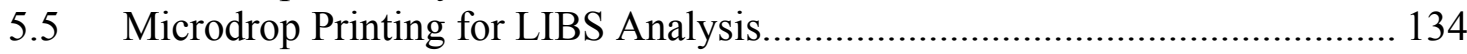

5.5.1 Microdrop Quantification on Aluminum Stub ………………………......... 134

5.5.2 Microdrop Mapping with LIBS ............................................................... 140

5.5.2 Microdrop Analysis with LIBS in Air ....................................................... 143

5.5.3 Microdrop Data Analysis...................................................................... 145

5.5.4 Single-Pulse and Double-Pulse Microdrop LIBS......................................... 146

5.6 Internal Standardization for Solution Analysis by LIBS ............................... 149

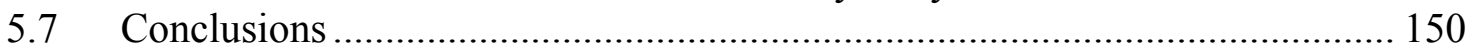

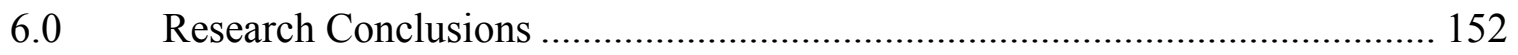

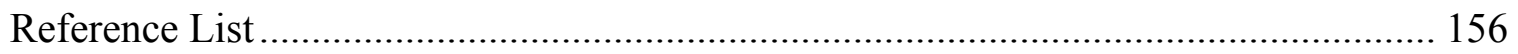

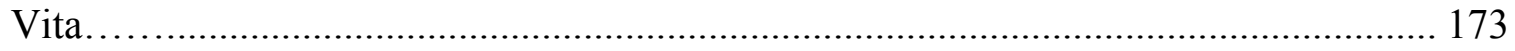




\section{LIST OF TABLES}

TABLE

PAGE

Table 1: Certified Concentration of Glass Standards $(\mu \mathrm{g} / \mathrm{g})$......................................64

Table 2: Figures of Merit for the Single-Pulse UV 266 nm (SP UV) ...........................68

Table 3: LIBS Figures of Merit for Single-Pulse, Double-Pulse Reheat, Double-Pulse

Prespark and Single-Pulse 1064 nm Laser. ........................................................... 72

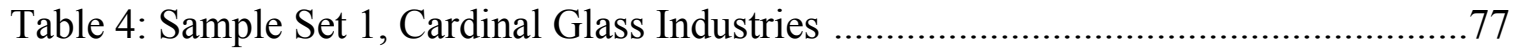

Table 5: Sample Set 2, Pilkington North America, Inc. ............................................79

Table 6: LIBS Discriminating Ratios and the Percent Discrimination for Sample Set 1,

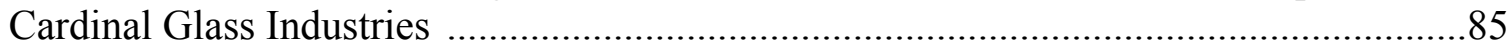

Table 7: Indistinguishable pairs by LIBS for Sample Set 1, Cardinal Glass Industries ...87

Table 8: LIBS Discriminating Ratios and the Percent Discrimination for Sample Set 2,

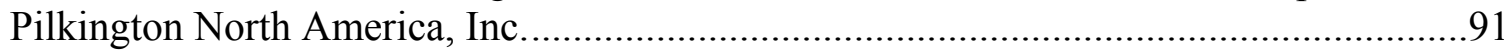

Table 9: Indistinguishable pairs by LIBS for Sample Set 2, Pilkington North America,

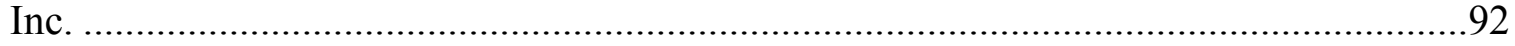

Table 10: LIBS Figures of Merit for CRM 1831 .......................................................92

Table 11: Description of Sample Set One for Round Robin Three ...............................96

Table 12: LIBS Discriminating Ratios and Results for Round Robin Three .................96

Table 13: Final Round Robin 3 Report, Comparison of K1 to Questioned Samples.......97

Table 14: Final Round Robin 3 Report, Comparison of K2 to Questioned Samples .......99

Table 15: Round Robin Three, Perrier Bottle Analyses ...........................................101

Table 16: LA-ICP-MS Discriminating Isotopes and Percent Discrimination for Pilkington America Inc.

Table 17: Excel Output for T-test Assuming Unequal Variances for Duplicate and Sample Pairs Manufactured Two Days Apart .........................................................111

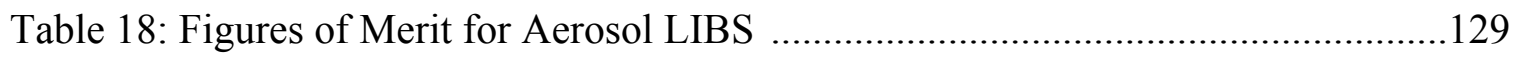


Table 19: Figures of Merit for Microdrop LIBS in Air .148 


\section{LIST OF FIGURES}

FIGURES

PAGE

Figure 1: The float glass furnace at Pilkington North American Inc., Stockton, CA ........6

Figure 2: Float glass being pulled onto rollers at Pilkington North American Inc., Stockton, CA

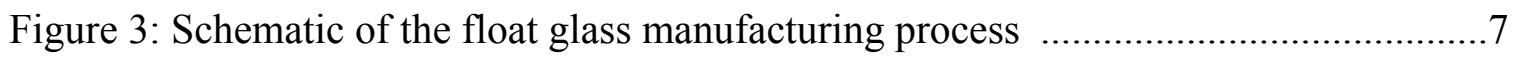

Figure 4: The time scale of events from laser pulse to particle ejection ........................20

Figure 5: Single-pulse and double-pulse LIBS schematic

Figure 6: The timescale from laser pulse to spectral emission. Continuum decay time delay, $t_{d}$, represents the time before detector opening and $t_{b}$ represents the integration time

Figure 7: Shadowgraph demonstrating shockwave growth from 880 ns to $1.15 \mu \mathrm{s}$

Figure 8: Schematic of the different double-pulse LIBS configurations: a) collinear, b) reheating orthogonal and c) prespark orthogonal

Figure 9: Schematic of ICP-MS instrument .39

Figure 10: Piezoelectric waveform for inkjet printing .48

Figure 11: Diagram of stokes sphere forces .50

Figure 12: Lens-to-sample distance optimization for LIBS glass analysis for background subtracted intensity or Lorentzian fit peak areas for a) Sr I $460.7 \mathrm{~nm}$ or b) Al I $394.4 \mathrm{~nm}$

Figure 13: Precision variation with laser energy on glass using $1064 \mathrm{~nm}$ laser .54

Figure 14: Optimized double-pulse LIBS prespark interpulse delay .55

Figure 15: Optimized double-pulse LIBS reheat interpulse delay .56

Figure 16: The damage caused to glass using IR $1064 \mathrm{~nm}$ laser .59

Figure 17: a) Crater formed using a $266 \mathrm{~nm}$ laser on NIST glass standard 1831 b) Crater formed using a $1064 \mathrm{~nm}$ laser on NIST glass standard 1831 .60 
Figure 18: Spectral emission lines for a) Ba II $493.4 \mathrm{~nm}$ (32 ppm), b) K I 766.5 nm (2738 ppm), and c) Sr II $421.5 \mathrm{~nm}(89 \mathrm{ppm})$.

Figure 19: a) Single-pulse $1064 \mathrm{~nm}$ calibration curves for K I $766.5 \mathrm{~nm}$; b) Single-pulse $266 \mathrm{~nm}$ calibration curve for K I $766.5 \mathrm{~nm}$; c) Single-pulse $1064 \mathrm{~nm}$ calibration curves for Sr II 407.7; d) Single-pulse $266 \mathrm{~nm}$ calibration curve for Sr II 407.7; e) Single-pulse $1064 \mathrm{~nm}$ calibration curves for Ba II 493.3; f) Single-pulse $266 \mathrm{~nm}$ calibration curve for Ba II $493.3 \mathrm{~nm}$

Figure 20: Calibration curves for double-pulse plasma reheat with $266 \mathrm{~nm}$ ablation for a) K I $766.5 \mathrm{~nm}$ and b) Ba II $493.4 \mathrm{~nm}$ .69

Figure 21: Calibration curves for double-pulse IR pre-spark with $266 \mathrm{~nm}$ ablation for a) K I $766.5 \mathrm{~nm}$ and b) Ti II $336.1 \mathrm{~nm}$ .71

Figure 22: LIBS experimental setup schematic for forensic glass discrimination .81

Figure 23: LIBS spectrum for CRM 1831 float glass. .82

Figure 24: The indistinguishable pairs by ANOVA pairwise comparison using LIBS for sample set 1 , Cardinal Glass Industries .86

Figure 25: LIBS signal illustrating the high to low Fe transition at Pilkington North America Inc. .88

Figure 26: Correlation between LIBS and LA-ICP-MS for the Fe transition at Pilkington North America, Inc. .89

Figure 27: The elemental profile of Fe for sample set 2 by LIBS and LA-ICP-MS ........90

Figure 28: Sample Set 2, Perrier bottle, for round robin three ………………………....100

Figure 29: Glass fragments provided for round robin four 102

Figure 30: Schematic of a) Microdrop LIBS in air or b) Aerosol LIBS experimental setup

Figure 31: Optimization of gate delay for a) Aerosol and b) Microdrop LIBS in air .....116

Figure 32: Optimization of integration time for double-pulse microdrop LIBS .117

Figure 33: Collinear interpulse delay optimization in aerosol LIBS for Sr II $407.7 \mathrm{~nm} .119$

Figure 34: Collinear interpulse delay optimization in microdrop LIBS for Sr II $421.5 \mathrm{~nm}$, Mg II $280.2 \mathrm{~nm}$, Ba II $455.5 \mathrm{~nm}$ and Ca II $396.8 \mathrm{~nm}$ .120 
Figure 35: Optimized argon flow rate for aerosol LIBS for a) Mg II 279.5 and b) $\mathrm{Sr} \mathrm{II}$ $407.7 \mathrm{~nm}$

Figure 36: Experimental aerosol LIBS setup ...........................................................123

Figure 37: Aerosol LIBS effluent exiting the orifice into LIBS plasma .........................125

Figure 38: Single-pulse aerosol LIBS spectra for concentrations ranging from 0-250 ppm

Figure 39: Double-pulse aerosol LIBS spectra of Sr II $407.7 \mathrm{~nm}$ emission line at a concentration of $1 \mathrm{ppm}$ and blank solution

Figure 40: Aerosol LIBS calibration curves for a) Mg II 279.5 and $280.2 \mathrm{~nm}$ b) Ba II 493.4 and $455.5 \mathrm{~nm}$ emission lines 128

Figure 41: JetDrive III experimental setup and controller .132

Figure 42: Jetlab 4 a) Microdrop System and b) viewing optics on Al stub substrate ...132

Figure 43: Printhead used for microdrop delivery .133

Figure 44: Crater on Al stub pre-LIBS analysis a) $200 \mu \mathrm{m}$ crater on Al stub for microdrop deposition b) Al stub crater with a depth of $15 \mu \mathrm{m}$ for microdrop deposition 135

Figure 45: Linear relationship of Sr mass ablated per laser pulse .136

Figure 46: Crater depth on Al stub post LIBS analysis 137

Figure 47: Al stub spectrum with $150 \mathrm{pg}$ of $\mathrm{Sr}$ deposited into crater 138

Figure 48: Calibration curves for microdrop LIBS on aluminum stub for a) Sr II $407.7 \mathrm{~nm}$ and b) Ba II $614.2 \mathrm{~nm}$

Figure 49: Jetlab 4 pattern printed for LIBS elemental mapping analysis 141

Figure 50: a) aluminum stub with printed pattern; b) aluminum stub after LIBS elemental mapping analysis 141

Figure 51: Two-dimensional plot of LIBS elemental mapping of $\mathrm{Sr}$ 142

Figure 52: Three-dimensional plot illustrating $\mathrm{Sr}$ concentration with LIBS elemental mapping 142

Figure 53: Experimental LIBS setup for microdrop LIBS in air .144 
Figure 54: a) $90 \mathrm{pL}$ drop ejected from the orifice of the printhead; b) The microdrop in the LIBS plasma being atomized and ionized..............................................................145

Figure 55: LIBS spectra of a microdrop double-pulse and single-pulse spectra for $\mathrm{Sr}$ peaks $407.7 \mathrm{~nm}, 421.5 \mathrm{~nm}$ and $460.7 \mathrm{~nm}$ with 90 picograms of $\mathrm{Sr}$ 146 


\section{LIST OF ABBREVIATIONS}

LIBS Laser Induced Breakdown Spectroscopy

SP Single-pulse

DP Double-pulse

ICP-MS Inductively Coupled Plasma Mass Spectrometry

LA Laser Ablation

LA-ICP-MS Laser Ablation Inductively Coupled Plasma Mass Spectrometry

$\mu \mathrm{XRF} \quad$ Micro X-ray Fluorescence

SEM-EDS Scanning Electron Microscopy Energy Dispersive Spectroscopy

WDS Wavelength Dispersive Spectroscopy

AAS Atomic Absorption Spectroscopy

NAA Neutron Activation Analysis

NIST National Institute of Standards and Technology

ASTM American Society of Testing and Materials

FIU Florida International University

CRM Certified Reference Material

Nd:YAG Neodymium: Yttrium Aluminum Garnet

FWHM Full Width Half Maximum

LTE Local Thermodynamic Equilibrium

ppm Parts Per Million

UV Ultraviolet

IR Infrared

SD Standard Deviation 


$\begin{array}{ll}\text { RSD } & \text { Relative Standard Deviation } \\ \text { LTSD } & \text { Lens-To-Sample Distance } \\ \text { CCD } & \text { Charge Coupled Device } \\ \text { LOD } & \text { Limit of Detection }\end{array}$

xviii 


\section{Introduction: Research Motivation}

Glass is a commonly encountered type of trace evidence found at many crime scenes. Glass fragments may provide the forensic investigator with valuable information. The fragments are often produced by breaking a window during a forced entry or as a result of the high forces to vehicle windows during a hit-and-run accident or other collisions.

These glass fragments may then be transferred to all surrounding objects and persons and may provide forensic investigators valuable information of association to link a suspect to the scene of a crime. If the glass was broken during a crime act, these glass fragments could potentially be used as evidence, associating an individual to the event.

Since the elemental profile of glass can be very similar, a highly discriminating technique is required to distinguish between fragments that have originated from different sources. Historically, the forensic analyses of glass fragments have been based on the comparison of color, density, thickness, transparency and refractive index. As a result of the lack of discrimination power of these analytical techniques, a need developed for more sensitive, complementary instrumentation for the forensic analysis of glass fragments. For instance, refractive index was the method of choice for many forensic laboratories, however, glass manufacturers now target specific optical properties in glass, creating a narrow range of refractive indices ${ }^{1}$. As a result, elemental analysis has been suggested as an additional technique ${ }^{2,3}$. With use of more sensitive analytical techniques, the forensic analyst may associate or discriminate, with a high degree of certainty, between the glass fragments in question to those of known origin. These associations may establish a connection between the suspect and scene of the crime. 
Varying concentrations in float glass may allow glass to be chemically characterized to its source. If the variation in elemental composition of float glass is greater between sources than within sources, this variation allows for the forensic discrimination of glass. Certain elements are controlled in the glass manufacturing process to obtain specific properties for the end-use product. The major elements which are controlled may provide discrimination ${ }^{4}$. However, it is predominantly the minor, trace and the impurities that provide the forensic investigator the ability to discriminate or associate glass samples ${ }^{5}$.

Although LIBS is more commonly used for analysis of solid matrices, solution analysis is investigated in this research. Solutions provide the analyst a homogeneous sample that can incorporate internal standards and are easily matrix-matched. Most sampling volume requirements are approximately a milliliter or more for the wellestablished techniques of inductively coupled plasma mass spectrometry (ICP-MS) or ICP-optical emission spectrometry (ICP-OES) ${ }^{6,7}$. Very small volumes, i.e., picoliters to microliters, present a considerable challenge when measuring very low concentrations of trace metals.

My research describes the development and validation of the use of Laser Induced Breakdown Spectroscopy as an analytical technique for the association and discrimination of forensic glass and for ultra-low volume analysis of aerosols and, for the first time, microdrops delivered in an ambient atmosphere. 


\subsection{Significance of Study}

Laser induced breakdown spectroscopy will be presented as an analytical tool for the forensic association and discrimination of float glass by elemental profiling. Secondly, LIBS will be presented as a tool for the quantitative analysis of small volumes from aerosols and, for the first time, microdrops.

Float glass is a commonly encountered type of evidence for the forensic investigator. The similar elemental composition of float glass requires techniques with high analytical sensitivity and reproducibility so the forensic analyst may achieve accuracy and discrimination. The use of elemental analysis for the forensic glass comparisons is not a recent development. It dates back to as early as 1973 with the use of neutron activation analysis (NAA) ${ }^{8}$ to distinguish glass from different sources. Currently, forensic laboratories perform elemental profiling of glass predominantly using micro x-ray fluorescence ( $\mu \mathrm{XRF),} \mathrm{ICP-MS} \mathrm{and} \mathrm{laser} \mathrm{ablation-ICP-MS} \mathrm{(LA-ICP-MS).}$

The mature techniques of $\mu \mathrm{XRF}$, ICP-MS and LA-ICP-MS are advantageous; however each method has limitations. Minor and trace elements present in low concentrations are problematic for $\mu \mathrm{XRF}$ as they lie below the analytical limits of detection (LOD). Milligrams of sample are required for the necessary repeated analysis with ICP-MS ${ }^{7,9-11}$, which may be unmanageable when only small fragments are transferred during a crime. The invention of laser ablation for sample introduction into the ICP-MS has simplified the analysis of solids, such as float glass, and is now regarded as the "gold standard" for discrimination between similar elemental profiles of glass samples originating from different sources, or to determine the association from the same source $^{3,12-15}$. Despite the excellent analytical performance of LA-ICP-MS, this 
instrumentation is complex, expensive and is not available for many forensic laboratories. Without forensic laboratories having access to sensitive analytical techniques, a need is created to develop a less expensive and robust method that can be used for the chemical characterization of trace evidence, such as float glass, that is commonly encountered in the forensic laboratory.

The studies that are presented throughout this manuscript demonstrate the utility of LIBS as an alternative analytical technique for the forensic analysis of glass. The research in the chapters to follow describes method development and validation for LIBS as an analytical technique for the forensic analysis of glass. The optimization of laser energy, gate delay, integration times and laser irradiance to improve crater morphology, all for improving reproducibility, limits of detection, precision and accuracy are discussed. The discrimination capabilities of LIBS will be evaluated by two float glass sample sets: 1) consisting of 49 colorless float glass samples manufactured in a single plant from May 1997 to September 2001 and 2) consisting of 27 colorless float glass samples collected from September 2008 to April 2010 from a different single manufacturing plant, both sets range in manufacturing date from days to years apart. On the basis of LIBS still being a forthcoming technique, the results produced are compared to LA-ICP-MS. LA-ICP-MS is also evaluated on the percent discrimination achieved for float glass sample set two.

The final study consists of developing an analytical method to demonstrate the analytical capabilities of LIBS as a technique for the quantification of low volume solution analysis with aerosols and microdrops. Employing LIBS as an analytical technique for solutions, eliminates certain disadvantages observed with LIBS liquid 
analyses, such as splashing, while also eliminating the greater volume requirements needed for solution sampling by techniques such as ICP-MS or ICP-OES.

\subsection{The Glass Matrix}

The American Society of Testing and Materials (ASTM) defines glass “....as an inorganic product of fusion which has been cooled to a rigid condition with crystallization..."16. Common raw materials for float glass manufacture consist of silica $\left(\mathrm{SiO}_{2}\right)$ that is responsible for the high transition temperature of glass and sodium carbonate which lowers the transition temperature. Other ingredients added are dolomite, calcium carbonate, magnesium oxide, sodium oxide and aluminum oxide, as they adjust chemical and physical properties in glass ${ }^{17}$.

Glass is usually classified into three groups by its chemical composition: 1) soda lime glass, 2) lead glass and 3) borosilicate glass. These three types account for $95 \%$ of all glass, with soda lime glass being $72 \%$ silica, it constitutes for approximately $90 \%$ of manufactured glass ${ }^{18}$. Bottles, jars, architectural and automobile glass are examples of soda-lime glass. The many applications of soda-lime glass is a result of its physical and chemical properties, with light transmission being the most significant ${ }^{19}$. The smooth, non-porous surface allows for bottles to be easily cleaned, to be filled with beverages with no affect to flavor and with no harmful substances. Lead glass, the second group, is produced by replacing much of the lime with lead oxide. Lead glass exhibits high refractive index, which are used most as decorative glass, i.e., vases or bowls. Borosilicate, the third group, is resistant to chemical corrosion and temperature change as 
a result of the high $\mathrm{SiO}_{2}$ in the glass ${ }^{19}$. Borosilicate glass is commonly used in the chemical laboratory.

The manufacturing of float glass consists of mixing the batch (raw material) with crushed cullet (recycled glass). The melting furnace is made of refractory bricks and heated to approximately $2900^{\circ} \mathrm{F}$. The furnace has a capacity to contain as much as 2000 $\mathrm{kg}$ of raw materials, see Figure 1. The glass then leaves the furnace at a temperature of approximately $1900^{\circ} \mathrm{F}$ and is fed into a bath of molten tin.

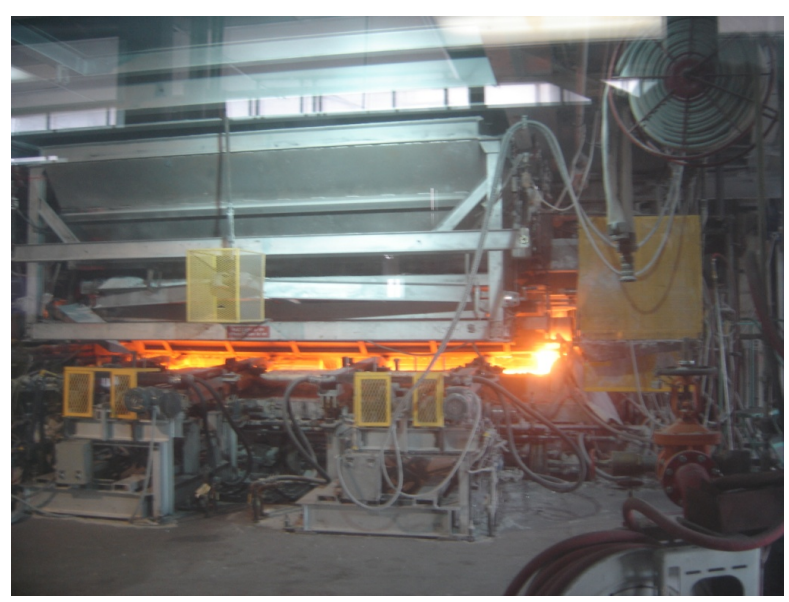

Figure 1. The float glass furnace at Pilkington North American Inc., Stockton, CA

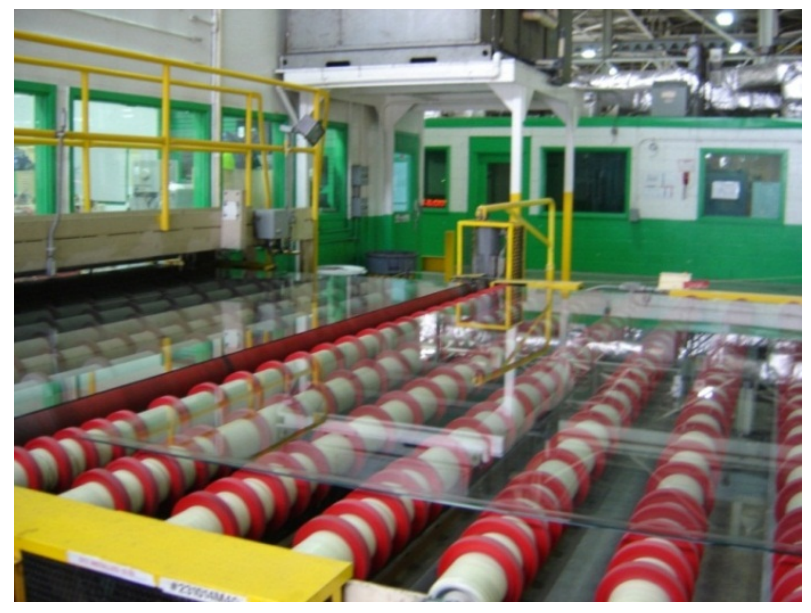

Figure 2. Float glass being pulled onto rollers at Pilkington North American Inc., Stockton, CA 
Tin is appropriate for the float glass process as a result of the high specific gravity of tin and its immiscibility into the molten glass. The tin bath is kept in a controlled atmosphere of hydrogen and nitrogen to prevent oxidation. The glass spreads out flat and smooth on the molten tin. The thickness of the glass is controlled by the pull of a ribbon, see Figure 2. The two photographs above, Figure 1 and 2 were provided by my mentor, Dr. Almirall.

The temperature is gradually decreased to approximately $1000^{\circ} \mathrm{F}$ in the annealing lehr, where the glass sheet can be placed on the rollers. The glass in then cut to a predetermined sizes ${ }^{18,20,21}$. Figure 3 illustrates the different processes for the manufacture of float glass ${ }^{21}$. The standard specifications for float glass have been established in method ASTM C 1036 Standard Specification for Flat Glass.

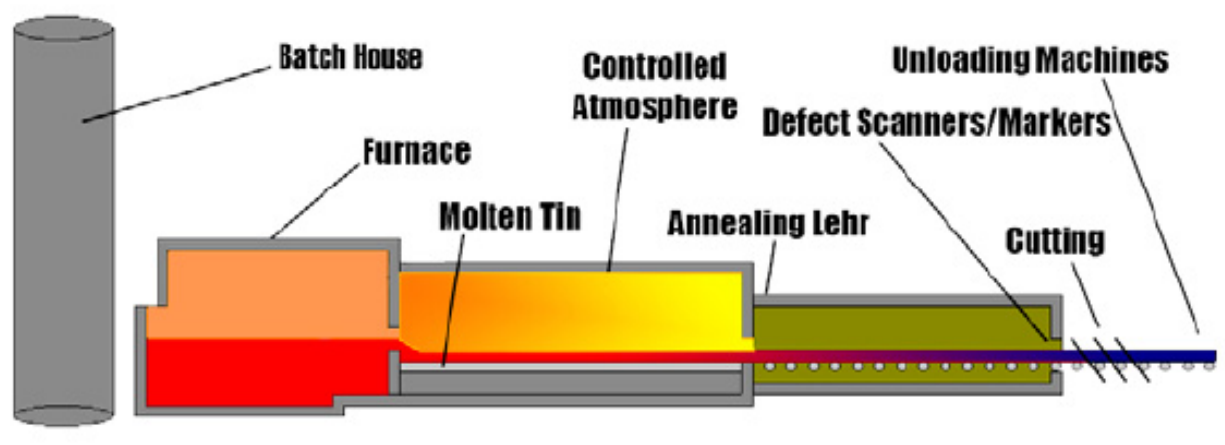

Figure 3. Schematic of the float glass manufacturing process ${ }^{21}$

There are approximately 40 float glass plants in the United States corresponding to eight manufacturers: Cardinal Glass Industries, Guardian Industries Corporation, AFG Industries, Inc., ACH Float Glass Operations, Pilkington North American, Inc., PPG Industries, Inc., Saint-Gobain Glass, and Vitro America, Inc. These plants are designed to operate seven days a week, 365 days a year for ten years or more, typically producing 
between $300-600$ tons of glass per day ${ }^{21}$. The lifetime of the furnace is approximately 10 years as a result of the corrosion of the refractory bricks ${ }^{19}$. It is the process of this corrosion that causes differences in the trace elemental composition. It is these trace elements that provide the greatest discrimination between different sources and dates of manufacture between glasses.

Float glass manufacturing plants, Cardinal Glass Industries and Pilkington North America, Inc., provided float glass samples for this research. 


\subsection{Elemental Analysis of Glass}

Numerous elemental analysis techniques have been employed for the discrimination and chemical characterization of glass. These techniques include spark source mass spectrometry ${ }^{22}$, atomic emission spectroscopy (AES) ${ }^{23}$, atomic absorption spectroscopy (AAS) ${ }^{2}$, x-ray fluorescence $(\mathrm{XRF})^{2,23,24}$, neutron activation analysis (NAA) $)^{8}$, scanning electron microscopy (SEM) coupled to both energy dispersive spectroscopy (EDS) and wavelength dispersive spectroscopy (WDS) ${ }^{24-26}$, particle induced x-ray emission (PIXE) ${ }^{25}$, inductively coupled plasma atomic emission spectrometry (ICPAES) $)^{2,23,25}$, inductively coupled plasma mass spectrometry (ICP-MS) ${ }^{11,23,27}$, laser ablation inductively coupled plasma mass optical emission spectrometry (LA-ICPOES) ${ }^{27}$, laser ablation-ICP-MS (LA-ICP-MS) $)^{3,12-15}$ and LIBS ${ }^{3,13,28-31}$.

The potential to discriminate glass was documented with NAA, as early as $1973^{8}$. Samples analyzed by NAA are bombarded with neutrons, causing the elements to form radioisotopes. Different radioisotopes have different half-lives and these radioactive decay paths for each element are well known. The radioactive emissions are counted, providing the analyst with the elemental composition. Coleman et al. ${ }^{8}$ analyzed 25 elements from window glass originating from England and Wales using neutron activation analysis. The authors demonstrated that the frequency distributions of the elemental concentrations could be used for the forensic comparison of glass.

Scanning electron microscopy uses a focused beam of high-energy electrons causing the emission of X-rays. X-ray fluorescence spectrometry relies upon the measurement of characteristic X-rays produced by an X-ray source. X-ray radiation is produced by when an inner electron of the atom is displaced by either the high-energy 
electron beam or X-ray beam. Electrons in higher energy states relax to fill the lower energy states. The energy difference between the two electronic states is given off as Xrays. The intensities of these X-rays are proportional to the elemental concentration, providing qualitative and quantitative information. For the discrimination among different glass sources, SEM and XRF report the use of elemental ratios for the intensities in glass ${ }^{24,26}$. Dudley et al. in 1980 used XRF and 10 elemental ratios with Ca for determining the elemental concentrations in 50 pairs of window and non-window glasses having indistinguishable refractive indices. The elemental ratios discriminated $95 \%$ of the glass samples. Ryland correctly classified $93 \%$ of sheet and container glass using a $\mu \mathrm{XRF}$ comparing the $\mathrm{Ca} / \mathrm{Fe}$ ratio $^{26}$. He reported that a high $\mathrm{Ca} / \mathrm{Fe}$ ratio is indicative of container glass or tableware while a low $\mathrm{Ca} / \mathrm{Fe}$ ratio is representative of sheet glass. Advantages of $\mathrm{XRF}$ are that it is a non-destructive technique with little interferences from spectral lines. Disadvantages are high limits of detection $(\sim 100 \mathrm{ppm})$ and glass fragments are often irregularly shaped, affecting take-off angles and causing poor precision and accuracy.

The inductively coupled plasma may either be coupled with an optical emission spectrometer or a mass spectrometer. The inductively coupled plasma is a radiofrequency-induced plasma from an induction coil to produce a magnetic field. When coupled to an optical emission spectrometer, the light is separated according to its wavelength and the intensities are characteristic of the elemental concentration. The mass spectrometer separates ions according to their mass-to-charge ratio. These detectors are very sensitive while also quantitative. Both these methods require the samples to be in solution. The dissolution of glass requires an elaborate method. The ASTM provides a protocol for the dissolution and analysis of glass by ICP-OES ${ }^{32}$. ICP-OES is a 
destructive technique where the glass fragments need to be crushed and digested in a combination of $\mathrm{HF}, \mathrm{HNO}_{3}$ and $\mathrm{HCl}$. The glass and acid mixture are then heated till dissolution of the glass.

Hickman was the first to propose a classification procedure for container, sheet, tableware or headlamp glass using ICP-OES ${ }^{33,34}$. He analyzed manganese (Mn), iron $(\mathrm{Fe})$, magnesium $(\mathrm{Mg})$, aluminum $(\mathrm{Al})$ and barium $(\mathrm{Ba})$ with refractive index and was able to discriminate $91 \%$ of 349 glass samples. Hickman later expanded the elemental menu to include; strontium (Sr), Fe, arsenic (As), cerium (Ce), cobalt (Co), cesium (Cs), lithium $(\mathrm{Li})$, rubidium $(\mathrm{Rb})$, antimony $(\mathrm{Sb})$ and lead $(\mathrm{Pb})$, to provide higher discrimination $^{34-36}$. Koons et al. compared 182 sheet and container glasses by ICP-OES ${ }^{9}$ using $\mathrm{Al}, \mathrm{Ba}, \mathrm{Mg}, \mathrm{Fe}, \mathrm{Sr}, \mathrm{Mn}, \mathrm{Ca}, \mathrm{Na}$ and $\mathrm{Ti}$. He classified all samples correctly except for two container glasses from the same manufacturing plant classified as sheet glass and two sheet glasses classified as container glass. Koons et al. classified 180 of the 184 samples using principal component analysis (PCA) and cluster analysis ${ }^{9}$. This study concluded that elemental composition can classify glass samples from the same manufacturer, thereby suggesting that the elemental profile can provide good evidence of an association between the manufacturer and the glass fragment. Parouchais et al. ${ }^{37}$ used ICP-MS to analyze 62 elements within glass. To decrease physical, spectral and chemical interferences that may arise from the dissolution of glass, the authors reported using elemental ratios to discriminate between samples found indistinguishable by refractive index. Suzuki et al. ${ }^{10}$ used pairwise comparison of the elements $\mathrm{Co}, \mathrm{Rb}, \mathrm{Sr}, \mathrm{Zr}, \mathrm{Ba}, \mathrm{La}$ and Ce to discriminate 22 windshield float glass samples. The authors reported $100 \%$ discrimination using elemental composition and $94 \%$ discrimination with refractive 
index. With a change in the elemental menu, $\mathrm{Zr}, \mathrm{Ba}, \mathrm{Sb}, \mathrm{Sr}, \mathrm{Hf}, \mathrm{As}$, Mo and $\mathrm{Pb}$, Suzuki et $a l .{ }^{38}$ were able to distinguish between all 17 samples (138 pairs) of headlight glass with ICP-MS and only 19 pairs with refractive index.

In 1985, Gray ${ }^{39}$ developed laser ablation (LA) as a new sample introduction system for ICP-MS. This introduction generates particles when the laser interacts with the solid sample. These particles are then swept into the ICP-MS with a carrier gas. Laser ablation eliminated the laborious and time consuming solid sample preparation, while providing the same sensitive technique. Laser ablation brought about several advantages; practically no sample preparation, no contamination from chemicals, less interferences as a result of no solvents and spatial resolution. A more in-depth explanation of LA-ICP-MS will be provided in the next section.

To validate LA-ICP-MS, it was compared to the mature technique of ICP-MS. Individual studies were done demonstrating that LA-ICP-MS provided equally as good figure of merits (precision, bias, and limit of detection) ${ }^{12,40,41}$. Trejos et al. ${ }^{41}$ conducted micro-homogeneity study to evaluate the elemental composition of glass samples found at crime scenes. The results demonstrated that float glass is homogeneous at the microanalysis level, allowing LA-ICP-MS to be viable technique for the forensic analysis of float glass. Inherent heterogeneity was observed with headlamps and container glass. The authors recommended sampling at many different locations to correctly characterize the elemental profile. An inter-laboratory cross validation study was conducted to verify different laser ablation systems with different ICP-MS instrumentation. The objective was to compare inter-laboratory results for similarity in efforts to improve analytical 
protocol $^{14}$. It was determined that many different laboratories were able to produce standard deviation of less than $10 \%$ for most all elements.

Laser induced breakdown spectroscopy is an emerging atomic emission technique for the forensic analysis of glass. The current history of LIBS for the elemental analysis of glass is limited, as no standard protocols currently exist. The LIBS research conducted to date has been successful in the forensic analysis of glass ${ }^{3,13,28,42}$. Naes et al. ${ }^{3}$ demonstrated that LIBS produces the same 99\% discrimination, with no false exclusions, as $\mu$ XRF and LA-ICP-MS with a different element menu of automobile float glass ${ }^{3}$. Naes et al. found the 10 most discriminating elemental ratios to be $\mathrm{Al} / \mathrm{Na}, \mathrm{K} / \mathrm{Ca}, \mathrm{Al} / \mathrm{Fe}$, $\mathrm{Fe} / \mathrm{K}, \mathrm{Ca} / \mathrm{K}, \mathrm{Fe} / \mathrm{Al}, \mathrm{K} / \mathrm{Ca}, \mathrm{Al} / \mathrm{Sr}, \mathrm{Sr} / \mathrm{K}$ and $\mathrm{Na} / \mathrm{K}$. Bridge et al. achieved an $83 \%$ and $74 \%$ discrimination of automobile float glass with LIBS, however when combined with refractive index measurements, the discrimination increased to $99 \%$ discrimination for both sample sets, but these authors did not conduct type I and type II error studies ${ }^{13,42}$. The precision obtained from both authors were in agreement, Naes et al. state the precision is less than $10 \% \mathrm{RSD}$ and Bridge et al. state an average precision of $7 \% \mathrm{RSD}$.

LIBS offers a sensitive and fast approach to elemental analysis and permits small sample size with good precision, similar to LA-ICP-MS. LIBS is fairly inexpensive when compared to the more established techniques of LA-ICP-MS, LA-ICP-OES and $\mu$ XRF. LIBS is less complex, generates data virtually instantaneously, has the capability to be portable, while providing high sample throughput.

The present section summarizes the advancements that have been made for the elemental analysis of glass by many different techniques. The recent developments in LIBS, has shown it to be a promising technique for the forensic analysis of glass. The 
research studies to follow will demonstrate that LIBS is indeed a viable alternative to the expensive instrumentation of LA-ICP-MS for the forensic analysis of glass and should be given consideration in the forensic laboratory. 


\subsection{Sample Preparation of Solutions and Solid Samples for Chemical Analysis}

Laser Induced Breakdown Spectroscopy is predominantly a solid sampling technique that has been demonstrated to compete favorably with $\mu$ XRF-EDS and LA-ICP-MS ${ }^{43,44}$ for the elemental analysis of some solid matrices. Because of its multi-elemental detection, non-destructive nature, minimal sample preparation and in-situ analysis, LIBS has become a very popular analytical technique offering simplicity of operation and data analysis as attractive features.

Analytical chemists use atomic spectroscopy tools for both solution and solid analysis, and laser-based solid sampling tools have been coupled to ICP plasmas. Techniques based on atomic spectroscopy are important in trace analysis of solutions but many solution techniques suffer from labor intensive and time-consuming sample preparation. Solutions provide homogeneous samples that can incorporate internal standards and external calibration methods can be easily developed but require sample preparation (sample dissolution) when the sample is in solid form. Direct solid sampling may also require sample preparation, primarily to homogenize the sample prior to analysis by pelletizing a powder sample, for example. The sample is milled into a homogeneous powder and then pressed into a pellet ideally producing a flat, homogeneous surface. In an effort to improve precision and decrease elemental fractionation, particle size effects have been investigated. It has been reported that, smaller particle sizes produce enhanced ionization in the plasma in the LA-ICP-MS experiment ${ }^{45-47}$. Arroyo et al. found that reducing the particle size to less than $1 \mu \mathrm{m}$ allowed for representative sampling and improved precision on soil samples ${ }^{47}$. 
Homogeneity is essential in laser micro-sampling techniques, i.e., LIBS and LAICP-MS, where only tens to hundreds of nanograms to micrograms of material are removed for analysis. Many factors pose challenges during solid sampling, such as sample size, shape, heterogeneity and the lack of matrix-matched standards. Without adequate sample size, one may not be able to complete the necessary repeated measurements needed to perform statistical analysis and adequately assess sample heterogeneity. Solid sampling quantification is challenging when matrix-matched standards are not readily available and normally requiring the analyst to produce a matrix-matched standard.

It is common for liquid sampling volume requirements to reach up to a milliliter or more for repeated measurements of the sample for well-established techniques ICPMS and ICP-OES ${ }^{48,49}$. The measurement of very low concentrations of trace metals contained in very small volumes presents a considerable challenge to the analytical chemist.

Quantitative analysis of liquid samples by LIBS has posed to be rather challenging because, in part, the following inherent drawbacks. In liquids, the plasma radiation is weak because of the quenching by the water and weakening and broadening of the spectral lines as a result of collisions and the Stark effect ${ }^{50}$. Splashing from direct analysis of liquid surfaces may contribute to the extinction of plasma emissions, thereby resulting in shorter plasma lifetimes. The produced shockwaves increase the shot-to-shot variation, while splashing and the formation of bubbles created inside the liquid may produce quenching and change the characteristics of the plasma, thereby suppressing the LIBS signal ${ }^{51}$. Trying to reconcile for these disadvantages, different methods have been 
proposed including initiating the plasma within the liquid bulk of the sample to reduce splashing, however the plasma lifetime was extremely short under these conditions, approximately $1 \mu \mathrm{s}$ or less ${ }^{52}$. Other approaches for minimizing the drawbacks include the use of laminar flows ${ }^{52}$, jets ${ }^{53,54}$, freezing the samples in ice ${ }^{55}$, LIBS within cavitation bubbles $^{56}$, double-pulse plasma generation ${ }^{50,57}$, and converting liquid solutions into a solid matrix ${ }^{58}$. Previous research by Neimax ${ }^{59}, \operatorname{Hanh}^{60}$ and Jantzen ${ }^{61}$ have demonstrated that LIBS is capable of microdrop analysis.

The focus of the current research is to incorporate both aerosol analysis and for the first time, the use of microdrop printing in ambient conditions, to generate small droplets that can be entrained in a double-pulse LIBS plasma.

Continuous mode inkjet printing will be used to deliver microdrops containing $\sim 90$ picoliter volumes for LIBS analysis. Inkjet printing is a contactless method for depositing precise volumes of solutions and able to deliver drops ranging from a few microns in diameter to tenths of a millimeter ${ }^{62}$, depending on the nozzle orifice. Section 1.8 will present further details regarding microdrop printing.

The motivation of this research is to use LIBS to accurately and precisely analyze small volumes of solution, $\leq 300 \mu \mathrm{L}$ for aerosol analysis and $90 \mathrm{pL}$ for singlemicrodrops. These two methods eliminate the splashing and the sample preparation steps that were needed in the above discussed LIBS solution methods, as well as dramatically reduce the total volume needed for analysis.

It has been noted that conventional single-pulse LIBS is less sensitive than the competing atomic spectroscopic technique of ICP-OES that utilizes a sustained plasma as the excitation source but also requires much more analytical volume. In an effort to 
increase the LIBS sensitivity, double-pulse LIBS excitation will be explored. Different laser pulse configurations have contributed to improving the limits of detection (LOD), by increasing the LIBS signal ${ }^{63,64}$. These techniques use a combination of two lasers or two pulses from one laser. Both are spatially overlapped and the two laser pulses are focused and separated some nanoseconds to tens of microseconds and will be discussed in sections that follow. 


\subsection{Physics of Laser Ablation}

In principle, there are no sample size requirements and any type of solid sample can be ablated. Laser ablation requires almost no sample preparation thereby eliminating contamination issues that arise with chemical dissolution, required by some methods. Solution analysis requires approximately a few milligrams of sample that is needed for the digestion and a total volume of approximately milliliters of sample. Laser ablation removes nanograms to microgram quantities of mass, making the sampling technique virtually non-destructive. The laser ablation process for LIBS and LA-ICP-MS are identical, however optimized parameters for each system will vary as will the equipment required and detection capabilities. Laser ablation is becoming the leading technology for the chemical analysis of solid samples ${ }^{65}$.

Laser ablation has been defined by the processes of material melting, fusion, sublimation, erosion and explosion. Laser ablation is an involved process, the lasermaterial interaction causes mass to leave the surface in the form of ions, electrons, atoms, molecules, clusters and particles, with each of these processes separated in time and space ${ }^{66}$.

The three main processes for laser ablation are: 1) bond breaking and plasma ignition, 2) plasma expansion and cooling and 3) particle ejection and condensation ${ }^{67}$. The time period for these processes varies by orders of magnitude with electron absorption of the laser energy occurring at $10^{-15}$ seconds, to particle condensation occurring $10^{-3}$ seconds after completion of laser pulse. Figure 4 demonstrates these processes and corresponding mechanisms. The laser irradiance, pulse duration, thermal 
and optical properties of the sample material are critical properties and influence these processes.

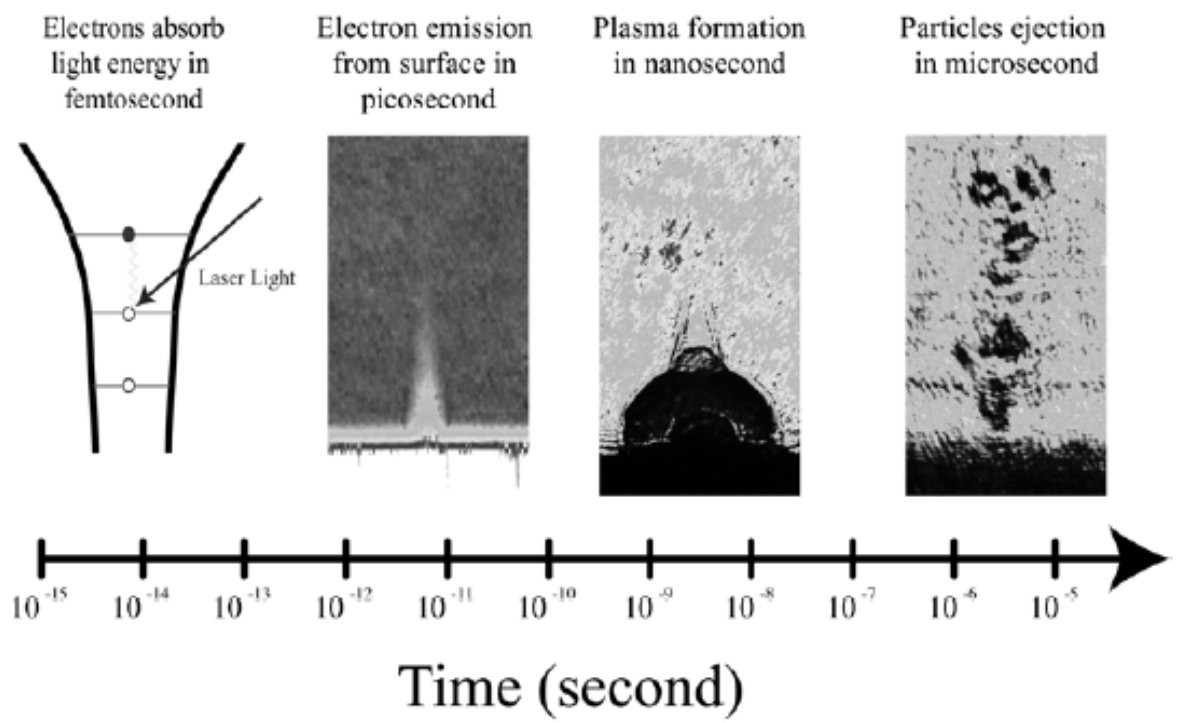

Figure 4. The time scale of events from laser pulse to particle ejection ${ }^{68}$

The dominant mechanism is thermal vaporization for a nanosecond laser pulse with irradiances less than $10^{8} \mathrm{~W} / \mathrm{cm}^{2}$. The temperature increases causing a transition from solid to liquid, liquid to vapor and vapor to plasma. The electron emission starts in the picosecond time frame, before the completion of the nanosecond laser pulse. Inverse Bremsstrahlung is dominant as the electrons emitted will gain energy from the incoming laser pulse through the collisions with the ionized vapor mass leaving the sample surface $^{69}$. When the kinetic energy of the electrons in the plume is higher than the ionization potential of the vaporized atoms, the plume may absorb the tailing end of the laser energy and partially shield the sample surface from further laser ablation ${ }^{70}$.

Picosecond laser pulse widths exhibit thermal and non-thermal mechanisms, such as Coulomb explosion, with irradiances between $10^{10}-10^{13} \mathrm{~W} / \mathrm{cm}^{2}$. Femtosecond pulses 
with irradiances greater than $10^{13} \mathrm{~W} / \mathrm{cm}^{2}$, Coulomb explosion is the dominant process ${ }^{67}$ in plasma formation.

Plasma shielding influences the amount of mass that is ablated into the LIBS plasma. In nanoseconds, the plasma will develop significantly. With a laser pulse width of less than 1 nanosecond, the absorption of laser energy into the developed plasma may be negligible. The picosecond width may partially be absorbed by an air plasma formed during the early stage electronic plasma ${ }^{71}$. The femtosecond pulse widths are neglected as a result of the pulse being too short to be absorbed by the plasma.

The electron density, electron temperature and the plasma expansion speed are highly dependent on the laser properties. The initial mass, energy and gas environment that the plasma is developed in will influence the expansion. These high laser energies cause the sample to transition rapidly from super-heated liquid to an explosion of vapor and liquid droplets.

When the absorbed laser energy is sufficient to fulfill latent heat of melting and vaporization, there is transfer of mass from solid into the plasma ${ }^{68}$. When the sample surface approaches a temperature several times the vaporization temperature, the laser energy can then significantly transfer more mass into the vapor phase. From this point the particles may then be swept into the ICP-MS. For LIBS analysis, an adiabatic plasma exists for approximately 1 microsecond where inverse Bremsstrahlung is the dominant factor, after this occurrence, the spectral line radiation is apparent for LIBS. 


\subsection{Introduction to Laser Induced Breakdown Spectroscopy}

Laser induced breakdown spectroscopy is an emerging method of atomic emission spectroscopy. A generalized LIBS setup requires: a pulsed laser, mirror, focusing and collection optics for the emitted radiation, and an optical fiber coupled to a spectrometer, see Figure 5.

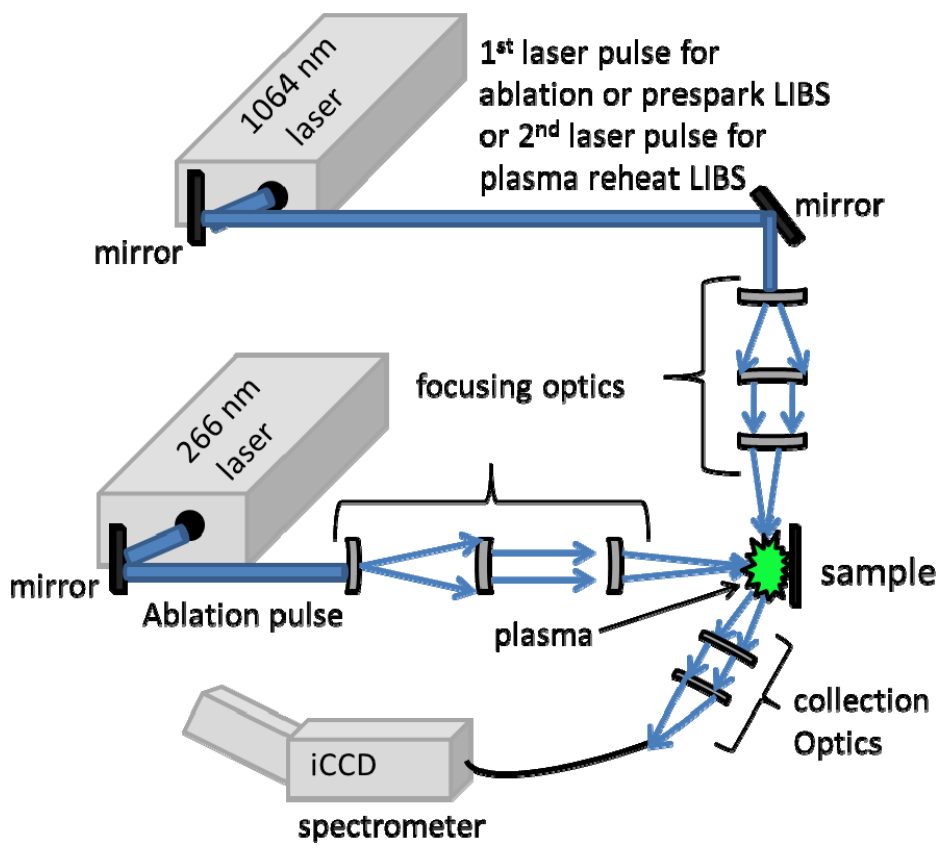

Figure 5. Single-pulse and double-pulse LIBS schematic

LIBS utilizes a high-powered laser pulse focused by a lens to a sub-millimeter spot on or in the solid, liquid or gaseous sample. A laser pulse of sufficient energy comes into contact with the sample surface that ablates a microgram or less quantity of material. The atomic and molecular structure of the sample is broken down inducing vaporization. The ablated material forms a plasma, in the form of molecules, neutral atoms, excited atoms, ions and electrons, which expands at a velocity much faster than the speed of 
sound, producing a shock wave in the surrounding atmosphere. Throughout this process there is a background continuum that decays more quickly with time than the spectral lines. The continuum is primarily the result of bremsstrahlung process in which photons emitted by the electrons is the dominating process ${ }^{72}$. During the following microseconds the plasma will decay through radiative quenching and electron-ion recombination processes ${ }^{72}$. Figure 6 demonstrates the timeline from laser pulse emission of spectral lines. It is usually at the microsecond time frame that the emission light is collected, transferred and detected. Each element has an exclusive emission spectrum; the wavelength corresponding to the emission line.

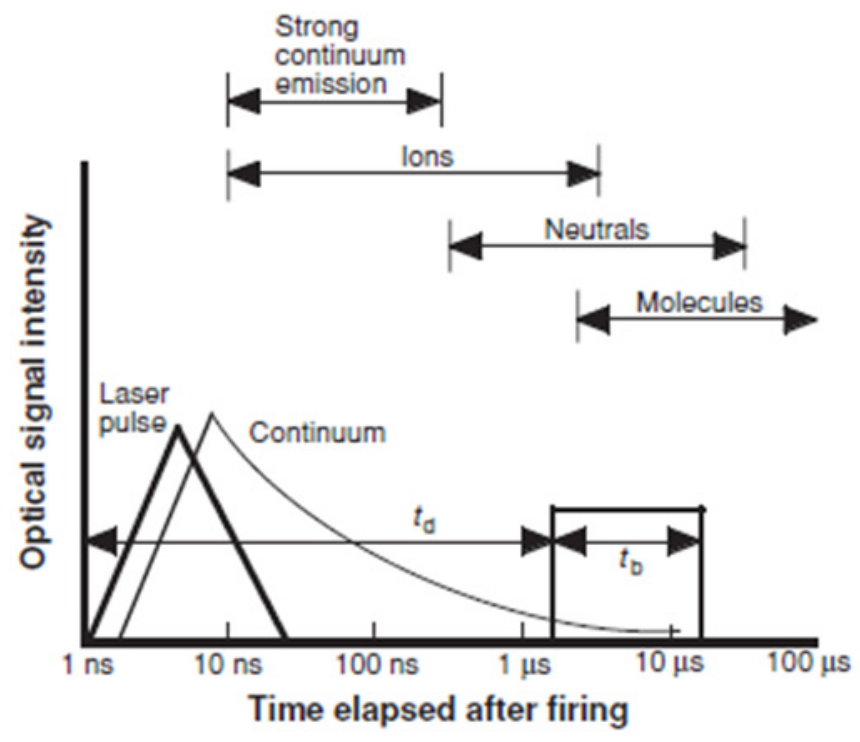

Figure 6. The timescale from laser pulse to spectral emission. Continuum decay time delay, $t_{d}$, represents the time before detector opening and $t_{b}$ represents the integration time $e^{72}$ LIBS typically removes nanogram to microgram quantities of mass ${ }^{31}$, classifying it as a surface technique. However, multiple pulses at the same location can be used to ablate the surface, thereby removing surface contamination and exposing underlying layers. The ablated mass is characteristic of the original sample, allowing the analyst to 
"fingerprint" the species. Each firing of the laser will produce a LIBS spectrum. The analytical signal is calculated by the integration of the emission line intensity or peak area. Typically, LIBS measurements are averaged or accumulated to increase precision and accuracy, and to average out signal variance.

\subsubsection{Development of LIBS}

Research began by spectroscopists with the invention of the laser in 1960 by Maiman ${ }^{73}$. The first LIBS plasma was reported by Brech and $\mathrm{Cross}^{74}$, where a laser was used to produce a plasma in 1962. Debras-Guedon and Liodec reported the first analytical use of LIBS on surfaces in $1963^{75}$. Shortly, proceeding the analytical accomplishment of LIBS on surfaces, the first instrument was produced based on the LIBS technique ${ }^{76}$. An auxiliary spark discharge was used to produce the light for spectral analysis. However, as a result of the poor accuracy and precision produced, this technique did not become very popular. During these early years the laser was predominantly used as an ablation source into a conventional plasma source. The detector used in the 1960 s to produce temporally resolved spectra were commonly the streak camera and rotating mirrors, until 1971 when Schroeder et al. developed a system to electronically gate and average the plasma signals ${ }^{77}$. However, it was not until the 1980 's that a renewed interest was developed in spectrochemical analysis by LIBS. This renewed interest of LIBS was contributed to the advances in instrumentation as the laser and other components of the LIBS system became smaller, the advantages became more apparent.

Laser induced breakdown spectroscopy has been referred to as laser induced breakdown plasma spectroscopy (LIPS) or laser spark spectroscopy (LSS). Over the last two decades LIBS has developed rapidly. Pioneers in LIBS research were the scientists at 
Los Alamos National Laboratory. They demonstrated in the early 1980s that LIBS was able to detect hazardous gases and vapors in $\operatorname{air}^{78}$ and small amounts of beryllium in air and on filters ${ }^{79,80}$. Proceeding from the research of the Los Alamos National Laboratory, many more LIBS applications were reported; liquids were analyzed at surface or in bulk $^{81,82}$, salt concentration was measured in sea droplets ${ }^{83}$ and carbon content in steel ${ }^{84}$ to list a few. With the new developments in lasers and detection devices, new applications have focused on practical problems, such as real-time monitoring of toxic metal emissions ${ }^{85}$, quality assessment of steal $^{86}$ and for planetary exploration the LIBS rover was developed by NASA ${ }^{87}$. In efforts to make LIBS more quantitative, many scientists have explored the fundamentals of LIBS, such as plasma fundamentals and laser ablation. These topics will be covered in the upcoming sections.

There has been considerable growth within the LIBS community since the development in the 1960's as LIBS has advanced in laboratories throughout the world. For instance, less than 50 papers were published per year from 1965 through 1990. However, there was a dramatic increase in LIBS publications from 2000 to 2005 with over 200 papers being published ${ }^{81}$. Currently, LIBS papers are appearing in prominent journals every month.

Commercial LIBS instruments are being manufactured by companies such as, Applied Spectra, Foster and Freeman, Applied Photonics and Photon Machines. These commercial LIBS systems have been purchased by analytical and forensic laboratories. The LIBS community has grown and developed international and national scientific conferences dedicated solely for the purpose of being able to exchange information and observe the contributions of other LIBS scientific research. 


\subsubsection{LIBS Plasma Fundamentals}

A plasma, the fourth state of matter, is composed of atoms, ions and free electrons and is overall electrically neutral. One of the main parameters for characterizing a plasma is the degree of ionization. The LIBS plasmas are typically weakly ionized as the ratio of electrons to other species is less than $10 \%{ }^{81}$. Hughes ${ }^{88}$ described two steps leading to breakdown threshold of the matrix as a result of optical excitation. First, a few free electrons serve as receptors to the laser energy, producing collisions with photons and neutrals. The second step is the background continuum. With a laser irradiance beyond $10^{9} \mathrm{~W} / \mathrm{cm}^{2}$ with a nanosecond or shorter laser pulse, an explosion occurs ${ }^{68}$. The sample surface rapidly heats, melts and vaporizes material as a result of the laser-material interaction with the leading edge of the laser pulse. As the energies of the electrons grow, ionization is produced through collisions, other electrons and energy absorption. The Bremsstrahlung process is a result of photons being emitted by electrons that are accelerated or decelerated in collisions. Recombination happens when a free electron is captured into an atomic or ionic energy level and the excess kinetic energy is released in the form of a photon. The continuum is a result of Bremsstrahlung and recombination events.

The laser induced plasma will continue to expand after the end of the laser pulse. The expansion of the plasma is dependent upon the amount of mass ablated, the energy and spot size of the laser and the surrounding environment.

The plasma expands outward from the focal volume with an initial rate of expansion on the order of $10^{5} \mathrm{~m} / \mathrm{s}$, the ablated mass compresses and a shock wave is produced that is audible to the analyst ${ }^{72}$. The early plasma expansion from a nanosecond 
laser on a float glass sample can be seen in Figure 7. This is a shadowgraph taken within the laboratory demonstrating the growth of the shock wave from $880 \mathrm{~ns}$ to $1.15 \mu \mathrm{s}$.

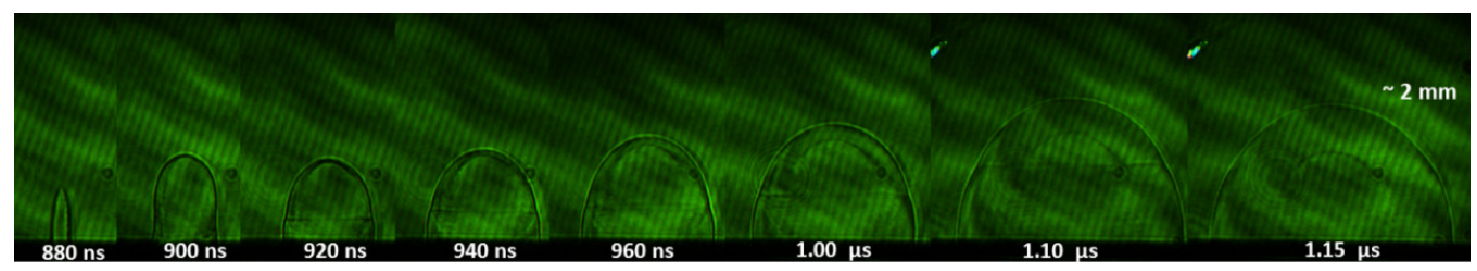

Figure 7. Shadowgraph demonstrating shockwave growth from 880 ns to $1.15 \mu \mathrm{s}$ When the pressure of the plasma plume equals the ambient pressure, the plasma expansion will stop.

The plasma evolves through many transient states from initiation to extinction. LIBS requires time resolution to avoid collection of the continuum and for the collection of light during the period that the analyte signal of interest is dominant. As the laser induced plasma cools, photons characteristic of the elemental composition of the sample surface are emitted. Neutrals are formed by the recombination of ions and electrons and other species recombine to form molecules as demonstrated in Figure 6.

Plasmas are diagnosed by their properties, such as plasma temperature and electron density, as well as their spectral lines. The spectral line intensities are dependent on the environment of the emitting atom. Lines are characterized by their wavelength, intensity and shape that are directly related to the density of the plasma and the electronic temperature. There are different broadening mechanisms for the spectral lines.

Natural broadening is a result of the excited states lifetime, which results in a Lorentzian profile. Doppler broadening arises as a result of the random thermal motions of the emitting atoms and results in a Gaussian line shape. However, Doppler broadening is negligible in comparison to the broadening being caused by charged particles. Stark 
broadening is dominant in LIBS plasmas ${ }^{89}$ because of the high electron densities, i.e., $\sim 10^{15}-10^{18} \mathrm{~cm}^{-3}$. Stark broadening is the interaction between the atom and the electric field, as the atom interacts with fast moving electrons and slow moving ions producing a Lorentz shape. This results in the line profiles being dominated by Stark broadening for the considerable time period ${ }^{70}$.

The intensity of spectral lines is dependent of the transition probability and on the excitation conditions. When the electron impact excitation of atoms is high, as in a LIBS plasma, the population of the excited states is in agreement with the electron temperature. Electron temperature is based on the assumption that local equilibrium exists. The LIBS plasmas are described by local thermodynamic equilibrium (LTE). For LTE to exist to collisional processes must be more dominant over the radiative processes. LTE will hold true for high electron density plasmas ${ }^{70}$. When plasmas are in the state of LTE, a temperature can be found using Boltzmann and Saha equation's relationship between excited and ionic states population density ${ }^{70}$. LTE can be assumed if

$$
\mathrm{N}_{\mathrm{e}}>>1.6 \times 10^{12} \mathrm{~T}^{1 / 2}\left(\mathrm{E}_{2}-\mathrm{E}_{1}\right) \mathrm{cm}^{-3}
$$

where $\mathrm{N}_{\mathrm{e}}$ is the electron density, $\mathrm{T}$ is electron temperature in $\mathrm{K}$, and $\mathrm{E}_{2}-\mathrm{E}_{1}$ is the energy difference in electron volts.

Plasma temperature has a high electron density at atmospheric pressure which allows the assumption of LTE and then the use of the Boltzmann and Saha equations ${ }^{90}$. If LTE is assumed then the electron temperature, $T_{e}$, can be assumed to be equal to the excitation temperature, $\mathrm{T}_{\text {exc }}{ }^{91}$. The Boltzmann equation provides relationships between transition line intensities from the same ionization stage and the Saha equation provides relationships between transition line intensities from different ionization stages. Both the 
Boltzmann plot and Saha-Boltzmann plot have been used by other researchers for the determination of excitation temperature ${ }^{92-94}$. The Boltzmann equation is used to determine the plasma temperature:

$$
\frac{N_{n}}{N}=\frac{g_{n} e^{\frac{-E_{n}}{k T}}}{\sum_{n} g_{n} e^{\frac{-E_{n}}{k T}}} \equiv \frac{g_{n} e^{\frac{-E_{n}}{k T}}}{Z(T)}
$$

where $N$ is the total number of atoms or ions per $\mathrm{m}^{3}, N_{n}, E_{n}$, and $g_{n}$ are the population density, energy, and statistical weight of the quantum state $n$.

The Saha equation is written as

$$
\frac{N_{e} N^{z}}{N^{z-1}}=\frac{2 Z^{z}(T)}{Z^{z-1}(T)}\left(\frac{m k T}{2 \pi \mathrm{h}^{2}}\right)^{\frac{3}{2}} e^{-\frac{E_{\infty}^{z-1}-\Delta E_{\infty}^{z-1}}{k T}}
$$

The ionization of the species is represented by the superscript, $N_{e}, E_{\infty}^{z-1}$, and $\Delta E_{\infty}^{z-1}$ is electron number density, the ionization energy of the species $z-1$ for isolated systems, and the correction to the ionization potential for interactions in the plasma ${ }^{90}$.

The Saha-Boltzmann expression is similar to the classical Boltzmann expression. Using this method the temperature is determined from the slope of the linear fitting of the plot, which is obtained using several spectral lines from different ionizations, in the same way that the Boltzmann plot provides temperature from only one ionization stage. The Saha-Boltzmann method increases the accuracy of the temperature calculation because it includes the emissivities of lines from different ionic species of the same element ${ }^{95}$. The Saha-Boltzmann equation is defined as

$$
\ln \left(\frac{\varepsilon_{z} \lambda}{A g_{j}}\right)=-\frac{1}{k T} E_{j}^{z} Z+\ln \left(\frac{h c N^{0}}{Q^{0}(T)}\right)
$$


where $z$ indicates the ionic species of the line emissivity, $\varepsilon . A$ is the transition probability $\left(\mathrm{s}^{-1}\right), \lambda$ the transition wavelength $(\mathrm{m}), \mathrm{g}_{\mathrm{j}}$ and $E_{j}^{z}$ are the static weight (adimensional) and the energy (J) of the upper level of the transition, $k$ the Boltzmann constant, $\left(\mathrm{J} \mathrm{K}^{-1}\right) T$ the plasma temperature (K), $h$ is Planck's constant ( $\mathrm{J} \mathrm{s}), c$ the speed of light $\left(\mathrm{m} \mathrm{s}^{-1}\right), N^{0}$ is the number density of the neutral atoms $\left(\mathrm{cm}^{-3}\right)$ and $Q^{0}(T)$ the partition function for neutral atoms (adimensional). The relationship for equation four is linear. The Saha-Boltzmann equation allows for a linear plot. A line fit to this equation has the slope of $-1 / k T$.

The Stark broadening of lines is a prevailing procedure for determining the electron density, $N_{e}$. Stark effects are caused by the electric fields produced by nearby ions and electrons; this is the prevalent cause of pressure broadening in plasmas ${ }^{90}$. Typical plasma densities in the LIBS community range from $10^{16}-10^{18} \mathrm{~cm}^{-3}$, and are within the range for Stark broadening to be a dominant mechanism for emission line broadening. The determination of Stark broadening does not require calculating the absolute intensities of the spectral lines, the line shapes and FWHM are sufficient. The spectrum produced by the laser-induced plasma is then fitted to the Lorentzian profile. The Lorentzian function is expressed as

$$
y=y_{0}+\frac{2 A}{\pi} \frac{w}{4\left(x-x_{c}\right)^{2}+w^{2}}
$$

where $w$ is the full width half $\max (\mathrm{FWHM}), x_{c}$ is the center wavelength, $y_{0}$ is the background emission and $A$ is the integrated area of the emission line. When the spectral line is fitted to the Lorentzian profile the FWHM is calculated. The FWHM of Stark broadened lines is related to the electron density and can be calculated by the following expression $^{14}$ 


$$
\Delta \lambda_{\frac{1}{2}}=2 W\left(\frac{N_{e}}{10^{16}}\right)+3.5 A\left(\frac{N_{e}}{10^{16}}\right)^{\frac{1}{4}}\left[1-\frac{3}{4} N_{D}^{-\frac{1}{3}}\right] W\left(\frac{N_{e}}{10^{16}}\right)
$$

where $\Delta \lambda_{\frac{1}{2}}$ (angstroms) is the FWHM, $N_{e}$ plasma electron density $\left(\mathrm{cm}^{-3}\right), A$ is the ion impact parameter (angstroms), $W$ is the electron impact parameter (angstroms), and $N_{D}$ is the number of particles in a Debye sphere and is estimated from the following equation

$$
N_{D}=1.72 \times 10^{9} \frac{T^{\frac{3}{2}}}{\sqrt{N_{e}}}
$$

$\mathrm{T}$ denotes the temperature in $\mathrm{eV}$. The first term on the right side comes from the electron interaction, while the remaining part of the equation is dependent on the ion interaction. The impact parameters, which correspond to the collisions between two charged particles, have been computed for different line temperatures and can be referenced ${ }^{90,96}$. It has been demonstrated ${ }^{91,96}$ that the LIBS ion contribution is negligible to the overall broadening and therefore the form of the equation becomes

$$
\Delta \lambda_{\frac{1}{2}}=2 W\left(\frac{N_{e}}{10^{16}}\right)
$$

Determination of the electron density by Stark broadening is independent of the assumptions regarding LTE conditions.

Self-absorption will broaden a peak and if severe, the peak will appear flattened. Self-absorption is encountered in high plasma densities when the plasma absorbs its own emission. Self-absorption is caused from resonance lines, when the transition is from the upper state being the lowest excited level to the ground state. Self-reversal occurs as the light passes through the lower temperature regions of the plasma. The spectral lines will potentially look like two lines as a dip forms in the middle. 
The LIBS measurements usually occur approximately a microsecond after the plasma formation. It is mostly in this time frame that one can assume LTE as the time delay will greatly affect the electron density and the excitation temperature. Electron densities are greater at atmospheric conditions because of the free plasma expansion in $\operatorname{air}^{70}$. Typically, when electron densities range from $10^{15}<\mathrm{N}_{\mathrm{e}},<10^{18} \mathrm{~cm}^{-3}$ and excitation temperatures between $0.5<\mathrm{T}_{\mathrm{e}}<2 \mathrm{eV}$, the LTE conditions will be satisfied and both Saha and Boltzmann equations will hold true ${ }^{70}$.

The LIBS spectra are recorded temporally to provide the highest signal-to-noise ratio corresponding to the best precision. Data collections from LIBS experiments were recorded after the laser pulse at a delay of approximately hundreds of nanoseconds to microseconds. Understanding the LIBS plasma expansion during this time period will aid the analyst in producing the optimized parameters for their LIBS measurements.

\subsubsection{Double-Pulse LIBS}

In addition, to single-pulse LIBS where only one laser is used for ablation and excitation, double-pulse combines two laser pulses. Different laser pulse configurations have contributed to better sensitivity, while gaining signal for LIBS analyses ${ }^{97-99}$. These pulses can be the combination of one laser or from two different lasers with an interpulse delay ranging from nanoseconds ${ }^{100}$ to microseconds ${ }^{97}$. Several double-pulse geometric configuration approaches have been used consisting of either one laser ${ }^{101}$, or two different lasers ${ }^{102}$ to encompass a double-pulse LIBS setup. Geometrical configurations include either collinear, in which the first and subsequent laser pulses are focused to the same location on the sample, or orthogonal. The orthogonal configuration includes one 
beam perpendicular to the surface while the other pulse is parallel to the surface.
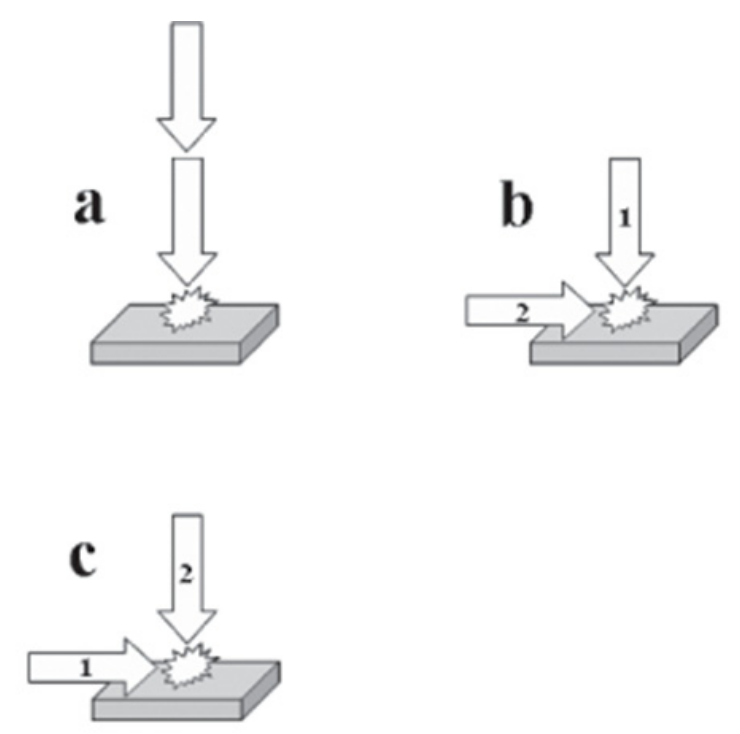

Figure 8. Schematic of the different double-pulse LIBS configurations: a) collinear, b) reheating orthogonal and c) prespark orthogonal $^{103}$

Within the orthogonal setup there are two configurations: pre-spark and plasma reheat. Pre-spark will pulse the beam parallel to the surface, first creating a spark in air followed by the perpendicular ablation pulse. Plasma reheat will pulse the beam perpendicular for ablation followed by the parallel pulse reheating the plasma. Figure 8 , produced by Scaffidi et al. ${ }^{103}$, illustrates the different double-pulse LIBS configurations.

The enhancement observed in collinear double-pulse LIBS has been contributed to an increase in mass ablation, plasma volume and the reheating of the plasma by the second pulse ${ }^{99,103,104}$. Orthogonal reheat enhancements have been contributed to higher plasma temperatures and electron densities as a result of larger plasma size from the second reheat laser pulse ${ }^{105}$. The enhancements of prespark LIBS are theorized to breakdown the ambient atmosphere prior to the ablation. The breakdown of the ambient 
atmosphere results in a larger and faster plasma expansion, while also producing less plasma shielding of the ablation pulse ${ }^{97,105-107}$. A more depth study of double-pulse LIBS for the analysis of glass will be covered in the upcoming section.

\subsubsection{Optimization of LIBS}

The main parameters for optimized performance are laser wavelength, laser pulse energy, lens-to-sample-distance (LTSD), gate delay (time from laser pulse to spectral line detection) and the integration time (time of collection). With optimized parameters, the analyst may improve the LOD, precision and accuracy.

The analytical signal for LIBS is calculated by emission line integrations. If the integrations of the emission line intensities or peak areas vary between replicates during experimentation, the accuracy and precision of the measurement may deteriorate.

Optimization of the listed parameters will lessen the shot-to-shot variance, thereby increasing the accuracy and precision.

Laser irradiance is an important parameter to be optimized. It is responsible for the effects caused by laser interaction with the sample and plasma evolution ${ }^{108}$. The sampling process of LIBS is ablation, the interaction of the laser energy with the sample. The ablation is responsible for the amount of mass removed from the sample by the laser pulse. The mass is atomized and ionized by the laser induced plasma from the energy supplied by the laser pulse. The mass in the laser induced plasma is responsible for the LIBS signal observed. Ablating a consistent amount of mass removed will improve precision of the LIBS analytical signal. Laser irradiance will be further discussed in Chapter 2. 
The lens-to-sample distance is defined as the depth of focus of the laser. The laser beam is focused at a normal incidence to the sample through a plano-convex lens to a sub-millimeter spot. The sub-millimeter spot may then be focused behind the sample surface. Optimization of the LTSD may increase signal while also producing better precision. It is important to find the optimized position and keep this position constant throughout the experimental measurements for accuracy and precision. It has been documented that adjusting this parameter may aid in the intensity of atomic and ionic emission lines ${ }^{31}$.

The laser induced plasma is a transient process. Time delays are important to monitor the plasma evolution. Ionization is high during the early plasma formation. During the plasma evolution the temporal gating is controlled to avoid the continuum and to capture the spectral emission lines, see Figure $6^{72}$.

Time resolved spectral detection is an important factor in achieving optimization as it has been demonstrated to enhance sensitivity ${ }^{109}$. Since the plasma evolution from continuum radiation to characteristic radiation has a lifetime of approximately $10 \mu \mathrm{s}$, temporally gating the detector and controlling the gate width allows the LIBS operator to view the plasma at a distinct time for a finite integration. Gating the spectrometer is important not to damage the intensified CCD array and will aid in reducing inferences, such as overlapping emission wavelengths ${ }^{81}$. Atomic line emission is most prevalent after the plasma expansion at lower temperatures as a result of the radiative recombination of the charged particles in the plasma ${ }^{70}$. As a result of the different decay rates of the continuum and the atomic species, the analyst may select the proper detection window when the analyte species emission is the highest. 
The LIBS signals are optimized for both the gate delay and the integration time to produce the highest signal-to-noise ratio, corresponding to the best precision and accuracy.

\subsubsection{Advantages of LIBS}

Simplicity is an important advantage for LIBS, as is being able to analyze all types of materials. Gases, liquids and solids can be analyzed quickly and accurately, with experiments performed at atmospheric pressure. LIBS is a micro-sampling technique and is considered to be virtually non-destructive. The total mass removed per LIBS analysis is in the nanograms range ${ }^{31}$, allowing for evidence preservation in the forensic laboratory. Little-to-no sample preparation is required as a few laser pulses prior to analysis will remove all contaminants. LIBS is able to provide multi-element detection with no prechosen element menu.

An echelle spectrometer covers a very broad spectral range, i.e., 200-900 nm, providing multi-element analysis, with excellent resolution using LIBS. LIBS sampling allows for different analytes of interest to be monitored simultaneously. LIBS provides real-time elemental analyses, with the possibility of in-situ analysis and stand-off detection ${ }^{110}$. LIBS is often referred to as a semi-quantitative technique, however, recent research has demonstrated LIBS to be capable of quantitative analysis ${ }^{111-113}$.

\subsubsection{Disadvantages of LIBS}

Chemical matrix effects may interfere with the calibration and occur when the emission of one element affects the precision of another element. These matrix effects can be 
corrected if the concentration of the interfering element is known or by choosing another emission line. Special care must be given to non-homogeneous samples. LIBS is a micro technique and when analyzing liquids or gases it is often safe to assume the sample is heterogeneous. Solid analysis cannot assume homogeneity; special attention needs to be given to ensure one is collecting a representative elemental profile of the sample.

Representative sampling can be accomplished by sampling at many locations repeated times or to mill the samples and press the resulting powder. LIBS is criticized for not being as sensitive as the other well-established techniques. The sensitivity is largely dependent on the spectrometer used for analysis. However, research has shown LIBS to compete with LA-ICP-MS in the analysis of forensic glass ${ }^{3}$.

Another disadvantage often associated with LIBS is the shot-to-shot variability, that is often contributed to the laser-sample interaction ${ }^{72}$. The lack of matrix matched standards may pose difficult challenges during the solid sampling examination process. Matrix-matched standards are important for quantitative analysis. It is assumed that the laser-material interaction in matrix-matched samples will be similar, therefore removing similar amounts of mass ${ }^{114}$, thereby producing a similar analytical response and allowing for quantitative analysis. The laser-material interaction will be significantly different between different sample matrices, which require LIBS to use an external calibration.

Many steps are often taken in preparing homogeneous solid samples because they are often unavailable for purchase. For example, pelletizing is the most common way of solid sample preparation. The matrix is ground into a homogeneous powder, which is then pressed into a pellet ideally producing a flat, homogeneous surface. Milling of the sample has been documented to produce homogenous samples ${ }^{115}$. Shot-to-shot variability 
is also overcome by normalization, either to an internal standard or to another emission line within the spectrum and with parameter optimization.

Overall, LIBS is proving to be a competing technique for the elemental analysis of solids, liquids and gases. 


\subsection{Principles of Laser Ablation Inductively Coupled Mass Spectrometry}

Laser ablation inductively coupled mass spectrometry is one of the most successful solid sampling techniques. LA-ICP-MS provides a wide linear dynamic range for detection of major, minor and trace elements.

In 1980, Houk et al. ${ }^{116}$ introduced ICP-MS and the basic set-up has not changed since then. Improvements were achieved in producing lower backgrounds, better sensitivity, user-friendly interface, reduced size and reduced expense ${ }^{117,118}$. The ICP-MS includes five main parts: 1) Sample introduction, 2) Plasma in torch, 3) Sampler and skimmer cone interfacing between atmospheric pressure and vacuum, 4) Mass spectrometer and 5) Detector ${ }^{119}$.

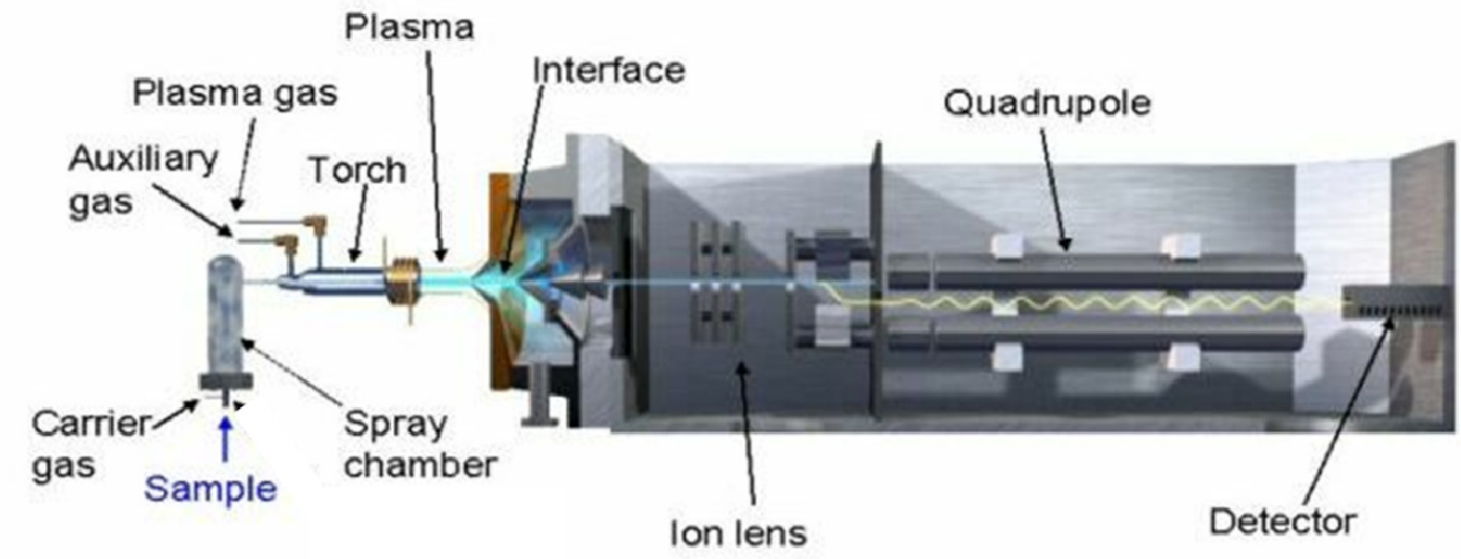

Figure 9. Schematic of ICP-MS instrument ${ }^{119}$

Precision and accuracy is dependent on sample introduction. Sample introduction was initially only for solutions in which a nebulizer produced an aerosol. The aerosol was then transported by a carrier gas into the ICP. The experimental procedure requires all samples to be in solution. In 1985, Gray ${ }^{39}$ developed laser ablation as a new sample 
introduction for ICP-MS. Laser ablation generates particles when the laser-material interaction occurs with the solid sample. A carrier gas then sweeps these particles in the ICP-MS. Today, laser ablation, has eliminated sample preparation time consuming dissolutions, while still providing the sensitivity of ICP-MS. The laser ablation process is reviewed in more detail in section 1.5.

The ionization source is an inductively couple plasma (ICP) torch. It was originally used as an excitation source for optical spectroscopy. The torch consists of three concentric tubes through which argon flows, varying from 5 to $20 \mathrm{~L} / \mathrm{min}^{120}$. A high radiofrequency is applied to the coil and the argon is ionized by a spark from the Tesla coil. Collisions of the electrons and the resulting ions sustain the plasma. The interaction between the magnetic field and the electrons and ions result in an annular path motion. The plasma is maintained and the torch is protected from melting by different gas flows. The tangential flow of the argon cools the inside walls of the center tubes and centers the plasma. The atoms are ionized by the electron impacts. The atoms will have a resonance time in the plasma for approximately 2 milliseconds at temperatures from $4000-8000$ $\mathrm{K}^{120}$. The ionization potential of the atoms will determine the degree of ionization. The ionization potential of argon is $15.6 \mathrm{eV}$. All elements with a significantly lower ionization potential will be $90 \%$ or more ionized ${ }^{118}$.

The first ion extraction occurs in the interface coupling between atmospheric pressure of the torch and vacuum pressure of the mass spectrometer. The connection between the torch and the mass spectrometer is a critical part of the instrument and consists of the sampling and the skimmer cone. The skimmer cone with a diameter of approximately $1 \mathrm{~mm}$ is water cooled because of the high temperatures of the plasma. 
Following this cone, the pressure is maintained at approximately 1 Torr by a rotary pump. The ions expand rapidly in this region and are cooled. A fraction of these ions then pass through the skimmer cone with a diameter of typically $0.8 \mathrm{~mm}$. Behind the skimmer cone the pressure is maintained at the same pressure of the mass spectrometer using turbomolecular pumps. The ions are separated by a negative potential. The lighter ions are deflected more than the heavy ions as a result of the electrostatic repulsion of the positive charged ions. The remaining ions are then guided in this region by a magnetic ion lens to the entrance of the mass analyzer where they are separated by their mass to charge ratio.

\subsubsection{Optimization of LA-ICP-MS}

The optimization procedure consisted of measuring and achieving certain target values for certain isotopes. The isotopes and their minimum corresponding values are listed first by isotope, followed in the parenthesis by the minimum target value listed in counts per second. The following isotopes were optimized: ${ }^{7} \mathrm{Li}(>1500 \mathrm{cps}),{ }^{49} \mathrm{Ti}(>1000 \mathrm{cps}),{ }^{57} \mathrm{Fe}$ (>8000 cps), ${ }^{59} \mathrm{Co}(>8000 \mathrm{cps}),{ }^{139} \mathrm{La}(>10000 \mathrm{cps}),{ }^{140} \mathrm{Ce}(>14000 \mathrm{cps}),{ }^{232} \mathrm{Th}(>3000$ cps), ${ }^{238} \mathrm{U}(>3000 \mathrm{cps}), \mathrm{ThO} / \mathrm{Th}$ fraction needed to be less than $1 \%$, doubly charged interferences needed to be less than $3 \%$, and $\mathrm{U} / \mathrm{Th}$ was sufficient between $0.7-1.3$. The instrument is determined to be functioning properly when the above criteria are met.

\subsubsection{Advantages of LA-ICP-MS}

The introduction of laser ablation to ICP-MS created extra advantages to already the existing advantages of ICP-MS as a solution analytical technique. First, the sample 
preparation is greatly reduced as dissolution methods may take several hours with potentially hazardous chemicals. There is less sample handling and reducing contamination. Contamination may be a result of sample containers, solvents and/or the atmosphere. Molecular ion inferences are reduced with no solvents being needed, as in solution analysis. Solid sampling by LA-ICP-MS provides spatially resolved information and depth resolution capability. Total mass ablated is usually nanograms to micrograms ${ }^{114}$, making the technique virtually non-destructive. Traditional solution methods require milligrams of sample.

LA-ICP-MS is able to offer rapid simultaneous, multi-element detection reaching part per trillion limits of detection with a wide linear dynamic range.

\subsubsection{Disadvantages of LA-ICP-MS}

LA-ICP-MS is a micro sampling technique and consumes much less mass quantities than that of ICP-MS. Sampling at many random locations has been shown to provide a representative elemental profile of solid samples ${ }^{41}$. Solution techniques are known for their tedious, time consuming wet chemistry methods. However, it is possible to produce homogeneous samples with matrix-matched standards.

Similar to LIBS, the lack of matrix matched standards produces difficult challenges during the solid sampling examination and quantitation process. Often numerous steps, such a pelletizing, are used to prepare homogenous solid samples. The elemental sensitivity will be significantly different between different sample matrices, which require LA-ICP-MS to use an external calibration. It is assumed that the laser- 
material interaction in matrix-matched samples will be similar, therefore removing similar amounts of mass ${ }^{114}$ and allowing for quantitative analysis.

Elemental fractionation is defined as the non-stoichiometric effects that occurs during laser ablation and is problematic without matrix matched standards. Fractionation prevents an accurate determination of the elemental concentration. Fractionation has been contributed to but not limited to: ablation, aerosol transport and excitation/vaporization. Mank et al. suggested that fractionation is a result of crater depth to crater diameter ratio which will affect particle ejection and particle melting ${ }^{121}$ and Horn et al. contributed it also to crater diameter ${ }^{122}$. Enhanced sensitivity was reported by Eggins et al. by using a helium/argon mixture as the carrier gas ${ }^{123}$. Laser ablation is responsible for the particle size and Houk et al. demonstrated that not all particles are vaporized within the ICP ${ }^{124,125}$. Russo et al. demonstrated that greater laser irradiances and shorter pulse widths decreases fractionation by producing smaller size particles which are transported more readily and efficiently ionized and atomized by the inductively coupled plasma ${ }^{114}$. 


\subsection{Microdrop Printing}

Microdrop printing is a contactless method for depositing precise sub-nanoliter volumes of solutions and is able to deliver drops ranging in diameter from a few microns to tenths of a millimeter, depending on nozzle orifice. Verkouteren et al. reported the standard uncertainties for microdrop dispensing to be approximately $1 \%$. Microdrop printing has the ability to deliver a wide range of solvent ${ }^{126}$ and aqueous ${ }^{127}$ based solutions, making the microdrop dispensing technique common in many analytical applications.

Microdrop printing from a nozzle was documented as early as 1833 by Savart ${ }^{128}$ and in 1878 Rayleigh $^{129}$ where they described the continuous mode inkjet printing technique. Here a pressure wave inside the capillary pushes the fluid from the nozzle orifice to produce uniform sized microdrops. As the drops fall into an electrostatic field, they acquire a charge and are focused to a location on the substrate. The variation in the charge applied will influence the microdrop trajectories.

Development of continuous mode inkjet printing continued to flourish. Dr. Sweets of Stanford University demonstrated a similar concept as above, the difference being that the fluid stream can be fragmented into microdrops of uniform size and spacing with an applied pressure wave $\mathrm{e}^{130}$. The microdrops that acquired a charge in the electric field were focused into a reservoir for recirculation and the uncharged drops would fall onto to substrate ${ }^{130}$. IBM licensed this technology in the 1970's to develop the technology for their computer printers.

A significant development by Hansell in 1950 patented the production of drops by an induced pressure wave ${ }^{131}$. A voltage pulse is applied to a piezoelectric material 
resulting in a volumetric change causing pressure in the fluid to produce a drop from the nozzle orifice. This documented the drop-on-demand mode, as the drop was only produced by an applied voltage. This technology flourished in the late 1970's as it was simpler than the continuous mode inkjet printing as a result of no fluid recirculation. The invention of drop-on-demand was also used for commercial printers and the ink microdrops were delivered by pressure wave of the piezoelectric ceramic.

Today drop-on-demand inkjet systems are more commonly used than continuous mode systems. However, both continuous and drop-on-demand modes are widespread. Continuous mode inkjet printing is mostly used industrially for product labeling of foods and medicines and drop-on-demand mode inkjet printing are most commonly used in the typical office printer.

Microdrop printing has many characteristics that make it appealing for many different analytical applications. It is a non-contact method that is not dependent on how wet the printed substrate becomes. The source of the fluid cannot be contaminated by the matrix during the inkjet dispensing. With the documented error rate of approximately $1 \%$ for drop production ${ }^{126}$, very precise volumes and mass quantities can be deposited.

Inkjet printing is currently being used for many applications. Sirringhaus et al. used inkjet printing on thin film transistors to produce electrodes on a glass substrate. They created channel lengths from sub micrometer to tens of nanometers ${ }^{132}$. Inkjet printing is also used for combinatorial studies because it is a direct writing process as it does not require the use of writing masks. Tekin et al. examined the emission properties using inkjet printing of different polymers with different side chains and film thicknesses ${ }^{133}$. Inkjet printing has been used to print organic, inorganic and hybrid 
inorganic/organic solar cells ${ }^{134,135}$. The deposition of nanoparticles using inkjet printing is becoming popular. Voit et al. deposited magnetic nanoparticles of iron oxide ${ }^{136}$. Crowley et al. printed nanoparticles to produce an ammonia sensor ${ }^{137}$. Microdrop printing has been used extensively for a long period of time in biological applications, as a result of vesicles being nanometer size. Vesicles are used in drug screening for living cells and play an important role in endocytosis and exocytosis processes ${ }^{138,139}$.

A demand in analytical chemistry exists to develop techniques for the analysis of ultra-small volumes. However, there is less research conducted in analytical chemistry using microdrops for chemical analysis. Microdrop printing allows for sub-nanoliter volume delivery. Currently, most research in this area has been conducted by Hahn and Niemax. These authors have demonstrated that microdrops deposited on Si-wafers can be used for analyte standards ${ }^{140}$. They conducted further studies with microdrops using analytical plasmas of ICP and LIBS. The microdrops were deposited into the plasma and linear calibration curves were constructed for each of both ICP and LIBS, demonstrating the potential for single drop analysis ${ }^{141}$. Pioneering research presented in Chapter 5 , demonstrates that LIBS is capable of microdrop quantification analysis using picoliter volumes $^{113}$.

\subsubsection{Piezoelectric Microdrop Printing}

The piezoelectric inkjet can be classified into four different categories: squeeze, bend, push and shear ${ }^{142}$. The bend mode forms a transducer by bonding the piezoceramic plates to the diaphragm used to form the microdrops. Piezoceramic rods expand to push against the fluid and eject microdrops in the push mode. In the shear mode the 
microdrops are formed and ejected by the deformation of the piezoplates pushing against the fluid. The squeeze mode, the most successful design, the glass nozzle is surrounded by a thin tube of piezoceramic. In the squeeze mode with the tubular piezo actuator surrounding the glass capillary, a voltage is applied to abruptly compress the enclosed fluid and produce a pressure wave that propagates through the glass capillary into the fluid. The pressure wave travels though the fluid and accelerates in the region of the nozzle, resulting in the ejection of a microdrop at the nozzle orifice that will fall freely in air.

Monodisperse microdrops are formed under optimized conditions. The following parameters are optimized though trial and error: drive pulse amplitude, drive pulse shape, internal pressure level, drop ejection rate and the fluid fill level. The drive amplitude will affect the size of the drop and the drop ejection velocity. The microdrop ejection speed will vary from approximately 1 to $10 \mathrm{~m} / \mathrm{s}^{143}$. Optimization of the pressure pulse rise and fall times will produce satellite free microdrops. Satellites or randomly sized drops will be formed if the amplitude of the pulse is too great.

The drop ejection process is determined by the drive waveform used for the piezoelectric actuator, see Figure 10. The optimum conditions are defined by achieving the highest drop velocity for the amplitude pulse. The first half of the waveform is controls the fluid expansion, the delay for pressure wave propagation and fluid compression. This creates the negative pressure initially and creates a pressure rise. The second half of the waveform is to cancel residual oscillations that remain after drop ejection $^{144}$. 


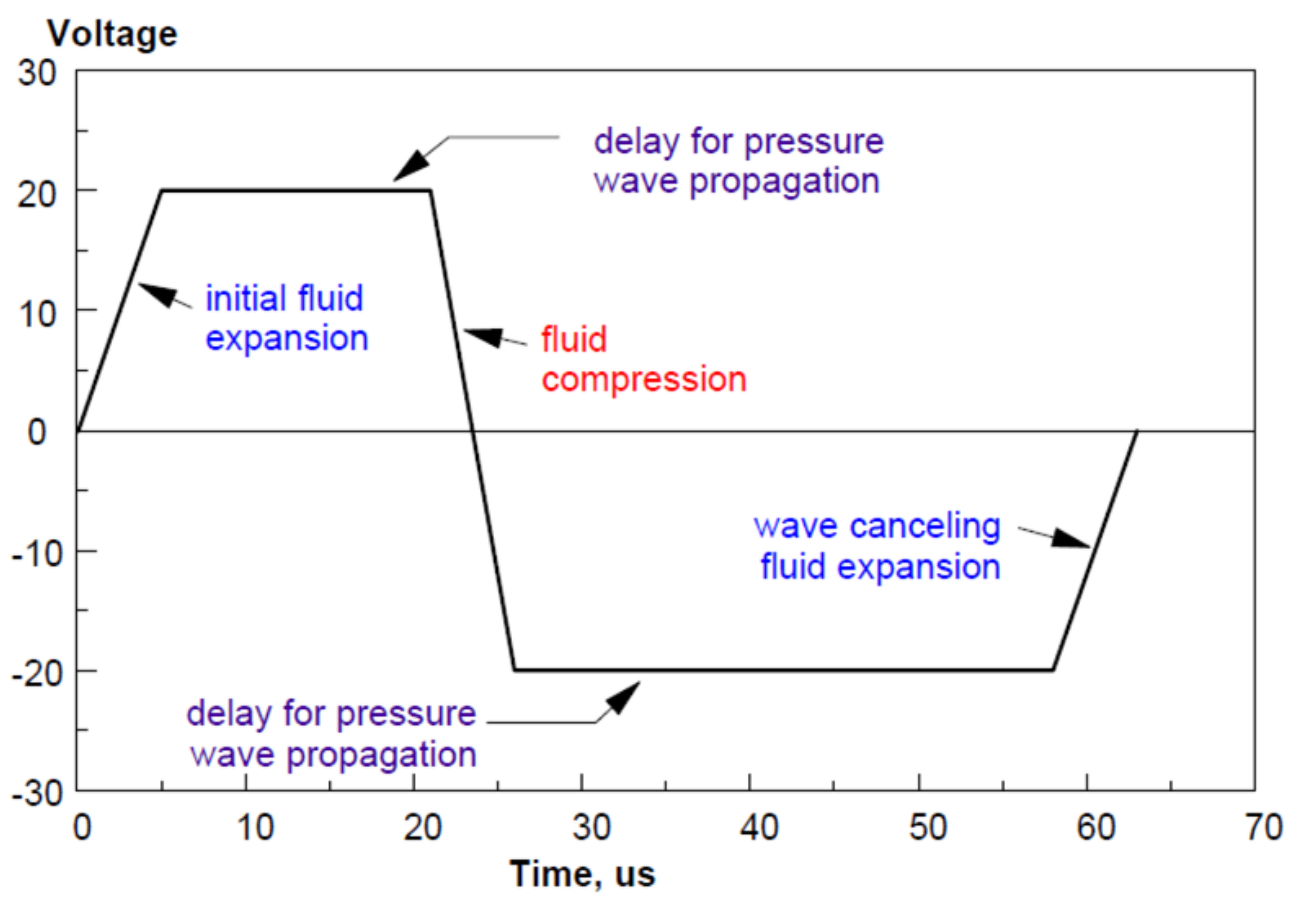

Figure 10. Piezoelectric waveform for inkjet printing ${ }^{144}$

Through trial and error the parameters were optimized. It was noted that shorter pulse widths will produce smaller drops, higher amplitudes are needed to eject microdrops with shorter pulse widths, the microdrops are primarily determined by the diameter of the nozzle orifice, changing the pulse width can alter the microdrop direction and a wet nozzle orifice may stop the production of drops.

\subsubsection{Terminal Velocity of Microdrops}

The atmosphere is made up of gas molecules, as an object moves through the atmosphere; these molecules are disturbed and will move around the object. It is easier to explain the interaction of two solid objects. When two solid objects interact, forces are transmitted at the point of contact. However, when a solid is immersed in a fluid media, such as air, the fluid is able to maintain contact at all points by flowing around the object. 
If the fluid is in motion, the direction of this motion can be defined. The lift is defined as the net force perpendicular to the flow direction and the drag is the net force with the direction of flow. These same forces are generated between the atmospheric gases and an object, such as a microdrop. The magnitudes of these forces are dependent of the object shape and speed and the mass and viscosity of the gas. The Reynolds number, $R_{e}$, is a measure of the viscosity and defined as

$$
R_{e}=\frac{v d}{v} \propto \frac{\text { dynamic pressure force }}{\text { viscous force }}
$$

where $v$ is the drop velocity relative to air, $\mathrm{d}$ is the drop diameter, $v$ is the kinematic viscosity (air is $0.151 \mathrm{~cm}^{2} / \mathrm{sec}$ at standard temperature and pressure) which equals $\eta / \rho$ where $\eta$ is the viscosity of air $\left(1.82 \times 10^{-6} \mathrm{gram} / \mathrm{cm}-\mathrm{sec}\right.$ at standard temperature and pressure $)$ and $\rho$ is the density of air $\left(0.001206 \mathrm{gram} / \mathrm{cm}^{3}\right)^{143}$.

In fluid mechanics the Reynolds number, represents the ratio of inertial forces to viscous forces in a fluid. The Reynolds number is dimensionless and high values indicate viscous forces are small, low values indicate that viscous forces dominate.

Many applications involve the drag coefficient of a sphere for spherical or nearspherical object, i.e., particles and droplets. The flow around the sphere is laminar and for Reynolds numbers of less than 0.5 , the force required though a viscous fluid at a specific velocity is defined by Stokes' Law ${ }^{145}$. George Gabriel Stokes discovered that when a spherical object falls through a fluid medium, the first layer of the fluid adheres to the spherical object and will travel along with at the identical velocity. It is a linear process as the next layer will have less velocity and so on, while the layer at furthest distance will remain at rest. The results are a dragging force upward, opposing the 
downward motion of the spherical object, called the viscous force ${ }^{145,146}$. The velocity of the falling object will increase as does the viscous force till the viscous force equals the body weight. This is known as terminal velocity. Stokes defined the viscous force by

$$
F_{d}=6 \pi \eta r v
$$

where $F_{\mathrm{d}}$ is the drag force (resistance) of the fluid on the spherical object, $\eta$ is the viscosity of the fluid, $r$ is the drop radius and $v$ is the drop velocity. To better understand this concept a free body diagram of a sphere is constructed. The sphere demonstrates the different forces, internal and external, that are encountered in the fluid (e.g., air), see Figure $11^{147}$.

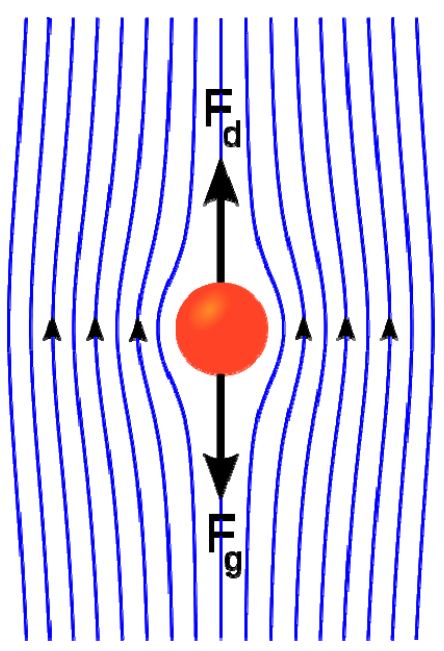

Figure 11. Stokes sphere forces ${ }^{147}$

The sphere seen in Figure 11 has three acting forces, two being upward forces and one being from the downward force. The two upward forces are a result of: 1) the displacement of the weight of the fluid, also referred to as the buoyancy effect ${ }^{146}, \mathrm{~F}_{\mathrm{b}}$, acting vertically upwards and 2) the viscous/drag force resisting the gravity acceleration, $F_{d}$. The only downward force is the weight of the spherical object falling. It is defined by the gravitation attraction $(\mathrm{F}=\mathrm{mg})$. The terminal velocity of a microdrop in air is 
calculated by setting both the upward drag forces equal to the gravitational force on the $\operatorname{drop}^{143}$. The sum of all forces equals

$$
F_{d}+F_{b}=m g
$$

where $m$ is the mass of the spherical object and $g$ is the gravitation force $\left(9.807 \mathrm{~m} / \mathrm{s}^{2}\right)$. Knowing that the volume of a sphere is given by

$$
v_{\text {sphere }}=\frac{4}{3} \pi \mathrm{r}^{3}
$$

and combining this with the $\rho$ being the density of the material and $\sigma$ being the viscous density of the medium, the following two equations are developed

$$
\begin{aligned}
& m g=\frac{4}{3} \pi r^{3} \rho g \\
& F_{b}=\frac{4}{3} \pi r^{3} \sigma g
\end{aligned}
$$

Therefore the combination of these equations produces

$$
F_{b, m g}=\frac{4}{3} \pi r^{3}(\rho-\sigma) g
$$

After rearranging the above terms, the terminal velocity, $v$, is solved for

$$
v=2 r^{2}(\rho-\sigma) g / 9 \eta
$$

The viscosity of the medium, in the case of microdrops is air. Stokes resistance is the energy that is required to orderly deform the air so that the microdrop can pass through.

Stokes drag force for microdrops is first order independent and is linear with velocity ${ }^{143}$. The Stokes drag force for microdrops is different from dynamic pressure sources associated with large objects, such as an aircraft. The drag at high Reynolds number $\left(\mathrm{R}_{\mathrm{e}}>10^{3}\right)$, is dependent on the square of the velocity. The terminal velocity of microdrops will be discussed further in section 5.5.2. 


\section{Wavelength Dependence of the Forensic Analysis of Glass by Laser Induced Breakdown Spectroscopy}

Most LIBS studies and commercial instrumentation utilize the IR $1064 \mathrm{~nm}$ laser, as a result of its higher power and lower cost of acquisition. However when searching through literature, it is the lower wavelengths that provide better analytical figures of merit for LA-ICP-MS studies ${ }^{148}$. The preferred laser wavelength for most LA-ICP-MS instrumentation is the fourth or fifth harmonic as it provides better laser-material interaction. It is for reason, I have investigated and compared the UV (266 nm) laser and the more widely used IR (1064 nm) wavelength for LIBS analysis of float glass ${ }^{31}$.

\subsection{Experimental Instrumentation}

The LIBS instrumentation presented in this work consisted of different single-pulse and double-pulse wavelength configurations. The LIBS system built in our laboratory consists of a Q-switched Nd:YAG NewWave Research Tempest laser (New Wave Research, Fremont, CA) operating at the fourth harmonic $(266 \mathrm{~nm})$ and a second Nd:YAG Big Sky laser (Big Sky, MT) operating at the fundamental wavelength of 1064 $\mathrm{nm}$, both having pulse widths of 3-5 ns full width half max. Flashlamps and Q-switches were both externally controlled by a Berkeley Nucleonics (San Rafael, CA) delay generator model 656. Beam expanders were used to enlarge the beams from $\sim 4 \mathrm{~mm}$ to nearly $12 \mathrm{~mm}$ using a Galilean telescope. The beams were then focused at a normal incidence to the sample through a plano-convex lens with a focal length of $150 \mathrm{~mm}$, see Figure 5, with a $90^{\circ}$ viewing angle to the laser. 
To ensure representative sampling and to account for any heterogeneity, each glass standard was analyzed 5 times at different locations. Both single-pulse and double-pulse spectra were collected as a result of 100 laser shots with the accumulation of the last 50 shots used to generate the spectra for analysis conducted at atmospheric pressure in air.

In order to gain the best reproducible optical emission spectra possible, both the $266 \mathrm{~nm}$ and the $1064 \mathrm{~nm}$ experiments were optimized for laser power, lens-to-sample surface-distance (LTSD), gate delay, and integration time.
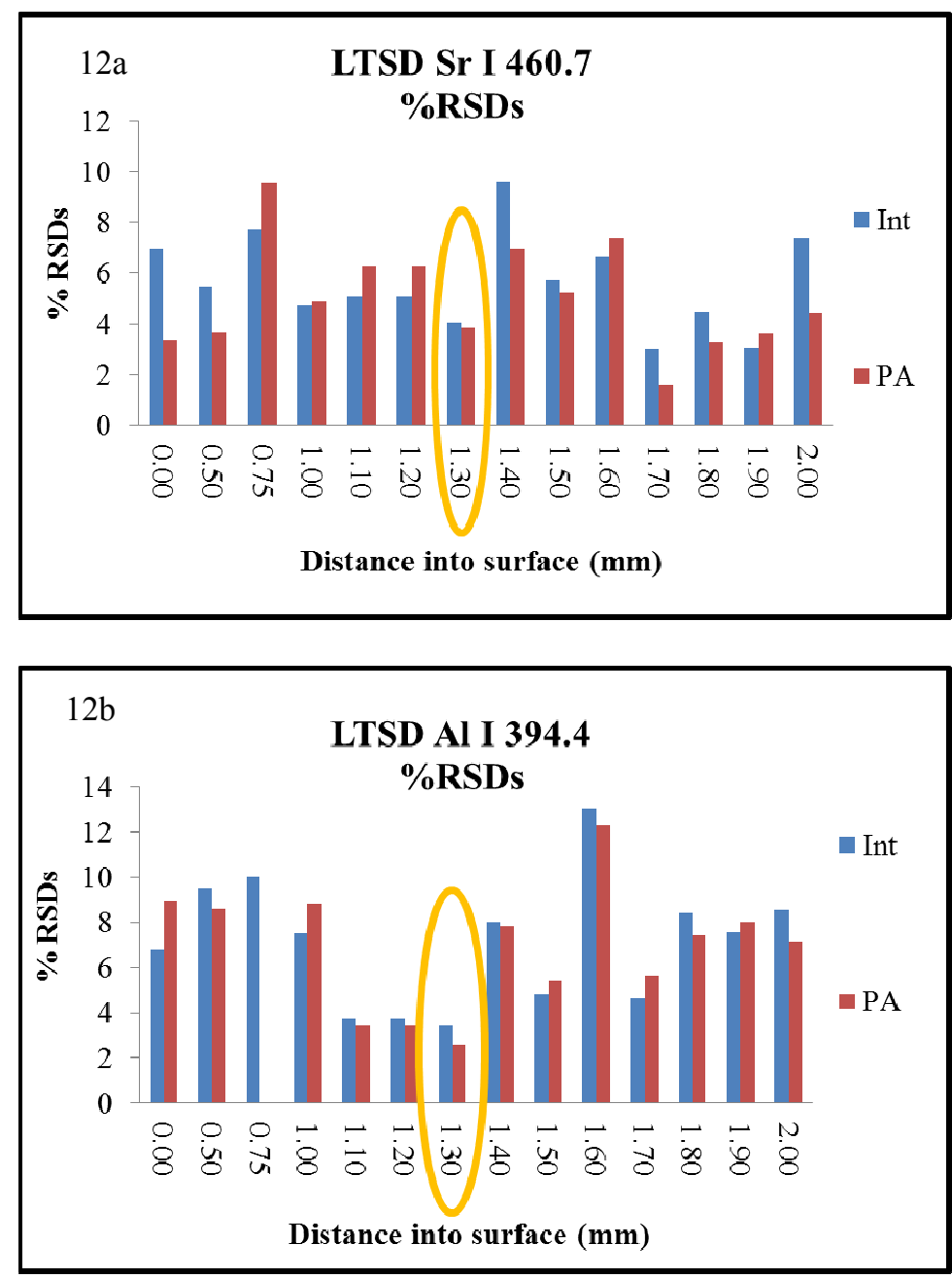

Figure 12. Lens-to-sample distance optimization for LIBS glass analysis for background subtracted intensity or Lorentzian fit peak areas for a) Sr I $460.7 \mathrm{~nm}$ or b) Al I $394.4 \mathrm{~nm}$ 
The laser energies for the single-pulse UV ablation was held at constant values throughout the experiments at $29 \mathrm{~mJ}$ for the $266 \mathrm{~nm}$ laser. The laser was focused 1.30 $\mathrm{mm}$ into the sample surface during the $266 \mathrm{~nm}$ experiment. Figure 12 demonstrates that the best precision was achieved for the $266 \mathrm{~nm}$ laser at a LTSD of $1.3 \mathrm{~mm}$ for both background subtracted intensity and Lorentzian fit peak areas.

The laser energy for the single-pulse IR 1064 ablation was held at constant value throughout the experiment of $47 \mathrm{~mJ}$. Figure 13 demonstrates the precision with the variance of laser energy. In forensic science, evidence preservation is important, and with an energy above $47 \mathrm{~mJ}$ the colorless glass fragment would be destroyed. Figure 17 shows the damage that is caused by IR $1064 \mathrm{~nm}$ laser interaction with colorless glass and the irregular crater morphology that is produced.

The IR $1064 \mathrm{~nm}$ laser was focused $0.50 \mathrm{~mm}$ into the surface for the $1064 \mathrm{~nm}$ experiment. The optimized energy and LTSD allowed for the least destruction of the sample while still providing analytical signal.

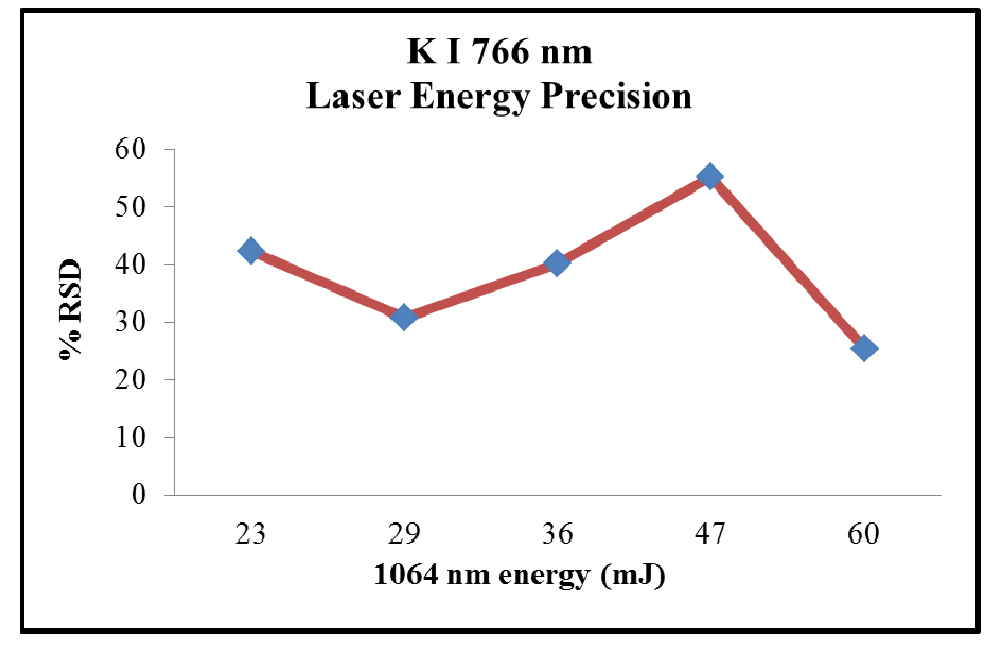

Figure 13. Precision variation with laser energy on glass using $1064 \mathrm{~nm}$ laser 
A Scanning Electron Microscope (SEM) Philips XL 30 with EDX detector (Philips, The Netherlands and EDAX, USA, respectively) was used for the imaging of the craters created by UV and IR ablation. The amount of mass ablated was calculated using the density and volume. Using a glass density of $2.5 \mathrm{~g} / \mathrm{cm}^{3}$, the volume was converted to nanograms removed.

The laser beams are focused perpendicular and parallel to the sample to create either single-pulse ablation or double-pulse prespark or plasma reheating schemes. The geometric configurations for double-pulse LIBS were optimized as follows; the orthogonal configuration included one beam perpendicular to the surface while the other pulse was parallel to the surface. During prespark experiment, the IR beam was focused $0.75 \mathrm{~mm}$ above the sample surface at $160 \mathrm{~mJ}$ creating a spark in air followed $7 \mu$ s later by the perpendicular UV ablation pulse at $29 \mathrm{~mJ}$. Figure 14 below demonstrates that at $7 \mu \mathrm{s}$ the greatest increase in intensity was observed.

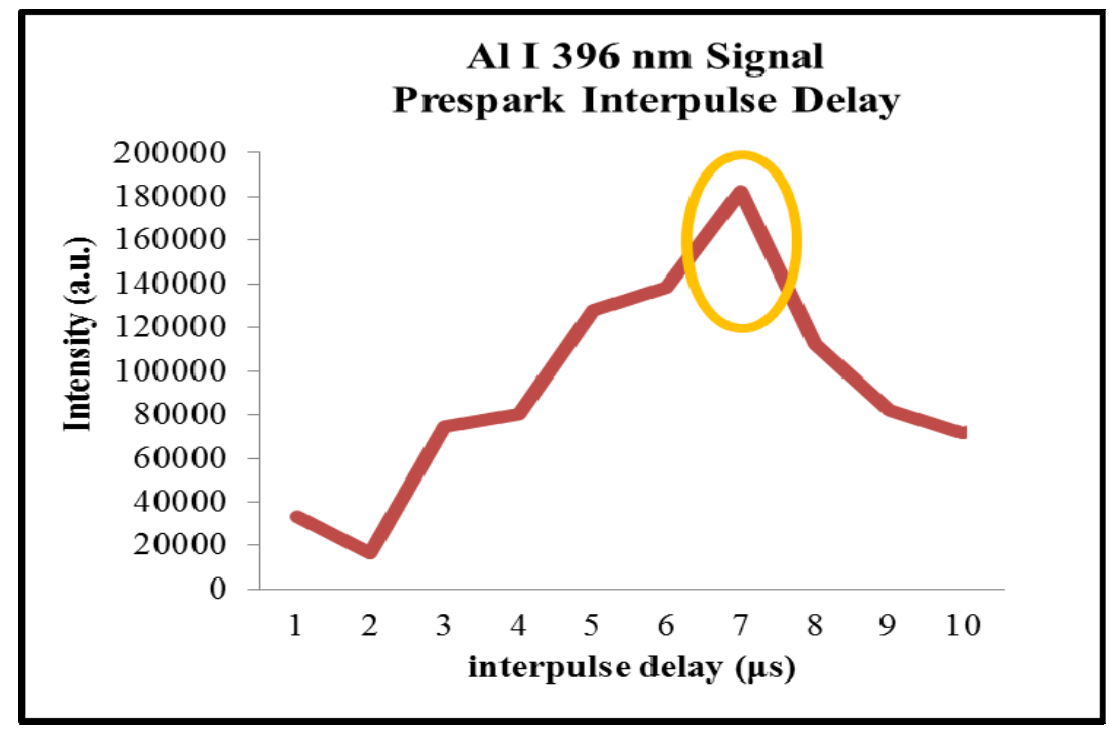

Figure 14. Optimized double-pulse LIBS prespark interpulse delay 
In the plasma reheat configuration, the UV ablation beam was pulsed at an energy of 27 $\mathrm{mJ}$, followed $400 \mathrm{~ns}$ later by a $35 \mathrm{~mJ}$ IR beam, focused $0.75 \mathrm{~mm}$ above the sample to reheat the plasma. Figure 15 shows the analytical signal during DP LIBS reheat to be at maximum signal with good precision at a delay of $400 \mathrm{~ns}$.

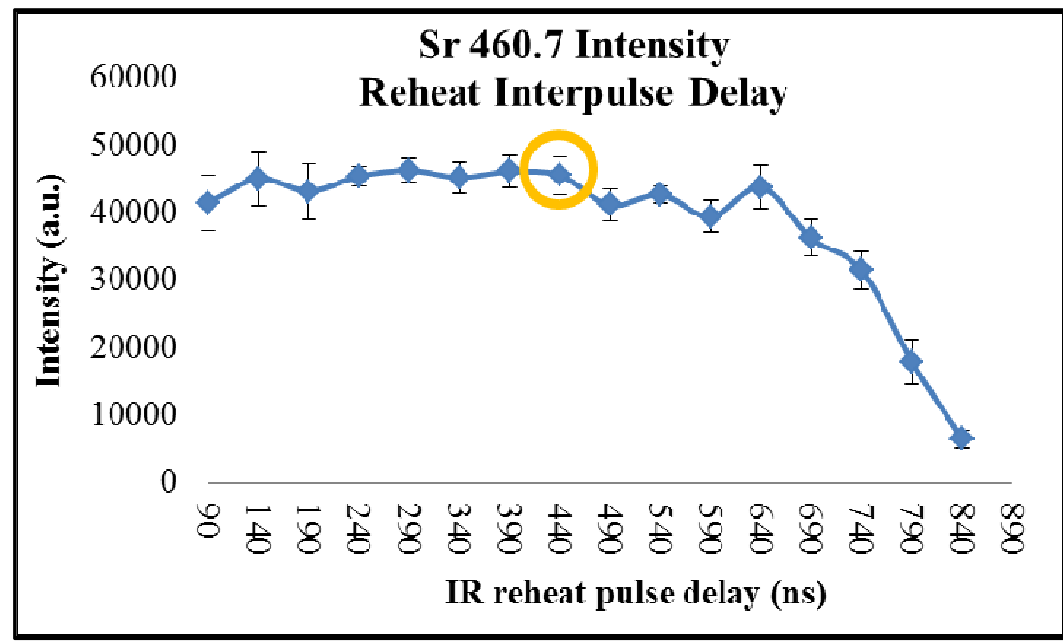

Figure 15. Optimized double-pulse LIBS reheat interpulse delay

Optical emissions from the plasma were acquired from the side $\left(90^{\circ}\right)$ by a pair of plano-convex lenses $(f=75 \mathrm{~mm})$ onto a fiber with a diameter of $50 \mu \mathrm{m}$ that was coupled to the entrance slit of an Andor Mechelle 5000 spectrometer equipped with an Andor iStar intensified CCD camera using a 1024 X 1024 chip. The optimized gate delays for both the $266 \mathrm{~nm}$ and $1064 \mathrm{~nm}$ experiments were $1.2 \mu \mathrm{s}$, while the optimized integration time was $3.5 \mu$ s and $11.0 \mu$ s for the $266 \mathrm{~nm}$ and the $1064 \mathrm{~nm}$ wavelengths, respectively. The broadband detector captured the spectral range between 200-950 $\mathrm{nm}$ with a resolution of $\sim 5000$. As a result of the broadband spectral analysis, the repetition rate for the spectrometer was $\sim 0.67 \mathrm{~Hz}$.

Analytical glass standards from National Institute of Standard and Technology (NIST) 614, 610, 612, 1831 and Bundeskriminalamt (BKA) glass reference standards 
FGS 01 and FGS 02 were used for development of the analytical protocols and to determine the precision, accuracy and repeatability of the LIBS analysis.

\subsection{Effects of Laser Wavelength}

The laser wavelength, pulse energy and irradiance are important properties and are responsible for producing a laser induced analytical plasma. The effect of laser wavelength influences the laser-material interaction as a result certain wavelengths will couple more efficiently with specific material and the plasma-material interaction. It has been documented that shorter wavelengths will produce increased photon energies which will aid in the ionization process ${ }^{70}$. For example, the $193 \mathrm{~nm}$ wavelength laser has a photon energy of $6.4 \mathrm{eV}$ compared to only $1.15 \mathrm{eV}$ for the $1064 \mathrm{~nm}$ laser. With sufficient photon energy, the ablation process will occur by photon ionization and non-thermal mechanisms ${ }^{70}$.

The plasma is produced by sample surface vaporization and absorption of the laser energy in the ablated material. The amount of ablated mass as a result of materialnanosecond laser interactions has been shown to vary significantly with the use of high power nanosecond laser irradiation ${ }^{149}$. The varation is a result of the high flux density and the ablated material being heat by the tailing end of the laser light.

Commercial laser ablation systems are now equipped with shorter wavelengths, i.e. $193 \mathrm{~nm}, 213 \mathrm{~nm}$ or $266 \mathrm{~nm}$ because it has been shown that UV wavelengths provide

more laser energy per unit volume for ablation, resulting in increased mass removal ${ }^{150}$ compared to IR ablation, better crater morphology ${ }^{31}$ and improved reproducibility of 
signal $^{31}$, demonstrating that the shorter wavelengths provide a more controlled ablation rate $^{30,150,150}$.

Several researchers have reported results from the use of different wavelengths ranging from the plasma characteristics such as temperature and density ${ }^{30}$, plasma formation $^{151}$, fractionation ${ }^{150}$, laser-sample coupling ${ }^{150,152}$, and quantification of analytical

results $^{30,153}$. The coupling of the laser energy to the sample is clearly affected by the irradiation wavelength. Previous studies have shown that the UV wavelength can improve the coupling efficiency when compared to the longer wavelengths, i.e., $532 \mathrm{~nm}$ and $1064 \mathrm{~nm}^{30,150}$.

\subsubsection{Crater Morphology}

Crater morphology influences the analytical results. LIBS on solid surfaces is dependent of the material ablated into the plasma plume ${ }^{72}$. LTSD is a critical parameter for LIBS measurements. A change in the depth of focus can affect the LIBS signal and reproducibility. Experimental trials demonstrated that by defocusing the beam, forcing the laser to focus into the surface, the reproducibility of the LIBS emission signals improved. The experimental trials determined that focusing the 1064 irradiance laser into the surface prevented shattering the glass, see Figure 16. The optimal LTSD determined for both the $1064 \mathrm{~nm}$ and $266 \mathrm{~nm}$ wavelengths were $0.50 \mathrm{~mm}$ and $1.30 \mathrm{~mm}$ focused into the sample, respectively.

The amount of mass ablated per laser pulse per unit area is defined as the ablation rate. LIBS typically removes mass from the sample in the ng- $\mu \mathrm{g}$ range, classifying it as a surface sampling technique. However, multiple pulses at the same location can be used 
to progressively ablate the surface, thereby removing more material and permitting depth profiling or bulk analysis of the sample. Researchers have reported that the $266 \mathrm{~nm}$ ablation removes more mass than the $532 \mathrm{~nm}$ or $1064 \mathrm{~nm}^{30,150,152,153}$ ablation experiments, which is a result of the better energy coupling efficiency provided by the UV, combined with less plasma shielding.

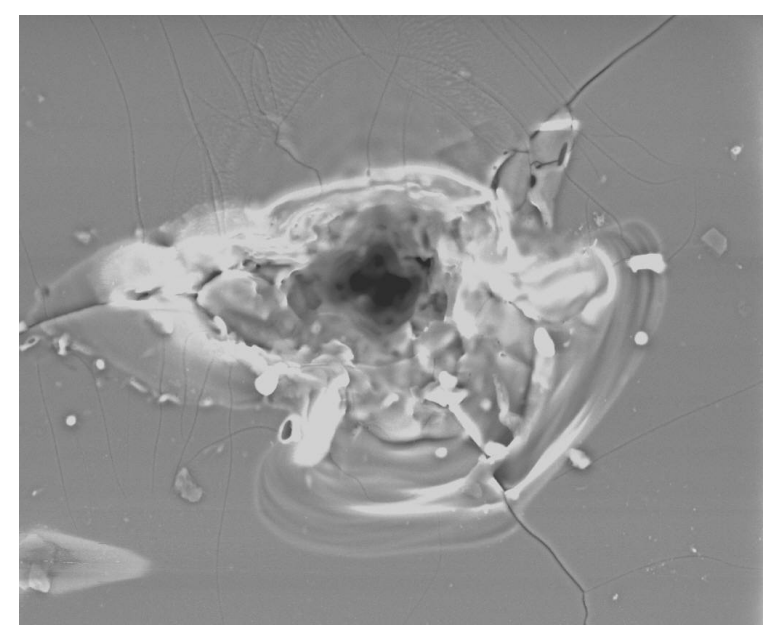

Figure 16. The damage caused to glass using IR $1064 \mathrm{~nm}$ laser

The crater diameter has been proven independent of the sample matrix, while the crater depth is dependent on sample matrix ${ }^{154}$. Previous conducted research has theorized that more mass ablated with minimal sample heating can provide a more stoichiometric sampling ${ }^{150}$. Analytical research has been conducted demonstrating that the IR plasma is hotter $^{155}$, which will increase the plasma shielding and reduce the amount of the IR energy that can be transferred to the sample surface ${ }^{156}$.

The crater morphology can be appreciated at a high 1000 times magnification as shown in Figure 17, demonstrating the differences for 100 shot ablations from the 266 nm ablations (Fig. 17a) compared to the $1064 \mathrm{~nm}$ ablations (Fig. 17b). Crater morphology produced at these lens-to-sample-distance (LTSDs) resulted in a diameter of 
$75 \mu \mathrm{m}$ and depth of $70 \mu \mathrm{m}$ for the $266 \mathrm{~nm}$ laser and a diameter of $37 \mu \mathrm{m}$ and depth of 26 $\mu \mathrm{m}$ for the $1064 \mathrm{~nm}$ laser. The total mass removed by the $266 \mathrm{~nm}$ ablation was found to be $\sim 790 \mathrm{ng}$, approximately ten times higher than the $81 \mathrm{ng}$ of the $1064 \mathrm{~nm}$ laser. The data acquired supports the theory that UV wavelengths, as a result their higher photonic energy, provide photoionization and/or multiphoton ionization.
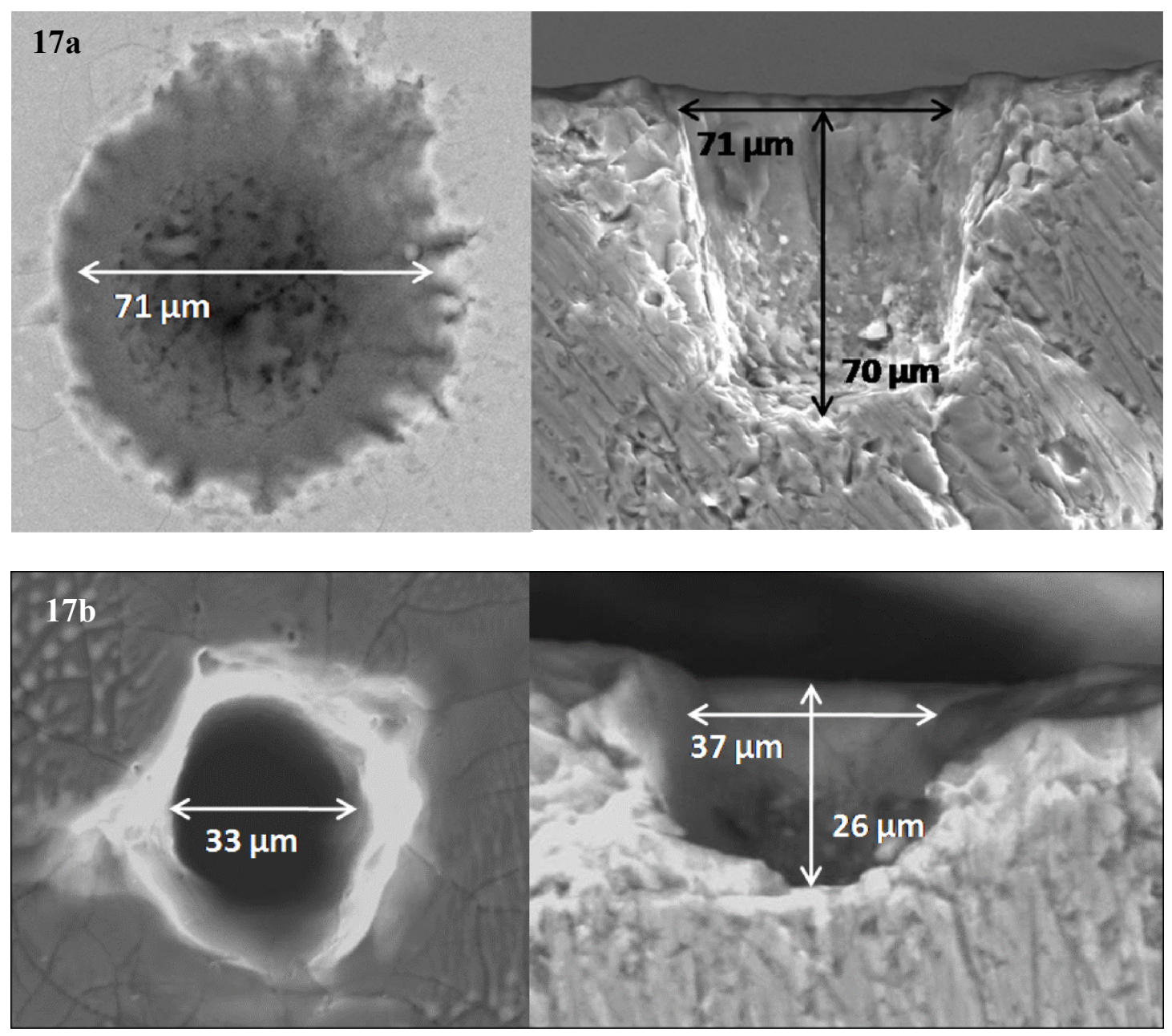

Figure 17. a) Crater formed using a $266 \mathrm{~nm}$ laser on NIST glass standard 1831 b) Crater formed using a 1064 nm laser on NIST glass standard 1831

Research studies determined that the differences of the mass removed between the single and double-pulse LIBS ablations was negligible; this is in agreement with previous 
research conducted by Santagata et $a l^{157}$. However, this contradicts research reported by Scaffidi et al., where they reported an eight to ten fold increase in mass ablated using the orthogonal double-pulse configuration ${ }^{101}$. Investigation of the crater morphology also reveals a more uniform crater produce by the $266 \mathrm{~nm}$ laser, which may translate to better precision in LIBS measurements. 


\subsection{Quantitative Analysis}

Quantitative analysis by LIBS is not standardized and many quantification methods have been proposed for chemical analyses. One of the key reasons for the proposal of different methods is because of the lack of standard reference materials. However, the availability of glass standard reference materials permits calibration curves for the quantitative chemical analyses and calibration curves have been proven effective for many years.

Construction of the calibration curves were produced by the line intensities (max counts of intensity minus the background) in correlation to the corresponding elemental concentration of the glass standard. Each point on the calibration curves represents an average of five replicates, with the second 50 of 100 shots being averaged. The vertical error bars are represented as \pm 1 standard deviation calculated from the 5 replicates. The calibration curves of the different atomic lines of the same element were very reproducible (see the error bars in figures 19a-19f) and were utilized for the quantification of trace elements in glass. All chosen spectral lines have minimal spectral interferences. LIBS produced sharp emission lines allowing for qualitative and quantitative analysis. Spectral peaks for emission lines Ba II 493 nm (32 ppm), K I 766 nm (2738 ppm), and Sr II $421 \mathrm{~nm}(89 \mathrm{ppm})$ can be seen in Figure 18 below for the different LIBS configurations of SP UV LIBS, SP IR LIBS and DP UV ablation IR reheat. 

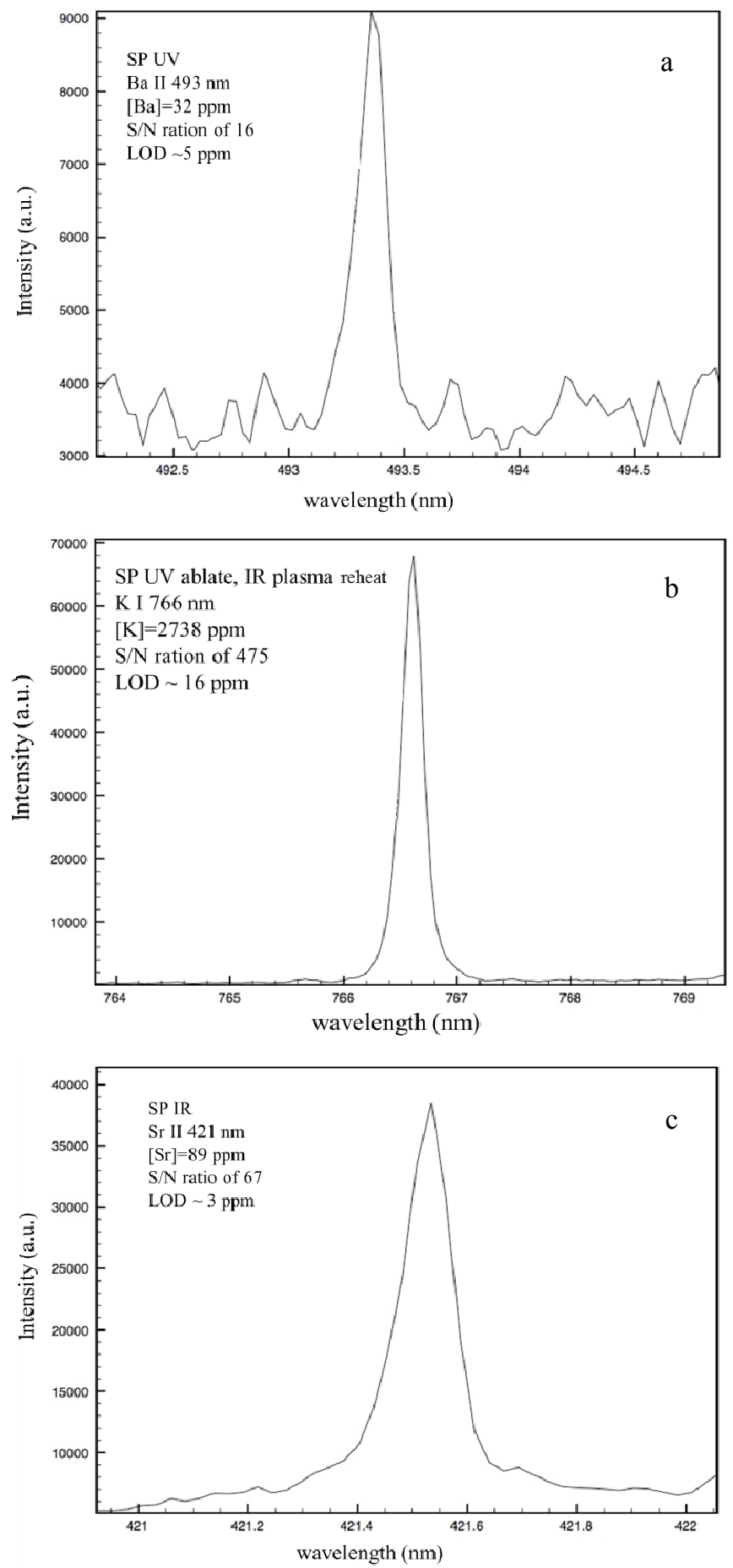

Figure 18. Spectral emission lines for a) Ba II 493.4 nm (32 ppm), b) K I 766.5 nm (2738 ppm) and c) Sr II 421 nm (89 ppm) 
The five glass standard reference materials and their corresponding concentrations are listed in Table 1. NIST glass standard 1831 is a float glass formulation and was analyzed twice for this study, with the second set of measurements treated as an "unknown" sample for comparison purposes.

Table 1. Certified Concentration of Glass Standards $(\mu \mathrm{g} / \mathrm{g})$

\begin{tabular}{|c|c|c|c|c|c|c|}
\hline \multirow[b]{2}{*}{ CRM } & \multicolumn{5}{|c|}{$\begin{array}{l}\text { Element Concentrations for Glass Reference Materials } \\
\qquad(\mu \mathrm{g} / \mathrm{g})\end{array}$} & \multirow[b]{2}{*}{$\mathrm{Ti}$} \\
\hline & $\mathrm{Sr}$ & $\mathrm{Ba}$ & $\mathrm{Al}$ & K & $\mathrm{Mg}$ & \\
\hline 1831 & 89 & 32 & 6381 & 2738 & 2116 & 114 \\
\hline 610 & 497 & 424 & 10006 & 486 & 465 & 434 \\
\hline 612 & 76 & 38 & 11165 & 66 & 77 & 48 \\
\hline FGS 1 & 57 & 40 & 1500 & 920 & 23900 & 69 \\
\hline FGS 2 & 253 & 199 & 7400 & 4600 & 23400 & 326 \\
\hline
\end{tabular}

Limits of detection (LOD) and limits of quantification (LOQ) were calculated according to $\mathrm{LOD}=3 \mathrm{~s}_{\mathrm{B}}$ and $\mathrm{LOQ}=10 \mathrm{~s}_{\mathrm{B}}$, where $\mathrm{s}_{\mathrm{B}}$ is the standard deviation of the background, which is taken as close to the emission line as possible without encountering spectral interferences. Trace elements K I 766.49 nm, Ba II $493.41 \mathrm{~nm}$, Sr II $407.77 \mathrm{~nm}$ and Ti II $336.12 \mathrm{~nm}$, were chosen as a result of previous work by our group, demonstrating the high discriminating power of these elements in the glass matrix ${ }^{3}$.

\subsubsection{Single-Pulse Quantitative Analysis}

Single-pulse LIBS spectra were acquired as previously described using both by $266 \mathrm{~nm}$ and $1064 \mathrm{~nm}$ lasers. The experimental conditions that provided the highest precision and 
accuracy were determined and used throughout the study.

The energy of the $266 \mathrm{~nm}$ laser was held constant at $29 \mathrm{~mJ}$, while the $1064 \mathrm{~nm}$ laser was held at $47 \mathrm{~mJ}$. It was found that at these energies the reproducibility of the experiments increased and limiting the IR wavelength to $47 \mathrm{~mJ}$ prevented cracking and damaging of the glass during ablation. A detector delay of $1.20 \mu$ s was used, which allowed for the decay of background continuum and production of sharp emission lines. Figure 19 illustrates calibration curves produced by single-pulse 266 and $1064 \mathrm{~nm}$. Each measurement corresponds to the intensity of the emission line of interest obtained with an accumulation on the detector of the second 50 accumulation of 100 laser shots. Singlepulse UV and IR configurations demonstrate good correlation with most $\mathrm{R}^{2}$ values being greater than 0.990. It has been demonstrated in previous research that IR irradiances show greater enhancement with ionic emission lines ${ }^{98}$, this is witnessed when comparing K I $766.5 \mathrm{~nm}$ and Sr II $407.7 \mathrm{~nm}$.

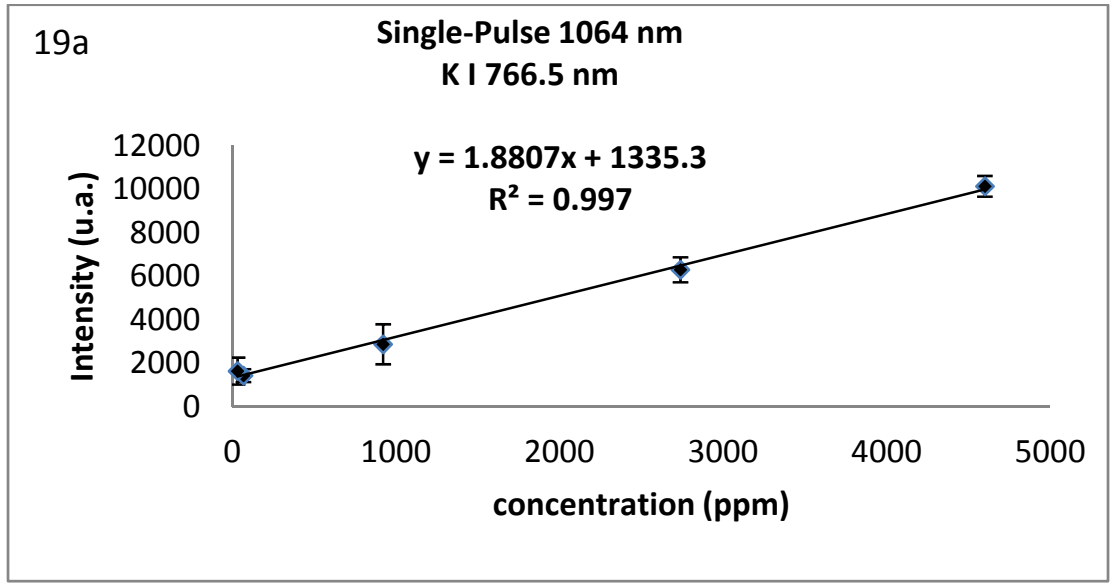



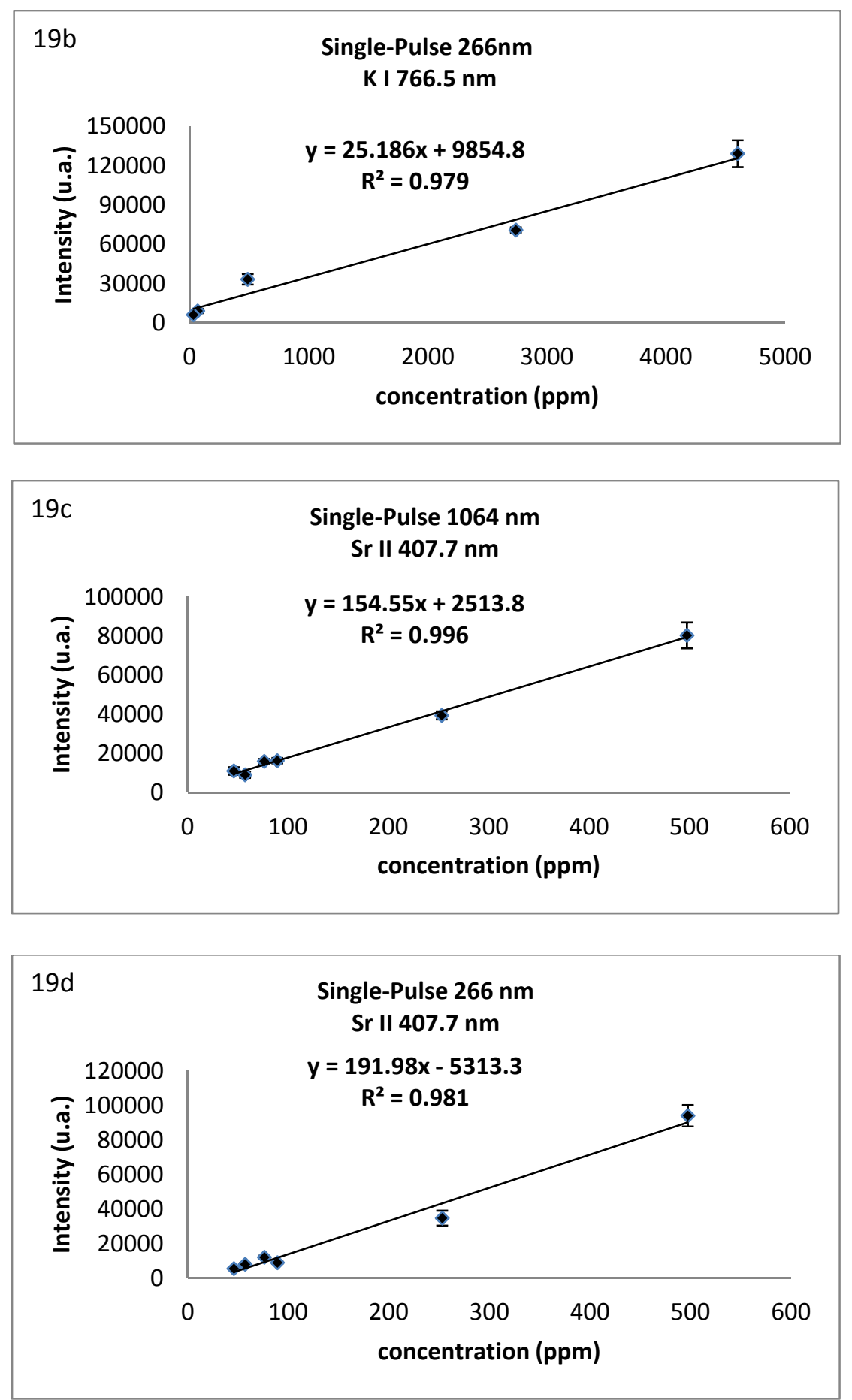

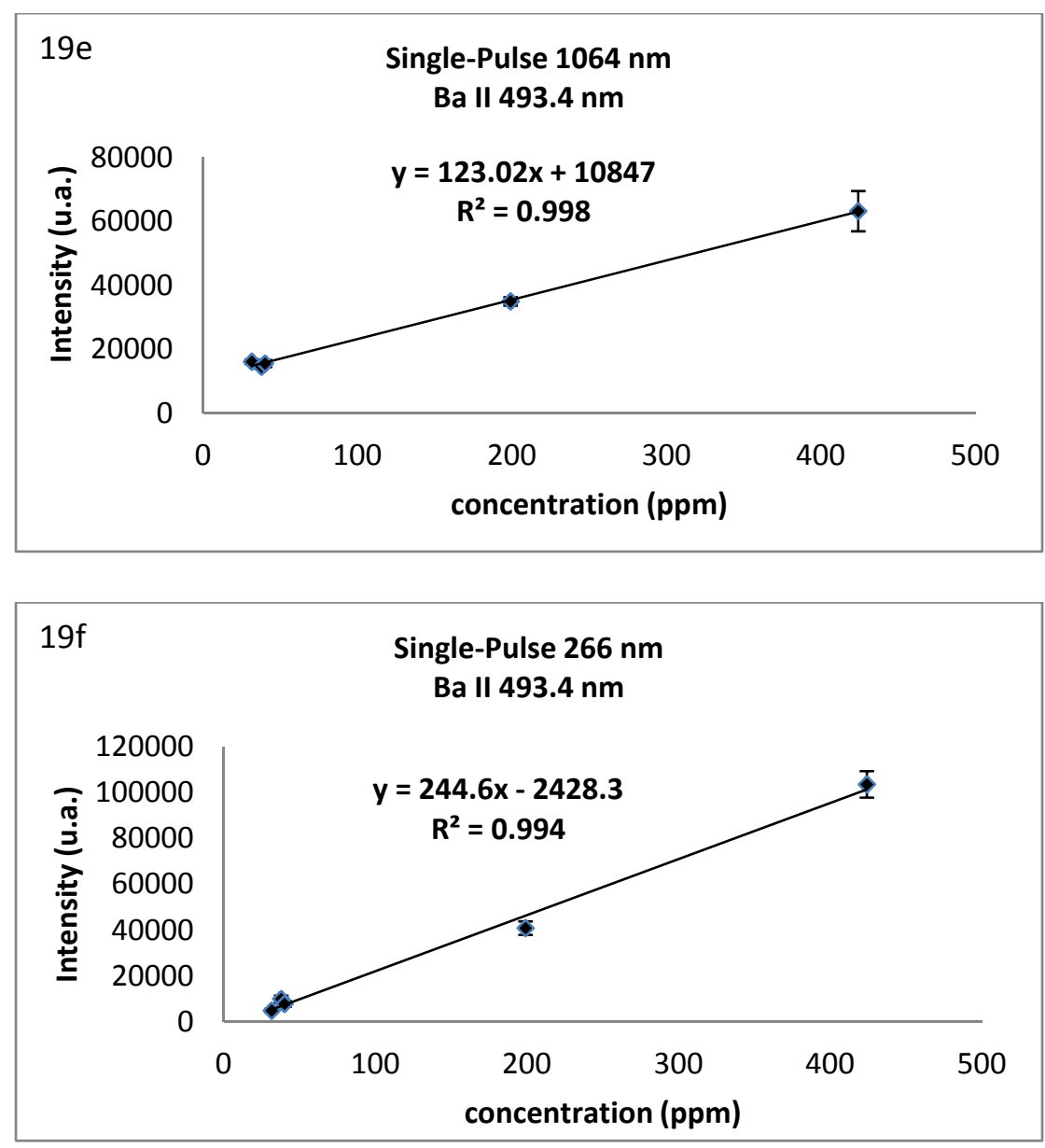

Figure 19. a) Single-pulse 1064 nm calibration curves for K I $766.5 \mathrm{~nm}$; b) Single-Pulse 266 nm calibration curve for K I $766.5 \mathrm{~nm}$; c) Single-pulse $1064 \mathrm{~nm}$ calibration curves for Sr II 407.7; d) Single-Pulse $266 \mathrm{~nm}$ calibration curve for Sr II 407.7; e) Single-pulse $1064 \mathrm{~nm}$ calibration curves for Ba II 493.4; f) Single-Pulse 266 nm calibration curve for Ba II 493.4

As mentioned earlier in this paper, NIST 1831 standard was chosen and analyzed twice, the second time being treated as an "unknown" sample. As both methods show good correlation, the UV irradiance demonstrated better precision for the K and Sr lines. The IR irradiance proved to have lower limits of detection, however, in trace elemental analysis of glass, the LOD obtained by UV LIBS ablation would provide sufficient sensitivity for the chemical characterization of glass, see Table 2. 
Table 2. Figures of Merit for the Single-Pulse UV 266 nm (SP UV)

\begin{tabular}{ccccccc}
\hline Method & Sample & Peak (nm) & Precision (\%) & Bias (\%) & LOD (ppm) & LOQ (ppm) \\
\hline SP UV & 1831 & K I 766.5 & 8.23 & 12.15 & 5.93 & 19.77 \\
SP UV & 1831 & Sr II 407.7 & 5.80 & 16.82 & 4.10 & 13.68 \\
SP UV & 1831 & Ba II 493.4 & 10.83 & 9.48 & 2.25 & 7.51 \\
\hline
\end{tabular}

\subsubsection{Double-Pulse Quantitative Analysis}

Double-pulse LIBS has gained merit over the recent years. Improvements in the figures of merit by double-laser pulse configurations have contributed to better sensitivity and signal gain for LIBS analyses ${ }^{98,99}$. Several double-pulse geometric configuration approaches have been used consisting of either one laser $^{158}$, or two different lasers ${ }^{159}$ to encompass a double-pulse LIBS setup.

Orthogonal pre-spark and plasma reheat were the two configurations used during this work. Optimal separation time between the two laser pulses was determined by plotting the LIBS emission intensity versus the delay time. The main objective of this study was not given to the highest intensity enhancement, but rather to increased precision and accuracy. Demonstrated in this study, an intensity decrease is seen from single-pulse to double-pulse orientation. The decrease in signal was also reported by Gautier $e t a l .{ }^{98}$, when they observed lower line emissions using UV ablation, from elements with lower excitation energies of approximately $4 \mathrm{eV}$, in the double-pulse reheating approach when compared to single-pulse. The elements of interest investigated here all have lower excitation energies of 1.6, 3.0, and $2.5 \mathrm{eV}$ for K I $766.5 \mathrm{~nm}$, Sr II $407.7 \mathrm{~nm}$, and Ba II $493.4 \mathrm{~nm}$, respectively, which could account for the witnessed decrease in intensity. 
Double-pulse plasma reheat has been attributed to higher plasma temperatures and electron densities because of the larger plasma size after the second plasma reheat beam, which would allow for longer integration times. As can be seen by Figure 20, all correlation coefficients are greater than 0.990 , demonstrating very good linearity. The precision demonstrated by the plasma reheat scheme is moderately lower than that by single-pulse UV, which has also been reported by Scaffidi et al. ${ }^{160}$, and comparable to that of single-pulse IR configuration. As emission enhancement is not seen, however, most LODs have decreased. The LOD for K I $766.5 \mathrm{~nm}$ and Sr II $407.7 \mathrm{~nm}$ have decreased from the single-pulse value of 5.93 and $4.10 \mathrm{ppm}$ to 4.30 and $3.17 \mathrm{ppm}$, respectively, showing an increase in the double-pulse plasma reheat sensitivity, see Table 3.

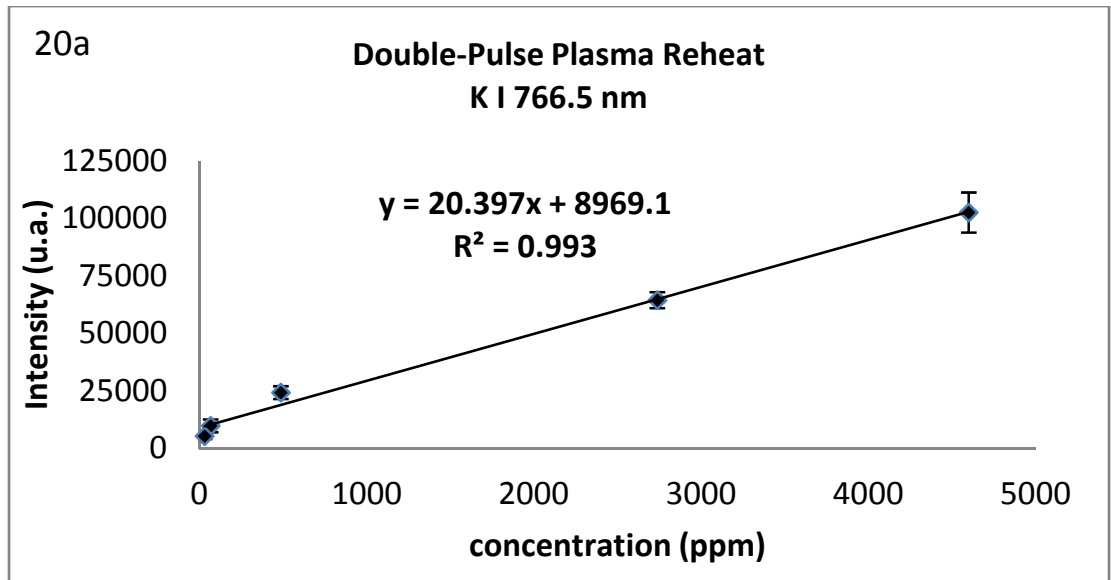




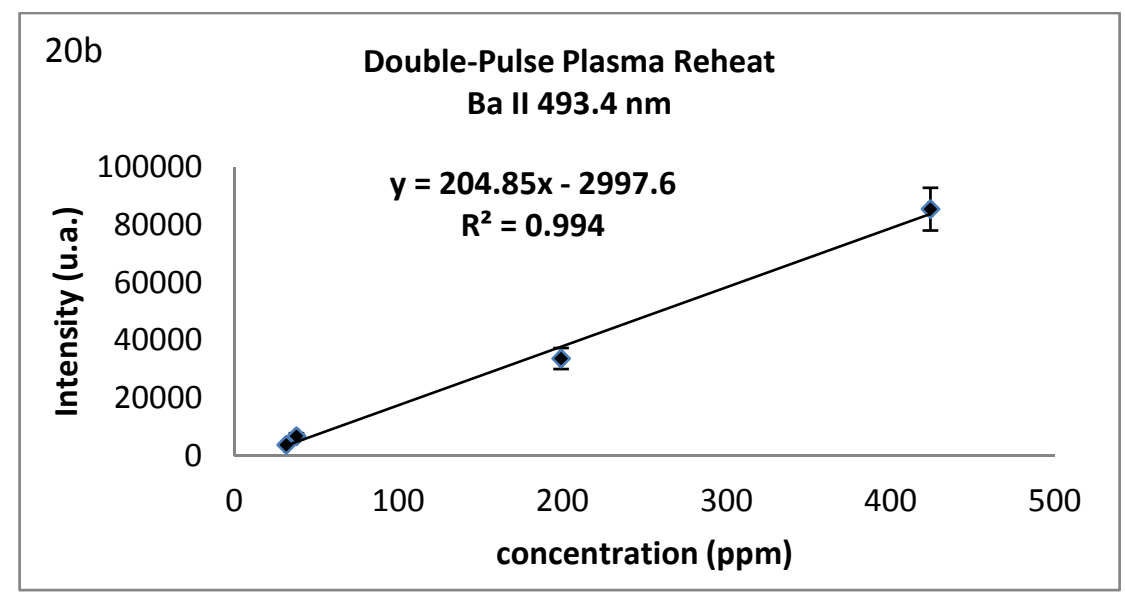

Figure 20. Calibration curves for double-pulse plasma reheat with $266 \mathrm{~nm}$ ablation for a) $\mathrm{K}$ I 766.5 nm and b) Ba II $493.4 \mathrm{~nm}$

Prespark LIBS is theorized to cause ambient gas breakdown before the ablation pulse is produced, creating different effects that could lead to an increased emission by the analyte. It was hypothesized that the reduction in ambient gas causes the plasma to expand faster, thereby reaching a larger size, while producing less plasma shielding for the ablation pulse ${ }^{106,161}$. Higher sensitivity was also evident in the double pulse prespark configuration, than when compared to all other experimental data, because of the resolution of emission line Ti II $336.12 \mathrm{~nm}$. Emission line Ti II $336.12 \mathrm{~nm}$ was pronounced and a linear correlation achieved, see Figure 21, while in the other configurations the Ti II line was not resolved well enough for calibration. As seen in the plasma reheat configuration the linearity between concentration and intensity proves well, achieving correlation coefficients most greater than 0.990 . However, the prespark shows less precision than that demonstrated by either the UV/IR single-pulse or the plasma reheat, see Table 3. 

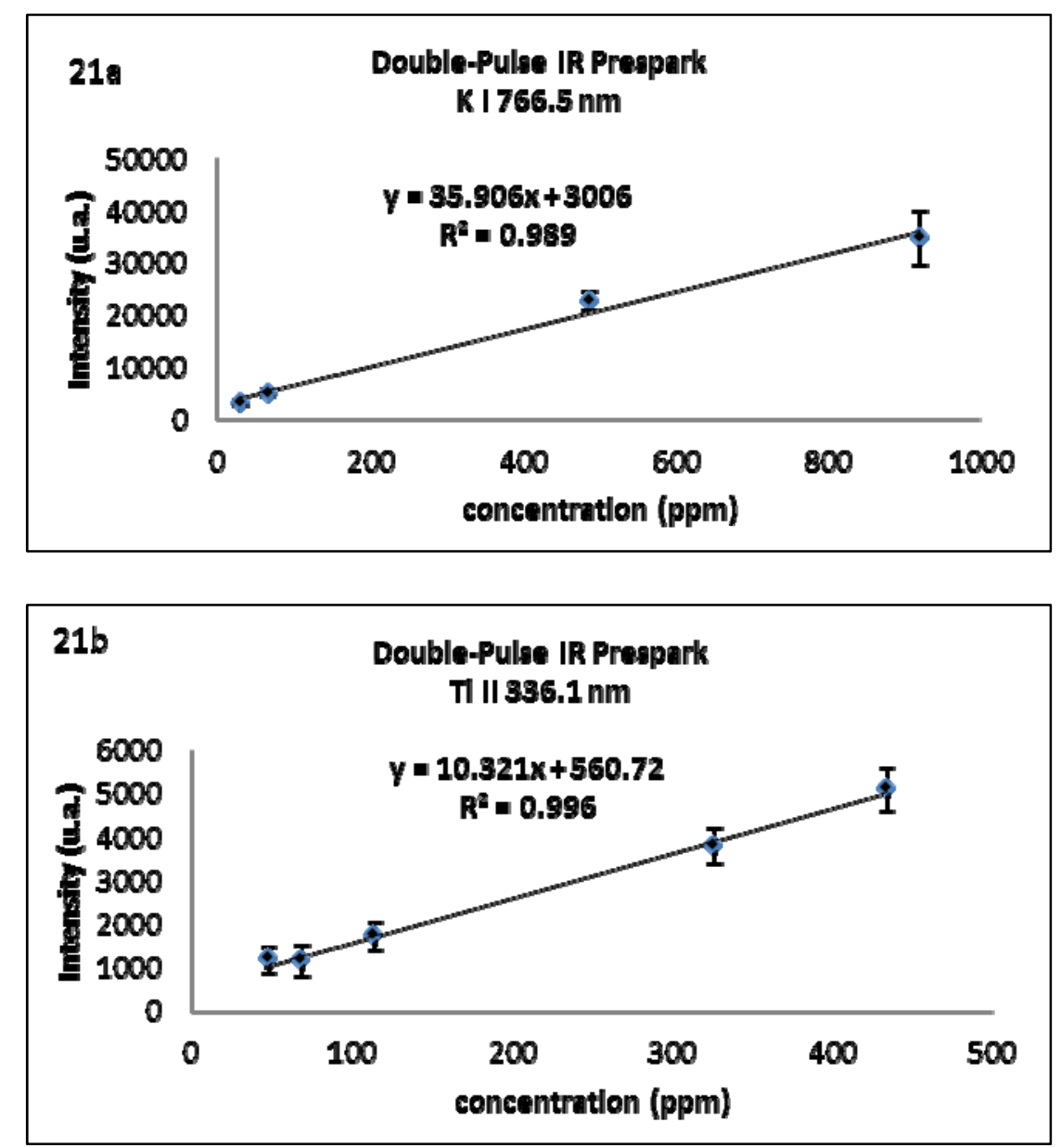

Figure 21. Calibration curves for double-pulse IR pre-spark with $266 \mathrm{~nm}$ ablation for a) $\mathrm{K}$ I 766.5 nm and b) Ti II $336.1 \mathrm{~nm}$

The reproducibility of each point in the calibration curve was $10-15 \%$. The accuracy of the analysis was evaluated to be $\sim 10 \%$. The determined limits of detection for metal ions in this work were certainly comparable, if not lower with those reported in other publications. Kurniawan et al. performed quantitative analysis on glass samples and reported the LODs for $\mathrm{Ba}, \mathrm{K}$, and Ti were 190 ppm, 190 ppm, and 410 ppm, respectively ${ }^{162}$. Ismail et al. reported the LOD of Ti to be $100 \mathrm{ppm}$ for single-pulse and $10 \mathrm{ppm}$ for double-pulse, Yamamoto et al. detected $\mathrm{Ba}$ and $\mathrm{Sr}$ in toxic soil and produced a LOD of 265 ppm and 42 ppm, respectively, and Cremers et al. detected Ba in soil to 
have an LOD of $26 \mathrm{ppm}^{163-165}$. This exhibits the evolution LIBS is making as a choice analytical technique in the forensic science community.

Table 3. LIBS Figures of Merit for Single-Pulse, Double-Pulse Reheat, Double-Pulse Prespark and Single-Pulse $1064 \mathrm{~nm}$ Laser

\begin{tabular}{ccccccc}
\hline Method & Sample & Peak (nm) & Precision (\%) & Bias (\%) & LOD (ppm) & LOQ (ppm) \\
\hline SP UV & 1831 & K I 766.5 & 8.23 & 12.15 & 5.93 & 19.77 \\
SP UV & 1831 & Sr II 407.7 & 5.80 & 16.82 & 4.10 & 13.68 \\
SP UV & 1831 & Ba II 493.4 & 10.83 & 9.48 & 2.25 & 7.51 \\
DP Reheat & 1831 & K I 766.5 & 3.61 & 14.82 & 4.30 & 14.34 \\
DP Reheat & 1831 & Sr II 407.7 & 8.43 & 5.89 & 3.17 & 10.56 \\
DP Reheat & 1831 & Ba II 493.4 & 12.38 & 19.95 & 1.72 & 5.73 \\
DP PS & 1831 & K I 766.5 & 10.45 & 19.06 & 3.77 & 12.58 \\
DP PS & 1831 & Sr II 407.7 & 30.38 & 47.74 & 3.02 & 10.07 \\
DP PS & 1831 & Ti II 336.1 & 31.67 & 42.13 & 11.03 & 36.77 \\
SP IR & 1831 & K I 766.5 & 9.13 & 16.50 & 4.07 & 13.56 \\
SP IR & 1831 & Sr II 407.7 & 8.26 & 8.98 & 3.28 & 10.92 \\
SP IR & 1831 & Ba II 493.4 & 4.51 & 39.68 & 1.98 & 6.59 \\
\hline
\end{tabular}




\subsection{Conclusions}

The LIBS technique has increasingly gained attention as a competitive analytical tool for surface analysis, depth profiling, and bulk analysis of solids. Laser wavelength and laser energy play an influential role in analytical performance for LIBS due to the lasermaterial interaction and the initiation of the plasma.

In the current work, correlation coefficients of $>0.990$ were achieved for calibration curves for the trace elements analyzed in the range between 32 and 4600 ppm. Precision and accuracy for the quantitative analysis of standards ranged from as good as $4.5 \% \mathrm{RSD}$ for the precision of glass at $32 \mathrm{ppm}$ for the measurement of $\mathrm{Ba}$ and an accuracy of $9.0 \%$ bias but more typically resulting in $9-10 \%$ RSD for the precision and $10 \%$ bias for most elements of interest in these glasses for both the UV and IR experiments and the single- and double-pulse experiments.

The IR ablations resulted in typically less emission intensities, an indication that less mass was ablated when IR was used. Less mass being ablated with the IR nm laser was corroborated with mass removal calculations as the ablation rate between the IR and UV produced significant differences. The plasma shielding was demonstrated to be less with UV irradiance and the laser-sample energy coupling was more efficient with the $\mathrm{UV}$, resulting in better precision and accuracy in most but not all experimental configurations. The UV irradiation produced a more uniform crater, translating to better precision from better signal reproducibility. The craters formed resulting from IR irradiance, even when higher power than UV was used, resulted in less mass ablated and severe cracking and damage to the sample surface, in comparison to the UV. 
The LODs for the elements of interest in forensic glass analysis were adequate for the proper characterization and comparison of glass using any of the UV and IR configurations performed in this study. The same emission lines where used in the comparisons for all configurations and, under these comparison conditions, it was concluded that the use of $266 \mathrm{~nm}$ irradiation was recommended for the forensic analysis and comparison of glass with single-pulse experiments providing very good analytical data and minimal sample damage. 


\section{Discrimination and Association of Float Glass by Laser Induced Breakdown Spectroscopy from Single Manufacturing Plants}

Laser induced breakdown spectroscopy is an emerging analytical technique for the forensic association and discrimination of glass. The emission intensities are utilized in LIBS in the ultraviolet, visible and near infrared spectrum. The LOD for LIBS will vary with each element but it has been documented to achieve LODs as low as $\sim 2$ ppm for the Ba II $493.4 \mathrm{~nm}$ and Sr II $407.7 \mathrm{~nm}$, and 4 ppm for the K I $766.5 \mathrm{~nm}$ emission line ${ }^{31}$.

These LODs are sufficient for the forensic analysis of glass. The LIBS research

conducted to date has been successful in the forensic analysis of glass ${ }^{3,13,28,42}$. Naes et al. reported that LIBS produces the same $99 \%$ discrimination, with no false exclusions, as $\mu \mathrm{XRF}$ and LA-ICP-MS with a different element menu of automobile float glass ${ }^{3}$. Bridge et al. achieved an $83 \%$ and $74 \%$ discrimination of automobile float glass with LIBS, however when combined with refractive index measurements, the discrimination increased to $99 \%$ discrimination for both sample sets ${ }^{13,42}$. The precision obtained from both authors were in agreement, Naes et al. states the precision less than 10\% RSD and Bridge et al. states an average precision of 7\% RSD.

The advantages offered by LIBS include a sensitive and fast approach to elemental analysis and LIBS permits small sample size with good precision, similar to LA-ICP-MS. The instrumentation of LIBS is fairly inexpensive when compared to the more established techniques of LA-ICP-MS, LA-ICP-OES and $\mu$ XRF, it is less complex, generates data virtually instantaneously, has the capability to be portable, while providing 
high sample throughput. Common disadvantages often described are shot-to-shot irreproducibility, high continuum background, line broadening and self-absorption.

Float glass lines are designed to operate 365 days a year. Typically, 300-600 tons of glass per day are produced in float glass furnaces of which mostly is for architectural and automotive glass products ${ }^{20}$. To date, however, no studies have been reported presenting LIBS for the association and discrimination of float glass from a single plant over periods of time. This chapter focuses on the capability for LIBS to discriminate and associate float glass produced at the same plant at approximately the same time period, i.e., from days apart to years apart.

\subsection{Float Glass Sample Descriptions}

All samples were examined on the non-float surfaces of the respective glass. National Institute of Standards and Technology (NIST) certified reference material (CRM) float glass 1831 was included into the experimental procedure to evaluate bias, precision and accuracy, due to the similar matrix. Duplicate samples were analyzed repeatedly throughout experimental procedure as a positive control for method validation to assess type I error. Float glass samples ranging from days apart to years apart were collected from manufacturing plants in the United States to determine the capability of LIBS for forensic glass analysis from single manufacturing plants.

\subsubsection{Sample Set 1: Cardinal Glass Industries}

Sample set one is composed of 49 colorless float glass samples manufactured in Cardinal Glass Industries (Portage, WI, USA) from May 1997 to September 2001. All the 
fragments were clear float glasses that ranged in refractive index from $1.51817-$ 1.51905. Table 2 summarizes sample set one.

Table 4. Sample Set 1, Cardinal Glass Industries

\begin{tabular}{|c|c|c|c|}
\hline Source & Sample Description & Color & RI \\
\hline Cardinal & $5 / 16 / 1997$ & Clear & 1.51848 \\
\hline Cardinal & $7 / 15 / 1997$ & Clear & 1.51817 \\
\hline Cardinal & $7 / 18 / 1997$ & Clear & 1.51841 \\
\hline Cardinal & $8 / 17 / 1997$ & Clear & 1.51840 \\
\hline Cardinal & $9 / 16 / 1997$ & Clear & 1.51837 \\
\hline Cardinal & $10 / 15 / 1997$ & Clear & 1.51838 \\
\hline Cardinal & $11 / 15 / 1997$ & Clear & 1.51840 \\
\hline Cardinal & $12 / 15 / 1997$ & Clear & 1.51841 \\
\hline Cardinal & $1 / 14 / 1998$ & Clear & 1.51835 \\
\hline Cardinal & $2 / 15 / 1998$ & Clear & 1.51817 \\
\hline Cardinal & $4 / 15 / 1998$ & Clear & 1.51878 \\
\hline Cardinal & $5 / 17 / 1998$ & Clear & 1.51897 \\
\hline Cardinal & $6 / 14 / 1998$ & Clear & 1.51880 \\
\hline Cardinal & $7 / 17 / 1998$ & Clear & 1.51870 \\
\hline Cardinal & $8 / 12 / 1998$ & Clear & 1.51869 \\
\hline Cardinal & $9 / 20 / 1998$ & Clear & 1.51881 \\
\hline Cardinal & $11 / 14 / 1998$ & Clear & 1.51902 \\
\hline Cardinal & $1 / 15 / 1999$ & Clear & 1.51882 \\
\hline Cardinal & $3 / 15 / 1999$ & Clear & 1.51901 \\
\hline Cardinal & $4 / 14 / 1999$ & Clear & 1.51894 \\
\hline Cardinal & $5 / 20 / 1999$ & Clear & 1.51895 \\
\hline Cardinal & $6 / 11 / 1999$ & Clear & 1.51905 \\
\hline Cardinal & $7 / 10 / 1999$ & Clear & 1.51898 \\
\hline Cardinal & $8 / 19 / 1999$ & Clear & 1.51895 \\
\hline Cardinal & $9 / 14 / 1999$ & Clear & 1.51884 \\
\hline Cardinal & $10 / 18 / 1999$ & Clear & 1.51902 \\
\hline Cardinal & $11 / 24 / 1999$ & Clear & 1.51889 \\
\hline Cardinal & $12 / 13 / 1999$ & Clear & 1.51878 \\
\hline Cardinal & $1 / 1 / 2000$ & Clear & 1.51887 \\
\hline Cardinal & $1 / 18 / 2000$ & Clear & 1.51879 \\
\hline Cardinal & $2 / 1 / 2000$ & Clear & 1.51865 \\
\hline Cardinal & $2 / 24 / 2000$ & Clear & 1.51892 \\
\hline Cardinal & $3 / 1 / 2000$ & Clear & 1.51890 \\
\hline Cardinal & $3 / 27 / 2000$ & Clear & 1.51877 \\
\hline Cardinal & $4 / 1 / 2000$ & Clear & 1.51878 \\
\hline Cardinal & $4 / 16 / 2000$ & Clear & 1.51882 \\
\hline
\end{tabular}




\begin{tabular}{|c|c|l|c|}
\hline Source & Sample Description & Color & RI \\
\hline Cardinal & $5 / 1 / 2000$ & Clear & 1.51876 \\
\hline Cardinal & $6 / 1 / 2000$ & Clear & 1.51875 \\
\hline Cardinal & $10 / 1 / 2000$ & Clear & 1.51870 \\
\hline Cardinal & $11 / 1 / 2000$ & Clear & 1.51862 \\
\hline Cardinal & $12 / 1 / 2000$ & Clear & 1.51874 \\
\hline Cardinal & $1 / 1 / 2001$ & Clear & 1.51880 \\
\hline Cardinal & $2 / 1 / 2001$ & Clear & 1.51886 \\
\hline Cardinal & $3 / 1 / 2001$ & Clear & 1.51866 \\
\hline Cardinal & $4 / 1 / 2001$ & Clear & 1.51870 \\
\hline Cardinal & $5 / 1 / 2001$ & Clear & 1.51875 \\
\hline Cardinal & $6 / 1 / 2001$ & Clear & 1.51885 \\
\hline Cardinal & $7 / 1 / 2001$ & Clear & 1.51857 \\
\hline Cardinal & $8 / 1 / 2001$ & Clear & 1.51876 \\
\hline
\end{tabular}




\subsubsection{Sample Set 2: Pilkington North America, Inc.}

Sample set two is composed of 27 colorless float glass samples collected from September 2008 to April 2010. These samples were manufactured in Pilkington North America Inc. (Stockton, CA, USA). This plant underwent an Fe transition from high to low concentration from March 14, 2010 to March 19, 2010. The refractive indices of these samples were not determined as a result of previous research demonstrating the narrow spread of data. Table 3 summarizes sample set two.

Table 5. Sample Set 2, Pilkington North America, Inc.

\begin{tabular}{|c|c|c|}
\hline Source & Sample Description & Color \\
\hline Pilkington & $9 / 24 / 08$ & Clear \\
\hline Pilkington & $10 / 12 / 08$ & Clear \\
\hline Pilkington & $12 / 28 / 08$ & Clear \\
\hline Pilkington & $12 / 26 / 08$ & Clear \\
\hline Pilkington & $1 / 4 / 09 \mathrm{~B}$ & Clear \\
\hline Pilkington & $1 / 4 / 09$ & Clear \\
\hline Pilkington & $4 / 26 / 09$ & Clear \\
\hline Pilkington & $6 / 16 / 09$ & Clear \\
\hline Pilkington & $7 / 24 / 09$ & Clear \\
\hline Pilkington & $8 / 17 / 09$ & Clear \\
\hline Pilkington & $9 / 06 / 09$ & Clear \\
\hline Pilkington & $10 / 4 / 09$ & Clear \\
\hline Pilkington & $11 / 15 / 09$ & Clear \\
\hline Pilkington & $12 / 19 / 09$ & Clear \\
\hline Pilkington & $1 / 24 / 10$ & Clear \\
\hline Pilkington & $1 / 31 / 10$ & Clear \\
\hline Pilkington & $2 / 7 / 10$ & Clear \\
\hline Pilkington & $2 / 14 / 10$ & Clear \\
\hline Pilkington & $2 / 18 / 10$ & Clear \\
\hline Pilkington & $2 / 25 / 10$ & Clear \\
\hline Pilkington & $3 / 3 / 10$ & Clear \\
\hline Pilkington & $3 / 14 / 10$ & Clear \\
\hline Pilkington & $3 / 19 / 10$ & Clear \\
\hline Pilkington & $3 / 26 / 10$ & Clear \\
\hline Pilkington & $4 / 2 / 10$ & Clear \\
\hline Pilkington & $4 / 16 / 10$ & Clear \\
\hline Pilkington & $4 / 9 / 10$ & Clear \\
\hline
\end{tabular}




\subsection{Experimental Instrumentation and Procedure}

The LIBS experiments were conducted on a home-built LIBS system. The system consisted of a New Wave Research Q-switched Nd:YAG Tempest laser (New Wave Research, Fremont, CA) operating at $266 \mathrm{~nm}$, with a pulse width of $5 \mathrm{~ns}$. Flashlamps and Q-switches were both externally triggered using a Berkeley Nucleonics delay generator (San Rafael, CA). The laser beam was expanded from $\sim 4 \mathrm{~mm}$ to $12 \mathrm{~mm}$ using a Galilean telescope and focused at the normal incidence with a plano-convex lens with a focal distance of $150 \mathrm{~mm}$. The laser remained at a constant energy of $27 \mathrm{~mJ}$ per laser pulse, producing a spot size of $\sim 80 \mu \mathrm{m}$. All analyses were conducted at atmospheric pressure with an argon sheath being delivered over the sample surface at $900 \mathrm{ml} / \mathrm{min}$. Figure 22 displays a simple schematic of the LIBS system.

The plasma emissions were acquired at a $90^{\circ}$ angle from the side using a pair of plano-convex lenses with a focal distance of $75 \mathrm{~mm}$ onto a $50 \mu \mathrm{m}$ diameter fiber optic cable. The fiber optic cable was coupled to an Andor Mechelle 5000 spectrometer equipped with an intensified CCD camera using a 1024 x 1024 chip. The collected spectral range was from $200-950 \mathrm{~nm}$ with a resolution of $\sim 5000$. The repetition rate for the spectrometer was $0.67 \mathrm{~Hz}$.

To ensure representative sampling and to account for inherent glass heterogeneity, each glass sample was analyzed in replicates of five at different locations. Each replicate was the result of an accumulation of the last 50 laser pulses from a total 100 pulse replicates. To achieve the best reproducibility, highest signal-to-noise ratio, precision and accuracy, the lens-to-sample distance (LTSD), gate delay and integration time were 
optimized. The laser was focused $1.3 \mathrm{~mm}$ into the sample surface, the optimized gate delay with respect to the laser pulse was $1.5 \mu$ s and the integration width was $12.0 \mu \mathrm{s}$.

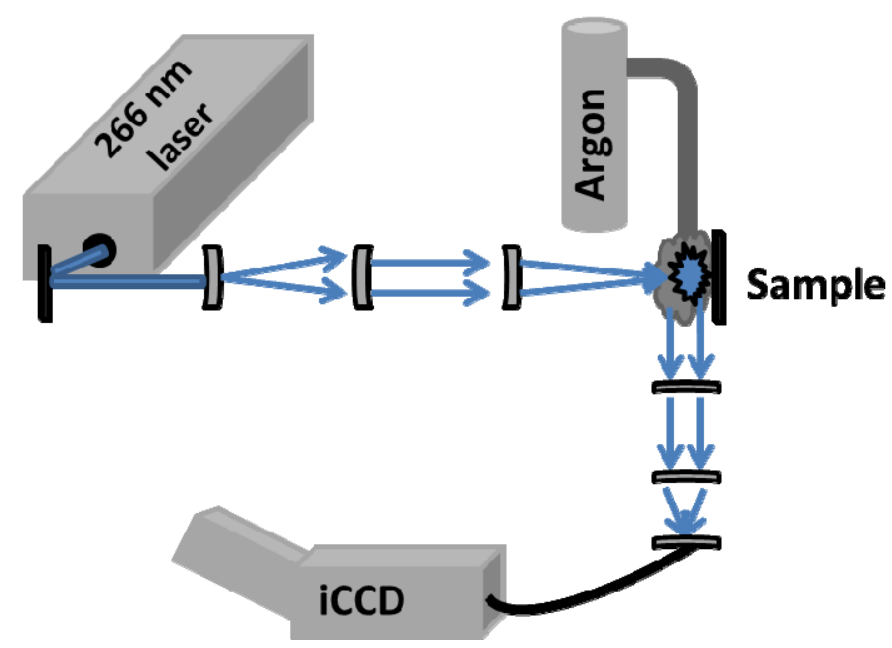

Figure 22. LIBS experimental setup schematic for forensic glass discrimination

Eighteen (18) emission lines corresponding to elements, $\mathrm{Sr}, \mathrm{K}, \mathrm{Fe}, \mathrm{Ca}, \mathrm{Al}, \mathrm{Ba}, \mathrm{Na}$,

$\mathrm{Mg}$ and $\mathrm{Ti}$, were initially chosen because of their presence throughout the glass spectra.

A typical float glass spectrum is seen in Figure 23. All emission peaks were background subtracted and the intensities were used for statistical analysis. If the precision for the stated elemental intensities were less $10 \%$ relative standard deviations (RSD) determined by the five replicates, the emission line was kept with the possibility of later being used in an elemental ratio for discrimination. 


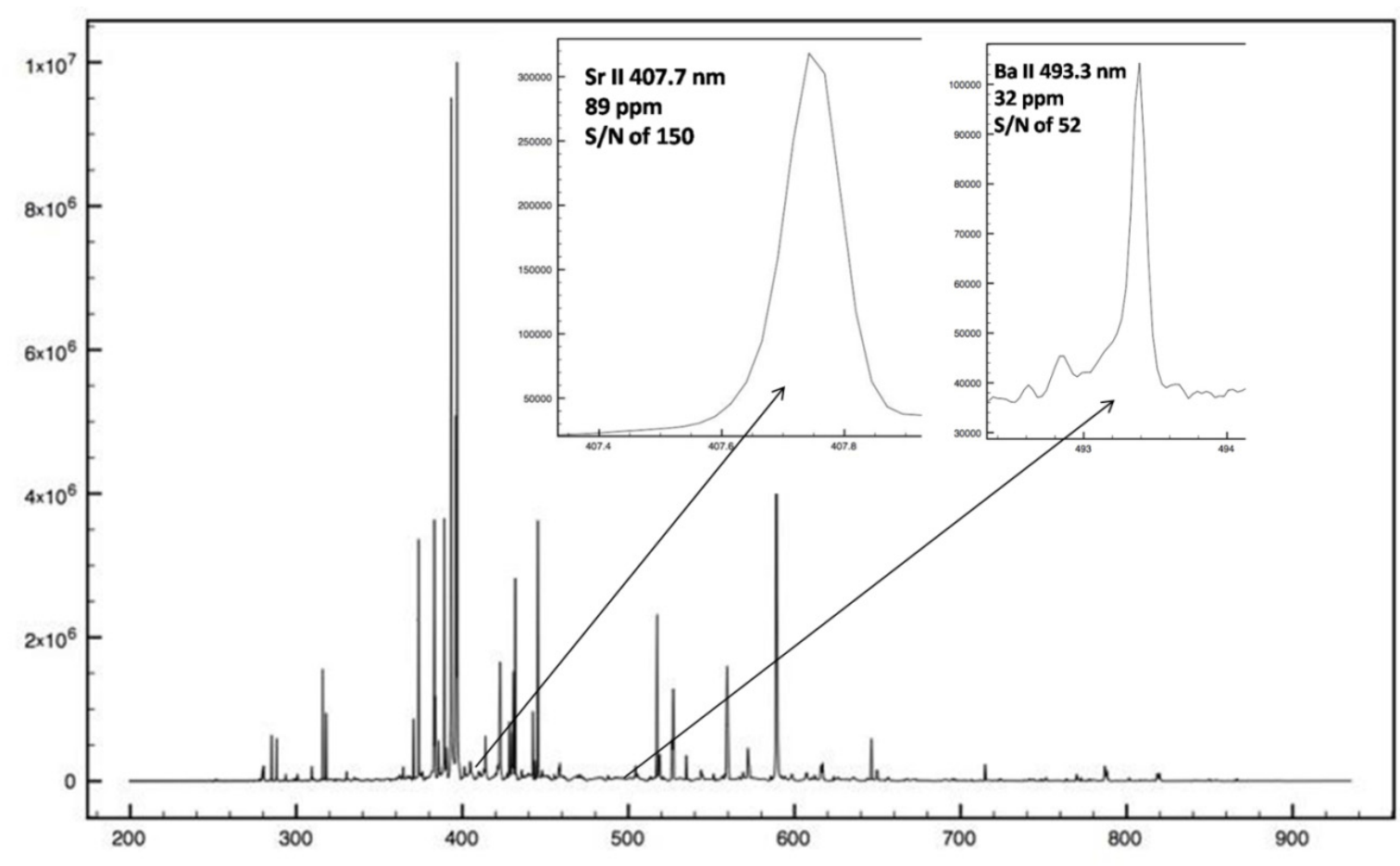

Figure 23. LIBS spectrum for CRM 1831float glass

The first condition for the elemental ratio was to provide correct association with the positive controls, CRM 1831 and the two duplicate samples from within the set. The sample CRM 1831 was analyzed repeatedly throughout the analysis, the beginning, middle (at minimum once or every two hours) and end of the experimental procedure. Two randomly chosen duplicate float glass samples were analyzed at random time intervals. The duplicate samples and the CRM 1831 standard were required to be found indistinguishable from itself at all analysis times. Requiring duplicate samples to be indistinguishable excludes ratios that would result in a Type I error (false exclusion). The elemental ratios were constructed using all possible pairs $[\mathrm{N}(\mathrm{N}-1) / 2, \mathrm{~N}=$ number of samples]. 
The second condition when choosing an elemental ratio was the percent discrimination it provided within the float glass samples. Ten (10) discriminating elemental ratios were used in each sample set. The elemental ratios vary between sample sets to provide the highest possible discrimination. 


\subsection{Statistical Analysis}

Pairwise comparisons were made using analysis of variance (ANOVA) and the General Linear Model (GLM) in Systat 11 (San Jose, Ca) with Tukey's honestly significant different test (HSD). The indistinguishable pairs produced by pairwise comparison analysis were then tested with a two sample t-test assuming unequal variances at a $95 \%$ confidence interval (Microsoft Excel, Redmond, WA). The samples found indistinguishable after the t-test were said to be indistinguishable, defined as having similar elemental profiles.

\subsubsection{Discrimination Results LIBS: Cardinal Glass Industries}

The Cardinal plant, sample set 1, had 49 samples producing 1176 possible pairs. The top 3 discriminating ratios were Al I 396.15/Ca I 534.95, K I 766.49/Na I 808.32 and K I $766.49 / \mathrm{Ca}$ I 616.22 discriminating $78.6 \%, 76.9 \%$ and $76.1 \%$ of the total possible pairs, respectively, while correctly associating duplicate samples and CRM 1831. All 10 discriminating ratios and their corresponding discrimination power are listed in Table 4 .

For method validation, CRM 1831 was analyzed 6 times throughout the experimental procedure and all 6 times all the replicates were found to be indistinguishable when compared to the other 5 runs with the statistical analysis previously described. Two samples were chosen to be analyzed a second time in the experimental procedure. The randomly chosen duplicate samples were, $01 / 14 / 98 \mathrm{~b}$ and 06/01/01b, and were used to assess for type I errors. Two sample pairs (01/14/98 \& 01/14/98 b, 06/01/01 \& 06/01/01 b) were found to be indistinguishable when compared with statistical analysis described above with the 10 discriminating elemental ratios. 
Table 6. LIBS Discriminating Ratios and the Percent Discrimination for Sample Set 1, Cardinal Glass Industries

\begin{tabular}{ccc}
\hline Cardinal & $\begin{array}{c}\% \\
\text { Ratios }\end{array}$ & $\begin{array}{c}\text { Pairs } \\
\text { discriminated }\end{array}$ \\
\hline Al I 396.15/Ca I 534.95 & 78.6 & 924 \\
K I 766.49/Na I 818.32 & 76.9 & 904 \\
K I 766.49/Ca I 616.22 & 76.1 & 895 \\
K I 766.49/Fe I 438.84 & 69.7 & 820 \\
Sr II 407.77/Al I 396.15 & 67.8 & 798 \\
Al I 394.40/Ba II 493.41 & 67.0 & 788 \\
Al I 396.15/Na I 818.32 & 65.7 & 773 \\
Fe I 371.64/A1 I 394.40 & 61.4 & 722 \\
Al I 394.40/Mg II 279.80 & 56.3 & 662 \\
Sr I 460.73/Ca I 616.22 & 55.8 & 656 \\
\hline
\end{tabular}

Pairwise comparison discriminated 1119 pairs out of the 1176 possible pairs yielding a discrimination of $95.2 \%$. Figure 24 shows the indistinguishable pairs by date. The $\mathrm{x}$-axis and the $\mathrm{y}$-axis are both labeled with the corresponding manufacturing dates and the dates range over the entire time of manufacture. Correlating a date on the y-axis with a date on the $\mathrm{x}$-axis with provide the two dates of the pairs found to be indistinguishable. The two dates will then provide the time separation in manufacture. It is observed that the most similar elemental composition is within approximately 2 months of manufacture. 


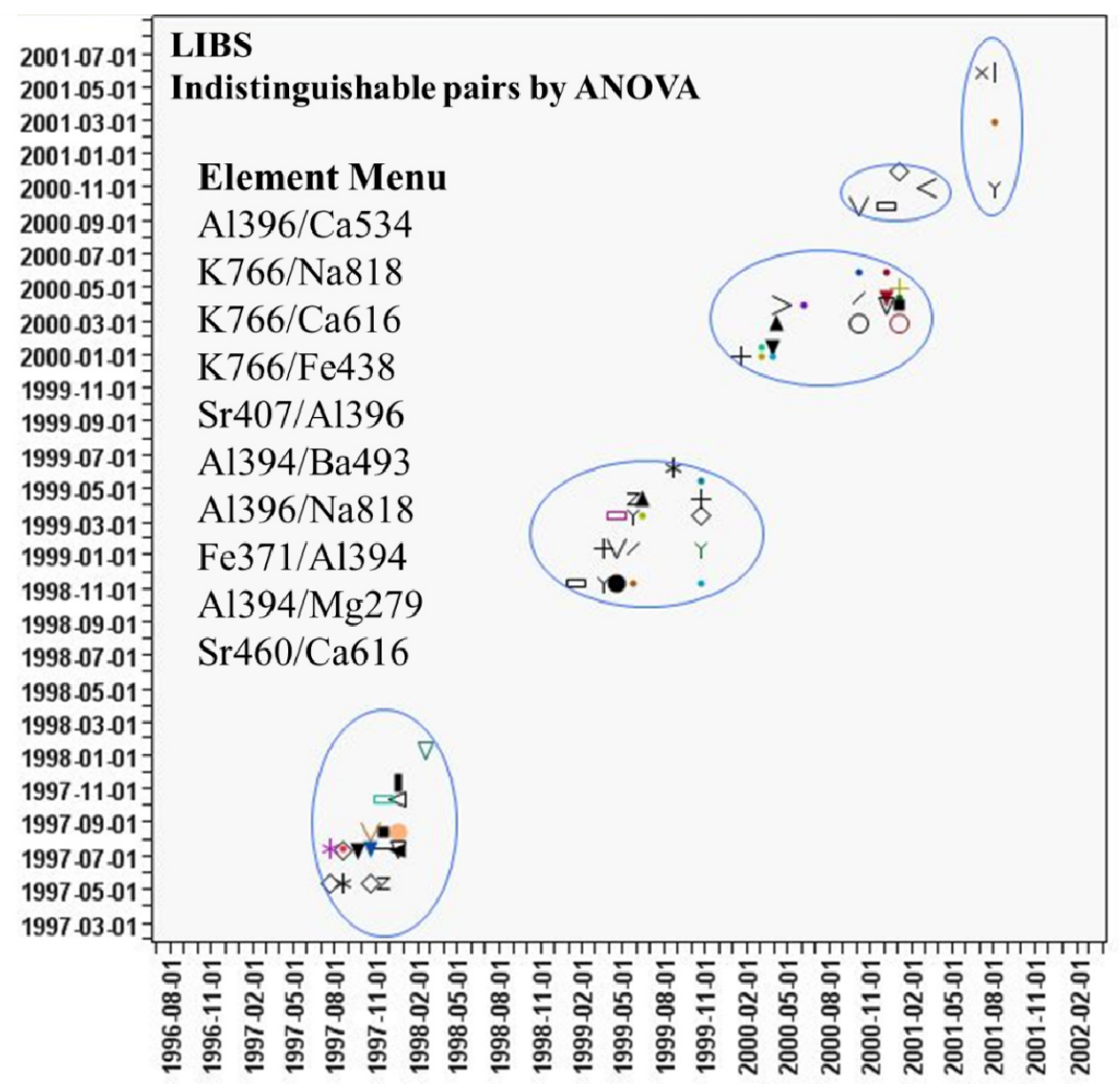

Figure 24. The indistinguishable pairs by ANOVA pairwise comparison using LIBS for sample set 1,

\section{Cardinal Glass Industries}

Fifty (50) of the 57 indistinguishable pairs were discriminated by the t-test,

producing a final discrimination of $99.4 \%$. Of the 1176 combinations, LIBS produced no type I error and 7 remaining indistinguishable pairs. Table 5 lists the indistinguishable pairs. The 7 indistinguishable pairs ranged from 1 month $(11 / 15 / 97 \& 12 / 15 / 97,03 / 15 / 99$ \& 04/14/99) apart to 15 months apart $(03 / 15 / 99 \& 06 / 01 / 01)$ in production time, with an average manufacture time separation of $\sim 4$ months. 
Table 7. Indistinguishable pairs by LIBS for Sample Set 1, Cardinal Glass Industries

\begin{tabular}{c}
\hline Cardinal Glass Industries \\
\hline $01 / 01 / 00 \&$ 03/27/00 \\
$04 / 01 / 01 \& 12 / 01 / 00$ \\
$03 / 01 / 01 \& 08 / 01 / 01$ \\
$06 / 01 / 01 \& 03 / 15 / 99$ \\
$08 / 17 / 97 \& 12 / 15 / 97$ \\
$11 / 15 / 97 \& 12 / 15 / 97$ \\
$03 / 15 / 99 \& 04 / 14 / 99$ \\
\hline
\end{tabular}

Interpretation of indistinguishable automotive float glass pairs by previous research is vague. Bridge et al. ${ }^{13,42}$ only reference the percent discrimination achieved with no details on actual sample dates and sources, i.e., make, model or year of car. Naes et al. and Schenk et al. ${ }^{3,27}$ are clear with float glass sample descriptions and suggest indistinguishable pairs of float glass are a result of the glasses originating from the same source, i.e., both inside and outside windshield glass, on the basis of the glasses sharing a similar manufacturing date. However, as a result of this information not being readily available, the dates of float glass manufacture to vehicle installation are unknown. The current research demonstrates that it is possible for glass to have similar reoccurring elemental profiles over a time period ranging from 1-15 months, with the most similarities occurring within approximately 2 months of manufacturing date.

\subsubsection{Discrimination Results LIBS: Pilkington North America, Inc.}

The Fe transitioned from high to low concentration from March 14, 2010 to March 19, 2010. The elemental compositions of ingredients will vary as a result of the desired end 
product. Iron is often varied in the manufacturing process, making it a good discriminating element. Iron is most often used to change the thermal emissivity of glass. Glass with low thermal emissivity values will not reflect thermal radiation, thereby increasing the transmittance.

Figure 25 demonstrates that LIBS is a sensitive analytical technique and is able to detect the differences in Fe concentration within a few days of production.

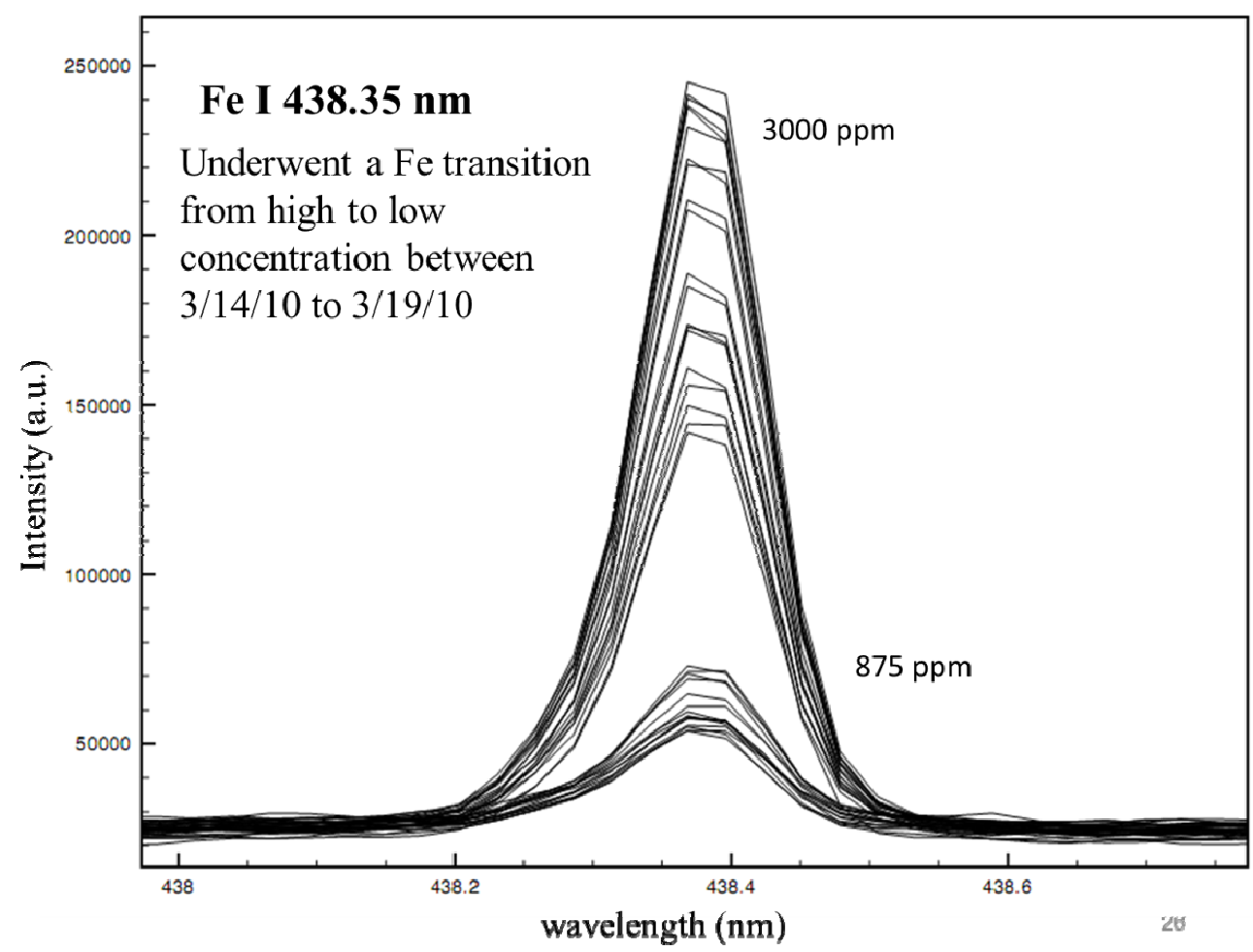

Figure 25. LIBS signal illustrating the high to low Fe transition at Pilkington North America Inc.

As a result of LIBS still being an invalidated technique in the forensic laboratory for the forensic analysis of glass, the LIBS results are compared to the well-established technique LA-ICP-MS for validation. The following chapter will cover LA-ICP-MS for the discrimination and association of float glass from a single manufacturing plant. The 
data for LA-ICP-MS is being compared here to demonstrate that LIBS is producing accurate data for the forensic analysis of glass.

Figure 26, seen below, illustrates that LIBS has a high correlation with LA-ICPMS. The independent axis reports the concentrations of Fe produced by LA-ICP-MS during the transition period. The dependent axis is the background subtracted LIBS intensities. The Pearson's product-moment correlation coefficient produced a $\mathrm{R}^{2}$ value of 0.992. The high value signifies that there is a high correlation produced between the relationship of the well-established LA-ICP-MS and LIBS, for the forensic analysis of glass.

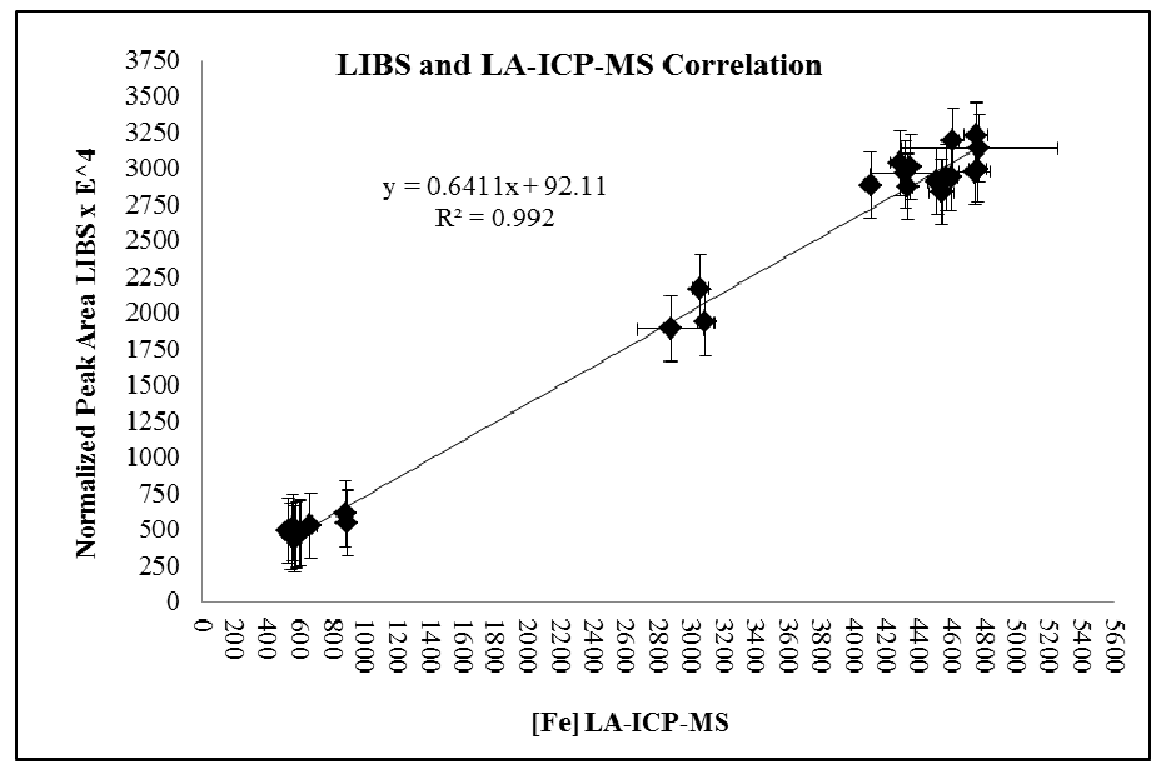

Figure 26. Correlation between LIBS and LA-ICP-MS for the Fe transition at Pilkington North America Inc.

Illustrated in Figure 27 is the correlation of LIBS and LA-ICP-MS for the elemental profiling of Fe for all 21 glass samples. In order to represent the data of LA-ICP-MS and LIBS on the same set of axes, the LIBS signal was multiplied by 100 and the LA-ICPMS concentrations were divided by a factor of 100 . 
Also observed in Figure 27, LIBS produces the same trend for signal intensities as does LA-ICP-MS for concentration of iron. The data represented illustrates the capability for LIBS to produce similar results as LA-ICP-MS data. Many forensic laboratories cannot not afford to acquire LA-ICP-MS instrumentation, in contrast, being less expensive, LIBS is proving to be an alternative elemental analysis technique.

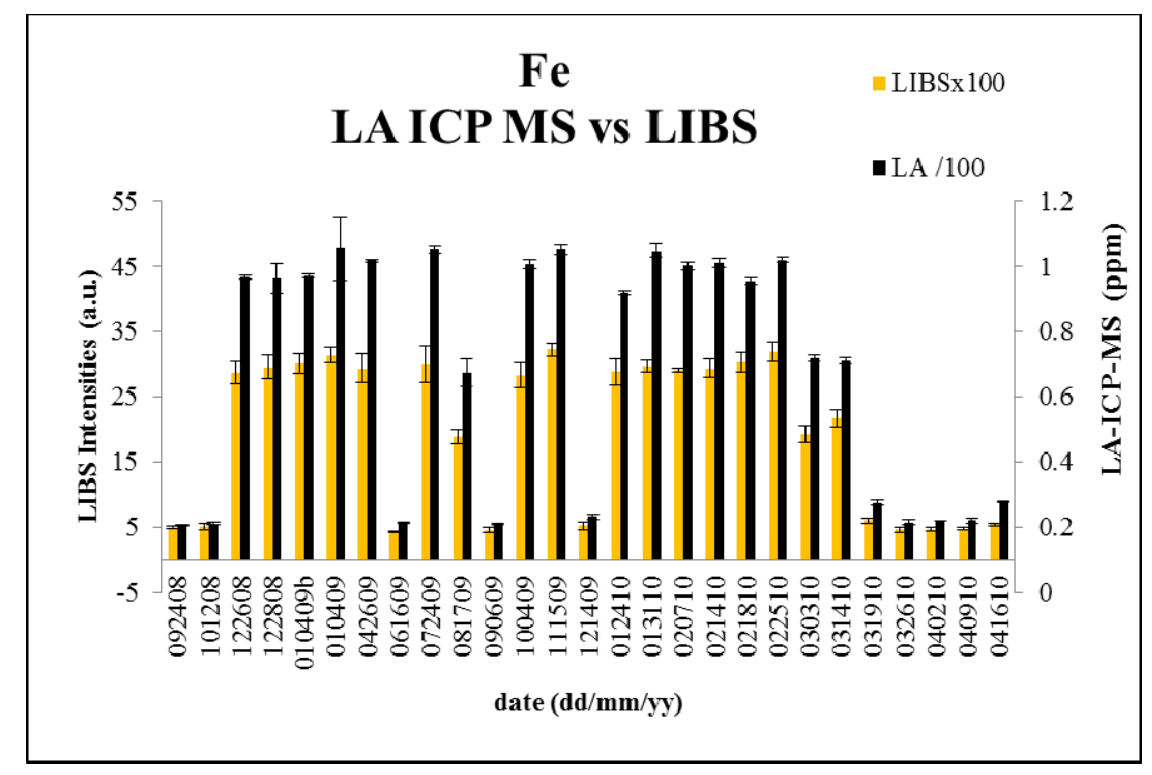

Figure 27. The elemental profile of Fe for sample set 2 by LIBS and LA-ICP-MS

The Pilkington plant, sample set 2, had 27 samples producing 351 possible pairs. The top 3 discriminating ratios were $\mathrm{Fe}$ I 438.84/Ti II 336.12, Fe I 438.84/Mg 517and Ca II $645.66 / \mathrm{Ti}$ I 365.35 discriminating $75.4 \%, 72.2 \%$ and $68.2 \%$ of the total possible pairs, respectively, while correctly associating duplicate samples and CRM 1831. Table 6 lists all 10 discriminating ratios and their corresponding discrimination power. 
Table 8. LIBS Discriminating Ratios and the Percent Discrimination for Sample Set 2, Pilkington North America, Inc.

\begin{tabular}{ccc}
\hline Pilkington & $\%$ & Pairs \\
Ratios & discrimination & discriminated \\
\hline Fe I 438.84/Ti II 336.12 & 75.4 & 265 \\
Fe I 438.84/Mg 517 & 72.2 & 253 \\
Ca II 645.66/Ti I 365.35 & 68.2 & 239 \\
Sr 460/Ti I 365.35 & 68.0 & 239 \\
Al I 394.40/Fe I 438.84 & 62.3 & 219 \\
Fe I 438.84/Na I 330.13 & 61.1 & 215 \\
Sr II 407.77/Fe I 438.84 & 60.3 & 212 \\
Sr I 460.73/K I 766.49 & 50.2 & 176 \\
Al I 396.15/Mg 280 & 44.6 & 157 \\
K I 766.49/Na I 330.13 & 44.1 & 155 \\
\hline
\end{tabular}

The CRM 1831 was analyzed 3 times throughout the experimental procedure and no significance differences were determined. Two duplicate samples, 01/04/09b and 10/12/08b, were analyzed an extra time during the analysis. The pairs $(01 / 04 / 09 b \&$ 01/04/09, 10/12/08b \& 10/12/08) were found to be indistinguishable when compared with statistical analysis using the 10 discriminating elemental ratios.

Pairwise comparison produced 111 indistinguishable pairs (240 distinguished), resulting in a $68.4 \%$ discrimination. The t-test further discriminated another 107 out of the 111 indistinguishable pairs, producing a discrimination of $98.8 \%$. The remaining 4 indistinguishable pairs, see Table 7, range from only 2 weeks to 2 months apart. 
Table 9. Indistinguishable pairs by LIBS for Sample Set 2, Pilkington North America, Inc.

Pilkington North America, Inc.

$03 / 03 / 10 \& 03 / 14 / 10$

03/03/10 \&02/07/10

$10 / 12 / 08 \& 12 / 28 / 08$

$01 / 31 / 10 \& 02 / 07 / 10$

Overall, LIBS produced an average precision throughout the experiment in sample set one of $\sim 6 \%$ RSD for all 490 combinations (10 ratios, 49 samples). The average precision in sample set 2 (270 combinations) was $\sim 5 \%$ RSD throughout the entire experiment. The figures of merit for LIBS on CRM 1831 are list in Table 8, demonstrating the precision and accuracy of LIBS. The precision of LIBS can be as good as $1.5 \%$ RSD for K I $766.49 \mathrm{~nm}$ emission line and a bias of $1.0 \%$ for the Ba II $493.41 \mathrm{~nm}$ emission line.

Table 10. LIBS Figures of Merit for CRM 1831

\begin{tabular}{ccccccc}
\hline CIST 1831 & $\begin{array}{c}\text { Conc. } \\
(\mathrm{ppm})\end{array}$ & SNR & $\begin{array}{c}\text { Precision } \\
\text { \% RSD }\end{array}$ & $\begin{array}{c}\% \\
\text { Bias }\end{array}$ & $\begin{array}{c}\text { LOD } \\
(\mathrm{ppm})\end{array}$ & $\begin{array}{c}\text { LOQ } \\
(\mathrm{ppm})\end{array}$ \\
\hline Al I 394.4 & 6381 & 277 & 2.5 & 2.0 & 70.7 & 235.6 \\
Ba II 493.41 & 32 & 63 & 4.6 & 1.0 & 1.1 & 3.6 \\
Sr I 460.73 & 89 & 285 & 4.0 & 3.6 & 0.9 & 2.9 \\
Ca I 534.95 & 58604 & 1851 & 3.4 & 7.6 & 68.0 & 226.9 \\
Fe I 438.44 & 802 & 40 & 3.1 & 16.0 & 21.0 & 70.1 \\
K I 766.49 & 2738 & 1133 & 1.5 & 2.6 & 3.9 & 13.1 \\
Mg II 279.80 & 21166 & 367 & 2.4 & 12.3 & 82.1 & 274.3 \\
Na II 330.13 & 98825 & 1052 & 4.1 & -- & 190.7 & 635.7 \\
\hline
\end{tabular}




\subsection{Round Robin Glass Studies}

In efforts to standardize methods for the forensic analysis of glass, a grant by the National Institute of Justice was awarded to my mentor, Dr. Almirall. I was privileged to be able to participate in this working group as it consisted of both national and international analytical and forensic scientists. The working group provided me the opportunity to interact with working scientists.

The group was named the Elemental Analysis Working Group (EAWG) and to date I have been involved in five meetings. The objective of EAWG was to conduct elemental analysis of glass with different analytical techniques to improve the analytical method used for characterization and comparison, to evaluate the different statistical methods to determine match criteria, interpretation of these results and finally the reporting of these results. The group focused on LA-ICP-MS, ICP-MS, $\mu$ XRF and LIBS. Only the LIBS portion of this working group will be compared, as my role was to represent FIU for the forensic analysis of glass using LIBS for four meetings that took place in August 2008, December 2009, August 2010 and December 2010, starting with round robin two.

It is important to note, that the first two inter-laboratory studies, FIU was the only LIBS laboratory, where in the fourth inter-laboratory study the LIBS participants increased to 5 laboratories, demonstrating the growth for LIBS as an analytical technique for the forensic analysis of glass.

Round robin two was an inter-laboratory study conducted on float glass standards NIST 1831, FGS 1 and FGS 2 to study the measurement variation and inter-laboratory 
variation ${ }^{166}$. FGS 1 and FGS 2 are float glass standards produced by SCHOTT, Germany. These standards resemble soda-lime glass composition; however the elemental concentrations are different by a factor of 5 . In addition to the float glass standards, an additional set of glass fragments were also analyzed. These second set of glass fragments were submitted for comparison to evaluate analytical results produced inter-laboratory.

The elemental analysis of glass results were separated into two groups. As a result of FIU being the only LIBS participant, LIBS was grouped with 7 XRF participants. In summary all participants in of this inter-laboratory study received instructions for analysis and the following glass samples with a given forensic scenario:

1) NIST 1831: Full thickness fragment

2) NIST 1831: small fragments

3) FGS 1: small glass fragments

4) FGS 2: small glass fragments

5) Item 1, known glass sample (K1): three glass fragments $(2-7 \mathrm{~mm})$ collected from broken window of victim's house.

6) Item 2, question sample (Q1): three glass fragments (1-4 mm) recovered from the suspect's shirt.

7) Item 3, question sample (Q2): three glass fragments (1-4 mm) collected from suspect's pants.

A likely crime scenario was provided and each participant was to associate or distinguish the known sample $(\mathrm{K})$ to the questioned sample $(\mathrm{Q})$. After results were submitted, it became known that $\mathrm{K} 1$ and Q1 samples were of the same origin, the two samples 
originated from the same glass pane. Sample Q2 had originated from a separate pane of glass produced from the same plant, manufactured $\sim 3$ years previously. All participants in group 1 and 2 correctly associated samples K1 with Q1 and discriminated K1 with $\mathrm{Q} 2^{166}$. The match criteria used for the XRF labs was spectra overlay with \pm 2 or \pm 3 standard deviations. The match criteria used for LIBS was ANOVA with student's t-test at a $95 \%$ confidence interval using elemental ratios $\mathrm{Al} / \mathrm{Sr}, \mathrm{Fe} / \mathrm{Sr}, \mathrm{Ca} / \mathrm{K}, \mathrm{Al} / \mathrm{Ca}, \mathrm{Al} / \mathrm{Na}$, $\mathrm{Ba} / \mathrm{Sr}$ and $\mathrm{Ca} / \mathrm{Sr}$.

The accuracy and precision of the standard reference materials were also evaluated. However, as a result of FIU being the only LIBS laboratory, the interlaboratory accuracy/precision studies could not be compared. Different match criteria, as chosen independently by the analyst, all provided the correct results. In conclusion, this study indicated that LIBS is able to provide similar results as LA-ICP-MS and XRF.

Round robin three was composed of glass samples NIST 1831 and two glass sets. Set one was from the same manufacturing plant and was selected to study the capabilities of the different techniques to discriminate between different time intervals of glass production. This set consisted of 2 known samples and 3 questioned samples. The second set was to evaluate the elemental variation within container glass. As usual, all sample sets are distributed without the known associations provided. After results are submitted the association data is provided. This inter-laboratory study consisted of 1 ICPMS, 5 LA-ICP-MS, 4 XRF and 2 LIBS laboratories. Unfortunately, as a result of the two LIBS method being very different, there could be no comparisons made between the two LIBS laboratories. However, a comparison of the analytical results of LIBS to both the established techniques of LA-ICP-MS and XRF are compared. 
All samples were previously analyzed by LA-ICP -MS and concentrations documented in the glass database at FIU. After analysis the results were provided that samples K2 versus Q1, K1 versus Q2 and K1 versus Q3, all have fairly distinct element profiles. Samples with similar elemental profiles were K2 versus Q1, K1 versus Q2, and K1 versus Q3. The dates of manufacture for the $\mathrm{K}$ and $\mathrm{Q}$ samples are listed in Table 9.

Table 11. Description of Sample Set One for Round Robin Three

\begin{tabular}{|c|c|}
\hline Database I.D. & Sample \\
\hline 8/17/2001 & K1 \\
\hline 8/31/2001 & Q1 \\
\hline 4/15/1998 & K2 \\
\hline 5/17/1998 & Q2 \\
\hline $7 / 17 / 1998$ & Q3 \\
\hline
\end{tabular}

Again a scenario was developed to mimic a potential crime scene. The LIBS results obtained are listed in Table 10 with the corresponding discriminating ratios. An " $x$ " in the box signifies a significant difference between the elemental composition for that specified ratio.

Table 12. LIBS Discriminating Ratios and Results for Round Robin Three

\begin{tabular}{|c|c|c|c|c|c|c|c|c|}
\hline \multicolumn{9}{|l|}{$\mathrm{x}$ : different } \\
\hline $\begin{array}{c}\text { Summary } \\
\text { systat }\end{array}$ & $\begin{array}{c}\text { Na } 818 \\
\text { K } 766\end{array}$ & $\begin{array}{l}\text { K766 } \\
\text { Ca } 643\end{array}$ & $\begin{array}{c}\text { Fe438 } \\
\text { K766 }\end{array}$ & $\begin{array}{l}\text { Ca534 } \\
\text { K766 }\end{array}$ & $\begin{array}{l}\text { Al394 } \\
\text { Sr460 }\end{array}$ & $\begin{array}{l}\text { Al394 } \\
\text { Na330 }\end{array}$ & $\begin{array}{l}\text { Sr460 } \\
\text { K766 }\end{array}$ & $\begin{array}{l}\text { Fe } 438 \\
\text { Al } 396\end{array}$ \\
\hline \multicolumn{9}{|l|}{ K2 vs } \\
\hline Q1 & $x$ & $x$ & $\mathrm{x}$ & $x$ & $x$ & $x$ & $x$ & $x$ \\
\hline Q2 & $x$ & & $x$ & $x$ & $x$ & $x$ & $x$ & $x$ \\
\hline Q3 & $x$ & & $\mathrm{x}$ & $x$ & & & $x$ & \\
\hline \multicolumn{9}{|l|}{$\mathrm{x}$ : different } \\
\hline $\begin{array}{c}\text { Summary } \\
\text { systat }\end{array}$ & $\begin{array}{c}\text { Na } 818 \\
\text { K } 766\end{array}$ & $\begin{array}{l}\text { K766 } \\
\text { Ca } 643\end{array}$ & $\begin{array}{c}\text { Fe438 } \\
\text { K766 }\end{array}$ & $\begin{array}{l}\text { Ca534 } \\
\text { K766 }\end{array}$ & $\begin{array}{l}\text { Al394 } \\
\text { Sr460 }\end{array}$ & $\begin{array}{l}\text { Al394 } \\
\text { Na330 }\end{array}$ & $\begin{array}{l}\text { Sr460 } \\
\text { K766 }\end{array}$ & $\begin{array}{l}\text { Fe } 438 \\
\text { Al } 396\end{array}$ \\
\hline \multicolumn{9}{|l|}{ K1 vs } \\
\hline \multicolumn{9}{|l|}{ Q1 } \\
\hline Q2 & $x$ & $x$ & $\mathrm{x}$ & $x$ & $x$ & & $x$ & \\
\hline Q3 & $x$ & $x$ & $\mathrm{x}$ & $x$ & $x$ & $x$ & $x$ & $x$ \\
\hline
\end{tabular}


In the above table, $\mathrm{K} 2$ is found significantly different than all the questioned samples. K1 is found significantly different than Q2 and Q3, while no significant differences are produced between the elemental composition of $\mathrm{K} 1$ and Q1.

The inter-laboratory comparisons are best illustrated in the following Table $11^{167}$ and Table $12^{167}$ where $\mathrm{K} 1$ and $\mathrm{K} 2$ are compared to the questioned samples. H LIBS signifies my results.

Table 13. Final Round Robin 3 Report, Comparison of K1 to Questioned Samples ${ }^{167}$

\begin{tabular}{|c|c|c|c|c|}
\hline Lab ID & K1 vs Q1 & K1 vs Q2 & K1 vs Q3 & Match criteria \\
\hline A XRF & IN & DS & DS & Spectra overlap \\
\hline B XRF & IN & DS & DS & $\begin{array}{l}\text { Spectra overlap, } \pm 3 \text { s of ratio intensities } \mathrm{Ca} / \mathrm{Mg} \text {, } \\
\mathrm{Ca} / \mathrm{Ti} \mathrm{Ca} / \mathrm{Fe}, \mathrm{Sr} / \mathrm{Zr}, \mathrm{Fe} / \mathrm{Zr}, \mathrm{Ca} / \mathrm{K}, \mathrm{Fe} / \mathrm{Sr}, \mathrm{Fe} / \mathrm{Mn}\end{array}$ \\
\hline C XRF & IN & DS & DS & $\begin{array}{l}\text { Spectra overlap, } \pm 3 \text { s of ratio intensities } \\
\text { Excluded by } \mathrm{Ca} / \mathrm{Ti}, \mathrm{Ca} / \mathrm{K} . \mathrm{Ca} / \mathrm{Mn}\end{array}$ \\
\hline E XRF & IN & DS & DS & Spectra overlap, \pm 3 s of ratio intensities \\
\hline F XRF & IN & DS & DS & $\begin{array}{c} \pm 3 \text { s of ratio intensities } \mathrm{Ca} / \mathrm{Fe}, \mathrm{Sr} / \mathrm{Zr}, \mathrm{Ca} / \mathrm{K} \\
\mathrm{Fe} / \mathrm{Mn}, \mathrm{Ca} / \mathrm{Mn}, \mathrm{Fe} / \mathrm{Ti}, \mathrm{Ca} / \mathrm{Ti}\end{array}$ \\
\hline H LIBS & IN & DS & DS & Elemental ratios, ANOVA + Tukey $\mathrm{p}=0.05$ \\
\hline I LIBS & IN & $\mathrm{IN}^{* 4}$ & DS & PLS algorithm \\
\hline A ICP & $\mathrm{IN} * 2$ & DS & DS & $\pm 2 \mathrm{~s}$ \\
\hline B ICP & DS & DS & DS & $\pm 2 \mathrm{~s}$ and $\pm 3 \mathrm{~s}$ \\
\hline C ICP & DS & DS & DS & modified $\pm 4 \mathrm{~s}$ \\
\hline D ICP & $\mathrm{DS}^{* 1}$ & DS & DS & $\begin{array}{c}\mathrm{t} \text { test }\left(\text { Bonferroni correction), } \mathrm{p}=0.05,{ }^{*} \text { ANOVA }\right. \\
+ \text { Tukey } \mathrm{p}=0.05\end{array}$ \\
\hline E ICP & IN $*^{2}$ & DS & DS & $t$ test $p=0.05$ and ANOVA $(p=0.05)$ \\
\hline F ICP & IN $*^{2}$ & DS & $\mathrm{IC}^{* 3}$ & Range overlap and $\pm 3 \mathrm{~s}$ \\
\hline H ICP & DS & DS & DS & modified $\pm 4 \mathrm{~s}$ \\
\hline
\end{tabular}


In the above Table 11, the following notations and definitions signify: IN: samples are indistinguishable, DS: samples are distinguishable, IC: inconclusive, *: sample Q3 suffer problems during digestion, therefore the precision does not allow a fair comparison, $* 2$ : participant reported indistinguishable, but t-test of raw data shows significant differences on some elements ${ }^{167}$.

The K1 versus Q1 samples are manufactured approximately two weeks apart, therefore it is assumed that their elemental composition would be similar as a result of previous conducted studies. It is observed that only 4 LA-ICP-MS laboratories and I, using LIBS, found these samples to be distinguished. This sample pair was distinguished by the $\mathrm{Na} / \mathrm{K}$ ratio, where most ICP labs found $\mathrm{Ba}$ to be one of the most discriminating elements.

The K1 versus Q2 and K1 and Q3 samples were found to be distinguishable by most all laboratories. These results were expected, as a result of previously conducted research, that these two sample pairs would have a distinct elemental profiles as a result of their manufacturing time being a difference of approximately 3 years.

Table 14. Final Round Robin 3 Report, Comparison of K2 to Questioned Samples ${ }^{167}$

\begin{tabular}{|c|c|c|c|c|}
\hline Lab ID & K2 vs Q1 & K2 vs Q2 & K2 vs Q3 & Match criteria \\
\hline A XRF & DS & DS & IN & Spectra overlap \\
\hline B XRF & DS & DS & IN & $\begin{array}{l}\text { Spectra overlap, } \pm 3 \text { s of ratio intensities } \mathrm{Ca} / \mathrm{Mg} \text {, } \\
\mathrm{Ca} / \mathrm{Ti} \mathrm{Ca} / \mathrm{Fe}, \mathrm{Sr} / \mathrm{Zr}, \mathrm{Fe} / \mathrm{Zr}, \mathrm{Ca} / \mathrm{K}, \mathrm{Fe} / \mathrm{Sr}, \mathrm{Fe} / \mathrm{Mn}\end{array}$ \\
\hline C XRF & DS & IN & IN & $\begin{array}{l}\text { Spectra overlap, } \pm 3 \text { s of ratio intensities } \\
\text { Excluded by } \mathrm{Ca} / \mathrm{Ti}, \mathrm{Ca} / \mathrm{K} . \mathrm{Ca} / \mathrm{Mn}\end{array}$ \\
\hline E XRF & DS & IN & IN & Spectra overlap, \pm 3 s of ratio intensities \\
\hline F XRF & DS & DS & IN & $\begin{array}{l} \pm 3 \text { s of ratio intensities } \mathrm{Ca} / \mathrm{Fe}, \mathrm{Sr} / \mathrm{Zr}, \mathrm{Ca} / \mathrm{K} \\
\mathrm{Fe} / \mathrm{Mn}, \mathrm{Ca} / \mathrm{Mn}, \mathrm{Fe} / \mathrm{Ti}, \mathrm{Ca} / \mathrm{Ti}\end{array}$ \\
\hline H LIBS & DS & DS & DS & Elemental ratios, ANOVA + Tukey $p=0.05$ \\
\hline
\end{tabular}




\begin{tabular}{|c|c|c|c|c|}
\hline I LIBS & DS & DS & IN & PLS algorithm \\
\hline A ICP & DS & DS & $\mathrm{IN}^{* 2}$ & $\begin{array}{c} \pm 2 \text { s (for } 10 \text { elements menu, if number of } \\
\text { overlaps } 9 \text { or } 10 \text { then match if }<9 \text { then non- } \\
\text { match) }\end{array}$ \\
\hline B ICP & DS & DS & DS & $\pm 2 \mathrm{~s}$ and $\pm 3 \mathrm{~s}$ \\
\hline C ICP & DS & DS & DS & modified $\pm 4 \mathrm{~s}$ \\
\hline D ICP & DS & DS & DS & $\begin{array}{c}\mathrm{t} \text { test (Bonferroni correction, } \mathrm{p}=0.05 \text { ), ANOVA } \\
+ \text { Tukey, } \mathrm{p}=0.05\end{array}$ \\
\hline E ICP & DS & DS & IN $^{* 2}$ & $\mathrm{t}$ test at $95 \%$ and ANOVA $(95 \%)$ \\
\hline F ICP & DS & DS & $\mathrm{IC}$ * & Range overlap and $\pm 3 \mathrm{~s}$ \\
\hline H ICP & DS & DS & DS & modified $\pm 4 \mathrm{~s}$ \\
\hline
\end{tabular}

In the above Table $12^{167}$, the following notations and definitions signify: IN: samples are indistinguishable, DS: samples are distinguishable, IC: inconclusive, ${ }^{*}$ : sample Q3 suffer problems during digestion, therefore the precision does not allow a fair comparison, *2: participant reported indistinguishable, but $t$-test of raw data shows significant differences on some elements ${ }^{167}$.

The K2 versus Q1 and K2 versus Q2 samples are manufactured approximately 3 years apart and are found distinguishable by most laboratories. The K2 versus Q2 samples are found indistinguishable by two XRF laboratories. Sample pair K2 and Q3 were manufactured 3 months apart. All XRF participants and the other LIBS participant found these samples to be indistinguishable. These results demonstrate that LIBS may provide more sensitivity than the well-established analytical method XRF and competes as an analytical method for the forensic analysis of glass against LA-ICP-MS when operated under optimized conditions. 
Sample set two in round robin two was a green Perrier water bottle that was broken and the glass fragments were analyzed, see Figure 28.

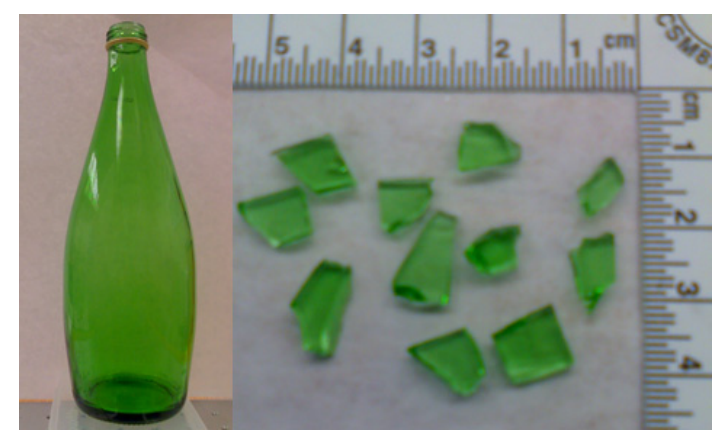

Figure 28. Sample Set 2, Perrier bottle, for round robin three

Five fragments from the bottle were sampled to study the homogeneity. Samples B1, B2 and B3 were all taken from the center of the bottle, B4 from the lower portion and B5 was taken from the bottle neck. The following Table 13 demonstrates the discriminating ratios used for analysis.

It has been documented that the elemental composition in container glass has greater heterogeneity than float glass ${ }^{41}$. The heterogeneity is often contributed the manufacturing process as a result of the molding parts providing contamination ${ }^{41}$. In previous research by Trejos et al. it was reported that the inherent variation within the elemental composition of the container is greater than the instrumental variation using LA-ICP-MS ${ }^{41}$. 
Table 15. Round Robin Three Perrier Bottle Analyses

\begin{tabular}{|c|c|c|c|c|c|c|c|}
\hline X=different & Ca534 & Al396 & Al394 & Al394 & Al394 & Na330 & Ca645 \\
\hline Summary systat & Sr460 & Ca534 & Sr460 & Ca534 & Fe438 & Si288 & Mg 279 \\
\hline & & & & & & & \\
\hline B1 & & & & & & & \\
\hline B2 & & & X & & X & & \\
\hline B3 & & & & & & & \\
\hline B4 & & & & & & & \\
\hline B5 & & & & & & & \\
\hline
\end{tabular}

To evaluate the variation between the different analytical techniques, all statistical analyses were done in the same manner using ANOVA, followed by a t-test at a $95 \%$ confidence interval. The LIBS data I produced found significant differences between pairs $\mathrm{B} 2$ and $\mathrm{B} 4$ with the elemental ratio $\mathrm{Al} / \mathrm{Sr}$ and between pair $\mathrm{B} 3$ and $\mathrm{B} 2$ with elemental ratio $\mathrm{Al} / \mathrm{Fe}$, see Table 13.

All participants found significant differences between the container set except for solution ICP-MS where the total mass samples is milligrams compared to LIBS consuming nanograms to micrograms. The results obtained here demonstrate that more samples need to be analyzed to produce an accurate elemental profile for container glass for all micro-sampling techniques.

The final round robin for which I participated in concentrated on statistical analysis from round robin 3 and round robin 4 to evaluate the effect on type I and type II errors ${ }^{168}$. The sample set of round robin 4 was also from a single manufacturing plant. This set consisted of 2 known glass fragments and 3 questioned samples. Round Robin 4 had 3 participants report results using LIBS.

All participants performed the following statistical tests for match criteria for data from round robin 3 and round robin 4: 1) range overlap, 2) t-test at 99\% confidence 
interval, 3) t-test at a 95\% confidence interval, 4) t-test with Bonferoni correction, 5) \pm 2 standard deviation, 6) \pm 3 standard deviation, 7) \pm 4 standard deviation and 8) Hotelings T.

Using the FIU glass database, sample pairs for round robin 4 with similar elemental composition were K2 versus Q1. Sample Q1 was manufactured on 02/18/2010 while all other samples, K1, K2, Q2 and Q3 all originated from the same date, 03/03/2010. The known fragments were provided to participants at full size, meaning there was original surface of both the float and non-float side, and the questioned samples were provided as irregular fragments $(0.5-1 \mathrm{~mm})$.

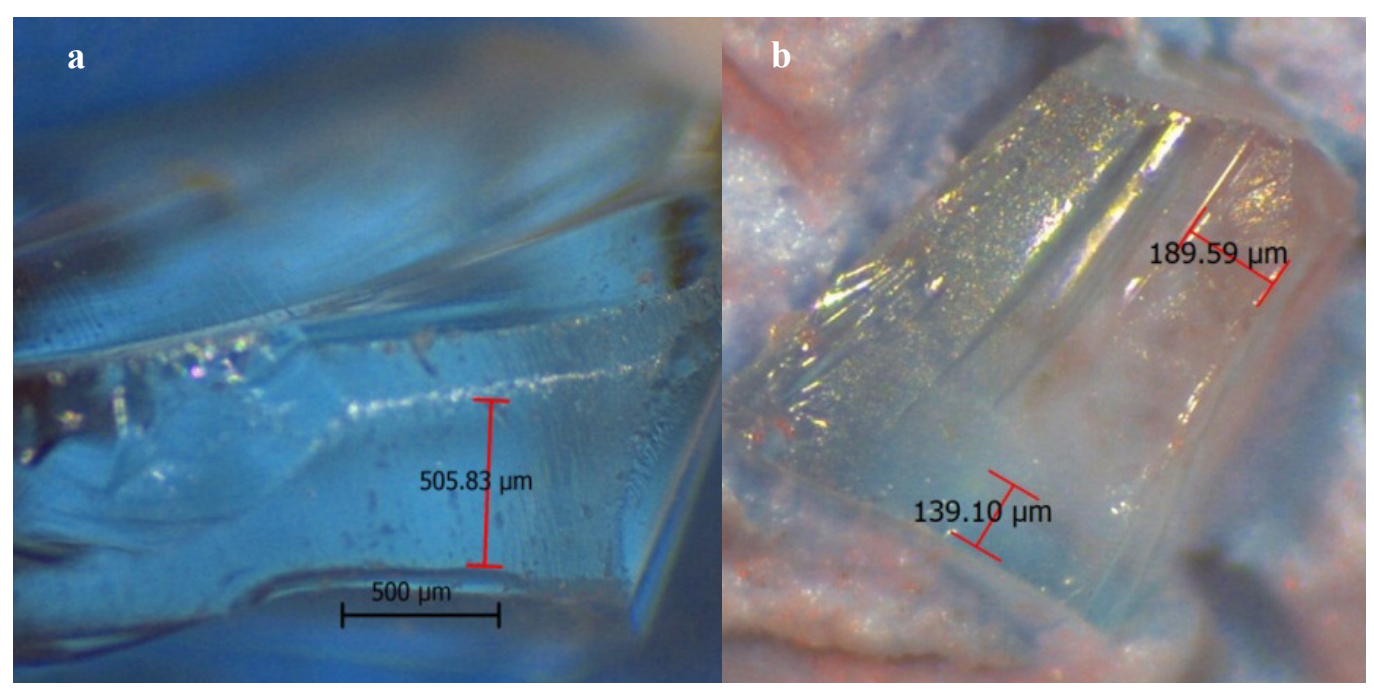

Figure 29. Glass fragments provided for round robin four

To provide reproducible results for LIBS analysis the glass fragments were mounted in a soft modeling compound, orientated so that a flat non-float surface can be ablated, see Figure 29. Figure 29a is sample K1, it is evident, that even as a full thickness fragment, it is still very small. Figure 29b is sample Q1. Care needed to be given to all samples, so that there would be space for 5 replicates with a laser spot size of $\sim 100 \mu \mathrm{m}$ for LIBS analysis. 
First, all participants provided results using their selected match criteria. All participants responded correctly and found Q1 to be distinguishable samples from K1 and K2. I was the only LIBS participant to correctly report that Q1 to be distinguished from samples $\mathrm{K} 1$ and $\mathrm{K} 2$. This is explained as the glass method I have developed has been optimized and validated with FIU LIBS instrumentation.

Unfortunately, the LIBS data varied greatly between laboratories. The lack of standardization inter-laboratory using LIBS, such as; instrument laser wavelength, temporal parameters and data treatment, produced a large variation in LIBS results. LIBS, still being a novel technique for the forensic analysis of glass, is not yet standardized within the LIBS community resulting in inter-laboratory inconsistency. More studies need to be conducted with LIBS before a match criteria can be suggested. Match criteria methods were focused on for ICP and XRF techniques as a result of having method standardization. It was determined that match criteria needs to be selected with care based on elemental technique used. Analytical instrumentation achieving precision of $\leq 2 \%$ RSD may be too sensitive and produce false exclusions for match criteria using the t-test. The false exclusion rate was significantly reduced by using a $3 \%$ RSD and a match criteria of \pm 4 standard deviations ${ }^{168}$. 


\subsection{Conclusions}

Laser induced breakdown spectroscopy was shown to be a powerful and sensitive technique for the forensic association and discrimination of float glass. The elemental concentrations of float glass varies during the manufacturing process. These variations provide discrimination of float glass samples within the same manufacturing plants from days to years apart. LIBS has the ability to detect the small variations within the elemental profile. Eighteen (18) emission lines corresponding to the elements $\mathrm{Sr}, \mathrm{K}, \mathrm{Fe}$, $\mathrm{Ca}, \mathrm{Al}, \mathrm{Ba}, \mathrm{Na}, \mathrm{Mg}$ and $\mathrm{Ti}$, were chosen because of their detection above the method detection limits and for presenting differences between the samples. The elemental ratios producing the most discrimination in the first sample set, Cardinal Glass Industries, were; Al I (396.15)/Ca I (534.95), K I (766.49)/Na I (808.32) and K I (766.49)/Ca I (616.22) discriminating $78.6 \%, 76.9 \%$ and $76.1 \%$, respectively, of the possible 1176 pairs. The elemental ratios producing the most discrimination in the second sample set, Pilkington North America, Inc., were; Fe I (438.84)/Ti II (336.12), Fe I (438.84)/Mg I (517.27) and $\mathrm{Ca}$ II (645.66)/Ti I (365.35) discriminating 75.4\%, 72.2\% and 68.2\% respectively, of the possible 351 pairs. When all the ratios are combined in a comparison, $99 \%$ of the possible pairs were discriminated from two different float glass sample sets manufactured from a day to years apart, using the optimized LIBS method. LIBS achieved good precision $(\sim 5 \%$ RSD) throughout both studies. These results support the hypothesis that the elemental composition of glass produced in a single plant over time varies more than the differences that can be detected using analytical LIBS. The robustness of this technique is demonstrated with the production of no type I errors. 
The present research demonstrated that similar elemental profiles are possible from 1-15 months apart. However, data supports the most similar elemental profile being within 1-3 months of manufacture.

The LODs achieved by LIBS are below the expected ranges encountered in the elemental concentration of float glass, making LIBS a fit technique for the forensic analysis of glass. LIBS demonstrates to compete with well-established methods of LAICP-MS and XRF for the forensic analysis of glass. However, if LIBS is to be the next analytical method found commonly in the forensic laboratory, standardization of instrumentation, methodology and match criteria need to be assessed. 


\section{Laser Ablation Inductively Coupled Mass Spectrometry for the Discrimination and Association of Float Glass from Single Manufacturing Plants}

Previous conducted research documents the discrimination between float glass ${ }^{14,169-173}$. Bridge et al. ${ }^{169}$ analyzed 27 float glass samples originating side window and side mirror glass collected from automobiles. These authors report a 74\% discrimination using LAICP-MS. However, Naes et al. demonstrate that $99 \%$ of the automotive samples from within the same car can be discriminated ranging in year from 1998 to 2004. The author's state that the $1 \%$ of indistinguishable pairs are explained by the pairs originating from the same vehicle and that this is a result of the float glass having been manufactured at the same plant at approximately the same time ${ }^{170}$. To date, however, no studies have reported the association and discrimination of float glass from a single plant in such short periods of time with LA-ICP-MS. The next section focuses on the capability for LA-ICPMS to discriminate float glass produced at the same plant at approximately the same time period.

\subsection{Glass Sample Set for LA-ICP-MS}

The sample set composed of 27 colorless float glass samples collected from September 2008 to April 2010. These samples were manufactured in Pilkington North America Inc. (Stockton, CA, USA). The refractive indices of these samples were not determined due to previous research demonstrating the narrow spread of data. Table 3 summarizes sample set two. This is the same set as analyzed previously with LIBS. 


\subsection{LA-ICP-MS Experimental Instrumentation for Glass Analysis}

All samples were examined on the non-float surfaces of the respective glass. Float glass certified reference material (CRM) NIST 1831 was included into the experimental procedure as an internal control to determine accuracy, because of the similar matrix.

All glass analysis was conducted on a quadrupole ELAN DRC II (Perkin Elmer, Shelton, CT, USA) to a $213 \mathrm{~nm} 4$ nanosecond Q-switched Nd:YAG laser (NewWave Research, Fremont, CA, USA). The laser was operated at a fluence of $27 \mathrm{~J} / \mathrm{cm}^{2}$, with a spot size of $55 \mu \mathrm{m}$, a repetition rate of $10 \mathrm{~Hz}$, a 60 second dwell time and single spot ablation. Helium gas, at flow rate of $0.9 \mathrm{~L} / \mathrm{min}$, was the carrier gas from the sample chamber which blended with argon, the makeup gas, at $1 \mathrm{~L} / \mathrm{min}$ before introduction into the ICP. The RF power of the ICP was set to $1500 \mathrm{~W}$, with the plasma is being sustained with an argon flow of $16 \mathrm{~L} / \mathrm{min}$. 


\subsection{Statistical Analysis}

The LA-ICP-MS analyses of the glass samples were performed following the NITECRIME (Natural Isotopes and Trace Elements in Criminalistics and Environmental Forensics) method ${ }^{174}$. The isotopic elemental menu was comprised of ${ }^{7} \mathrm{Li},{ }^{25} \mathrm{Mg},{ }^{27} \mathrm{Al}$, ${ }^{29} \mathrm{Si},{ }^{39} \mathrm{~K},{ }^{42} \mathrm{Ca},{ }^{49} \mathrm{Ti},{ }^{55} \mathrm{Mn},{ }^{57} \mathrm{Fe},{ }^{85} \mathrm{Rb},{ }^{88} \mathrm{Sr},{ }^{90} \mathrm{Zr},{ }^{118} \mathrm{Sn},{ }^{137} \mathrm{Ba},{ }^{139} \mathrm{La},{ }^{140} \mathrm{Ce},{ }^{146} \mathrm{Nd},{ }^{178} \mathrm{Hf}$, ${ }^{206} \mathrm{~Pb},{ }^{207} \mathrm{~Pb}$, and ${ }^{208} \mathrm{~Pb}$. The CRM NIST 612 glass was used as an external calibrator and ${ }^{29} \mathrm{Si}$ was used as the internal standard. The CRM NIST 612 glass was analyzed at the beginning, end and throughout the experiment to account for drift over the time of the analysis. All glass samples were analyzed 3-4 times at different locations to ensure representative sampling and to account for any inherent sample heterogeneity.

The integrated signal was background subtracted and Glitter Software (GEMOC v4.4, Macquarie University, Australia) was used to determine the concentration of the elements. Data analysis was done with analysis of variance (ANOVA) pairwise comparisons with the General Linear Model (GLM) with Tukeys's honestly significant different test (HSD) using Systat 11 data analysis software (San Jose, CA). T-tests using Microsoft Excel (Microsoft, Redmond, WA) at a 95\% confidence interval were performed to glass samples found indistinguishable, having very similar elemental profiles. 


\subsection{Discrimination Results}

The sample set produced 351 possible pairs. Two duplicate samples were analyzed at random times during the analysis for method validation. The duplicate samples were positive controls and were required to be indistinguishable from itself. The glass standard NIST 1831 was used to determine accuracy.

Float glass samples ranged from years apart to days apart. As predicted the float glass samples that were further apart in manufacturing date produced a greater variation in their elemental profile. All possible pairs were discriminated from one another except for the two positive controls, which were found indistinguishable by the elemental menu. Table 14 provides a list of the top discriminating isotopes and the percent each isotope distinguished. The highest discriminating elements in this sample set were Ti, Fe and $\mathrm{Zr}$.

Table 16. LA-ICP-MS Discriminating Isotopes and Percent Discrimination for Pilkington America Inc.

\begin{tabular}{|c|c|c|}
\hline Element & \# pairs & $\%$ discriminated \\
\hline${ }^{49} \mathrm{Ti}$ & 325 & 92.6 \\
\hline${ }^{57} \mathrm{Fe}$ & 282 & 80.3 \\
\hline${ }^{90} \mathrm{Zr}$ & 281 & 80.1 \\
\hline${ }^{39} \mathrm{~K}$ & 242 & 68.9 \\
\hline${ }^{88} \mathrm{Sr}$ & 223 & 63.5 \\
\hline${ }^{55} \mathrm{Mn}$ & 206 & 58.7 \\
\hline${ }^{27} \mathrm{Al}$ & 108 & 30.8 \\
\hline $\begin{array}{l}178 \\
\text { Hf }\end{array}$ & 33 & 9.4 \\
\hline${ }^{137} \mathrm{Ba}$ & 26 & 7.4 \\
\hline${ }^{25} \mathrm{Mg}$ & 7 & 2.0 \\
\hline${ }^{140} \mathrm{Ce}$ & 4 & 1.1 \\
\hline
\end{tabular}


Generally stating, the further apart in time float glass is manufactured, the greater the variability is witnessed in the elemental profile. For example, pair 12-28-08 and 0214-10 were manufactured $\sim 1$ year apart and differed by 4 elements: Ti, Mn, $\mathrm{Sr}$ and $\mathrm{Ba}$. Pairs manufactured within the same month $(02 / 07 / 10,02 / 14 / 10,02 / 18 / 10$ and $02 / 25 / 10)$ differed from one to three elements. Pairs 02/14/10 and 02/18/10 manufactured four days apart vary only in $\mathrm{Sr}$ and $\mathrm{Ti}$ concentration.

Table 15 lists the excel output for a t-test assuming unequal variances for sample pairs $12 / 26 / 08$ and 12/28/08 which were manufactured 2 days apart and vary by only one element, Ti. Table 15 also lists the excel output for two duplicate samples, P031910 and PD031910 which were analyzed at random times throughout the experimental procedure. The t-tests were conducted at a 95\% confidence interval. Defining the null as hypothesis as there is no significant difference, if the $\mathrm{P}$ value is greater than 0.05 , the null hypothesis is accepted. Sample pairs P031910 and PD031910 produce a P value of approximately 0.41 , concluding that there is no significant difference between these two samples. The results validate that the method is producing no Type I errors, false exclusions.

Sample pairs $12 / 26 / 08$ and $12 / 28 / 08$ produced a $\mathrm{P}$ value of 0.0007 . With a $\mathrm{P}$ value less than 0.05 the null hypothesis is rejected and these two samples are found to be significantly different. The element concentration for Ti determined by LA-ICP-MS was $952.57 \pm 20.64 \mathrm{ppm}$ (RSD 2.17\%) and 862.25 \pm 12.89 (RSD 1.50\%) for 12/26/08 and $12 / 28 / 08$, respectively. These concentrations are close to one another, demonstrating that a sensitive, analytical technique is needed for the forensic discrimination of glass. 
Table 17. Excel Output for T-test Assuming Unequal Variances for Duplicate and Sample Pairs Manufactured Two Days Apart

\begin{tabular}{|c|c|c|c|c|c|}
\hline${ }^{49} \mathrm{Ti}$ & $P 031910$ & $P D 031910$ & ${ }^{49} \mathrm{Ti}$ & P122608 & $P 122808$ \\
\hline Mean & 284.56 & 278.40 & Mean & 952.57 & 862.25 \\
\hline Std. Dev. & 11.3 & 7.6 & Std. Dev. & 20.6 & 12.8 \\
\hline Observations & 4 & 4 & Observations & 4 & 4 \\
\hline df & 5 & & df & 5 & \\
\hline t Stat & 0.91 & & t Stat & 7.42 & \\
\hline$P(1<=t)$ & & & $P(1<=t)$ & & \\
\hline two-tail & 0.4065 & & two-tail & 0.0007 & \\
\hline $\begin{array}{l}\text { t Critical two- } \\
\text { tail }\end{array}$ & 2.57 & & $\begin{array}{l}\text { t Critical two- } \\
\text { tail }\end{array}$ & 2.57 & \\
\hline
\end{tabular}

It is possible that glass fragments originating from the same source can be discriminated as a result of the difference in precision of measurement of the instrumentation and precision of the glass pane. For example, if the precision of the measurement for a given fragment is smaller than the overall precision of elemental concentrations within the glass pane, the sample would be discriminated. Therefore, it is important that the forensic examiner provides a representative characterization of the elemental profile to account for the homogeneity/heterogeneity of the glass pane to prevent incorrect associations or discriminations. 


\subsection{Conclusions}

LA-ICP-MS is shown to be a sensitive analytical method for the forensic association and discrimination of float glass. The temporal variation in the elemental concentration of float glass within a single manufacturing plant was determined for the first time. The variations of these elemental profiles provide $100 \%$ discrimination of samples within the manufacturing plant.

Variations in the trace and minor elements, $\mathrm{Zr}, \mathrm{Sr}, \mathrm{Mn}, \mathrm{Fe}$ and Ti, produced the greatest power of discrimination. The results of this study corroborate previous studies that report float glass samples from different manufacturing plants are significantly different by chemical composition and can be discriminated by many elements. The elemental composition of float glass originating from a single manufacturing plant is most similar within a month's production time. Greater variability in the elemental profile is observed in single plant production, as the manufacture dates increase in time. The greatest variability is observed between different float glass manufacturers. 


\section{Quantitative Analysis of Liquids from Aerosols and Microdrops using Laser Induced Breakdown Spectroscopy}

Inkjet printing will be used to deliver to deliver small sub-nanoliter volume solutions with known absolute mass loadings for LIBS analysis. Inkjet printing is a contactless method for depositing precise volumes of solutions and able to deliver drops ranging from a few microns in diameter to tenths of a millimeter ${ }^{62}$, depending on the nozzle orifice.

It is the motivation of this research to use LIBS to accurately and precisely analyze small volumes of solution, $\leq 300 \mu \mathrm{L}$ for aerosol analysis and $90 \mathrm{pL}$ for singlemicrodrops. These two methods eliminate the splashing and the sample preparation steps that have been reported for previous LIBS solution methods, as well as dramatically reduce the total volume needed for analysis.

\subsection{The LIBS Instrumentation}

A schematic of the LIBS instrumentation can be seen in Figure 30. The LIBS system was constructed using a Solo Nd:YAG PIV dual head laser (New Wave Research, Freemont California, USA) operated at $532 \mathrm{~nm}$ for single-pulse and collinear double-pulse experiments for aerosol and single microdrop in air. Microdrop LIBS on the aluminum stub utilized a 266 nm Tempest Laser (New Wave Research, Freemont California, USA). Pulse widths of both laser systems were 3-5 ns full width half-maximum with a beam diameter of approximately $4 \mathrm{~mm}$ exiting the laser head. Flashlamps and Q-switches were both externally controlled by a Berkeley Nucleonics (San Rafael, California, USA) pulse/delay generator model 575. A Galilean telescope was used to enlarge the beams 
approximately three times to $12 \mathrm{~mm}$ and were then focused to a spot size $\sim 85 \mu \mathrm{m}$ for aerosol and microdrop in air and $\sim 215 \mu \mathrm{m}$ for microdrops on an aluminum stub, through a plano-convex lens with a focal length of $150 \mathrm{~mm}$. The LIBS optical emissions were collected and imaged at a 90 degree angle from the plasma using a pair of plano-convex lenses with a focal distance of $75 \mathrm{~mm}$ onto a $50 \mu \mathrm{m}$ fiber optic cable that was coupled to the entrance slit of an AndorMechelle 5000 spectrometer equipped with an Ando iStar intensified CCD camera using a 1024 x 1024 chip (Andor Technology, South Windsor, CT, USA). Solis software (Andor Technology) was used to control the gate delay, gate width and integration time. Five replicates were analyzed and averaged for the calculations.

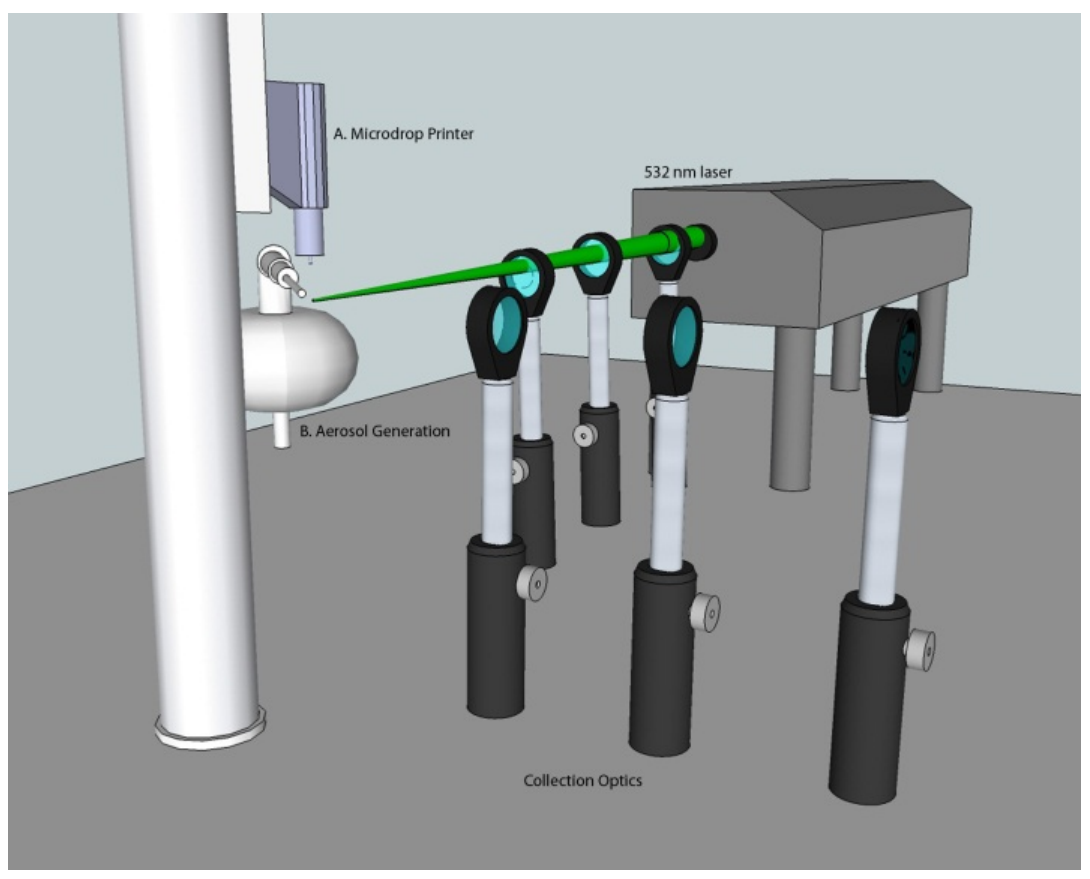

Figure 30. Schematic of a) Microdrop LIBS in air or b) Aerosol LIBS experimental set-up 


\subsection{LIBS Optimization for Aerosol and Microdrop analysis}

Optimum gate delays and gate widths depended on the emission line, sample matrix, laser fluence, and the ambient atmosphere. Temporally resolved measurements are evaluated to enhance the emissions lines of the LIBS spectrum. As vast amount of research has been done on temporal gating ${ }^{175-178}$ and by utilizing the different relaxation times between lines, spectral interferences may be minimized ${ }^{179}$. Laser energies, gate widths, gate delays (respective to laser pulse) and the interpulse delay were optimized for highest signal-to-noise, corresponding to the best precision and accuracy. It is the goal of the optimization studies to produce the highest emission intensities for the desired analyte. As a result of all optimization studies being conducted with similar experimental procedure, repetition is avoided by only illustrating particular studies.

The plasma is formed within nanoseconds after the pulse but the detector reading is delayed approximately $1 \mu \mathrm{s}$, thereby allowing the continuum to decay. The continuum is primarily because of the Bremsstrahlung process in which is dominated by photons emitted by electrons ${ }^{72}$ and this process decays more quickly with time than the spectral lines. For the current work, the plasma was viewed from sequential gate delay times ranging from $1 \mu \mathrm{s}$ to $3.5 \mu \mathrm{s}$ with a constant acquisition time of $10 \mu \mathrm{s}$.

Figure 31 illustrates a linear relationship between the gate delay time and the intensity values. The longer the gate delay the less spectral emission is detected. 

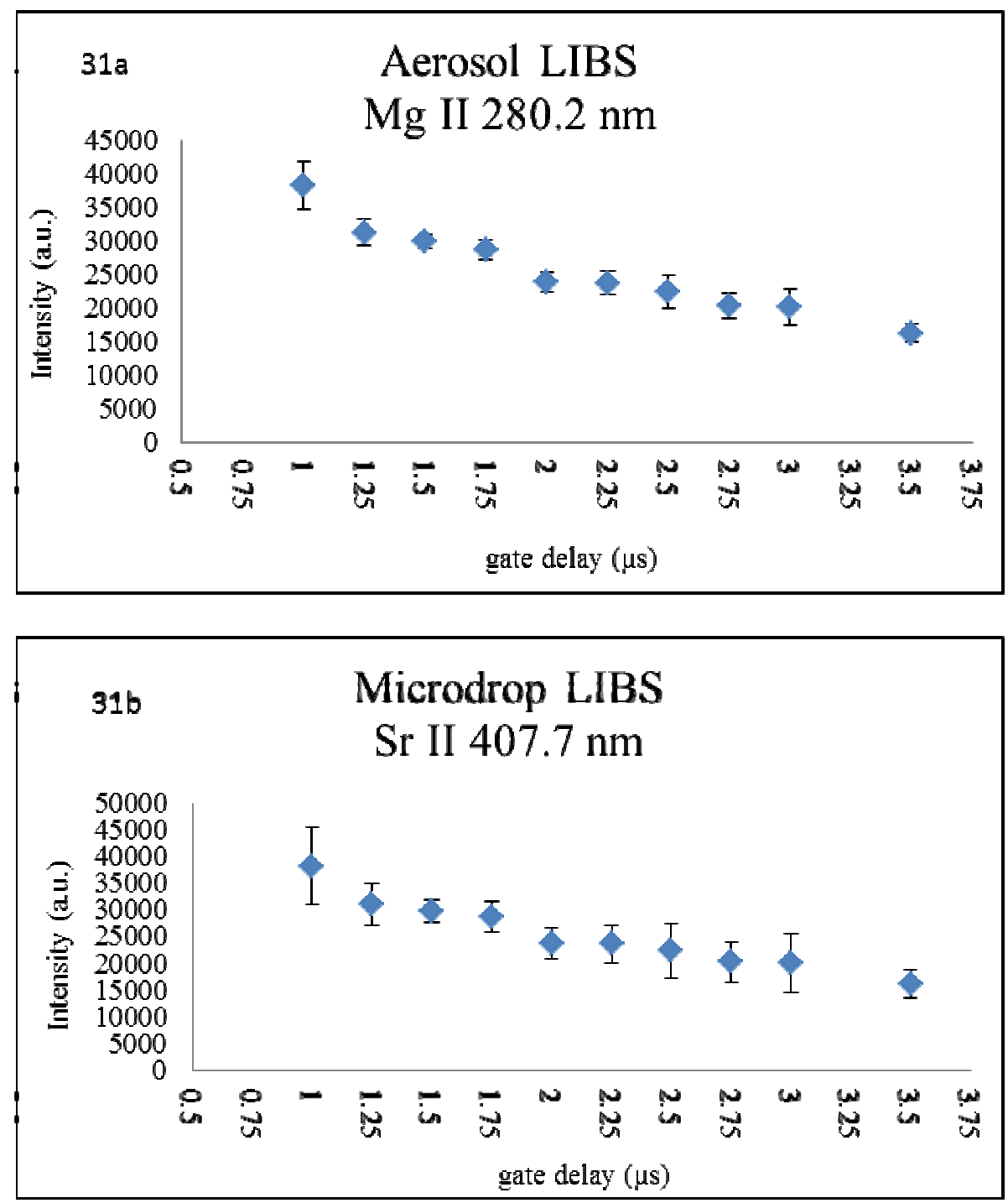

Figure 31. Optimization of gate delay for a) Aerosol and b) Microdrop LIBS in air

A gate delay of $1.75 \mu \mathrm{s}$ in both aerosol and microdrop LIBS was determined to provide the best precision with maximum intensity, after background subtraction. It is at this point that the light is collected, transferred and detected. Each element has an exclusive emission spectrum; the wavelength corresponding to the emission line.

A precision of $\sim 5 \%$ RSD was obtained in aerosol LIBS for gate delays between 1.75 and $3.50 \mu$ s, while a gate delay of $1 \mu$ s produced a precision of $9 \%$ RSD. The precision in microdrop LIBS was not as good as aerosol LIBS. The average precision 
was $\sim 13 \%$ RSD. Both configurations had worse precision at the $1 \mu$ s time delay. This is attributed to the continuum still being dominant and not having achieved local thermodynamic equilibrium.

Detector observation times (gate width) vary greatly from nanoseconds to hundreds of microseconds, depending on the application, matrix and laser energy. There are different rates of decay for the continuum and the analyte species. Below in Figure 32 is an example of the optimization for the detector observation time for collinear doublepulse LIBS.

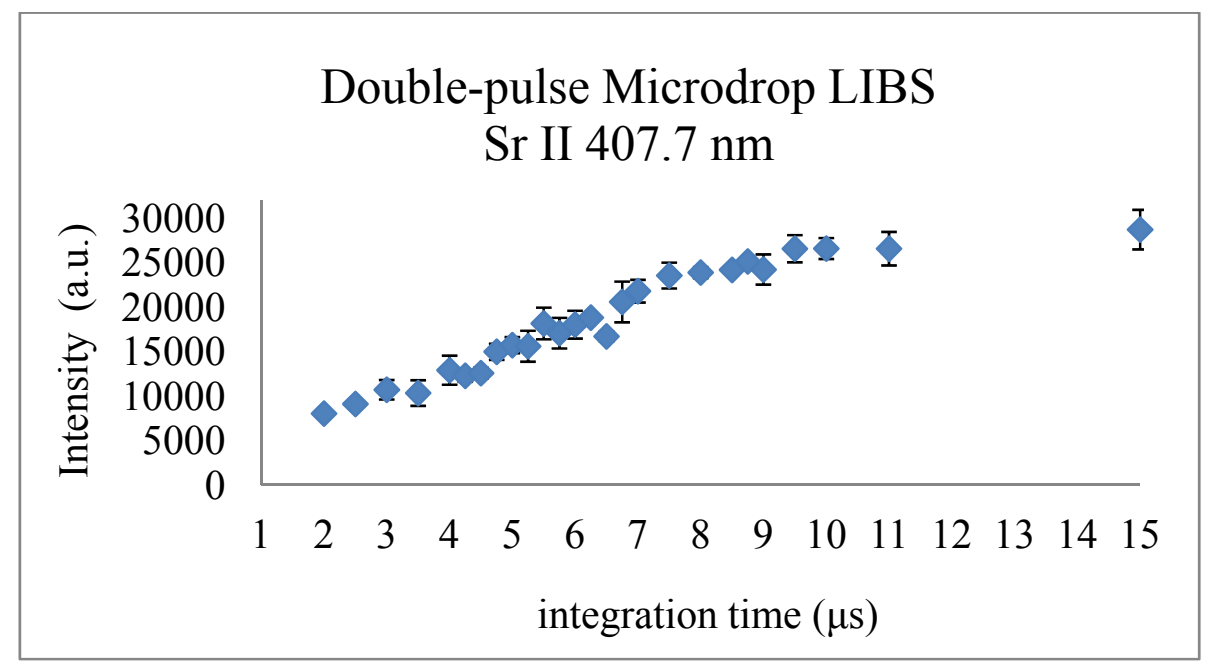

Figure 32. Optimization of integration time for double-pulse microdrop LIBS

It is observed in this figure that the intensity does not increase significantly after $10 \mu \mathrm{s}$. The integration time of $10 \mu$ s was determined to be the optimized value.

For the current work, a constant high signal corresponding to the best sensitivity and repeatability was found at an observation time of $8.75 \mu$ s for double-pulse and $6.75 \mu \mathrm{s}$ for single-pulse with an average precision in the double-pulse configuration of $3.5 \%$ RSD for aerosol LIBS. Microdrop LIBS observation times were $7 \mu$ s for single-pulse and $10 \mu \mathrm{s}$ for double-pulse corresponding to a $14 \%$ RSD for microdrop LIBS. 
It has been noted that conventional single-pulse LIBS is less sensitive than the competing atomic spectroscopic technique of ICP-OES that utilizes a sustained plasma as the excitation source but also requires much more analytical volume. In an effort to increase the LIBS sensitivity, double-pulse LIBS excitation is explored. Different laser pulse configurations have contributed to improving the limits of detection (LOD), by increasing the LIBS signal ${ }^{63,64}$. Double-pulse LIBS techniques use a combination of two lasers or two pulses from one laser. Both are spatially overlapped and the two laser pulses are focused and separated by an optimized time ranging from nanoseconds to tens of microseconds separation.

The current work in aerosol and microdrop LIBS uses a collinear double-pulse configuration (as opposed to an orthogonal configuration). In the collinear configuration the two laser pulses propagate sequentially along the same axis and are focused onto the same location on the sample. Collinear double-pulse LIBS leads to increased mass ablation and the reheating of the plasma with the second pulse producing a longer-lived plasma than the single-pulse, increasing the analytical signal ${ }^{63,64}$. The separation of the two laser pulses was optimized for the highest increase in analytical signal. Figure 33 for microdrop LIBS illustrates the analytical signal trend for an interpulse laser delay ranging from zero to 3000 nanoseconds. 


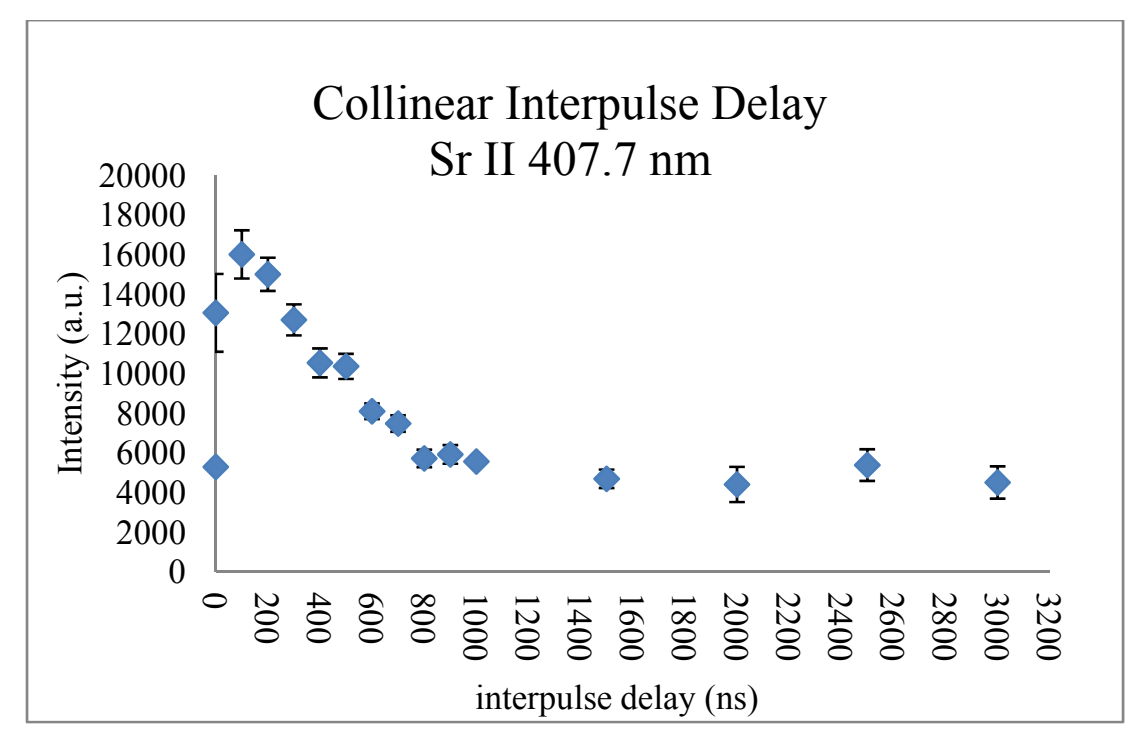

Figure 33. Collinear interpulse delay optimization in aerosol LIBS for Sr II $407.7 \mathrm{~nm}$

The lowest point on the graph at time delay zero is for single-pulse LIBS and it produced a background subtracted intensity of 5285 counts. The mid-point on the graph at time delay of zero is the time when both pulses are being delivered with no delay; this produced an intensity of 13053 counts. The highest point in the graph is at $100 \mathrm{~ns}$ with a produced intensity value of 16002 counts. This was a preliminary study to observe the trend of collinear pulse in an aqueous environment, to determine approximately when the greatest intensity increase is observed. A further study was then conducted with microdrop LIBS to focus in on the exact optimized delay.

The collinear interpulse delay is optimized for the highest emission signal and best precision for the elements of interest. Figure 34 illustrates the correlation of intensity with interpulse delay in intervals of $50 \mathrm{~ns}$ for four different emission lines. It is observed that at greater delay times the plasma density decreases with the gate width held constant. Each elemental emission line has two points on the y-axis at time zero. The lower point is single-pulse LIBS and the higher point is both lasers firing at the same time 
with no interpulse delay. The same trend as previously was observed, however, the highest analytical signal was observed at an interpulse delay of $50 \mathrm{~ns}$. The highest increase observed for emission lines Sr II $421.55 \mathrm{~nm}, \mathrm{Mg}$ II $280.27 \mathrm{~nm}$, Ba II $455.40 \mathrm{~nm}$ and $\mathrm{Ca} 396.85 \mathrm{~nm}$ was during the interpulse delay time of $50 \mathrm{~ns}$. Both aerosol and microdrop LIBS produced similar results, with the greatest increase in analytical signal corresponding to an interpulse delay of $50 \mathrm{~ns}$.

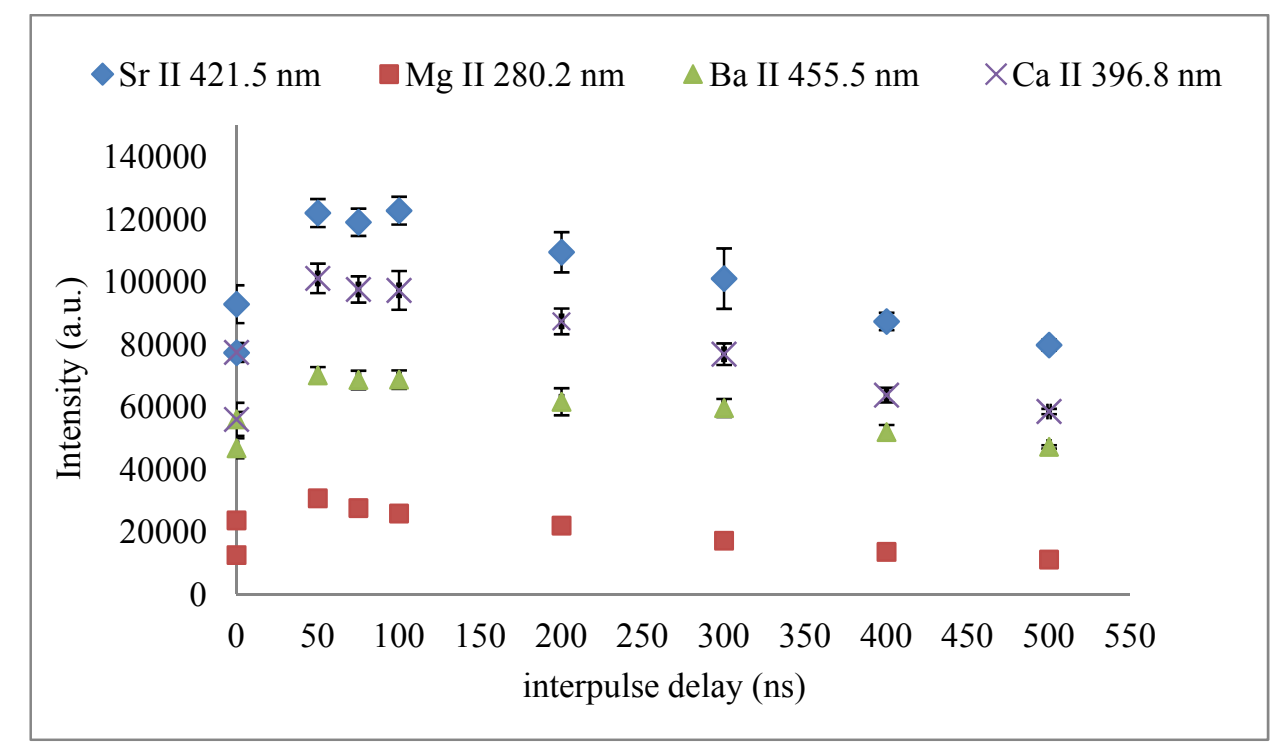

Figure 34. Collinear interpulse delay optimization in microdrop LIBS for $\mathrm{Sr}$ II $421.5 \mathrm{~nm}$, Mg II 280.2 nm, Ba II $455.5 \mathrm{~nm}$ and Ca II $396.8 \mathrm{~nm}$

Argon has been demonstrated to increase the LIBS signal by producing a higher plasma temperature and higher electron density, resulting in higher emission intensity. Iida demonstrated that the plasma decay was reduced in an argon environment because of its low thermal conductivity ${ }^{180}$. Wisbrun et al. demonstrated that an argon atmosphere produced almost doubled the intensity values and provided better precision. This was contributed to argon protecting the excited atoms from forming more stable compounds, such as oxides. ${ }^{181}$ Argon was used as the carrier gas at a constant flow of $700 \mathrm{~mL} / \mathrm{min}$ to 
transport the solution that was converted to a fine aerosol by a commercial nebulizer (ESI, Omaha, NE, USA) designed for ICP systems with a flow rate of $40 \mu \mathrm{L} / \mathrm{min}$. Figure 35 shows the optimization studies for the flow of Argon.
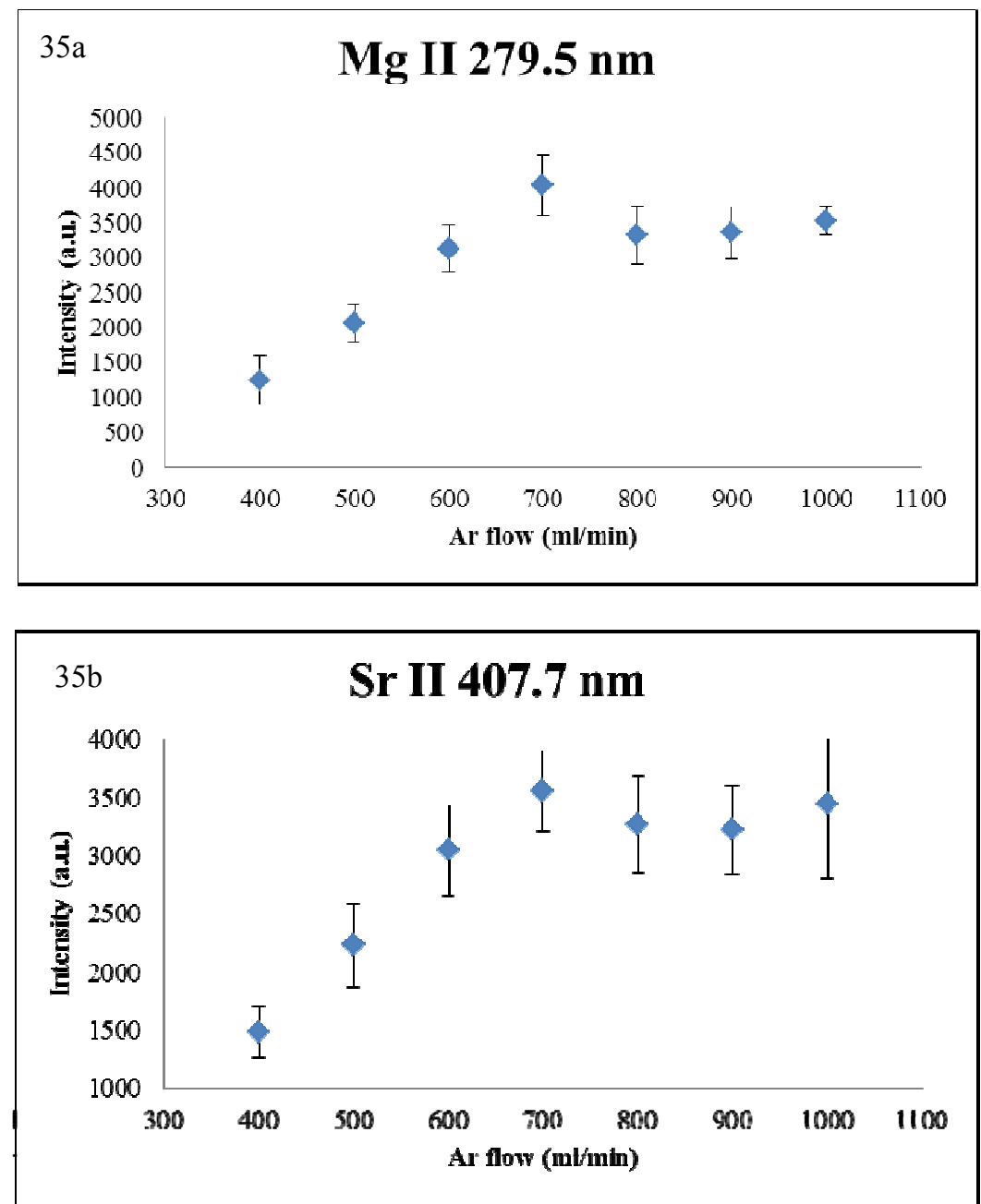

Figure 35. Optimized argon flow rate for aerosol LIBS for a) Mg II 279.5 and b) Sr II $407.7 \mathrm{~nm}$ It is observed that there is a linear relationship between argon flow rates and intensity between the flows of 400 and $700 \mathrm{~mL} / \mathrm{min}$. The flow rate of $700 \mathrm{~mL} / \mathrm{min}$ was chosen as it provided the highest analytical signal.

In conclusion, the optimized parameters, for single-pulse aerosol LIBS were a laser wavelength of $532 \mathrm{~nm}$, laser energy at $65 \mathrm{~mJ}$, a gate delay of $1.75 \mu \mathrm{s}$, a gate width 
of $6.75 \mu \mathrm{s}$ and an argon flow of $700 \mathrm{~mL} / \mathrm{min}$. Double-pulse aerosol LIBS parameters had both pulses delivered at $65 \mathrm{~mJ}$, with an interpulse delay of $50 \mathrm{~ns}$, a gate width of $8.75 \mu \mathrm{s}$ and all other settings remained unchanged.

The optimized parameters for single pulse microdrop LIBS on an aluminum stub were a laser wavelength of $266 \mathrm{~nm}$, a gate delay of $1.1 \mu \mathrm{s}$, a gate width of 5.75 seconds and a frequency of $0.66 \mathrm{~Hz}$.

The optimized parameters for single-pulse and collinear double-pulse microdrop LIBS experiments were, for single-pulse: a laser wavelength of $532 \mathrm{~nm}$, a laser energy for $35 \mathrm{~mJ}$ per pulse, an argon sheath over the LIBS plasma delivered at $300 \mathrm{ml} / \mathrm{min}$ with $10 \mathrm{~mm}$ inner diameter tubing positioned $60 \mathrm{~mm}$ from the LIBS plasma, a gate delay of $1.75 \mu$ s (respective to laser pulse), gate width of $7 \mu \mathrm{s}$, a frequency of $0.66 \mathrm{~Hz}$. Collinear double-pulse parameters consisted of both pulses at $35 \mathrm{~mJ}$, with an interpulse delay of 50 ns and a gate width of $10 \mu \mathrm{s}$, while all other parameters remained constant. Precision and accuracy was measured by 5 replicate measurements on all samples. 


\subsection{Aerosol LIBS Experimental Instrumentation}

An injector tube, typically used for the torch of an ICP-MS, was attached to the flow chamber and produced a fine aerosol mist. The laser was focused $\sim 2 \mathrm{~mm}$ from the injector orifice, where the effluent was introduced into the LIBS plasma. Figure 36 is a photograph taken during an aerosol LIBS event. At the tip of the injector, the LIBS plasma ionizes the effluent from the injector.

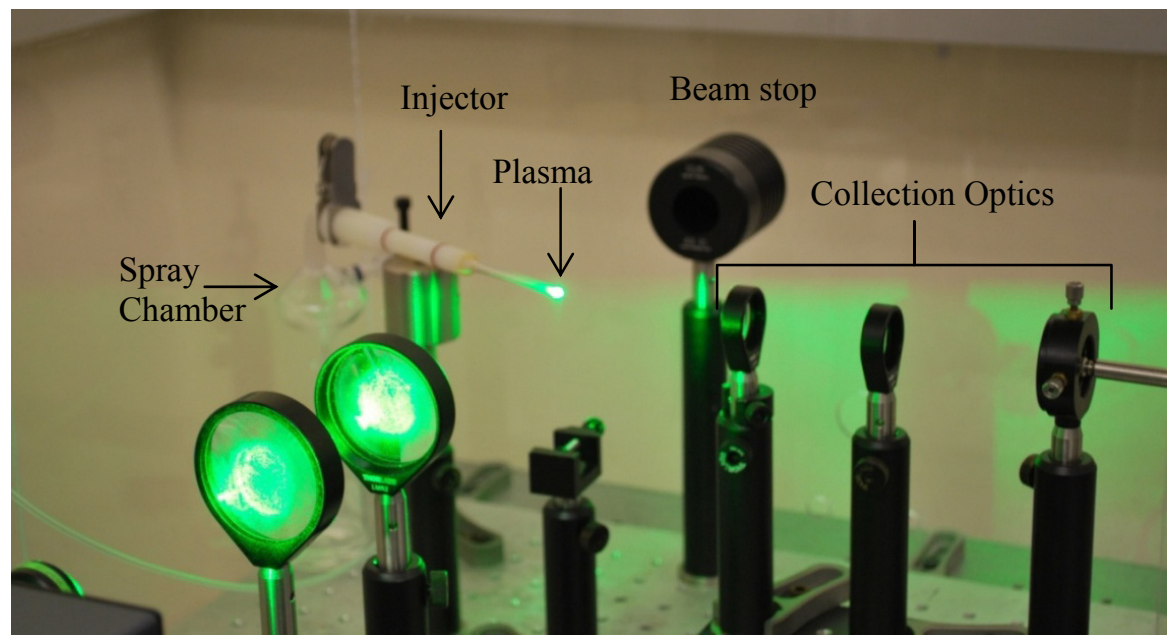

Figure 36. Experimental aerosol LIBS setup

The LIBS signal is then collected from the side with the collection optics where the signal is then transformed and output onto the computer.

Single-pulse and collinear double-pulse experiments were achieved with a 20 shot accumulation at a frequency of $0.66 \mathrm{~Hz}$, which equates to a 30 second analysis time, and an absolute volume of $20 \mu \mathrm{L}$ being consumed per analysis.

Multi-element calibration standards were prepared from stock 1000 and 10,000 ppm solutions and made into concentrations ranging from 0 to $3000 \mathrm{ppm}$. For aerosol LIBS, the multi-element solutions containing $\mathrm{Ca}, \mathrm{Ba}, \mathrm{Sr}$ and $\mathrm{Mg}$ were prepared with final concentrations of $0,5,7.5,15,25,50,125$, and $250 \mathrm{ppm}$. Continuing calibration 
verification standards (CCVs) were prepared independently and used for method validation and to quantify bias at $25 \mathrm{ppm}$ for aerosol analysis. The CCVs were analyzed randomly throughout the experimental procedure. 


\subsection{Aerosol LIBS Data}

For aerosol LIBS, the use of the spray chamber created uniform sized droplets producing better precision. The ejected particles are captured by the plasma. Images acquired using a PI-MAX nanosecond gated camera (Princeton Instruments, Acton, MA, USA) suggests that not all the effluent exiting the injector was being consumed. The plasma is capturing a only certain percentage of the particles ejected by the LIBS plasma, Figure 37.

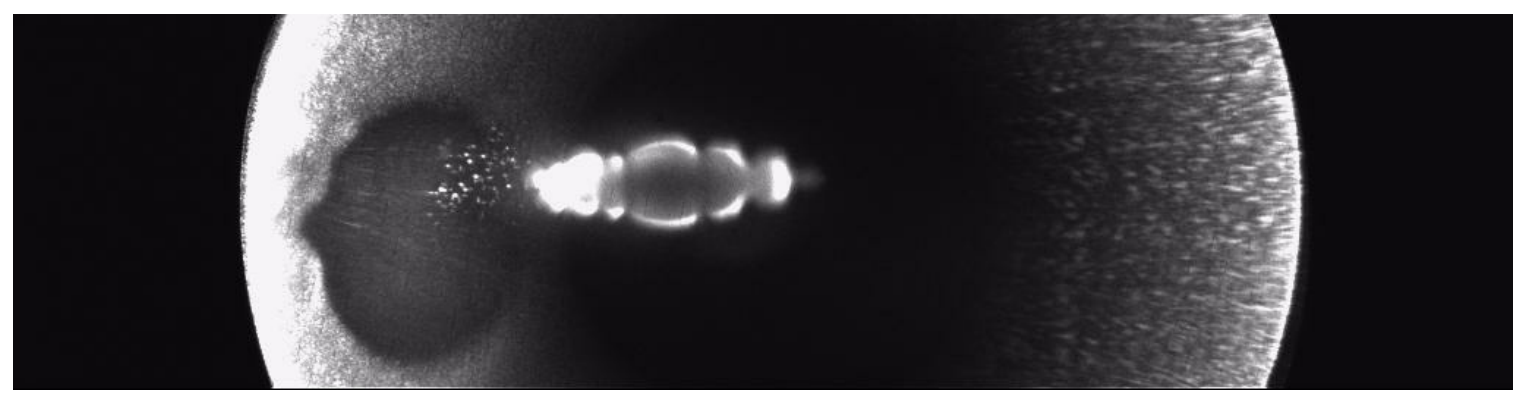

Figure 37. Aerosol LIBS effluent exiting the orifice into LIBS plasma

However, it is expected that a consistent aerosol volume (and analyte mass) of the effluent is consumed within each LIBS plasma formation. Aerosol LIBS analysis consumed less than $300 \mu \mathrm{L}$ of total sample volume during the accumulation of 20 laser pulses per replicate for a total of 5 replicates measurements per sample.

\subsubsection{Aerosol LIBS: Single-pulse and Double-pulse quantitative analysis}

A single-pulse LIBS spectra in Figure 38 illustrates linearly increasing emission line intensities with increasing concentration, indicating LIBS that is capable of quantitative analysis. 


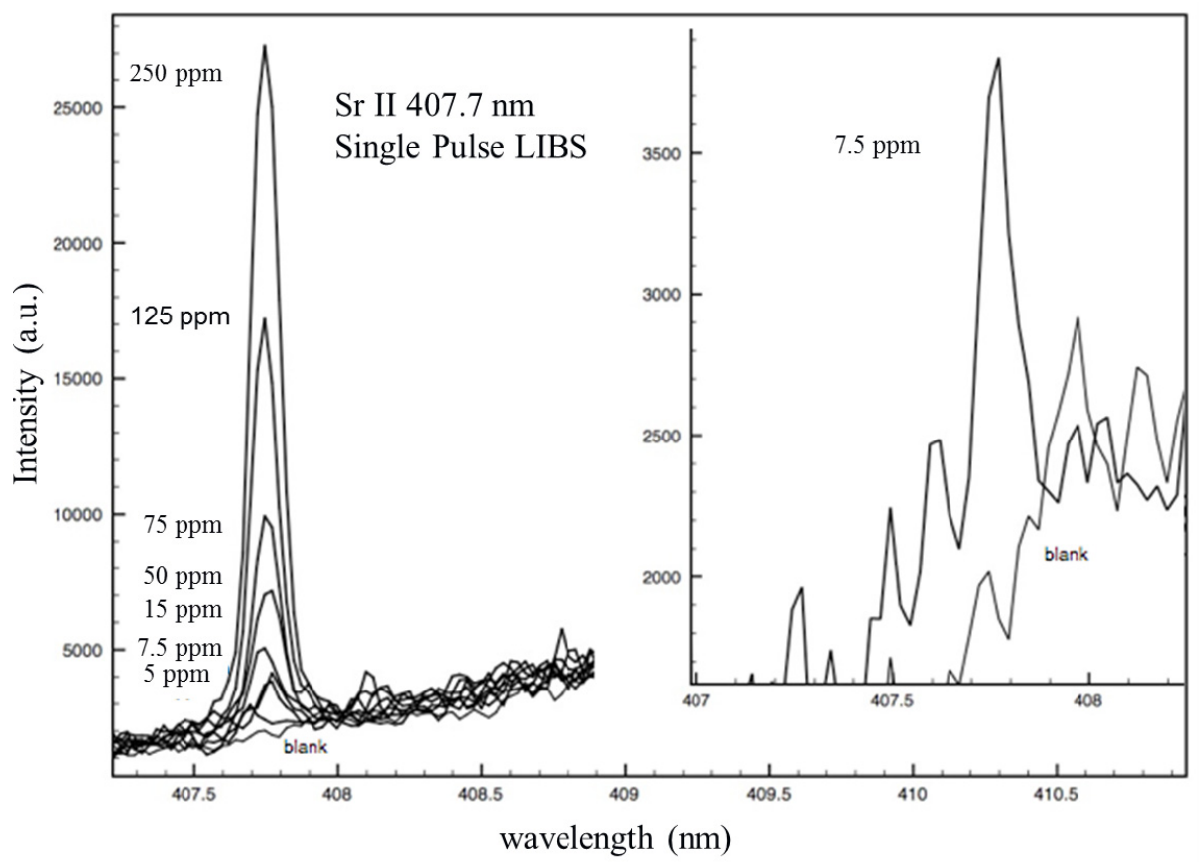

Figure 38. Single-pulse aerosol LIBS spectra for concentrations ranging from 0-250 ppm

In the SP aerosol LIBS spectra seen in Figure 38, it is observed that at a concentration of $7.5 \mathrm{ppm}$, the LOD is approached. Figure 39, seen below, is a double-pulse aerosol LIBS spectrum for $1 \mathrm{ppm}$ and a very nice narrow peak is produced, illustrating the increase in analytical signal, hence lowering the LOD.

The signal enhancements are more pronounced for emission lines with higher excitation energy levels and this observation has previously been reported by others ${ }^{177,178}$. In aerosol LIBS, the sensitivity for the Ca II $396.85 \mathrm{~nm}$ emission line, total analytical volume of $\sim 20 \mu \mathrm{L}$ of a $50 \mathrm{ppm}$ solution ( $\sim 1 \mu \mathrm{g}$ of mass),produced an increase of signalto-noise ratio from 42 in the single-pulse configuration to 62 in double-pulse while improving the precision from $5.2 \% \mathrm{RSD}$ to $0.5 \% \mathrm{RSD}$. 


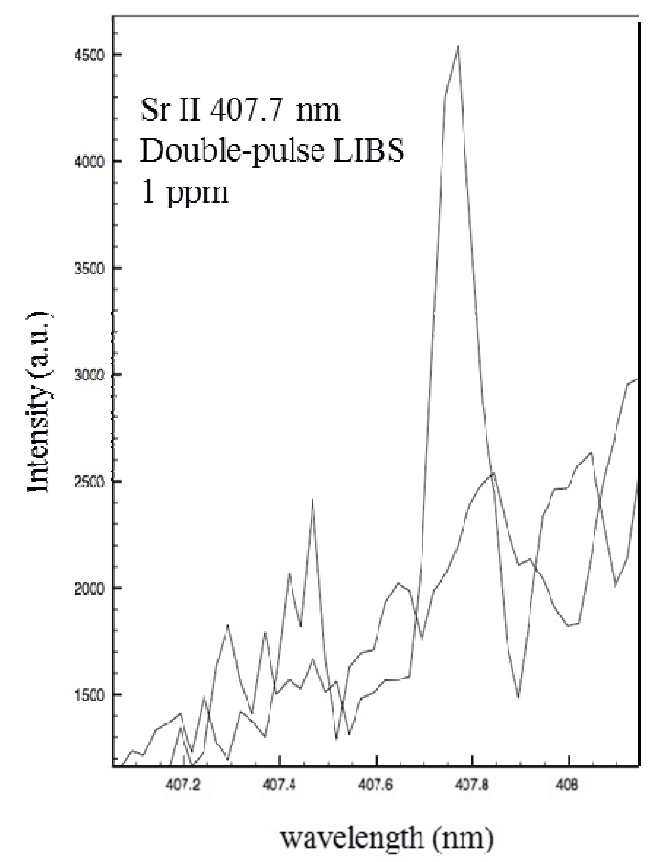

Figure 39. Double-pulse aerosol LIBS spectra of Sr II 407.7nm emission line at a concentration of 1 ppm and blank solution

Previous research has defined the excitation energy level for ionic lines as the sum of the ionization energy and the upper energy level ${ }^{177,182}$. The excitation energy for Ba II 455.40 nm, Sr II $421.5 \mathrm{~nm}$, Ca II $396.9 \mathrm{~nm}$ and Mg II $280.3 \mathrm{~nm}$ are 7.9, 8.6, 9.2, and $12.1 \mathrm{eV}$, respectively. The maximum enhancement in collinear double-pulse LIBS was observed at a $50 \mathrm{~ns}$ interpulse delay. The observed enhancements in aerosol LIBS were a multiple of 1.5, 1.6, 1.7 and 2.5 as compared to a single-pulse for Ba II $455.40 \mathrm{~nm}, \mathrm{Sr}$ II $421.55 \mathrm{~nm}$, Ca $396.85 \mathrm{~nm}$ and Mg II $280.27 \mathrm{~nm}$, respectively, suggesting that the signal enhancements are larger for ionic lines with higher excitation energies.

A calibration of the response versus the concentration was constructed to conduct quantitative analysis for aerosol LIBS. Multi-element solutions containing $\mathrm{Ca}, \mathrm{Ba}, \mathrm{Sr}$ and $\mathrm{Mg}$ with final concentrations of $0,5,7.5,15,25,50,125$, and $250 \mathrm{ppm}$ were used. 
Figure 40 illustrates calibration curves for $\mathrm{Mg}$ and $\mathrm{Ba}$ emission lines by single-pulse aerosol LIBS.
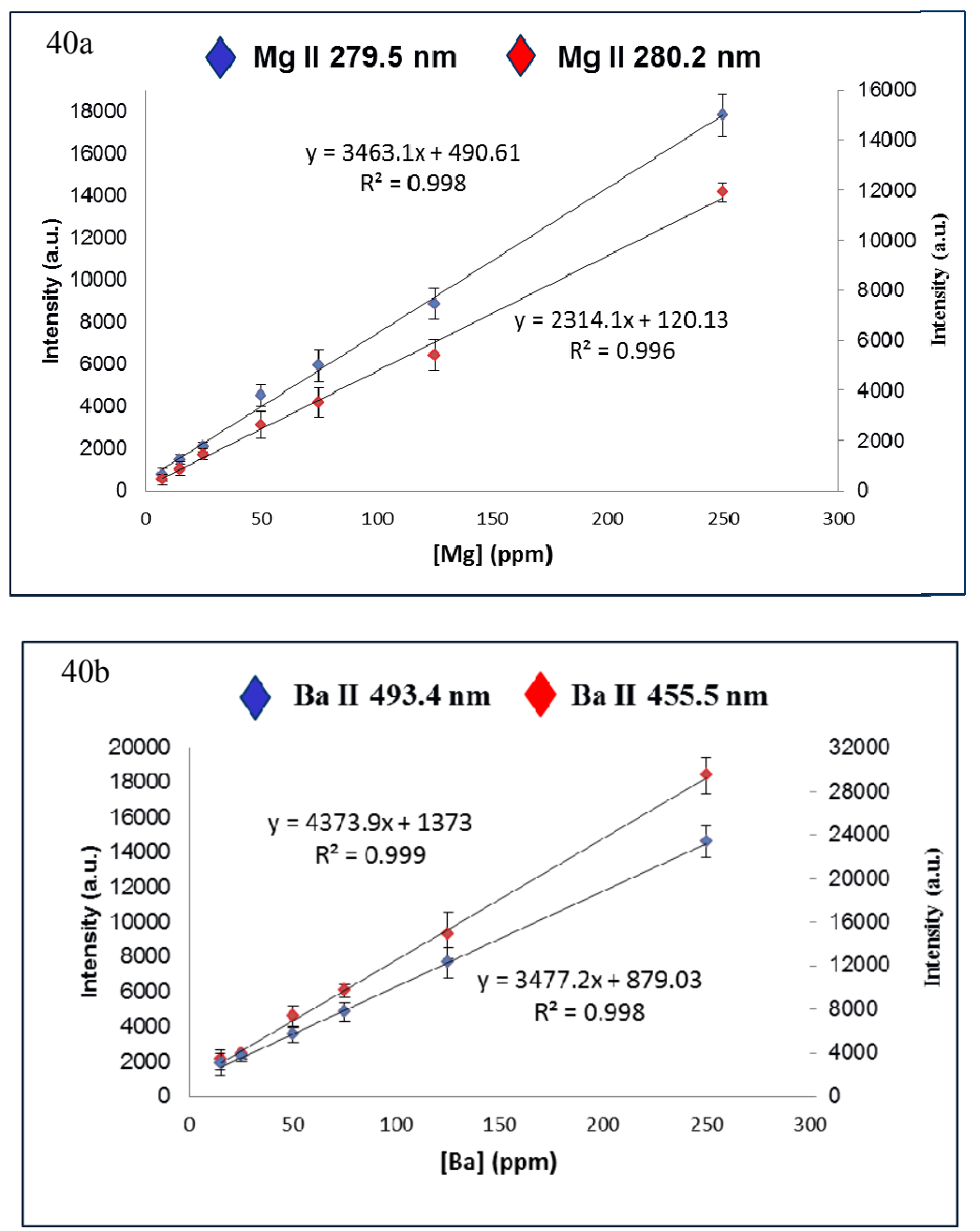

Figure 40. Aerosol LIBS calibration curves for a) Mg II 279.5 and $280.2 \mathrm{~nm}$ b) Ba II 493.4 and 455.5 nm emission lines

As a result of the homoscedasticity of the data, unweighted calibration curves were created for single-pulse and double-pulse LIBS. The LIBS signal response to a known mass concentration was established with good linearity $\left(\mathrm{R}^{2}>0.99\right)$ for both single and double-pulse configurations. All points on the calibration curves of the different emission lines were reproducible with typical precision of 2-6\% RSD. Aerosol LIBS 
produced better sensitivity for the double-pulse versus the single-pulse configuration. The LOD for Sr II $421.5 \mathrm{~nm}$ decreased from $3.3 \mathrm{ppm}$ to $1.0 \mathrm{ppm}$ and Mg II 279.5 decreased from a LOD of 2.6 in single-pulse compared to $0.3 \mathrm{ppm}$ in double-pulse. Ba II $493.4 \mathrm{~nm}$ and Ca II $396.3 \mathrm{~nm}$ produces LODs of $0.7 \mathrm{ppm}$ and $0.6 \mathrm{ppm}$, respectively.

The flow of the nebulizer at $6.67 \times 10^{-7} \mu \mathrm{L} / \mu$ s provided a total analytical volume of $20 \mu \mathrm{L}$ being consumed during the 20 laser pulse analysis. The total mass delivered within the $20 \mu \mathrm{L}$ sample volume at a sample concentration of $25 \mathrm{ppm}$ is $0.05 \mu \mathrm{g}$. With a spectral viewing time totaling $175 \mu \mathrm{s}$, the volume introduced into the plasma during the observation is equivalent to an absolute mass of $2.92 \mathrm{pg}$ consumed per replicate. This is an overestimation, as it is known that the not all of the $20 \mu \mathrm{L}$ effluent into the LIBS plasma is being excited and/or ionized.

Table 18. Figures of Merit for Aerosol LIBS

\begin{tabular}{|c|c|c|c|c|c|c|}
\hline \multicolumn{7}{|c|}{ Aerosol LIBS } \\
\hline $\begin{array}{c}\text { Emission } \\
\text { Line }\end{array}$ & $\begin{array}{c}\text { Conc } \\
\text { (ppm) }\end{array}$ & SNR & $\begin{array}{c}\text { Bias } \\
(\%)\end{array}$ & $\begin{array}{c}\text { Precision } \\
(\%)\end{array}$ & $\begin{array}{c}\text { LOD } \\
\text { (ppm) }\end{array}$ & $\begin{array}{c}\text { LOD } \\
\text { (fg) }\end{array}$ \\
\hline Sr II 421 & 25 & 28 & -0.9 & 3.1 & 1 & 120 \\
\hline Mg II 279 & 25 & 111 & 7.1 & 6.7 & 0.3 & 30 \\
\hline Ba II 493 & 25 & 30 & 1.3 & 5.0 & 0.7 & 170 \\
\hline Ca II 396 & 25 & 62 & -2.9 & 0.5 & 0.6 & 70 \\
\hline \hline
\end{tabular}

Analysis of a continuous calibration verification (CCV) standards containing $25 \mu \mathrm{g} / \mathrm{mL}$ produced bias of as low as $0.9 \%$ and $1.3 \%$ for the emission lines of Sr II $421.5 \mathrm{~nm}$ and $\mathrm{Ba}$ II $493.4 \mathrm{~nm}$ and $2.9 \%$ and $6.7 \%$ for Ca II $396.3 \mathrm{~nm}$ and Mg II 279.5, respectively. 
Using the calculated absolute mass for aerosol LIBS, this translates to a LOD of approximately $120 \mathrm{fg}$ for Sr II $421.55 \mathrm{~nm}, 170 \mathrm{fg}$ for Ba II $493.41 \mathrm{~nm}, 70 \mathrm{fg}$ for Ca II $396.3 \mathrm{~nm}$ and reaches as low as $30 \mathrm{fg}$ for $\mathrm{Mg}$ II $279.80 \mathrm{~nm}$. Table 16 summarizes the figures of merit for aerosol LIBS. 


\subsection{Microdrop Delivery Instrumentation}

Two different inkjet printing systems, JetDrive III and Jetlab 4, purchased from Microfab Technologies, Inc. (Plano, TX) were used to conduct the following experiments.

The JetDrive III was an in-laboratory built system with the necessary printing components purchased through Microfab Technologies, Inc. The components consisted of a printhead, LED strobe light, horizontal viewing optics and a Jetserver controller, see Figure 41. The JetDrive III controller, CT-M2-02 was a stand-alone Windows based control program, that had a built in strobe delay and allowed for manipulations of the bipolar waveform.

The printhead was mounted vertically on a 2 inch optical post, which was mounted on a $\mathrm{x}-\mathrm{y}$ micrometer stage. The printhead was coupled by a Luer connection to a disposable $2.5 \mathrm{~mL}$ syringe which provided the negative pressure. The negative pressure provided by the syringe was controlled by adjusting the height to a level adequate to produce the start of a microdrop. The microdrop was visualized with a strobe LED with a $3000 \mu$ s window and horizontal optics equipped with a reticle for calibration and determination of proper microdrop parameters. Drop size was determined by viewing images of the drop from a $90^{\circ}$ angle using simple graphing software to determine the number of pixels per square mm of the image. Using the formula for volume of a sphere, the drop volume was calculated.

Plexiglas ${ }^{\circledR}$ was used to construct two connecting side walls and an upper covering, enclosing the LIBS collection optics and the microdrop printer. 


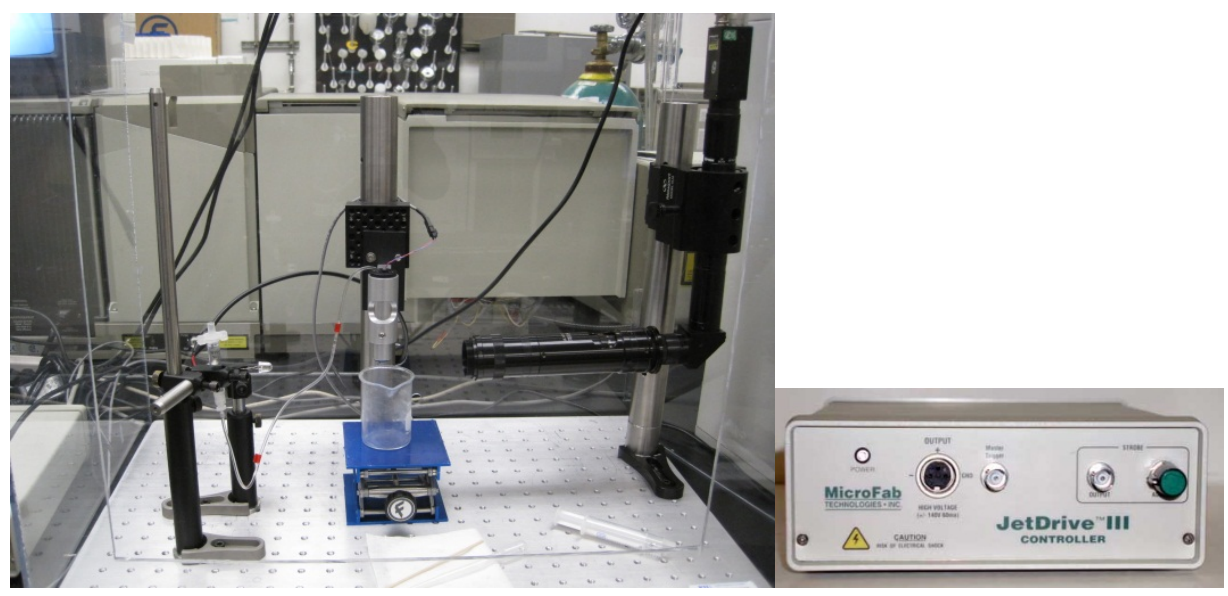

Figure 41. JetDrive III experimental setup and controller

The second system microdrop system used was the Jetlab 4, see Figure 42a. The Jetlab was an upgraded version of the JetDrive III. It provided an automated $x-y-z$ stage, visualization of both the printhead and the substrate to be printed on, see Figure $42 \mathrm{~b}$. This system provided automated pressure control which enhanced the printing stability. The automated stage allowed for patterns to be printed. The updated software was more userfriendly and allowing for the drop images to be calibrated with ease.
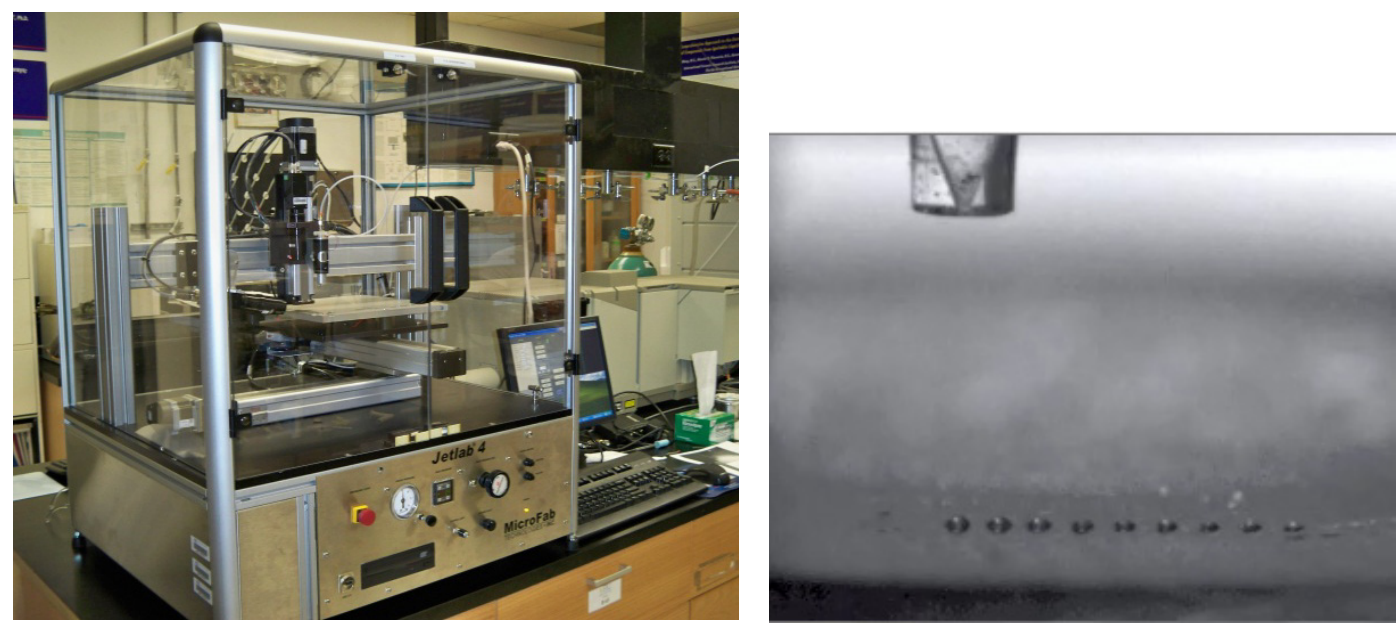

Figure 42. Jetlab 4 a) Microdrop System and b) viewing optics on Al stub substrate Both inkjet systems used a MJ-A style drop-on-demand dispensing device (printhead), see Figure 43, with a Luer connection for PTFE tubing. The dispensing 
devices are suited for aqueous and solvent-based solutions with an orifice diameter of 60 $\mu \mathrm{m}$.

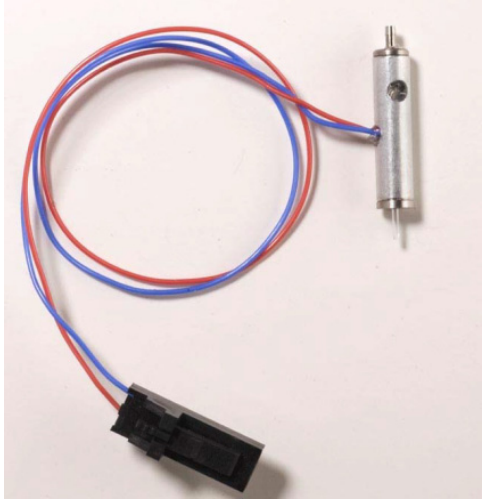

Figure 43. Printhead used for microdrop delivery

The microdrop delivery process is controlled by the drive waveform used for the piezoelectric actuator. Microdrops are produced in both systems by trial and error of the waveform parameters. 


\subsection{Microdrop Printing for LIBS Analysis}

Solid calibration standards can be purchased and LIBS utilizes these standards to determine the relative intensity per concentration of analyte. However, these calibration standards do not allow for absolute mass calibrations or calculations for absolute mass limits of detection. Inkjet printing was utilized through the following experiments to deliver microdrops containing picoliter volumes for LIBS analysis. Knowing the volume of each microdrop permitted for the deposition of known mass quantities of analytes onto the sample surface.

Both configurations on inkjet printing were employed. First, drop-on-demand inkjet printing with the Jetlab 4 instrument was used to deliver known analyte mass quantities for quantification and elemental mapping on aluminum stubs. Inkjet printing allowed for a discrete number of drops, a known absolute mass amount, to be delivered at specified locations.

Continuous mode inkjet printing was employed using the JetDrive III in single microdrop LIBS. A continuous flow of microdrops were produced and timed with the laser pulse for single drop, single laser shot analysis.

\subsubsection{Microdrop Quantification on Aluminum Stub}

The microdrops were deposited onto an aluminum stub (Ted Pella, CA) that were manufactured for SEM analysis. The optimal printing conditions were determined to be a voltage of $+19 /-18 \mathrm{~V}$, a dwell of $12 \mu \mathrm{s}$, an echo of $35 \mu \mathrm{s}$ and the rise and fall times were kept constant at $3 \mu$ s. Dr. Joshi-Kumar aided in the operation of the Jetlab 4. 
Preliminary studies were first conducted on the aluminum stub by LIBS to examine the constituents above the LOD within the elemental profile. The metals chosen for deposition were $\mathrm{Ba}$ and $\mathrm{Sr}$ as these were predetermined by LIBS not to be present in the Al matrix. Multi-element calibration standards were prepared from stock solutions of $1000 \mathrm{ppm}$ and made into a concentration of $250 \mathrm{ppm}$ in $5 \%$ nitric acid consisting of $\mathrm{Ba}$ and Sr. Studies were conducted with analysis of only the Al stub, followed by stub and solvent and finally with the metal solution on Al stub. The metals were deposited into premade craters which were created using a Cetac LSX 500 laser ablation unit (Omaha, NB) equipped with a $266 \mathrm{~nm}$ laser. A spot size of $200 \mu \mathrm{m}$ and 100 shots created a crater $15 \mu \mathrm{m}$ deep with a $200 \mu \mathrm{m}$ diameter, see Figure 44. Figure 44a was produced with a scanning electron microscope, Philips XL 30 (Philips, Eindhoven, The Netherlands). Figure $44 \mathrm{~b}$ and the 3-D images to follow were generated using a Keyence VHS-600 digital microscope (Atlanta, Ga). The craters provided a sample cell for deposition of the microdrops and allowed visualization to accurately aim the laser to the correct location on the sample surface.
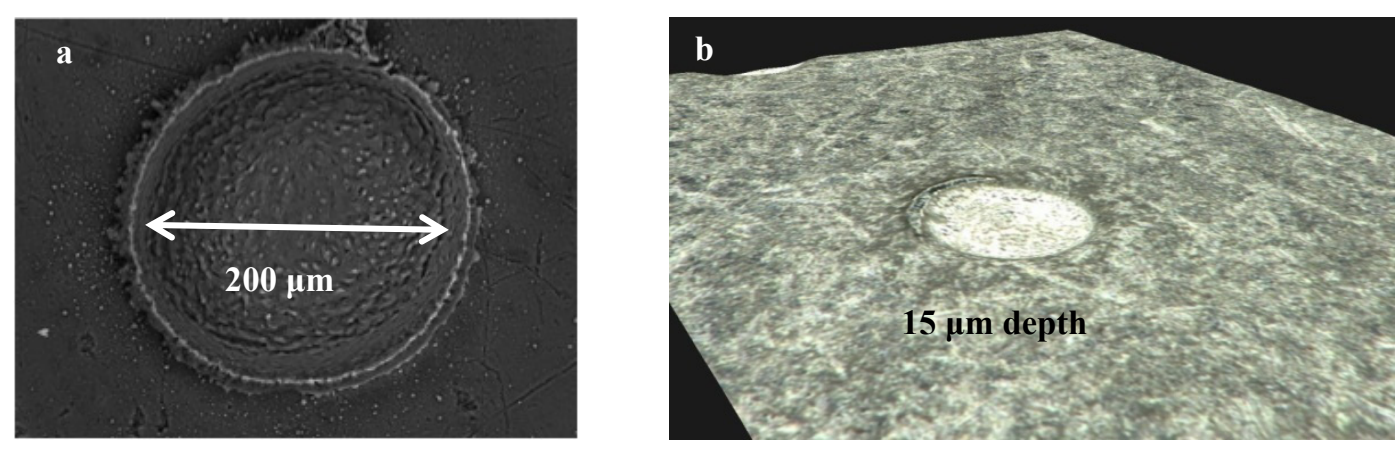

Figure 44. Crater on Al stub pre-LIBS analysis a) $200 \mu \mathrm{m}$ crater on Al stub for microdrop deposition b) Al stub crater with a depth of $15 \mu \mathrm{m}$ for microdrop deposition 
These craters on the aluminum stub were produced having a smaller diameter $(\sim 200 \mu \mathrm{m})$ than that of the laser beam $(\sim 215 \mu \mathrm{m})$ to ensure complete analyte removal. The inkjet was then used to deliver precise mass loadings of the heavy metals into the craters. The visualization software allows for the substrate that is printed on to be observed during the inkjet process. This is used to verify that the drops delivered do not overfill the shallow crater.

To ensure quantitative accuracy it must be assumed that all of the deposited mass is being removed. As can be seen from this single shot analysis spectrum, Figure 45, there is a linear relationship between the number of laser shots and the decrease in $\mathrm{Sr}$ emission intensity illustrating that the greatest amounts of mass are removed during the first laser pulses.

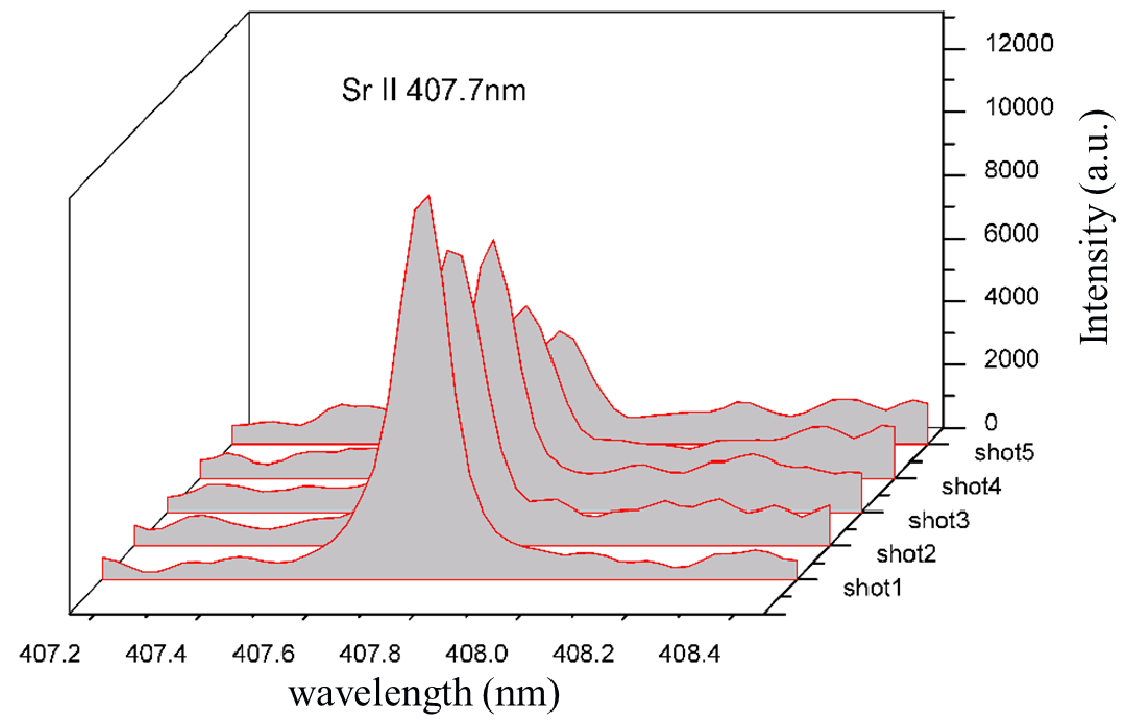

Figure 45. Linear relationship of Sr mass ablated per laser pulse

This experiment was conducted to conclude the number of shots needed to remove all the mass. After many trials were completed, investigating varying the number of drops with 
laser pulses, it was determined that 20 laser pulses was sufficient to remove all the deposited mass.

The 20 laser pulse accumulation acquired during LIBS analysis increases the crater depth from $\sim 15 \mu \mathrm{m}$, previously demonstrated in Figure 44 for microdrop deposition, to $\sim 165 \mu \mathrm{m}$ depth after LIBS ablation, see Figure 46.

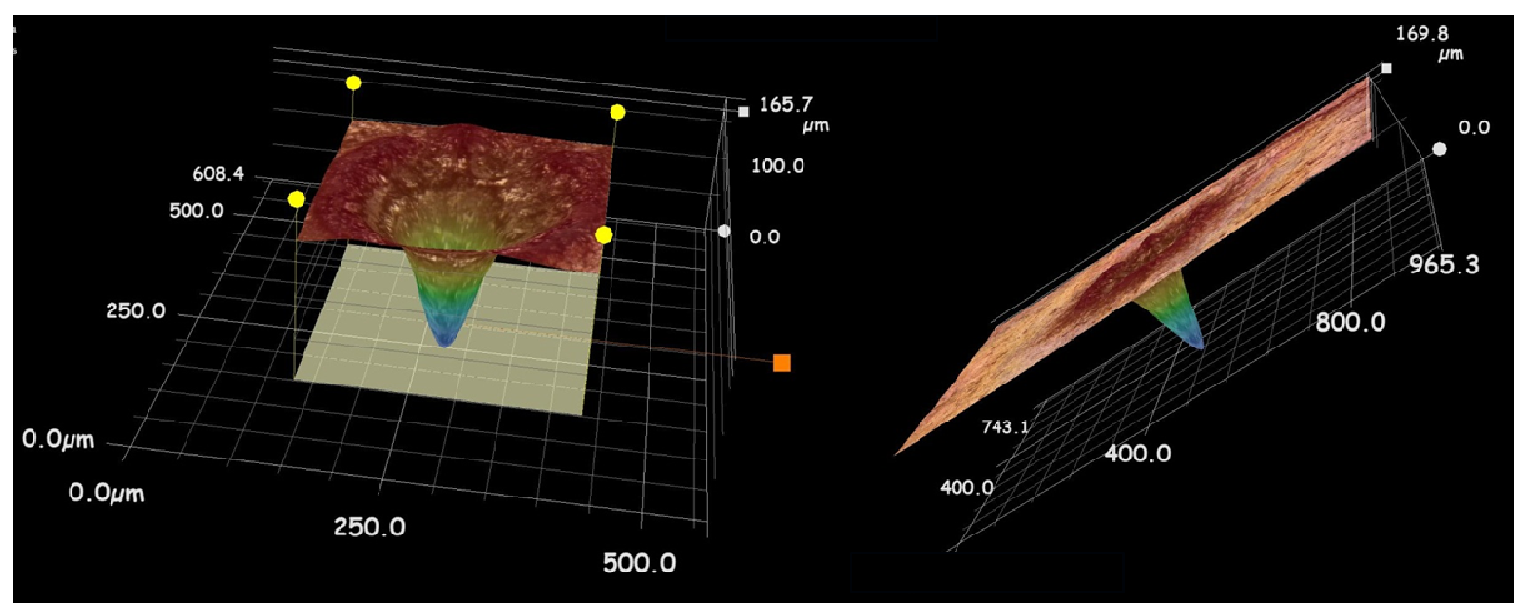

Figure 46. Increase in crater depth on Al stub after LIBS 20 laser pulse accumulation LIBS analysis As a result of ablating predominantly the aluminum stub for the microdrop analysis, the background is dominated by aluminum and other major elemental emission lines $(\mathrm{Fe}, \mathrm{Si}$ and $\mathrm{Cu})$. Focusing in on the analyte's region of interest, a narrow peak is illustrated, produced from the deposition of $150 \mathrm{pg}$, see Figure 47. 


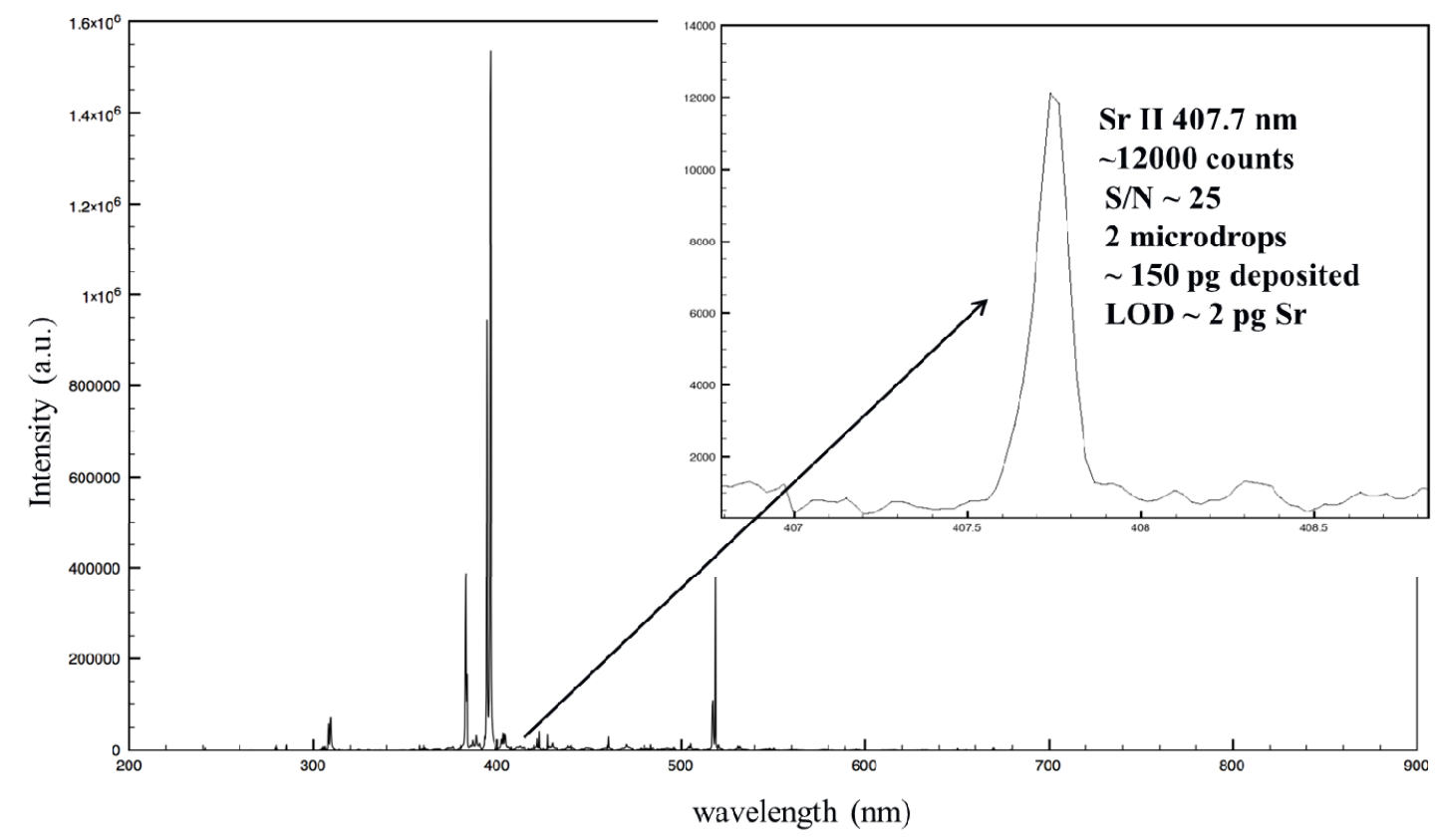

Figure 47. Al stub spectrum with $150 \mathrm{pg}$ of $\mathrm{Sr}$ deposited into crater

The spectrum in Figure 47 illustrates there is no other interference from the aluminum matrix or the solvent. The areas around the crater were also analyzed to verify no solution was spilled. The analysis of the area around the crater produced no signal for the analytes of interest.

Varying the number of drops printed in each crater varied the quantity of mass deposited. Calibration curves were produced correlating the absolute mass ablated to the LIBS background subtracted intensity. The number of microdrops printed in each crater varied from 0 to 12 drops of $250 \mathrm{ppm} \mathrm{Ba}$ and Sr solution. All points on the calibration curves, see Figure 48, represent an average of 5 replicates and the error bars represent \pm 1 standard deviation. 

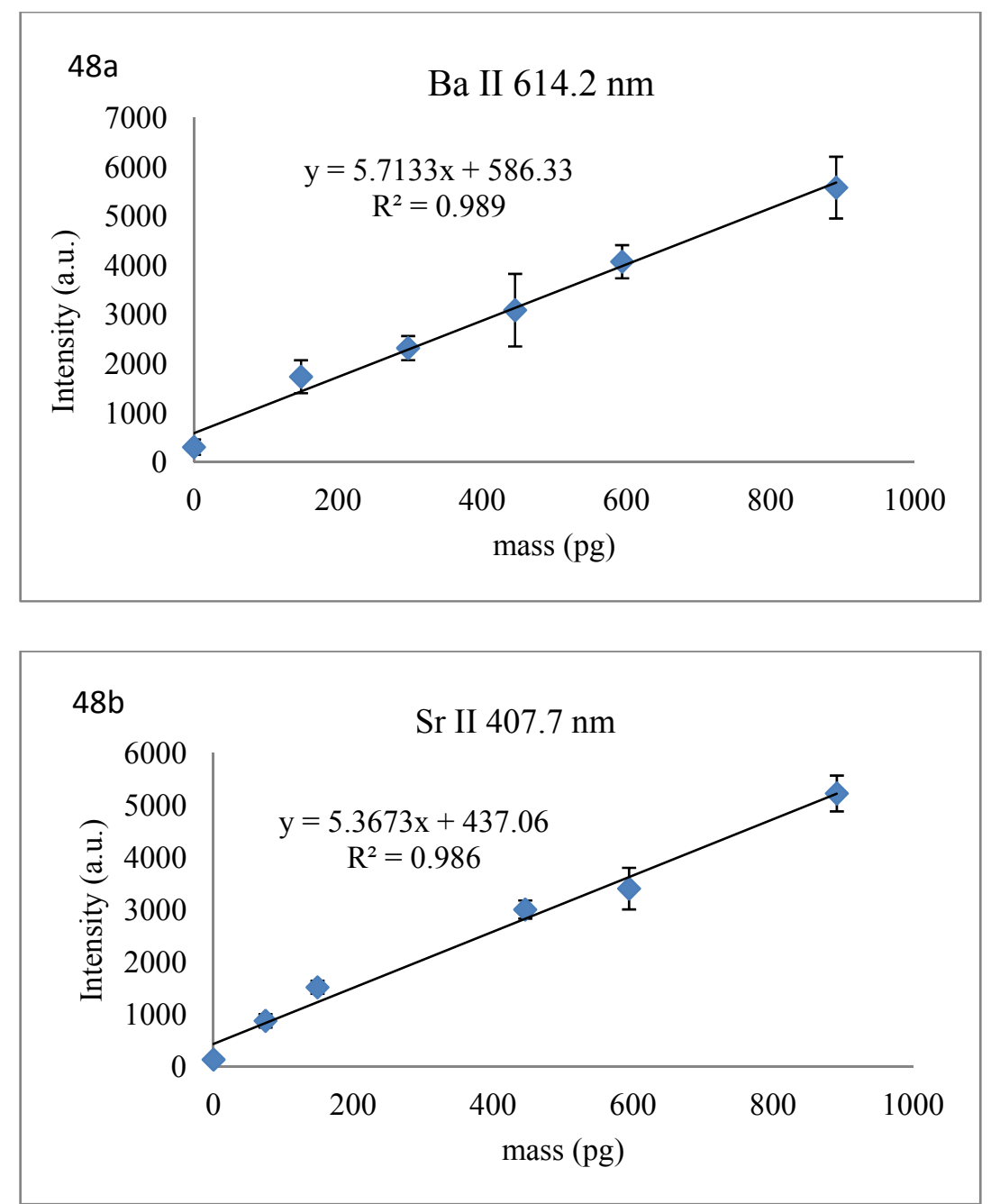

Figure 48. Calibration curves for microdrop LIBS on aluminum stub for a) Sr II $407.7 \mathrm{~nm}$ and b) Ba II $614.2 \mathrm{~nm}$

The precision for Ba II $614.2 \mathrm{~nm}$ ranged from $~ 12 \%$ RSD for 10 drops (215 pg) to $\sim 20 \%$ RSD for 1 drop (21 pg). The precision for Sr II $407.7 \mathrm{~nm}$ ranged from $\sim 5 \% \mathrm{RSD}$ for 6 drops (445 pg) to $\sim 14 \%$ RSD for 1 drop (74 pg).

Continuing calibration verification standards (CCV) containing 250 ppm Sr was analyzed to test the bias of this method. For 6 drops (445 pg) a bias was produced of $7.5 \%$ corresponding to a $17 \%$ RSD and 8 microdrops (594 pg) produced a $6.9 \%$ bias and a precision of $7 \% \mathrm{RSD}$ for $\mathrm{Sr}$. 
The limit of detection is defined as the smallest measure that can be detected with reasonable certainty for a given analytical procedure and equivalent to $3 \sigma+\bar{x}$ where $\sigma$ is the standard deviation from a range in the background close the signal and $\bar{x}$ is the mean of the background. The calculated LOD for Sr II $407.7 \mathrm{~nm}$ and Ba II $614.7 \mathrm{~nm}$ were $\sim 2$ pg.

Utilizing inkjet printing for the deposition of metal solutions on aluminum stubs proved to be a viable method for the determination for absolute mass detected with LIBS. The only drawback was the background was dominated by the matrix of the aluminum stub.

\subsubsection{Microdrop Mapping with LIBS}

LIBS provides rapid elemental analysis and with only removing nanograms to micrograms of mass. LIBS is suited to elementally characterize surfaces with little-to-no sample preparation. Surface mapping by LIBS was reported in the mid 1990's.

Hakkanen et al. reported good correlation between $\mathrm{Ca}$ and Si concentrations in paper $^{183}$. Laserna et al. investigated two dimensional mapping of carbon impurities and reported depth resolution of $40 \mathrm{~nm}^{184}$. Menut et al. used LIBS to map the minor constituents of the surface on steel ${ }^{185}$. However, Menut et al reported that there was insufficient LIBS signal for trace elements that were measured at the $1 \%$ abundance level.

The goal of this next study is to demonstrate that LIBS is a sensitive technique that is capable of characterizing nanogram metal depositions on an aluminum substrate. The Jetlab 4 was programmed by Dr. Joshi-Kumar to print a pattern, see Figure 49. 


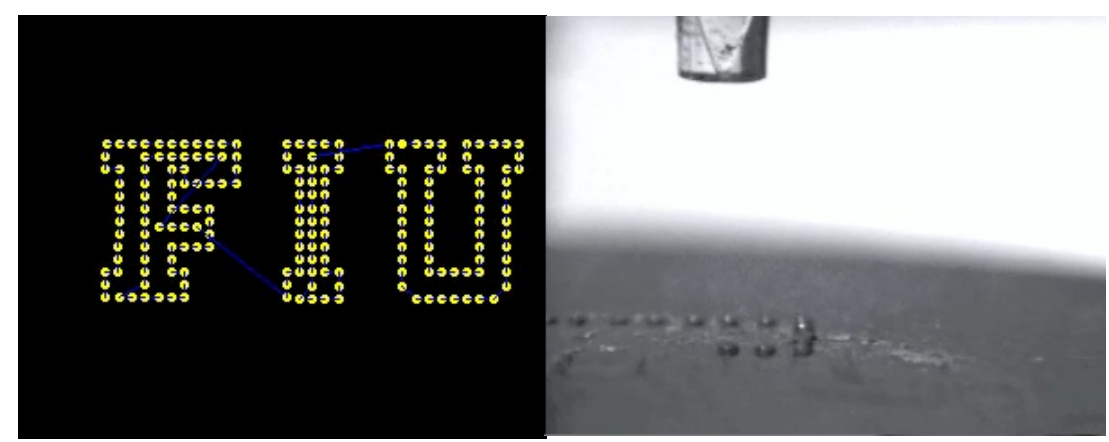

Figure 49. Jetlab 4 pattern printed for LIBS elemental mapping analysis

The software provided with the Jetlab 4 allows for a certain number of microdrops to be dropped at certain predetermined distances from each other. The software was programmed to print the FIU logo with microdrops being spaced $0.25 \mathrm{~mm}$.

Previously, premade craters were utilized for sample deposition and laser focus. This study printed one drop of 5000 ppm Sr solution, in the FIU pattern shown above, on an unaltered aluminum stub shown in Figure 50.

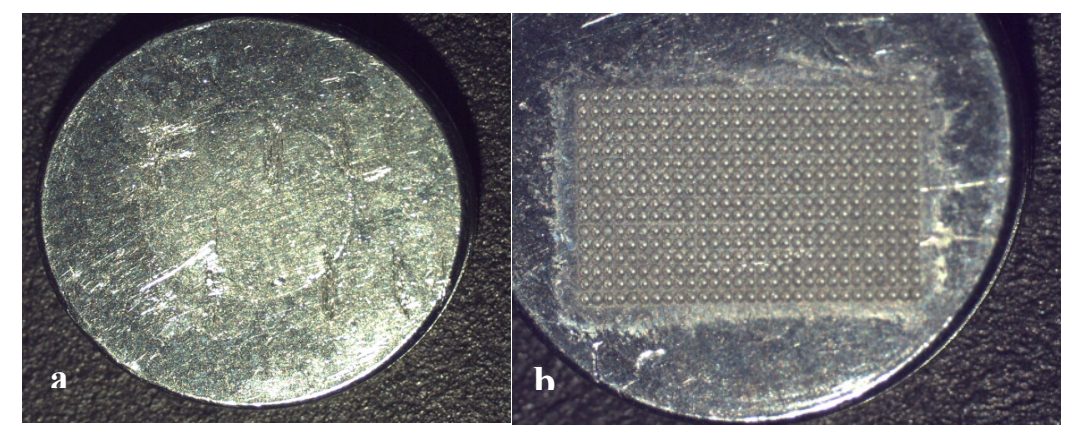

Figure 50. a) aluminum stub with printed pattern, b) aluminum stub after LIBS elemental mapping analysis

The resolution of the LIBS mapping was dependent on laser spot size and smallest distance the $x-y$ stage could translate. A $266 \mathrm{~nm}$ laser with a spot size of $215 \mu \mathrm{m}$ moved in steps of $200 \mu \mathrm{m}$ across the aluminum stub, see Figure 50b. The area elementally mapped by LIBS was $\sim 2.0 \mathrm{~cm}$ by $1.2 \mathrm{~cm}$, corresponding to approximately 50 laser craters by 30 laser craters. The line scan was stopped when analytical signal for Sr was blank for 
5 replicates. The data for the background subtracted peaks were entered into a Wolfram Mathematica ${ }^{\circledR}$ algorithm written by a previous post-doctoral student, Dr. Barnett, for image mapping. Figure 51 clearly illustrates the FIU logo in a two-dimensional plot.

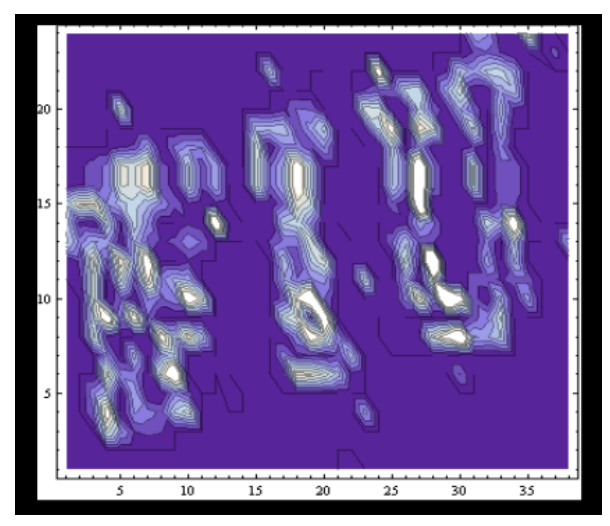

Figure 51. Two-dimensional plot of LIBS elemental mapping of $\mathrm{Sr}$

A three-dimensional elemental map was also constructed to illustrate the $\mathrm{Sr}$ concentration gradient across the aluminum stub, see Figure 52. The printed area relates to the presence of $\mathrm{Sr}$ concentration which was spatially mapped by signal intensity. The absolute mass detected for the elemental mapping of Sr was $26 \mathrm{ng}$ seen in the light regions of the plot.

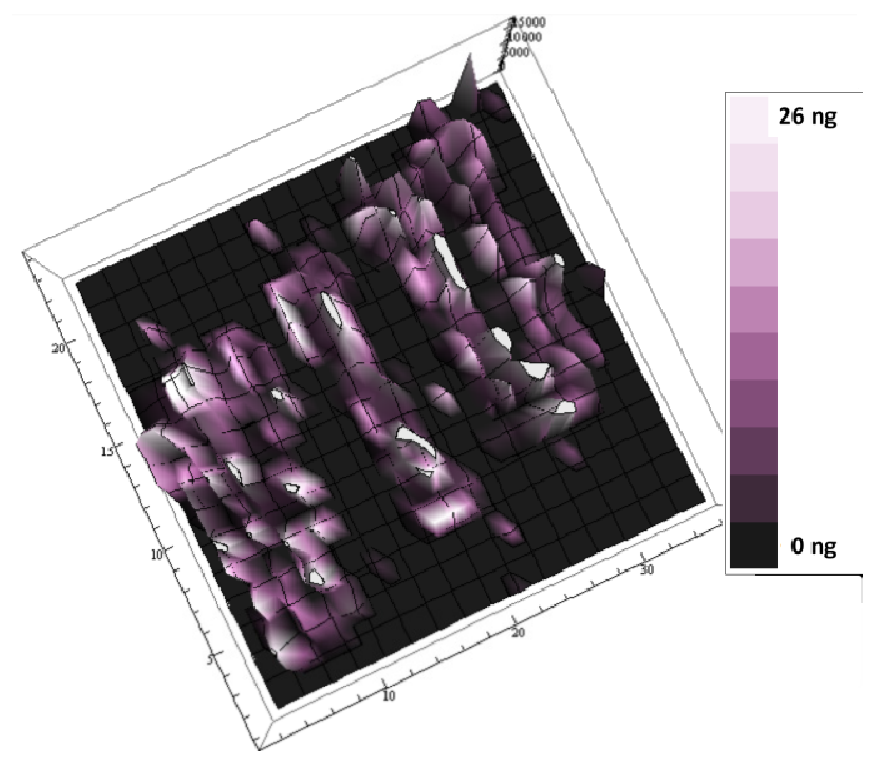

Figure 52. Three-dimensional plot illustrating Sr concentration with LIBS elemental mapping 
LIBS has demonstrated to be capable of providing special distribution of elements at very low mass loadings. LIBS could be an alternative method for the mapping of mineral, biological and forensic matrices.

\subsubsection{Microdrop Analysis with LIBS in Air}

As previously mentioned, the major disadvantage for microdrop LIBS analysis on the aluminum stub was the production of the high background produced as a result ablating predominantly the aluminum stub. In order to remove background inferences, the microdrop need to be analyzed without deposition on any surface.

For this study the JetDrive ${ }^{\mathrm{TM}} \mathrm{III}$ controller (Microfab Technologies, Inc., Plano, TX, USA) with a piezo-actuated $60 \mu \mathrm{m}$ orifice diameter printhead device was used to deliver microdrops of known mass quantities.

The microdrop printing parameters were optimized for drop size, velocity, and reproducibility. The driving waveform bipolar pulse amplitudes were $\pm 16 \mathrm{~V}$, a rise and fall time of $3 \mu$ s and a dwell time of $20 \mu$ s, with an ejection frequency of $250 \mathrm{~Hz}$. All experiments were performed under ambient temperature and pressure. These microdrops of known mass quantities were used to determine the LOD for absolute mass detection, bias and precision of LIBS.

A $532 \mathrm{~nm}$ laser was used for microdrop atomization and ionization. LIBS optical emissions were collected and imaged at a 90 degree angle from the plasma. The light was transferred into an AndorMechelle 5000 spectrometer. The experimental setup is seen in Figure 53 below. Five replicates were analyzed and averaged for the calculations. 


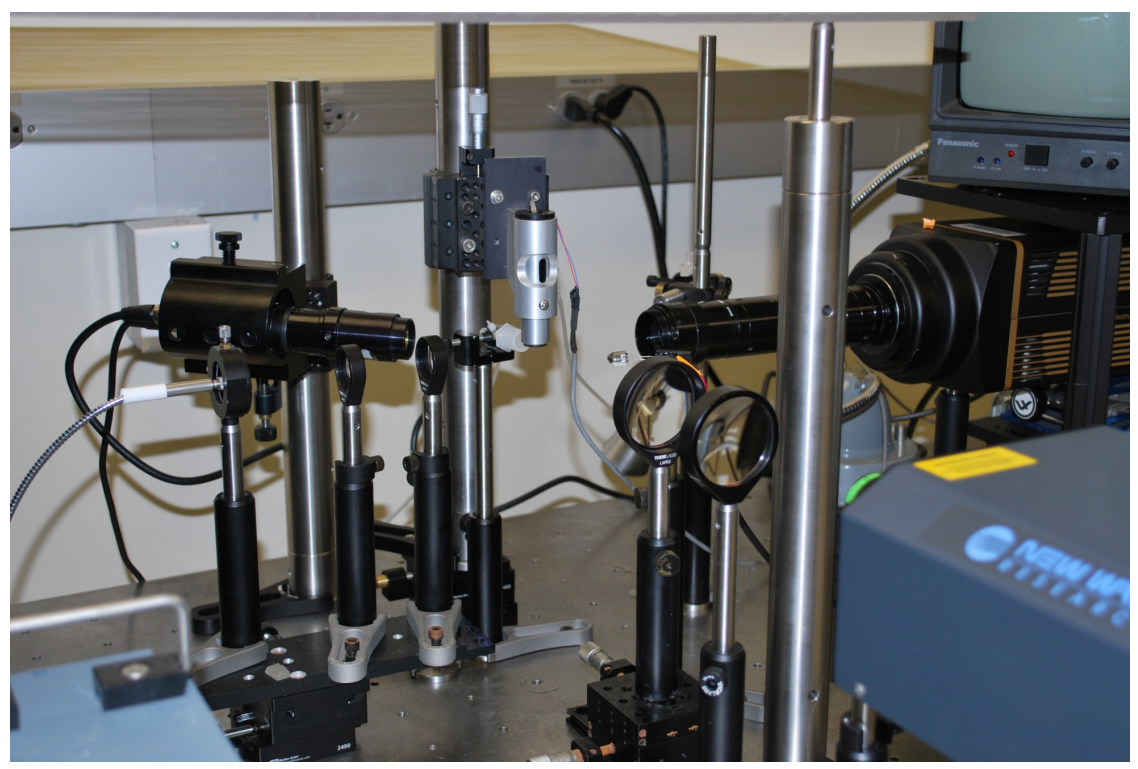

Figure 53. Experimental LIBS setup for microdrop LIBS in air

To prevent external influences from shifting the microdrop in flight, a Plexiglas shelter was constructed enclosing the collection optics and the microdrop printer. The printhead was placed approximately $4 \mathrm{~mm}$ above the formation of the LIBS plasma to reduce the time and length the drop needed to fall.

The drops were spatially and temporally aligned with the LIBS plasma using a PIMAX nanosecond gated camera. A strobed LED was used for observing droplets in flight to verify that only one drop per LIBS plasma was analyzed. The strobe provided a $3000 \mu$ s window, which allowed for visualization before the microdrop had formed, during microdrop formation and flight into the LIBS plasma.

The microdrop images were taken throughout the experiment to determine a drop volume of $90 \mathrm{pL} \pm 12 \mathrm{pL}$. An image of the printhead with a microdrop exiting and a microdrop in the LIBS plasma is shown in Figure 54. The drop size was determined by viewing images of the drop from a $90^{\circ}$ angle using simple graphing software to determine the number of pixels per square $\mathrm{mm}$ of the image. Using the formula for 
volume of a sphere, the drop volume was calculated. With the microdrop having a density of $1 \mathrm{~g} / \mathrm{cm}^{3}$, the terminal velocity is calculated to be $\sim 75 \mathrm{~mm} / \mathrm{sec}$.

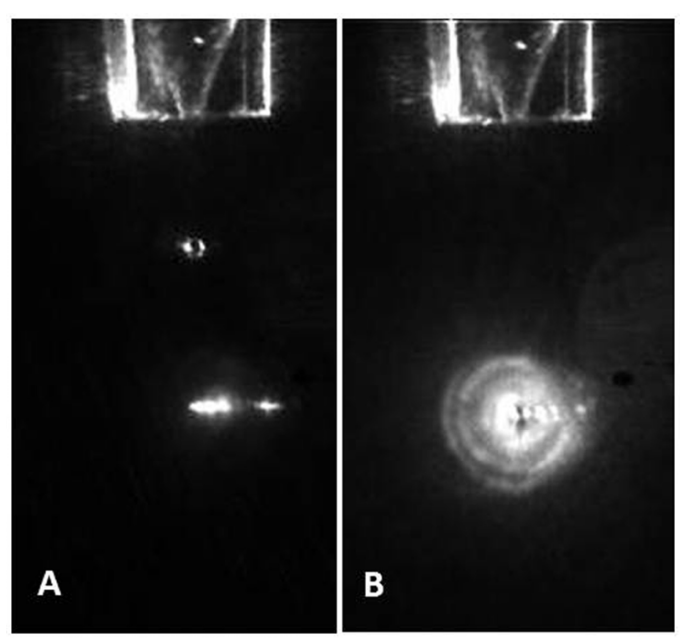

Figure 54. A) $90 \mathrm{pL}$ drop ejected from the orifice of the printhead; B) The microdrop in the LIBS plasma being atomized and ionized.

\subsubsection{Microdrop Data Analysis}

For single drop microdrop LIBS elemental solutions containing $\mathrm{Ba}, \mathrm{Sr}, \mathrm{Mg}$ and $\mathrm{Al}$ and were prepared to a final concentration of $0,10,15,25,50,100,250,500,1000$, and 3000 ppm thereby giving an absolute mass of $0,0.9,1.4,2.3,4.5,9.0,22.5,45.0,90.0$, and 270 pg per drop, respectively . Continuing calibration verification standards (CCVs) were prepared independently and used for method validation and to quantify bias at $25 \mathrm{ppm}$ for $250 \mathrm{ppm}(22.5 \mathrm{pg})$ for single drop microdrop LIBS. The CCVs were analyzed randomly throughout the experimental procedure.

As a result of continuous mode microdrop printing, more microdrops were produced than atomized and ionized by the LIBS plasma. To account for this, a kinetic series of 100 single laser pulses were performed and those with no signal were discarded, $\sim 30 \%$ of spectra were kept for analysis. Background subtracted peak intensities of the 
selected emission lines, determined to be free of spectral interferences were used for analysis

\subsubsection{Single-Pulse and Double-Pulse Microdrop LIBS}

Single-pulse and collinear double-pulse comparisons were conducted for microdrop LIBS at the optimized parameters listed above. As expected, an increase from single to doublepulse was observed. Figure 55 compares the LIBS spectra for single and double-pulse.

There is an approximate four-fold increase from single to double-pulse microdrop LIBS.

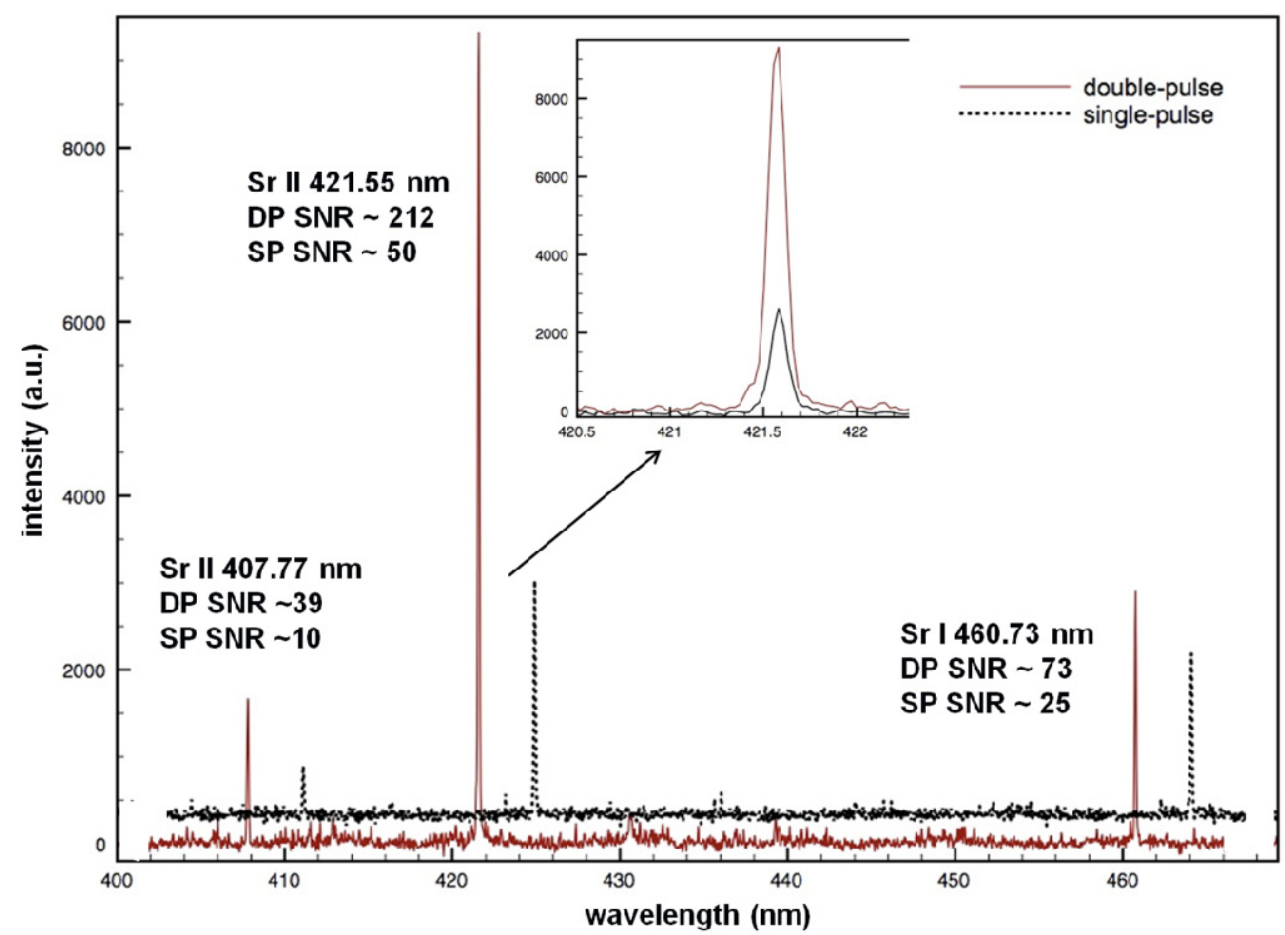

Figure 55. LIBS spectra of a microdrop double-pulse and single-pulse spectra for Sr peaks $407.7 \mathrm{~nm}$,

$$
421.5 \mathrm{~nm} \text { and } 460.7 \mathrm{~nm} \text { with } 90 \text { picogram of } \mathrm{Sr}
$$

The signal-to-noise ratio for the Sr II $407.7 \mathrm{~nm}$ emission line increased from 10 to 39 and Sr II $421.5 \mathrm{~nm}$ emission line signal-to-noise ratio increased from 50 to 212 . The signal 
enhancement for the atomic $\mathrm{Sr} 460.7 \mathrm{~nm}$ emission line was less at approximately 3 times with a single-pulse signal-to-noise of 25 and a collinear double-pulse of 73.

Single-drops produced volumes of $\sim 90 \mathrm{pL} \pm 12 \mathrm{pL}$ and directed to the space where a LIBS plasma would be formed. The varying concentrations of known mass where then jetted into the LIBS plasma and calibration curves were constructed. Weighted regression lines were used to treat the data for the microdrop LIBS as data heteroscedasticity was observed. Using weighted calibration curves allowed for additional weight to be added to the data points where the error was the smallest, thereby allowing for the best fit line to pass closer to those points with small error than to the points with larger error and concentration.38 The LIBS signal response to a known mass concentration was established producing $\mathrm{R}^{2}>0.99$ for both single and double-pulse configurations.

To assess the figures of merit for the single microdrop technique a CCV at a concentration of $250 \mathrm{ppm}$ was used, thereby producing a drop of containing $22.5 \mathrm{pg}$ of the analyte of interest. The precision of the microdrop LIBS was not as good as with aerosol LIBS with values ranging from $13 \%$ RSD, $16 \%$ RSD and 22\% RSD for Al II $394.4 \mathrm{~nm}$, Sr II $421.5 \mathrm{~nm}$ and Ba II $493.41 \mathrm{~nm}$, respectively. However, single microdrop LIBS was able to achieve a bias of 1\% and 5\% for emission lines Al II $394.4 \mathrm{~nm}$ and Ba II $493.4 \mathrm{~nm}$, and these results are summarized in Table 17. 
Table 19. Figures of Merit for Microdrop LIBS in Air

\begin{tabular}{|c|c|c|c|c|c|c|}
\hline \multicolumn{7}{|c|}{ Microdrop LIBS } \\
\hline $\begin{array}{c}\text { Emission } \\
\text { Line }\end{array}$ & Mass (pg) & $\begin{array}{c}\text { Volume } \\
\text { (pL) }\end{array}$ & SNR & $\begin{array}{c}\text { Bias } \\
\text { (\%) }\end{array}$ & $\begin{array}{c}\text { Precision } \\
(\%)\end{array}$ & $\begin{array}{c}\text { LOD } \\
\text { (pg) }\end{array}$ \\
\hline Sr II 421 & 45 & 90 & 69 & 2.6 & 8.3 & 1 \\
\hline Mg II 279 & 45 & 90 & 13 & 9.0 & 15.2 & 1 \\
\hline Ba II 493 & 45 & 90 & 34 & -5.1 & 22.1 & 1 \\
\hline Al II 394 & 45 & 90 & 15 & 1.1 & 13.2 & 3 \\
\hline
\end{tabular}

Microdrop LIBS resulted in LODs of approximately 1 pg for Ba II $493.4 \mathrm{~nm}$, Sr II $421.5 \mathrm{~nm}$ and Mg II $279.8 \mathrm{~nm}$. The Al II $394.4 \mathrm{~nm}$ emission line resulted in a LOD of approximately $3 \mathrm{pg}$. The larger LOD is the result of the incorporation of the entire drop volume (90 pL) for the observation. Other LIBS researchers such as, Feng et al., found the $\mathrm{Pb}$ I 405.7 emission line LOD to be $60 \mu \mathrm{g} / \mathrm{mL}$ when using a liquid jet at a flow rate of $20 \mathrm{ml} / \mathrm{min}^{54}$, Kumar et al. used liquid jets at different flows rates, $17.5 \mathrm{ml} / \mathrm{min}$ and 3.5 $\mathrm{ml} / \mathrm{min}$, and found $\mathrm{Mg}$ II $279.8 \mathrm{LOD}$ to be $0.06 \mu \mathrm{g} / \mathrm{mL}^{53}$ and Knoch et al. list a LOD of Mn at $80 \mu \mathrm{g} / \mathrm{L}$ when forming a cavitation bubble inside the cuvette ${ }^{56}$. Both methods described in this paper use magnitudes of volume less and produce LODs of $\sim 1 \mu \mathrm{g} / \mathrm{mL}$ (100 fg) for aerosol LIBS and $\sim 1$ pg for single drop microdrop LIBS. 


\subsection{Internal Standardization for Solution Analysis by LIBS}

Internal standardization was evaluated for improving sensitivity, precision and bias for both aerosol and microdrop LIBS. Previous research by $\mathrm{Ko}^{186}$ on solid matrices recommends two conditions should be met for an element to be used as an internal standard: the emission lines need to be close in the broadband spectrum and the excitation of the upper energy levels of the two transitions should be within the same range. During the study different elements and different elemental emission lines of the same element with varying excitation energies were evaluated. Fichet et al. previously reported the use of an internal standard as deteriorating the linearity and limit of detection ${ }^{187}$, however, Koch et al. find internal standardization to improve the signal ${ }^{56}$. During the present study scandium, sodium and copper were used as internal standards and added to have total concentrations in the solutions of 25,50 or $75 \mathrm{ppm}$. Off these three different concentrations in aerosol LIBS, 25 ppm scandium produced most favorably with a precision average of $3.4 \% \mathrm{RSD}$, a bias of $1 \%$ and a LOD of $0.8 \mathrm{ppm}$. Similar to worse results were obtained for different emission lines and different elements. None of the elements or corresponding emission lines chosen for internal standards elements exhibited a significant improvement for the figures of merit in either aerosol or microdrop LIBS. 


\subsection{Conclusions}

Aerosol LIBS and single drop microdrop LIBS allow for solution samples to be analyzed directly in air or without a carrier gas. These two techniques eliminate certain disadvantages observed with liquid analyses, such as splashing, while also eliminating the greater volume requirements needed for solution sampling by techniques such as ICPMS or ICP-OES.

When comparing the aerosol to the microdrop LIBS, the microdrop provides the mass in an area confined by the $\sim 90 \mathrm{pL}$ microdrop, where the mass ejected and detected in the aerosol is not as confined, thereby the amount of mass detected per unit area is less. Aerosol LIBS is dependent on the accumulation of 20 laser pulses to aid in achieving the low limits of detection. Microdrop LIBS did not use any accumulations, and only one laser pulse was necessary to integrate a $90 \mathrm{pL}$ microdrop.

Data analyzed in both the single and double-pulse configurations resulted in correlation coefficients $>0.99$ for both methods of aerosol LIBS and single drop microdrop LIBS. Microdrop LIBS on an aluminum stub produced correlation coefficients of $\leq 0.99$.

Quantitative analyses by aerosol LIBS demonstrated an ultra-low limit of detection of $\sim 100 \mathrm{fg}$ for an analytical volume of $20 \mu \mathrm{L}$ and produced a precision and accuracy values ranging from as low as $0.5 \% \mathrm{RSD}$ and $0.9 \%$, respectively in the collinear double-pulse LIBS configuration. Microdrop LIBS is demonstrated to have ultra-low absolute limits of detection of approximately $1 \mathrm{pg}$ in a total analytical volume of $90 \mathrm{pL}$ with an average precision of $14 \% \mathrm{RSD}$ and an average bias of $4.5 \%$. Microdrop LIBS on an aluminum stub performed similar to single microdrop LIBS producing a low 
absolute limit of detection of approximately $2 \mathrm{pg}$, with an average precision of $12 \% \mathrm{RSD}$ and an average bias of $8 \%$.

This research demonstrates that LIBS is able to accurately and precisely analyze small volumes of solution $\leq 300 \mu \mathrm{L}$ for aerosol analysis and $90 \mathrm{pL}$ for single-microdrops. These two methods dramatically reduce the total volume needed for analysis when compared to traditional solution sampling methods.

LIBS has shown the capability to accurately map surfaces by their elemental profile. LIBS detected absolute mass amounts less than or equal to $29 \mathrm{ng}$ on the sample surface. The elemental mapping by LIBS was time-consuming; however, elemental mapping done by SED and XRF can take hours also. The advantage to using LIBS is the low limit of detection. LIBS has the potential to provide surface analysis mapping, depth profiling and elemental profiling with high sensitivity. 


\subsection{Research Conclusions}

There have been many significant contributions to the LIBS literature since the first reporting of the use of a laser induced plasma as an excitation source in atomic spectroscopy in $1963^{75}$. Throughout this research, LIBS has demonstrated to be capable of both a qualitative and quantitative analysis for solutions and solids. One of the main advantage of LIBS is its simplicity. LIBS uses a high-power laser to produce a spark on the sample surface. This results in a high temperature plasma, where the emitted photons are collected from atoms and ions. The evolution of the transient plasma requires temporal gating to obtain the highest analytical signal. The collection of the emitted light allows for identification and quantification of the elements present in the sample. LIBS offers advantages over conventional elemental analysis techniques, in that it provides real time analysis on most all sample matrices without any sample preparation.

Precision is the key in forensic science as a result of comparisons being made between known and questioned samples. The forensic analyst needs to state with a high degree of certainty that the questioned sample is or is not significantly different than the known sample. These comparisons establish whether a sample has originated from a known source or that it is different and the observed differences are greater than the experimental error and sample heterogeneity, combined. LA-ICP-MS is a very sensitive analytical technique that most forensic laboratories in the United States do not have access to because the cost of acquisition and operation is out-of-reach. LIBS is not as sensitive as the competing technique of LA-ICP-MS, however, it has gained interest in that it is able to produce similar results at a fraction of the complexity and cost making it 
a very attractive analytical technique for the association and discrimination of glass fragments in the forensic laboratory.

The first study conducted was the optimum laser wavelength for the forensic analysis of glass using LIBS. It was proven that the UV laser provide better lasermaterial coupling and less plasma shielding. This leads to an increase mass amount ablated and an increase in analytical signal. The $266 \mathrm{~nm}$ wavelength provided a linear relationship to mass ablated achieving correlation coefficients of $>0.990$, a precision of $\sim 4.5 \% \mathrm{RSD}$ for elements ranging from $32-4600 \mathrm{ppm}$, and LOD where more than adequate to glass analysis. Where the $1064 \mathrm{~nm}$ laser still provided analytical data, it destroyed the sample and the precision increased to $\sim 10 \%$ RSD.

LIBS was used as an analytical technique for the forensic association and discrimination of glass produced at the same manufacturing plant using the developed method. Two different sample sets were used to assess the discrimination power of an optimized analytical LIBS method for the elemental analysis of glass. The precision of developed method for calculating the ratios was determined to be $\sim 5.5 \%$ RSD. The samples ranged in manufacturing date from days to years apart. When all the ratios are combined in a comparison, $99 \%$ of the possible pairs were discriminated using the optimized LIBS method. These results support the hypothesis that the elemental composition of glass produced in a single plant over time varies more than the differences that can be detected using analytical LIBS. The round robin studies were evidence that LIBS is able to produce similar results when the method is optimized.

LIBS demonstrates to be a powerful and sensitive technique for the forensic association and discrimination of float glass. LIBS has also shown to provide information 
of discrimination on other forensic matrices, such as, drugs ${ }^{188}$, explosives ${ }^{189,190}$, soil ${ }^{115}$, ink $^{191}, \operatorname{cotton}^{27}$ and paint ${ }^{192,193}$. LIBS instrumentation is currently being manufactured commercially, allowing LIBS to be easily implemented into forensic laboratories in the future. The portability of LIBS allows for instrumentation to be taken to the crime scene, thereby reducing possible contamination sources. LIBS is an attractive analytical method for forensic laboratories because of the low LODs achieved and the cost of acquisition, operation and maintenance being less than then the well-established techniques.

Finally LIBS was shown capable of low volume $(90 \mathrm{pL})$ quantitative elemental analysis of picogram amounts of dissolved metals in solutions. Single-pulse and collinear double-pulse LIBS were investigated using a $532 \mathrm{~nm}$ dual head laser.

The calibration curves produced correlation coefficients with $\mathrm{R}^{2}$ values $>0.99$ for both systems. The precision, accuracy and limit of detection (LOD) determined for aerosol LIBS were averaged and determined for the emission lines of Sr II $(421.5 \mathrm{~nm})$, Mg II (279.8 nm), Ba II (493.4 nm) and Ca II (396.8 nm) to be 3.8\% RSD, 3.1\% bias, $0.7 \mu \mathrm{g} / \mathrm{mL}$, respectively. A microdrop dispenser was used to deliver single drops onto an aluminum stub and into air. The microdrops allow for known mass amounts to be deposited onto a sample surface or captured in air.

In the single drop microdrop LIBS experiment, the analysis of a single drop, containing a total mass of $45 \mathrm{pg}$ resulted in a precision of $13 \%$ RSD and a bias of $1 \%$ for the Al I (394.4 nm) emission line. The absolute limits of detection for microdrop LIBS for the $\mathrm{Sr}$ II $421.5 \mathrm{~nm}$ emission line was approximately $1 \mathrm{pg}$ for both microdrops on an aluminum stub and in air. Overall, the precision, accuracy and absolute LOD determined 
for microdrop LIBS resulted in a typical performance of $\sim 14 \% \mathrm{RSD}, 6 \%$ bias, and $1 \mathrm{pg}$ for the elements Sr II (421.5 nm), Al I(394.4 nm), Mg II (279.8) and Ba II (493.4 nm).

Aerosol LIBS used $\sim 20 \mu \mathrm{L}$ per analysis and was able to produce bias of as low as $0.9 \%$ for $\mathrm{Sr}$ II $421.5 \mathrm{~nm}$ the emission line. The LOD of approximately $120 \mathrm{fg}$ was achieved for Sr II $421.5 \mathrm{~nm}, 170 \mathrm{fg}$ for Ba II $493.4 \mathrm{~nm}, 70 \mathrm{fg}$ for Ca II $396.3 \mathrm{~nm}$ and reached as low as $30 \mathrm{fg}$ for $\mathrm{Mg}$ II $279.80 \mathrm{~nm}$.

The real-time analyses of microdrops, aerosols and particles have a wide range of applications. The future of this analytical method is predicted to be implemented for analysis of combustion process monitoring, pollutants of heavy metals in air, waste monitoring into water streams, and atmospheric sciences. The LIBS instrumentation can be manufactured compact and portable which allows for in-situ monitoring of these many applications.

The development and implementation of technical standards is important for the future growth of LIBS as an analytical technique. Standard operating procedures and standard methodology needs to be developed, similar to LA-ICP-MS. The results presented throughout this research demonstrate that LIBS is capable for the analysis of glass as it provides similar discrimination as LA-ICP-MS. LIBS also was proven capable for the analysis of small volumes, thereby competing with the solution technique of ICPOES. 


\section{REFERENCE LIST}

1. Buscaglia, J.; Koons, R. D. Distribution of Refractive Index Values in Sheet Glasses. Forensic Science Communications 2001, 3(1).

2. Buscaglia, J. A. Elemental Analysis of Small Glass Fragments in ForensicScience. Analytica Chimica Acta 1994, 288 (1-2), 17-24.

3. Naes, B. E.; Umpierrez, S.; Ryland, S.; Barnett, C.; Almirall, J. R. A comparison of laser ablation inductively coupled plasma mass spectrometry, micro X-ray fluorescence spectroscopy, and laser induced breakdown spectroscopy for the discrimination of automotive glass. Spectrochimica Acta Part B-Atomic Spectroscopy 2008, 63 (10), 1145-1150.

4. Zadora, G. Glass analysis for forensic purposes - a comparison of classification methods. Journal of Chemometrics 2007, 21 (5-6), 174-186.

5. Almirall, J.; Trejos, T. Advances in the forensic analysis of glass fragments with a focus on refractive index and elemental analysis. Forensic Sci. Rev. 2006, 2 (18), 73-76.

6. Khorrami, A. R.; Fakhari, A. R.; Shamsipur, M.; Naeimi, H. Pre-concentration of ultra trace amounts of copper, zinc, cobalt and nickel in environmental water samples using modified C18 extraction disks and determination by inductively coupled plasma-optical emission spectrometry. International Journal of Environmental Analytical Chemistry 2009, 89 (5), 319-329.

7. Duckworth, D. C.; Morton, S. J.; Bayne, C. K.; Koons, R. D.; Montero, S.; Almirall, J. R. Forensic glass analysis by ICP-MS: a multi-element assessment of discriminating power via analysis of variance and pairwise comparisons. J. Anal. Atom. Spectrom. 2002, 17 (7), 662-668.

8. Coleman, R. F.; Goode, G. C. Comparison of glass fragments by neutron activation analysis. J. Radioanal. Chem. 1973, 15, 367-388.

9. Koons, R. D.; Fiedler, C.; Rawalt, R. C. Classification and Discrimination of Sheet and Container Glasses by Inductively Coupled Plasma-Atomic EmissionSpectrometry and Pattern-Recognition. J. Forensic Sci. 1988, 33 (1), 49-67.

10. Suzuki, Y.; Sugita, R.; Suzuki, S.; Marumo, Y. Determination of trace impurities in windshield glass by ICP-MS. Bunseki Kagaku 1997, 46 (10), 825-830.

11. Zurhaar, A.; Mullings, L. Characterization of Forensic Glass Samples Using Inductively Coupled Plasma Mass-Spectrometry. Journal of Analytical Atomic Spectrometry 1990, 5 (7), 611-617. 
12. Berends-Montero, S.; Wiarda, W.; de Joode, P.; van der Peijl, G. Forensic analysis of float glass using laser ablation inductively coupled plasma mass spectrometry (LA-ICP-MS): validation of a method. J. Anal. Atom. Spectrom. 2006, 21 (11), 1185-1193.

13. Bridge, C. M.; Powell, J.; Steele, K. L.; Williams, M.; MacInnis, J. M.; Sigman, M. E. Characterization of automobile float glass with laser-induced breakdown spectroscopy and laser ablation inductively coupled plasma mass spectrometry. Appl. Spectrosc. 2006, 60 (10), 1181-1187.

14. Latkoczy, C.; Becker, S.; Ducking, M.; Gunther, D.; Hoogewerff, J. A.; Almirall, J. R.; Buscaglia, J.; Dobney, A.; Koons, R. D.; Montero, S.; van der Peijl, G. J. Q.; Stoecklein, W. R. S.; Trejos, T.; Watling, J. R.; Zdanowicz, V. S. Development and evaluation of a standard method for the quantitative determination of elements in float glass samples by LA-ICP-MS. Journal of Forensic Sciences 2005, 50 (6), 1327-1341.

15. Smith, K.; Trejos, T.; Watling, R. J.; Almirall, J. A guide for the quantitative elemental analysis of glass using laser ablation inductively coupled plasma mass spectrometry. Atom. Spectrosc. 2006, 27 (3), 69-75.

16. ASTM Standard Terminology of glass and glass products. Annual Book of ASTM Standards 2005, ASTM C, 162.

17. F.T.Maloney Glass in the Modern World; Aldus Books Ltd: 1968.

18. B.H.W.S.de Jong Ullmann's Encyclopedia of Industrial Chemistry. 7th Edition ed.; Wiley-VCH: 2011.

19. Hubert Schroeder Schott Guide to Glass; Van Nostrand Reinhold Company Inc.: 1983.

20. NSG Group Pilkington and the Flat Glass Industry 2010; 10.

21. Glass Association of North America . Glass in Today's Architecture. 2006.

22. B.German; D.Morgans; A.Butterworth; A.Scaplehorn A Survey of British Container Glass Using Spark Source Mass Spectrometry with Electrical Detection. J. Forensic Sci. 1978, 18, 113.

23. Koons, R. D.; Peters, C. A.; Rebbert, P. S. Comparison of Refractive-Index, Energy Dispersive-X-Ray Fluorescence and Inductively Coupled Plasma Atomic Emission-Spectrometry for Forensic Characterization of Sheet Glass Fragments. J. Anal. Atom. Spectrom. 1991, 6 (6), 451-456. 
24. J.Andrasko; A.C.Maehly The discrimination between samples of window glass by combining physical and chemical techniques. J. Forensic Sci. 1978, 23, 250.

25. Kuisma-Kursula, P. Accuracy, precision and detection limits of SEM-WDS, SEM-EDS and PIXE in the multi-elemental analysis of medieval glass. X-Ray Spectrometry 2000, 29 (1), 111-118.

26. Ryland, S. G. Sheet Or Container - Forensic Glass Comparisons with An Emphasis on Source Classification. J. Forensic Sci. 1986, 31 (4), 1314-1329.

27. Schenk, E. R.; Almirall, J. R. Elemental analysis of glass by laser ablation inductively coupled plasma optical emission spectrometry (LA-ICP-OES). Forensic Sci. Int. (0).

28. Rodriguez-Celis, E. M.; Gornushkin, I. B.; Heitmann, U. M.; Almirall, J. R.; Smith, B. W.; Winefordner, J. D.; Omenetto, N. Laser induced breakdown spectroscopy as a tool for discrimination of glass for forensic applications. Analytical and Bioanalytical Chemistry 2008, 391 (5), 1961-1968.

29. McIntee, E.; Viglino, E.; Kumor, S.; Rinke, C.; Ni, L. Q.; Sigman, M. E. Nonparametric permutation test for the discrimination of float glass samples based on LIBS spectra. Journal of Chemometrics 2010, 24 (5-6), 312-319.

30. Barnett, C.; Cahoon, E.; Almirall, J. R. Wavelength dependence on the elemental analysis of glass by Laser Induced Breakdown Spectroscopy. Spectrochimica Acta Part B-Atomic Spectroscopy 2008, 63 (10), 1016-1023.

31. Cahoon, E. M.; Almirall, J. R. Wavelength dependence on the forensic analysis of glass by nanosecond $266 \mathrm{~nm}$ and $1064 \mathrm{~nm}$ laser induced breakdown spectroscopy. Applied Optics 2010, 49 (13), C49-C57.

32. ASTM Standard test method for determination of trace elements in glass samples using inductively coupled plasma mass spectrometry (ICP-MS). E2330-04 2004.

33. Hickman, D. A. A Classification Scheme for Glass. Forensic Sci. Int. 1981, 17 (3), 265-281.

34. Hickman, D. A.; Harbottle, G.; Sayre, E. V. The Selection of the Best Elemental Variables for the Classification of Glass Samples. Forensic Sci. Int. 1983, 23 (23), 189-212.

35. Hickman, D. A. Elemental Analysis and the Discrimination of Sheet Glass Samples. Forensic Sci. Int. 1983, 23 (2-3), 213-223.

36. Hickman, D. A. Glass types identified by chemical analysis. Forensic Sci. Int. 1987, 33 (1), 23-46. 
37. Parouchais, T.; Warner, I. M.; Palmer, L. T.; Kobus, H. The analysis of small glass fragments using inductively coupled plasma mass spectrometry. J. Forensic Sci. 1996, 41 (3), 351-360.

38. Suzuki, Y.; Kasamatsu, M.; Sugita, R.; Ohta, H.; Suzuki, S.; Marumo, Y. Forensic discrimination of headlight glass by analysis of trace impurities with synchrotron radiation X-ray fluorescence spectrometry and ICP-MS. Bunseki Kagaku 2003, 52 (6), 469-474.

39. Gray, A. L. Solid Sample Introduction by Laser Ablation for Inductively Coupled Plasma Source-Mass Spectrometry. Analyst 1985, 110 (5), 551-556.

40. Trejos, T.; Montero, S.; Almirall, J. R. Analysis and comparison of glass fragments by laser ablation inductively coupled plasma mass spectrometry (LAICP-MS) and ICP-MS. Analytical and Bioanalytical Chemistry 2003, 376 (8), 1255-1264.

41. Trejos, T.; Almirall, J. R. Sampling strategies for the analysis of glass fragments by LA-ICP-MS - Part I. Micro-homogeneity study of glass and its application to the interpretation of forensic evidence. Talanta 2005, 67 (2), 388-395.

42. Bridge, C. M.; Powell, J.; Steele, K. L.; Sigman, M. E. Forensic comparative glass analysis by laser-induced breakdown spectroscopy. Spectrochimica Acta Part BAtomic Spectroscopy 2007, 62 (12), 1419-1425.

43. Naes, B. E.; Umpierrez, S.; Ryland, S.; Barnett, C.; Almirall, J. R. A comparison of laser ablation inductively coupled plasma mass spectrometry, micro X-ray fluorescence spectroscopy, and laser induced breakdown spectroscopy for the discrimination of automotive glass. Spectrochimica Acta Part B-Atomic Spectroscopy 2008, 63 (10), 1145-1150.

44. Schenk, E. R.; Almirall, J. R. Elemental analysis of cotton by laser-induced breakdown spectroscopy. Applied Optics 2010, 49 (13), 153-1.

45. Jeong, S. H.; Borisov, O. V.; Yoo, J. H.; Mao, X. L.; Russo, R. E. Effects of particle size distribution on inductively coupled plasma mass spectrometry signal intensity during laser ablation of glass samples. Analytical Chemistry 1999, 71 (22), 5123-5130.

46. Kuhn, H. R.; Gunther, D. Laser ablation-ICP-MS: particle size dependent elemental composition studies on filter-collected and online measured aerosols from glass. Journal of Analytical Atomic Spectrometry 2004, 19 (9), 1158-1164. 
47. Arroyo, L.; Trejos, T.; Gardinali, P. R.; Almirall, J. R. Optimization and validation of a Laser Ablation Inductively Coupled Plasma Mass Spectrometry method for the routine analysis of soils and sediments. Spectrochimica Acta Part B-Atomic Spectroscopy 2009, 64 (1), 16-25.

48. Duckworth, D. C.; Morton, S. J.; Bayne, C. K.; Koons, R. D.; Montero, S.; Almirall, J. R. Forensic glass analysis by ICP-MS: a multi-element assessment of discriminating power via analysis of variance and pairwise comparisons. Journal of Analytical Atomic Spectrometry 2002, 17 (7), 662-668.

49. Khorrami, A. R.; Fakhari, A. R.; Shamsipur, M.; Naeimi, H. Pre-concentration of ultra trace amounts of copper, zinc, cobalt and nickel in environmental water samples using modified C18 extraction disks and determination by inductively coupled plasma-optical emission spectrometry. International Journal of Environmental Analytical Chemistry 2009, 89 (5), 319-329.

50. Nyga, R.; Neu, W. Double-Pulse Technique for Optical-Emission Spectroscopy of Ablation Plasmas of Samples in Liquids. Optics Letters 1993, 18 (9), 747-749.

51. Oh, S. Y.; Miller, T.; Yueh, F. Y.; Singh, J. P. Comparative study of laser-induced breakdown spectroscopy measurement using two slurry circulation systems. Applied Optics 2007, 46 (19), 4020-4025.

52. Haisch, C.; Liermann, J.; Panne, U.; Niessner, R. Characterization of colloidal particles by laser-induced plasma spectroscopy (LIPS). Analytica Chimica Acta 1997, 346 (1), 23-35.

53. Kumar, A.; Yueh, F. Y.; Singh, J. P. Double-pulse laser-induced breakdown spectroscopy with liquid jets of different thicknesses. Applied Optics 2003, 42 (30), 6047-6051.

54. Feng, Y.; Yang, J. J.; Fan, J. M.; Yao, G. X.; Ji, X. H.; Zhang, X. Y.; Zheng, X. F.; Cui, Z. F. Investigation of laser-induced breakdown spectroscopy of a liquid jet. Applied Optics 2010, 49 (13), C70-C74.

55. Caceres, J. O.; Lopez, J. T.; Telle, H. H.; Urena, A. G. Quantitative analysis of trace metal ions in ice using laser-induced breakdown spectroscopy. Spectrochimica Acta Part B-Atomic Spectroscopy 2001, 56 (6), 831-838.

56. Koch, S.; Court, R.; Garen, W.; Neu, W.; Reuter, R. Detection of manganese in solution in cavitation bubbles using laser induced breakdown spectroscopy. Spectrochimica Acta Part B-Atomic Spectroscopy 2005, 60 (7-8), 1230-1235.

57. Cremers, D. A.; Radziemski, L. J.; Loree, T. R. Spectrochemical Analysis of Liquids Using the Laser Spark. Applied Spectroscopy 1984, 38 (5), 721-729. 
58. Pace, D. M. D.; D'Angelo, C. A.; Bertuccelli, D.; Bertuccelli, G. Analysis of heavy metals in liquids using Laser Induced Breakdown Spectroscopy by liquidto-solid matrix conversion. Spectrochimica Acta Part B-Atomic Spectroscopy 2006, 61 (8), 929-933.

59. S.Groh; P.K.Diwakar; C.C.Garcia; A.Murtazin; D.W.Hahn; K.Niemax 100\% Efficient Sub-Nanoliter Sample Introduction in Laser-Induced Breakdown Spectroscopy and Inductively Coupled Plasma Spectrometry: Implications of Ultralow Sample Volumes. Analytcal Chemistry 2010, 82 (6), 2568-2573.

60. P.K.Diwakar; S.Groh; K.Niemax; D.W.Hahn Study of analyte dissociation and diffusion in laser-induced plasmas: implications for laser-induced breakdown spectroscopy. J. Anal. At. Spectrom. 2010, 25, 1921-1930.

61. Janzen, C.; Fleige, R. +.; Noll, R.; Schwenke, H.; Lahmann, W.; Knoth, J.; Beaven, P.; Jantzen, E.; Oest, A.; Koke, P. Analysis of small droplets with a new detector for liquid chromatography based on laser-induced breakdown spectroscopy. Spectrochimica Acta Part B: Atomic Spectroscopy 2005, 60 (7-8), 993-1001.

62. Lees, E. R. Microdrop Generation; CRC Press: 2003.

63. Corsi, M.; Cristoforetti, G.; Giuffrida, M.; Hidalgo, M.; Legnaioli, S.; Palleschi, V.; Salvetti, A.; Tognoni, E.; Vallebona, C. Three-dimensional analysis of laser induced plasmas in single and double pulse configuration. Spectrochimica Acta Part B-Atomic Spectroscopy 2004, 59 (5), 723-735.

64. Noll, R.; Sattmann, R.; Sturm, V.; Winkelmarm, S. Space- and time-resolved dynamics of plasmas generated by laser double pulses interacting with metallic samples. Journal of Analytical Atomic Spectrometry 2004, 19 (4), 419-428.

65. Russo, R. E.; Mao, X. L.; Liu, C.; Gonzalez, J. Laser assisted plasma spectrochemistry: laser ablation. J. Anal. Atom. Spectrom. 2004, 19 (9), 10841089 .

66. Russo, R. E.; Suen, T. W.; Bol'shakov, A. A.; Yoo, J.; Sorkhabi, O.; Mao, X. L.; Gonzalez, J.; Oropeza, D.; Zorba, V. Laser plasma spectrochemistry. Journal of Analytical Atomic Spectrometry 2011, 26 (8), 1596-1603.

67. J.P.Singh; S.N.Thakur Laser-Induced Breakdown Spectroscopy; 1 ed.; Elsevier Science: 2007.

68. Russo, R. E.; Mao, X. L.; Mao, S. S. The physics of laser ablation in microchemical analysis. Analytical Chemistry 2002, 74 (3), 70A-77A.

69. F.F.Chen Introduction to Plasma Physics; Plenum: New York: 1974. 
70. J.P.Singh; S.N.Thakur Laser-Induced Breakdown Spectroscopy; First Edition ed.; Elsevier: 2007.

71. Mao, S. S.; Mao, X. L.; Greif, R.; Russo, R. E. Dynamics of an air breakdown plasma on a solid surface during picosecond laser ablation. Applied Physics Letters 2000, 76 (1), 31-33.

72. A.W.Miziolek; A.Palleschi; I.Schechter Laser-Induced Breakdown Spectroscopy Fundamentals and Applications; Cambridge Press: 2006.

73. Radziemski, L. J. From LASER to LIBS, the path of technology development. Spectrochimica Acta Part B-Atomic Spectroscopy 2002, 57 (7), 1109-1113.

74. F.Brech; L.Cross Optical microemission stimulated by a ruby laser. Spectroscopy $1962,16,59$.

75. J.Debras-Guédon; N.Liodec De l'utilisation du faisceau d'un amplificateur a ondes lumineuses par émission induite de rayonnement (laser á rubis), comme source énergetique pour l'excitation des spectres d'émission des éléments. C. R. Acad. Sci. 1963, 257, 3336.

76. H.Moenke; L.Moenke-Blankenburg Laser MicroSpectrochemical Analysis. Crane, Russak \& Co. New York: 1973.

77. W.W.Schroeder, J. J. v. N.; L.Dicks; A.Strasheim; H.V.D.Piepen A new eletronic time resolution system for direct reading spectrometers and some applications in the diagnosis of spark and laser radiations. Spectrochimica Acta Part B-Atomic Spectroscopy 1970, 26, 331-340.

78. Radziemski, L. J.; Loree, T. R. Laser-induced breakdown spectroscopy: Timeresolved spectrochemical applications. Plasma Chemistry and Plasma Processing 1981, $l$ (3), 281-293.

79. Radziemski, L. J.; Cremers, D. A.; Loree, T. R. Detection of Beryllium by LaserInduced-Breakdown Spectroscopy. Spectrochimica Acta Part B-Atomic Spectroscopy 1983, 38 (1-2), 349-355.

80. Cremers, D. A.; Radziemski, L. J. Direct Detection of Beryllium on Filters Using the Laser Spark. Appl. Spectrosc. 1985, 39 (1), 57-63.

81. David A.Cremers; Leon J.Radziemski Handbook of Laser-Induced Breakdown Spectroscopy; John Wiley \& Sons Ltd: 2006.

82. Cremers, D. A.; Radziemski, L. J.; Loree, T. R. Spectrochemical Analysis of Liquids Using the Laser Spark. Appl. Spectrosc. 1984, 38 (5), 721-729. 
83. Poulain, D. E.; Alexander, D. R. Influences on Concentration Measurements of Liquid Aerosols by Laser-Induced Breakdown Spectroscopy. Appl. Spectrosc. 1995, 49 (5), 569-579.

84. Aguilera, J. A.; Aragon, C.; Campos, J. Determination of Carbon Content in Steel Using Laser-Induced Breakdown Spectroscopy. Applied Spectroscopy 1992, 46 (9), 1382-1387.

85. Buckley, S. G.; Johnsen, H. A.; Hencken, K. R.; Hahn, D. W. Implementation of laser-induced breakdown spectroscopy as a continuous emissions monitor for toxic metals. Waste Management 2000, 20 (5-6), 455-462.

86. Palanco, S.; Laserna, J. J. Full automation of a laser-induced breakdown spectrometer for quality assessment in the steel industry with sample handling, surface preparation and quantitative analysis capabilities. J. Anal. Atom. Spectrom. 2000, 15 (10), 1321-1327.

87. Knight, A. K.; Scherbarth, N. L.; Cremers, D. A.; Ferris, M. J. Characterization of laser-induced breakdown spectroscopy (LIBS) for application to space exploration. Appl. Spectrosc. 2000, 54 (3), 331-340.

88. T.P.Hughes Plasmas and Laser Light; John Wiley: New York: 1975.

89. Liu, H. C.; Mao, X. L.; Yoo, J. H.; Russo, R. E. Early phase laser induced plasma diagnostics and mass removal during single-pulse laser ablation of silicon. Spectrochimica Acta Part B-Atomic Spectroscopy 1999, 54 (11), 1607-1624.

90. H.R.Griem Principles of Plasma Spectroscopy; Cambridge: 1997.

91. Zeng, X. H.; Mao, S. S.; Liu, C. Y.; Mao, X. L.; Greif, R.; Russo, R. E. Plasma diagnostics during laser ablation in a cavity. Spectrochimica Acta Part B-Atomic Spectroscopy 2003, 58 (5), 867-877.

92. Acquaviva, S.; D'Anna, E.; De Giorgi, M. L.; Moro, F. Laser-induced breakdown spectroscopy for compositional analysis of multielemental thin films. Spectrochimica Acta Part B-Atomic Spectroscopy 2006, 61 (7), 810-816.

93. Corsi, M.; Cristoforetti, G.; Giuffrida, M.; Hidalgo, M.; Legnaioli, S.; Palleschi, V.; Salvetti, A.; Tognoni, E.; Vallebona, C. Three-dimensional analysis of laser induced plasmas in single and double pulse configuration. Spectrochimica Acta Part B-Atomic Spectroscopy 2004, 59 (5), 723-735.

94. Yalcin, S.; Crosley, D. R.; Smith, G. P.; Faris, G. W. Influence of ambient conditions on the laser air spark. Applied Physics B-Lasers and Optics 1999, 68 (1), 121-130. 
95. Aguilera, J. A.; Aragon, C. Multi-element Saha-Boltzmann and Boltzmann plots in laser-induced plasmas. Spectrochimica Acta Part B-Atomic Spectroscopy 2007, 62 (4), 378-385.

96. C.Fleurier; S.Sahal-Brechot; J.Chapelle . Stark profiles of Al I and A1 II lines. J.Phys.B: Atom.Molec.Phys 10, 3435-3441. 1977.

97. Cristoforetti, G.; Legnaioli, S.; Palleschi, V.; Salvetti, A.; Tognoni, E. Effect of target composition on the emission enhancement observed in Double-Pulse LaserInduced Breakdown Spectroscopy. Spectrochimica Acta Part B-Atomic Spectroscopy 2008, 63 (2), 312-323.

98. Gautier, C.; Fichet, P.; Menut, D.; Lacour, J. L.; L'Hermite, D.; Dubessy, J. Study of the double-pulse setup with an orthogonal beam geometry for laser-induced breakdown spectroscopy. Spectrochimica Acta Part B-Atomic Spectroscopy 2004, 59 (7), 975-986.

99. St-Onge, L.; Sabsabi, M.; Cielo, P. Analysis of solids using laser-induced plasma spectroscopy in double-pulse mode. Spectrochimica Acta Part B-Atomic Spectroscopy 1998, 53 (3), 407-415.

100. Bogaerts, A.; Chen, Z.; Autrique, D. Double pulse laser ablation and laser induced breakdown spectroscopy: A modeling investigation. Spectrochimica Acta Part BAtomic Spectroscopy 2008, 63 (7), 746-754.

101. Scaffidi, J.; Pearman, W.; Carter, J. C.; Colston, B. W.; Angel, S. M. Temporal dependence of the enhancement of material removal in femtosecond-nanosecond dual-pulse laser-induced breakdown spectroscopy. Applied Optics 2004, 43 (35), 6492-6499.

102. Fornarini, L.; Spizzichino, V.; Colao, F.; Fantoni, R.; Lazic, V. Influence of laser wavelength on LIBS diagnostics applied to the analysis of ancient bronzes. Analytical and Bioanalytical Chemistry 2006, 385 (2), 272-280.

103. Scaffidi, J.; Angel, S. M.; Cremers, D. A. Emission enhancement mechanisms in dual-pulse LIBS. Analytical Chemistry 2006, 78 (1), 24-32.

104. St-Onge, L.; Detalle, V.; Sabsabi, M. Enhanced laser-induced breakdown spectroscopy using the combination of fourth-harmonic and fundamental $\mathrm{Nd}$ : YAG laser pulses. Spectrochimica Acta Part B-Atomic Spectroscopy 2002, 57 (1), 121-135.

105. Uebbing, J.; Brust, J.; Sdorra, W.; Leis, F.; Niemax, K. Reheating of A LaserProduced Plasma by A 2Nd Pulse Laser. Appl. Spectrosc. 1991, 45 (9), 14191423. 
106. Burakov, V.; Tarasenko, N.; Nedelko, M.; Isakov, S. Time-resolved spectroscopy and imaging diagnostics of single pulse and collinear double pulse laser induced plasma from a glass sample. Spectrochimica Acta Part B-Atomic Spectroscopy 2008, 63 (1), 19-26.

107. Cristoforetti, G.; Legnaioli, S.; Palleschi, V.; Salvetti, A.; Tognoni, E. Influence of ambient gas pressure on laser-induced breakdown spectroscopy technique in the parallel double-pulse configuration. Spectrochimica Acta Part B-Atomic Spectroscopy 2004, 59 (12), 1907-1917.

108. Pasquini, C.; Cortez, J.; Silva, L. M. C.; Gonzaga, F. B. Laser induced breakdown spectroscopy. Journal of the Brazilian Chemical Society 2007, 18 (3), 463-512.

109. Song, K.; Cha, H.; Lee, J.; Lee, Y. I. Investigation of the line-broadening mechanism for laser-induced copper plasma by time-resolved laser-induced breakdown spectroscopy. Microchemical Journal 1999, 63 (1), 53-60.

110. De Lucia, F. C.; Gottfried, J. L.; Munson, C. A.; Miziolek, A. W. Current Status of Standoff LIBS Security Applications at the United States Army Research Laboratory. Spectroscopy 2009, 24 (6), 32-38.

111. Hahn, D. W. Laser-Induced Breakdown Spectroscopy for Analysis of Aerosol Particles: The Path Toward Quantitative Analysis. Spectroscopy 2009, 24 (9), 2633.

112. Hahn, D. W.; Carranza, J. E.; Arsenault, G. R.; Johnsen, H. A.; Hencken, K. R. Aerosol generation system for development and calibration of laser-induced breakdown spectroscopy instrumentation. Review of Scientific Instruments 2001, $72(9), 3706-3713$.

113. E.M.Cahoon; J.R.Almirall Quantitative Analysis of Liquids from Aerosols and Microdrops Using Laser Induced Breakdown Spectroscopy. Analytical Chemistry 2012, DOI 10.1021/ac202834j.

114. Russo, R. E.; Mao, X. L.; Liu, H. C.; Gonzalez, J.; Mao, S. S. Laser ablation in analytical chemistry - a review. Talanta 2002, 57 (3), 425-451.

115. Jantzi, S. C.; Almirall, J. R. Characterization and forensic analysis of soil samples using laser-induced breakdown spectroscopy (LIBS). Analytical and Bioanalytical Chemistry 2011, 400 (10), 3341-3351.

116. Houk, R. S.; Fassel, V. A.; Flesch, G. D.; Svec, H. J.; Gray, A. L.; Taylor, C. E. Inductively Coupled Argon Plasma As An Ion-Source for Mass-Spectrometric Determination of Trace-Elements. Analytical Chemistry 1980, 52 (14), 22832289. 
117. Douglas, D. J.; Houk, R. S. Inductively-Coupled Plasma Mass-Spectrometry (IcpMs). Progress in Analytical Atomic Spectroscopy 1985, 8 (1), 1-18.

118. Houk, R. S.; Thompson, J. J. Inductively Coupled Plasma Mass-Spectrometry. Mass Spectrometry Reviews 1988, 7 (4), 425-461.

119. Agilent Technologies . ICP-MS Schematic. 2012. 1-15-2012.

120. D.Skoog; F.J.Holler; T.A.Nieman Principles of Instrumental Analysis; 5 ed.; Thomson Learning Inc.: 1998.

121. Mank, A. J. G.; Mason, P. R. D. A critical assessment of laser ablation ICP-MS as an analytical tool for depth analysis in silica-based glass samples. J. Anal. Atom. Spectrom. 1999, 14 (8), 1143-1153.

122. Horn, I.; Rudnick, R. L.; McDonough, W. F. Precise elemental and isotope ratio determination by simultaneous solution nebulization and laser ablation-ICP-MS: application to U-Pb geochronology. Chemical Geology 2000, 164 (3-4), 281-301.

123. Eggins, S. M.; Kinsley, L. P. J.; Shelley, J. M. G. Deposition and element fractionation processes during atmospheric pressure laser sampling for analysis by ICP-MS. Applied Surface Science 1998, 127, 278-286.

124. Houk, R. S.; Winge, R. K.; Chen, X. S. High speed photographic study of wet droplets and solid particles in the inductively coupled plasma. J. Anal. Atom. Spectrom. 1997, 12 (10), 1139-1148.

125. Aeschliman, D. B.; Bajic, S. J.; Baldwin, D. P.; Houk, R. S. High-speed digital photographic study of an inductively coupled plasma during laser ablation: comparison of dried solution aerosols from a microconcentric nebulizer and solid particles from laser ablation. J. Anal. Atom. Spectrom. 2003, 18 (9), 1008-1014.

126. Verkouteren, R. M.; Verkouteren, J. R. Inkjet Metrology: High-Accuracy Mass Measurements of Microdroplets Produced by a Drop-on-Demand Dispenser. Analytical Chemistry 2009, 81 (20), 8577-8584.

127. Zhou, J. X.; Fuh, J. Y. H.; Loh, H. T.; Wong, Y. S.; Ng, Y. S.; Gray, J. J.; Chua, S. J. Characterization of drop-on-demand microdroplet printing. International Journal of Advanced Manufacturing Technology 2010, 48 (1-4), 243-250.

128. F.Savart Memoire sur la Constitution des Veines Liquides Lancees par des Orifices Circulaires en Mince Paroi. Annales de Chimie et de Physique 1833, 53, 337.

129. L.Rayleigh On the Instability of Jets. Proc. London Math Soc. 1878, 10 (4). 
130. R.G.Sweets High frequency recording with electrostatically deflected ink-jets. Rev. Sci. Instrum. 1965, 36, 131.

131. Hansell U.S. Patent 2,512,743. 1950.

132. Sirringhaus, H.; Kawase, T.; Friend, R. H.; Shimoda, T.; Inbasekaran, M.; Wu, W.; Woo, E. P. High-resolution inkjet printing of all-polymer transistor circuits. Science 2000, 290 (5499), 2123-2126.

133. Tekin, E.; Wijlaars, H.; Holder, E.; Egbe, D. A. M.; Schubert, U. S. Film thickness dependency of the emission colors of PPE-PPVs in inkjet printed libraries. Journal of Materials Chemistry 2006, 16 (44), 4294-4298.

134. Morton, O. Solar energy: A new day dawning?: Silicon Valley sunrise. Nature 2006, 443 (7107), 19-22.

135. Contreras, M. A.; Ramanathan, K.; AbuShama, J.; Hasoon, F.; Young, D. L.; Egaas, B.; Noufi, R. Diode (characteristics in state-of-the-art $\mathrm{ZnO} / \mathrm{CdS} / \mathrm{Cu}(\mathrm{In}(1-$ x)Gax)Se-2 solar cells. Progress in Photovoltaics 2005, 13 (3), 209-216.

136. Voit, W.; Zapka, W.; Belova, L.; Rao, K. V. Application of inkjet technology for the deposition of magnetic nanoparticles to form micron-scale structures. Iee Proceedings-Science Measurement and Technology 2003, 150 (5), 252-256.

137. Crowley, K.; Morrin, A.; Shepherd, R. L.; Panhuis, M. I. H.; Wallace, G. G.; Smyth, M. R.; Killard, A. J. Fabrication of Polyaniline-Based Gas Sensors Using Piezoelectric Inkjet and Screen Printing for the Detection of Hydrogen Sulfide. Ieee Sensors Journal 2010, 10 (9), 1419-1426.

138. Hauschild, S.; Lipprandt, U.; Rumplecker, A.; Borchert, U.; Rank, A.; Schubert, R.; Forster, S. Direct preparation and loading of lipid and polymer vesicles using inkjets. Small 2005, 1 (12), 1177-1180.

139. Jayasinghe, S. N.; Qureshi, A. N.; Eagles, P. A. M. Electrohydrodynamic jet processing: An advanced electric-field-driven jetting phenomenon for processing living cells. Small 2006, 2 (2), 216-219.

140. Garcia, C. C.; Murtazin, A.; Groh, S.; Becker, M.; Niemax, K. Characterization of particles made by desolvation of monodisperse microdroplets of analyte solutions and particle suspensions for nanoparticle calibration in inductively coupled plasma spectrometry. Spectrochimica Acta Part B-Atomic Spectroscopy 2010, 65 (1), 80-85. 
141. Groh, S.; Diwakar, P. K.; Garcia, C. C.; Murtazin, A.; Hahn, D. W.; Niemax, K. 100\% Efficient Sub-Nanoliter Sample Introduction in Laser-Induced Breakdown Spectroscopy and Inductively Coupled Plasma Spectrometry: Implications for Ultralow Sample Volumes. Analytical Chemistry 2010, 82 (6), 2568-2573.

142. Le, H. P. Progress and trends in ink-jet printing technology. Journal of Imaging Science and Technology 1998, 42 (1), 49-62.

143. E.R.Lee Microdrop Generation; CRC Press: 2003.

144. Microfab Technologies . Drive Waveform Effects on Ink-Jet Device Performance. [MicroFab Technote 99-03]. 1999.

145. C.T.Crowe; D.F.Elger; J.A.Roberson; B.C.Williams Engineering Fluid Mechanics; John Wiley \& Sons, Inc.: 2009.

146. S.A.Shearer . Fluid Mechanics: Stokes' Law and Viscosity. 2012. 1-5-2012.

147. Stokes sphere. 2012. 1-12-0012.

148. Russo, R. E.; Mao, X. L.; Borisov, O. V.; Liu, H. C. Influence of wavelength on fractionation in laser ablation ICP-MS. J. Anal. Atom. Spectrom. 2000, 15 (9), 1115-1120.

149. G.A.Askar'yan Pressure on evaporation of matter in a radiation beam. Journal of experimental and theoretical physics 1963, 16, 1638.

150. Russo, R. E.; Mao, X. L.; Borisov, O. V.; Liu, H. C. Influence of wavelength on fractionation in laser ablation ICP-MS. J. Anal. Atom. Spectrom. 2000, 15 (9), 1115-1120.

151. Cabalin, L. M.; Laserna, J. J. Experimental determination of laser induced breakdown thresholds of metals under nanosecond Q-switched laser operation. Spectrochimica Acta Part B-Atomic Spectroscopy 1998, 53 (5), 723-730.

152. Mao, X. L.; Ciocan, A. C.; Borisov, O. V.; Russo, R. E. Laser ablation processes investigated using inductively coupled plasma atomic emission spectroscopy (ICP-AES). Applied Surface Science 1998, 127, 262-268.

153. Berman, L. M.; Wolf, P. J. Laser-induced breakdown spectroscopy of liquids: Aqueous solutions of nickel and chlorinated hydrocarbons. Appl. Spectrosc. 1998, $52(3), 438-443$.

154. T.Ishizuka . Laser emission spectrography of rare earth elements. Analytical Chemistry 45, 538. 1973.

155. C.Girault Université de Limoges, 1990. 
156. Geertsen, C.; Briand, A.; Chartier, F.; Lacour, J. L.; Mauchien, P.; Sjostrom, S.; Mermet, J. M. Comparison Between Infrared and Ultraviolet-Laser Ablation at Atmospheric-Pressure - Implications for Solid Sampling Inductively-Coupled Plasma Spectrometry. J. Anal. Atom. Spectrom. 1994, 9 (1), 17-22.

157. Santagata, A.; De Bonis, A.; Villani, P.; Teghil, R.; Parisi, G. P. Fs/ns-dual-pulsel orthogonal geometry plasma plume reheating for copper-based-alloys analysis. Applied Surface Science 2006, 252 (13), 4685-4690.

158. De Lucia, F. C.; Gottfried, J. L.; Munson, C. A.; Miziolek, A. W. Double pulse laser-induced breakdown spectroscopy of explosives: Initial study towards improved discrimination. Spectrochimica Acta Part B-Atomic Spectroscopy 2007, 62 (12), 1399-1404.

159. Stratis, D. N.; Eland, K. L.; Angel, S. M. Enhancement of aluminum, titanium, and iron in glass using pre-ablation spark dual-pulse LIBS. Appl. Spectrosc. 2000, 54 (12), 1719-1726.

160. Scaffidi, J.; Pender, J.; Pearman, W.; Goode, S. R.; Colston, B. W.; Carter, J. C.; Angel, S. M. Dual-pulse laser-induced breakdown spectroscopy with combinations of femtosecond and nanosecond laser pulses. Applied Optics 2003, 42 (30), 6099-6106.

161. Cristoforetti, G.; Lorenzetti, G.; Legnaioli, S.; Palleschi, V. Investigation on the role of air in the dynamical evolution and thermodynamic state of a laser-induced aluminium plasma by spatial- and time-resolved spectroscopy. Spectrochimica Acta Part B-Atomic Spectroscopy 2010, 65 (9-10), 787-796.

162. Kurniawan, H.; Nakajima, S.; Batubara, J. E.; Marpaung, M.; Okamoto, M. LaserInduced Shock-Wave Plasma in Glass and Its Application to Elemental Analysis. Appl. Spectrosc. 1995, 49 (8), 1067-1072.

163. Ismail, M. A.; Cristoforetti, G.; Legnaioli, S.; Pardini, L.; Palleschi, V.; Salvetti, A.; Tognoni, E.; Harith, M. A. Comparison of detection limits, for two metallic matrices, of laser-induced breakdown spectroscopy in the single and double-pulse configurations. Analytical and Bioanalytical Chemistry 2006, 385 (2), 316-325.

164. Yamamoto, K. Y.; Cremers, D. A.; Ferris, M. J.; Foster, L. E. Detection of metals in the environment using a portable laser-induced breakdown spectroscopy instrument. Appl. Spectrosc. 1996, 50 (2), 222-233.

165. David A.Cremers; J.E.Barefield II; A.C.Koskelo . Remote elemental analysis by laser-induced breakdown spectroscopy using a fiber-optic cable. Applied Spectroscopy 49, 857. 1995. 
166. T.Trejos; J.Almirall Elemental Analysis of Glass; Second Round Robin Report; 09.

167. R.Koons; T.Trejos; J.Almirall Elemental Analysis Working Group NIJ Award 2009-DN-BX-K252, Third Round Robin; 10.

168. R.Koons; T.Trejos; J.Almirall Elemental Analysis Working Group NIJ Award 2009-DN-BX-K252 Fourth Round Robin; 11.

169. Bridge, C. M.; Powell, J.; Steele, K. L.; Williams, M.; MacInnis, J. M.; Sigman, M. E. Characterization of automobile float glass with laser-induced breakdown spectroscopy and laser ablation inductively coupled plasma mass spectrometry. Applied Spectroscopy 2006, 60 (10), 1181-1187.

170. Naes, B. E.; Umpierrez, S.; Ryland, S.; Barnett, C.; Almirall, J. R. A comparison of laser ablation inductively coupled plasma mass spectrometry, micro X-ray fluorescence spectroscopy, and laser induced breakdown spectroscopy for the discrimination of automotive glass. Spectrochimica Acta Part B-Atomic Spectroscopy 2008, 63 (10), 1145-1150.

171. Ito, M.; Hokura, A.; Oishi, M.; Nishiwaki, Y.; Nakai, I. Characterization of sheet glass based on trace elements analysis using LA-ICP-MS and their applications to forensic science. Bunseki Kagaku 2007, 56 (12), 1115-1125.

172. Berends-Montero, S.; Wiarda, W.; de Joode, P.; van der Peijl, G. Forensic analysis of float glass using laser ablation inductively coupled plasma mass spectrometry (LA-ICP-MS): validation of a method. Journal of Analytical Atomic Spectrometry 2006, 21 (11), 1185-1193.

173. Hordijk, M.; Wiarda, W.; de Joode, P.; van der Peijl, G.; Montero, S. LA-ICP-MS method for the elemental profiling of forensic glass: A study of a float glass sample set collected in the Netherlands. Forensic Science International 2003, 136, 358-359.

174. Smith, K.; Trejos, T.; Watling, R. J.; Almirall, J. A guide for the quantitative elemental analysis of glass using laser ablation inductively coupled plasma mass spectrometry. Atomic Spectroscopy 2006, 27 (3), 69-75.

175. Fisher, B. T.; Johnsen, H. A.; Buckley, S. G.; Hahn, D. W. Temporal gating for the optimization of laser-induced breakdown spectroscopy detection and analysis of toxic metals. Applied Spectroscopy 2001, 55 (10), 1312-1319.

176. Wainner, R. T.; Harmon, R. S.; Miziolek, A. W.; McNesby, K. L.; French, P. D. Analysis of environmental lead contamination: comparison of LIBS field and laboratory instruments. Spectrochimica Acta Part B-Atomic Spectroscopy 2001, 56 (6), 777-793. 
177. Gautier, C.; Fichet, P.; Menut, D.; Lacour, J. L.; L'Hermite, D.; Dubessy, J. Quantification of the intensity enhancements for the double-pulse laser-induced breakdown spectroscopy in the orthogonal beam geometry. Spectrochimica Acta Part B-Atomic Spectroscopy 2005, 60 (2), 265-276.

178. St-Onge, L.; Detalle, V.; Sabsabi, M. Enhanced laser-induced breakdown spectroscopy using the combination of fourth-harmonic and fundamental Nd : YAG laser pulses. Spectrochimica Acta Part B-Atomic Spectroscopy 2002, 57 (1), 121-135.

179. Milan, M.; Vadillo, J. M.; Laserna, J. J. Removal of air interference in laserinduced breakdown spectrometry monitored by spatially and temporally resolved charge-coupled device measurements. Journal of Analytical Atomic Spectrometry 1997, 12 (4), 441-444.

180. Iida, Y. Effects of Atmosphere on Laser Vaporization and Excitation Processes of Solid Samples. Spectrochimica Acta Part B-Atomic Spectroscopy 1990, 45 (12), 1353-1367.

181. Wisbrun, R.; Schechter, I.; Niessner, R.; Schroder, H.; Kompa, K. L. Detector for Trace Elemental Analysis of Solid Environmental-Samples by Laser-Plasma Spectroscopy. Analytical Chemistry 1994, 66 (18), 2964-2975.

182. Aguilera, J. A.; Aragon, C. Characterization of a laser-induced plasma by spatially resolved spectroscopy of neutral atom and ion emissions. Comparison of local and spatially integrated measurements. Spectrochimica Acta Part B-Atomic Spectroscopy 2004, 59 (12), 1861-1876.

183. Hakkanen, H.; Houni, J.; Kaski, S.; Korppi-Tommola, J. E. I. Analysis of paper by laser-induced plasma spectroscopy. Spectrochimica Acta Part B-Atomic Spectroscopy 2001, 56 (6), 737-742.

184. Vadillo, J. M.; Palanco, S.; Romero, M. D.; Laserna, J. J. Applications of laserinduced breakdown spectrometry (LIBS) in surface analysis. Fresenius Journal of Analytical Chemistry 1996, 355 (7-8), 909-912.

185. Menut, D.; Fichet, P.; Lacour, J. L.; Rivoallan, A.; Mauchien, P. Micro-laserinduced breakdown spectroscopy technique: a powerful method for performing quantitative surface mapping on conductive and nonconductive samples. Applied Optics 2003, 42 (30), 6063-6071.

186. Ko, J. B.; Sdorra, W.; Niemax, K. On the Internal Standardization in OpticalEmission Spectrometry of Microplasmas Produced by Laser Ablation of Solid Samples. Fresenius Zeitschrift fur Analytische Chemie 1989, 335 (7), 648-651. 
187. Fichet, P.; Tabarant, M.; Salle, B.; Gautier, C. Comparisons between LIBS and ICP/OES. Analytical and Bioanalytical Chemistry 2006, 385 (2), 338-344.

188. St-Onge, L.; Kwong, E.; Sabsabi, M.; Vadas, E. B. Quantitative analysis of pharmaceutical products by laser-induced breakdown spectroscopy. Spectrochimica Acta Part B-Atomic Spectroscopy 2002, 57 (7), 1131-1140.

189. De Lucia, F. C.; Gottfried, J. L. Classification of explosive residues on organic substrates using laser induced breakdown spectroscopy. Applied Optics 2012, 51 (7), B83-B92.

190. Lazic, V.; Palucci, A.; Jovicevic, S.; Poggi, C.; Buono, E. Analysis of explosive and other organic residues by laser induced breakdown spectroscopy. Spectrochimica Acta Part B-Atomic Spectroscopy 2009, 64 (10), 1028-1039.

191. Trejos, T.; Flores, A.; Almirall, J. R. Micro-spectrochemical analysis of document paper and gel inks by laser ablation inductively coupled plasma mass spectrometry and laser induced breakdown spectroscopy. Spectrochimica Acta Part B-Atomic Spectroscopy 2010, 65 (11), 884-895.

192. Gondal, M. A.; Nasr, M. M.; Ahmed, M. M.; Yamani, Z. H.; Alsalhi, M. S. Detection of lead in paint samples synthesized locally using-laser-induced breakdown spectroscopy. Journal of Environmental Science and Health Part AToxic/Hazardous Substances \& Environmental Engineering 2011, 46 (1), 42-49.

193. McIntee, E.; Viglino, E.; Rinke, C.; Kumor, S.; Ni, L. Q.; Sigman, M. E. Comparative analysis of automotive paints by laser induced breakdown spectroscopy and nonparametric permutation tests. Spectrochimica Acta Part BAtomic Spectroscopy 2010, 65 (7), 542-548. 
April 8, 1977

2006

2009-2012

2010

2010

2011

2011

2012

Born, Bismarck ND, USA

B.S. in Chemistry, Minor in Business, Florida International University Teaching and Research Assistant $2^{\text {nd }}$ Place Poster Award, $6^{\text {th }}$ International LIBS Conference $3^{\text {rd }}$ Place Oral Presenter, Scholarly Forum, Florida International University $1^{\text {st }}$ Place Oral Presenter, Scholarly Forum, Florida International University Dissertation Year Fellowship Award, Florida International University $1^{\text {st }}$ Place Oral Presenter, Scholarly Forum, Florida International University 2012 Outstanding Graduate Student (PhD in Chemistry), Florida International University PUBLICATIONS

E. Cahoon, J. Almirall; Anal. Chem., Jan. 2012, DOI 10.1021/ac202834j

E. Cahoon, J. Almirall; Applied Optics, 49 (13), 2010

C. Barnett, E. Cahoon, J. Almirall; Spectrochim. Acta, Part B 1016 (63), 2008

E. Cahoon, J. Almirall; Analytical Laser Induced Breakdown Spectroscopy for Discrimination of Glass Samples from the Same Manufacturing Plants, submitted

E. Cahoon, J. Almirall; The association and discrimination of float glass by LA-ICP-MS from single manufacturing plants, submitted

\section{PRESENTATIONS}

Annual International Forensic Research Institute (IFRI) Symposium, March 9-10 2012, Florida International University, Miami, FL

Graduate Student Association Scholarly Forum: Oral presentation, February 27, 2012, Florida International University, Miami, FL 
Lawrence Berkeley National Laboratory: Invited seminar speaker, February 1, 2012, Berkeley, CA

The Federation of Analytical Chemistry and Spectroscopy (FACSS): Invited speaker, presentation titled, October 2-7, 2011, Reno, NV

National Institute of Justice 2011 Trace Evidence Symposium: Science, Significance and Impact: Poster presentation, August 8-11, 2011, Kansas City, MO

North American Symposium on Laser Induced Breakdown Spectroscopy (NASLIBS) 2011: Oral presentation, July 18-20, 2011, Clearwater Beach, FL

Graduate Student Association Scholarly Forum: Oral presentation, March 31, 2011, Florida International University, Miami, FL

The Pittsburgh Conference on Analytical Chemistry and Applied Spectroscopy (PITTCON) Meeting 2011: Oral presentation, March 13-18, 2011, Atlanta, GA

NIJ Sponsored Elemental Analysis Working Group Meeting 2010: Oral presentation, December 1-3, 2010, Florida International University, Miami, FL

6th International Conference of Laser-Induced Breakdown Spectroscopy 2010: Poster presentation, September 13-17, 2010, Memphis, TN

NIJ Sponsored Elemental Analysis Working Group Meeting 2010: Oral presentation, Aug. 13, 2010, Breckenridge, CO

American Chemical Society FAME (Florida Annual Meeting and Exposition) 2010: Oral presentation, May 13-15, 2010, Palm Harbor, FL

Graduate Student Association Scholarly Forum: Oral presentation, March 22, 2010, Florida International University, Miami, FL

American Academy of Forensic Sciences 2010: Oral presentation, February, Seattle, WA North American Symposium on Laser Induced Breakdown Spectroscopy (NASLIBS) 2009: Poster presentation, July 13-15, 2009, New Orleans, LA

American Chemical Society FAME (Florida Annual Meeting and Exposition) 2009: Oral presentation, Orlando, FL

American Academy of Forensic Sciences 2008: Poster presentation, February, San Antonio, TX 